\title{
The VLT-FLAMES survey of massive stars: constraints on stellar evolution from the chemical compositions of rapidly rotating Galactic and Magellanic Cloud B-type stars ${ }^{\star}, \star \star$
}

\author{
I. Hunter ${ }^{1}$, I. Brott ${ }^{2}$, N. Langer ${ }^{2}$, D. J. Lennon ${ }^{3}$, P. L. Dufton ${ }^{1}$, I. D. Howarth ${ }^{4}$, R. S. I. Ryans ${ }^{1}$, \\ C. Trundle ${ }^{1}$, C. J. Evans ${ }^{5}$, A. de Koter ${ }^{6,2}$, and S. J. Smartt ${ }^{1}$ \\ 1 Astrophysics Research Centre, School of Mathematics \& Physics, The Queen's University of Belfast, Belfast, BT7 1NN, \\ Northern Ireland, UK \\ e-mail: i.hunter@qub.ac.uk \\ Astronomical Institute, Utrecht University, Princetonplein 5, 3584CC, Utrecht, The Netherlands \\ Space Telescope Science Institute, 3700 San Martin Drive, Baltimore, MD 21218, USA \\ 4 Department of Physics and Astronomy, University College London, Gower Street, London WC1E 6BT, UK \\ 5 UK Astronomy Technology Centre, Royal Observatory, Edinburgh, Blackford Hill, Edinburgh, EH9 3HJ, UK \\ 6 Astronomical Institute Anton Pannekoek, University of Amsterdam, Kruislaan 403, 1098 SJ Amsterdam, The Netherlands
}

Received 7 April 2008 / Accepted 5 January 2009

\section{ABSTRACT}

\begin{abstract}
Aims. We have previously analysed the spectra of 135 early B-type stars in the Large Magellanic Cloud (LMC) and found several groups of stars that have chemical compositions that conflict with the theory of rotational mixing. Here we extend this study to Galactic and Small Magellanic Cloud (SMC) metallicities.

Methods. We provide chemical compositions for $\sim 50$ Galactic and $\sim 100$ SMC early B-type stars and compare these to the LMC results. These samples cover a range of projected rotational velocities up to $\sim 300 \mathrm{~km} \mathrm{~s}^{-1}$ and hence are well suited to testing rotational mixing models. The surface nitrogen abundances are utilised as a probe of the mixing process since nitrogen is synthesized in the core of the stars and mixed to the surface.

Results. In the SMC, we find a population of slowly rotating nitrogen-rich stars amongst the early B type core-hydrogen burning stars, which is comparable to that found previously in the LMC. The identification of non-enriched rapid rotators in the SMC is not possible due to the relatively high upper limits on the nitrogen abundance for the fast rotators. In the Galactic sample we find no significant enrichment amongst the core hydrogen-burning stars, which appears to be in contrast with the expectation from both rotating single-star and close binary evolution models. However, only a small number of the rapidly rotating stars have evolved enough to produce a significant nitrogen enrichment, and these may be analogous to the non-enriched rapid rotators previously found in the LMC sample. Finally, in each metallicity regime, a population of highly enriched supergiants is observed, which cannot be the immediate descendants of core-hydrogen burning stars. Their abundances are, however, compatible with them having gone through a previous red supergiant phase. Together, these observations paint a complex picture of the nitrogen enrichment in massive main sequence and supergiant stellar atmospheres, where age and binarity cause crucial effects. Whether rotational mixing is required to understand our results remains an open question at this time, but could be answered by identifying the true binary fraction in those groups of stars that do not agree with single-star evolutionary models.
\end{abstract}

Key words. stars: early-type - stars: atmospheres - stars: rotation - stars: abundances - stars: evolution - galaxies: Magellanic Clouds

\section{Introduction}

Rotation is generally considered to be of critical importance for theoretical models of massive star evolution and, specifically, rotationally-induced mixing, where material is mixed from the stellar core into the photosphere (Heger \& Langer 2000; Meynet $\&$ Maeder 2000). For example, rotation has been used as an explanation of blue to red supergiant ratios (Maeder \& Meynet 2001) and of Wolf-Rayet populations as a function of metallicity (Meynet \& Maeder 2005; Vink \& de Koter 2005). Additionally, for a single massive star to end its life with an associated long gamma-ray burst, chemically homogeneous evolution through

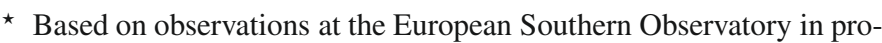
grammes 171.0237 and 073.0234 .

$\star \star$ Figure 1 and Tables 1 and 2 are only available in electronic form at http://www . aanda.org
}

rapid rotation may be required (Yoon \& Langer 2005; Woosley $\&$ Heger 2006)

Stellar evolution models including rotation predict a surface enrichment of helium and nitrogen with an associated carbon and oxygen depletion during the main sequence evolution. Massive star surface abundance anomalies (particularly nitrogen) have long been observed, by, for example, Walborn (1970), Dufton (1972) and, more recently, Gies \& Lambert (1992), Kilian (1991), Bouret et al. (2003), Dufton et al. (2005), Lennon et al. (2003), Trundle \& Lennon (2005), Korn et al. (2002) and Venn (1999). However, the majority of these analyses have focused on one of two groups: narrow lined main-sequence stars (i.e. stars with low projected rotational velocities) or blue supergiants. For example, Kilian considered 21 main sequence Galactic objects all with projected rotational velocities of less than $63 \mathrm{~km} \mathrm{~s}^{-1}$. 
The magnitude of the predicted mixing has generally been compared to and calibrated against these two groups. It follows from the stellar evolution models that the more rapidly a star rotates, the more mixing will occur, and hence the greater the nitrogen (and helium) surface enrichment that should be observed. Unfortunately spectroscopic studies of rapidly rotating stars are relatively rare. Lennon et al. (1991) studied the O9.5V star HD 93521 with a projected rotational velocity of approximately $400 \mathrm{~km} \mathrm{~s}^{-1}$. They deduced an enhanced helium abundance that they ascribed to rotational mixing. Howarth \& Smith (2001) analysed spectra of three rapidly rotating O-type stars (including HD 93 521) using models that allowed for variations in temperature and gravity across the stellar surface and again found evidence for helium enhancements. By contrast, Villamariz and Herrero (2005) obtained a normal helium abundance together with a nitrogen enrichment for one of the stars ( $\zeta$ Oph) discussed by Howarth and Smith.

Vrancken et al. (1997) analysed the spectra of two B-type targets in NGC 2244, with projected rotational velocities between 200 and $300 \mathrm{~km} \mathrm{~s}^{-1}$. The analysis was undertaken differentially with respect to a cluster member with a small projected rotational velocity. No significant abundance anomalies were identified with indeed the two fast rotators being found to have a small underabundance of nitrogen. Daflon et al. (2001) analysed twelve Galactic stars in Cygnus associations with projected rotational velocities between 60 and $150 \mathrm{~km} \mathrm{~s}^{-1}$. On average they had subsolar abundances that agreed with those found for a sample of eight stars with very low projected rotational velocities. However two targets (with projected rotational velocities of 100 and $142 \mathrm{~km} \mathrm{~s}^{-1}$ ) showed relative nitrogen enhancements of nearly a factor of two. Korn et al. (2005) analysed three LMC targets with projected rotational velocities of approximately $130 \mathrm{~km} \mathrm{~s}^{-1}$ but again found no evidence for either enhanced helium or nitrogen abundances.

The measurement of surface abundances of a large sample of core-hydrogen burning, rapidly rotating, massive stars is clearly necessary for both testing and calibrating the mixing theory and this was one of the primary drivers of the VLT-FLAMES survey of massive stars. This Large Program on the VLT with the FLAMES instrument (PI.: S.J. Smartt) focused on OB-type stars in the Galaxy and Magellanic Clouds (Evans et al. 2005; Evans et al. 2006, hereafter Papers I and II). Over 700 O- and B-type stars were observed across these three metallicity regimes. Both the large number of objects and the different metallicity regimes allow many of the theoretical predictions of evolutionary models to be tested.

The principle outcomes of this survey related to rotation are as follows. Mokiem et al. (2006, 2007) have analysed the O-type stars in the sample ( $\sim 50$ objects) and used their massloss rates to derive the wind-momentum luminosity relation. They show that at lower metallicity stars rotate faster since they have lower mass loss rates. Dufton et al. (2006, hereafter Paper III) have shown that Galactic cluster stars (observed in the survey) rotate significantly faster than stars in the Galactic field. This was consistent with studies of stars in the double cluster h and $\chi$ Persei (Slettebak 1968; Strom et al. 2005) and other clusters (Wolff et al. 2007; Huang and Gies 2006a). Hunter et al. (2008a, hereafter Paper IV) have derived rotational velocities for the Magellanic Cloud B-type stars from the survey and show that stars at low metallicity rotate faster than in higher metallicity regimes. For two clusters (NGC 330 and NGC 2004), these results complement the recent studies of Martayan et al. (2006, 2007), who also utilised the FLAMES spectrograph albeit at lower spectral resolution. Additionally in Paper IV, it was suggested that the observed population of B-type supergiants cannot be explained by normal single star evolution and either binarity or blue-loops needed to be invoked to model the population.

Hunter et al. (2007, hereafter Paper V) have performed a detailed chemical composition analysis of approximately 50 narrow-lined B-type stars from the survey and utilised these objects to estimate the baseline chemical compositions of the Magellanic Clouds, thereby complementing previous studies mainly of H II regions (summarized, for example, by Garnett 1999). Additionally as found from H II region analyses and other more limited stellar samples (for example, Korn et al. 2002), they confirmed that for both Magellanic Clouds the assumption that all elements can be scaled from the solar composition by the same factor is incorrect. Trundle et al. (2007; hereafter Paper VI) have extended this sample of narrow lined stars to $\sim 100$ objects and, utilising the same methods, have derived temperature scales for Galactic and Magellanic Cloud stars, which imply that stars at lower metallicity have higher effective temperatures for a given spectral type, in broad agreement with previous studies (Martins et al. 2002, 2005; Crowther et al. 2002, 2006; Massey et al. 2004, 2005). Hunter et al. (2008b, hereafter Paper VII) have presented chemical compositions for $135 \mathrm{~B}$-type stars in the Large Magellanic Cloud (LMC) with a broad range of rotational velocities (up to $\sim 350 \mathrm{~km} \mathrm{~s}^{-1}$ ). This was the first significant abundance analysis of rapidly rotating early B-type stars and they utilised the nitrogen abundances to test the theory of rotational mixing, finding the theory to be unable to explain several aspects of the sample. In particular they found populations of unenriched fast rotators, highly enriched slow rotators and supergiants that are highly enriched compared to normal corehydrogen burning objects (see Sect. 4). In this paper we utilise identical methodologies to Paper VII and extend the chemical composition analysis to the Galactic and Small Magellanic Cloud (SMC) samples from the FLAMES large survey and compare these to the LMC stars.

In Sect. 2 we briefly describe the survey and the selection criteria for the objects that are analysed here. In Sect. 3 a summary of the analysis methodology is presented and the chemical compositions of the objects in each metallicity regime are compared and discussed. In Sect. 4 the nitrogen abundances of the sample are used to test the validity of the conclusions made in Paper VII and to further constrain the theory of rotational mixing. Finally in Sect. 5 we present our principle findings and lay out the challenges for future theoretical models.

\section{Observations}

The observations of the Galactic and Magellanic Cloud stars from the VLT-FLAMES survey of massive stars have been described in detail in Paper I and Paper II respectively. To summarise, the majority of the data were obtained using the Fibre Large Array Multi-Element Spectrograph (FLAMES) on the $8.2 \mathrm{~m}$ European Southern Observatory Very Large Telescope (ESO-VLT) at Paranal, Chile. These data were supplemented by UVES and FEROS observations of the brighter targets. Approximately 300 Galactic objects were observed and these are associated with the clusters NGC 6611, NGC 3293 and NGC 4755. Due to the distance of the Magellanic Clouds and constraints on fibre placement with the FLAMES instrument, it was not possible to solely observe cluster objects and hence the observed Magellanic Cloud samples are dominated by the field populations (see Paper IV). These field samples were centred towards N 11 and NGC 2004 in the LMC and NGC 346 and 
NGC 330 in the SMC, with over 400 objects being observed. The signal-to-noise $(S / N)$ ratios of the SMC spectra were generally in the range $25-150$ while the other spectra were in the range $50-200^{1}$.

In Fig. 1 (only available online) we provide the observed spectra for a subset of the LMC stars. The strongest nitrogen line (at $3995 \AA$ ) has been highlighted and the $\mathrm{H} \alpha$ profile is also shown where available. Panels (a) to (d), (e) to (h) and (i) to (l) show objects with low projected rotational velocities (less than $100 \mathrm{~km} \mathrm{~s}^{-1}$ ), high projected rotational velocities (greater than $200 \mathrm{~km} \mathrm{~s}^{-1}$ ) and supergiants respectively. In each of these groups two relatively nitrogen normal and two nitrogen rich stars are displayed. Inspection of the spectra reveals that apart from the nitrogen lines there is no apparent difference between the spectra of nitrogen rich and nitrogen normal stars.

\subsection{Selection criteria}

Paper IV presents atmospheric parameters and projected rotational velocities for the SMC sample of stars from the FLAMES survey. We have examined the B-type stars in this sample of objects and estimated chemical compositions where possible. Following the methodology adopted for the LMC sample, upper limits to the nitrogen abundances have been estimated when no nitrogen lines were observed for the SMC objects. Stars were excluded from the analysis if asymmetries in the line were obvious or if the upper limits to the equivalent width estimates for the nitrogen lines lead to abundance estimates that did not provide a useful constraint. Note that due to spectral contamination issues Paper IV does not provide atmospheric parameters for the double-lined spectroscopic systems or the Be-type stars and hence no attempt has been made to derive abundances for these objects here. Additionally, due to limitations in the model atmosphere grid used to derive the abundances, the majority of the O-type stars (i.e. stars hotter than $35000 \mathrm{~K}$ ) have not been analysed (see Sect. 3). These selection criteria are consistent with those for the LMC sample (Paper VII). We note that the two B8 supergiants analysed in Paper VI, NGC 2004-005 and NGC 2004-007, were excluded as their oxygen abundances may be unreliable.

In addition to the Magellanic Cloud samples, we require a Galactic comparison sample. However, given that we are primarily interested in using nitrogen to constrain the possible enrichment process (see Sect. 1), chemical compositions are only presented for those Galactic stars with atmospheric parameters in Paper III and measurable nitrogen features. Although this introduces a bias towards slow rotators in the Galactic sample, the upper limits to the nitrogen abundances for the faster rotators were too high to be useful when comparing with evolutionary models. For the Galactic sample nitrogen lines were observable at projected rotational velocities up to $\sim 250 \mathrm{~km} \mathrm{~s}^{-1}$. In Fig. 2 the projected rotational velocity distributions of the selected stars in the three regions are plotted. Despite the selection criteria, the three distributions are similar although we observe a smaller proportion of slowly rotating Galactic objects. This may be due to Galactic cluster stars rotating faster than field stars (see Strom et al. 2005; Paper III; Huang \& Gies 2006a; Wolff et al. 2007). Hence our Galactic and Magellanic Cloud samples can be considered to be comparable, with our selection criteria compensating for the intrinsic differences between the Galactic cluster and the Magellanic Cloud field star velocity distributions.

1 The reduced spectra are publicly available at http://star.pst. qub.ac.uk/ sjs/flames/

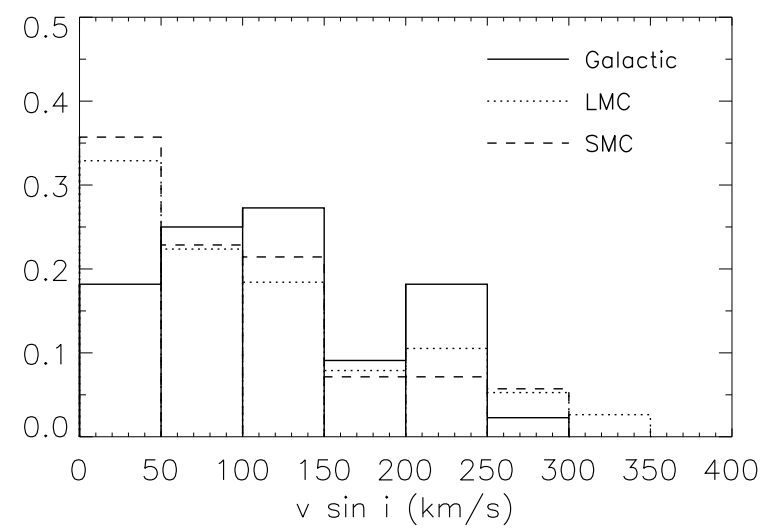

Fig. 2. Histogram of the projected rotational velocity distribution of the Galactic, LMC and SMC samples, normalised to the number of stars in the sample. The three samples have similar distributions save for a slight deficit of slow rotators in the Galactic sample.

Papers V and VI have presented the abundances for the majority of the slow rotators in these samples and these abundances have been adopted here. Those stars which have previously been identified as radial velocity variables (Papers I-IV) are considered to be binaries in the subsequent discussion.

\subsection{Equivalent width measurements}

As discussed in Papers V and VI the equivalent widths ( $E W s)$ of the absorption lines for the narrow lined stars were measured by fitting Gaussian profiles to the observed features. The uncertainties in these measurements are typically estimated to be of the order of $10 \%$ for well defined lines (Paper V). However, for faster rotating stars (typically those with projected rotational velocities greater than $50 \mathrm{~km} \mathrm{~s}^{-1}$ ) the rotational broadening dominates over the other broadening mechanisms and the use of a Gaussian profile is no longer satisfactory. Hence we have utilised rotationally broadened profiles to estimate the equivalent widths of the faster rotators in our sample.

The measurement of the metal line equivalent widths for the fast rotators is not as straightforward as the measurements for the slowly rotating stars analysed in Papers V and VI and many of the associated problems and uncertainties are discussed in Vrancken et al. (1997). For example, at high rotational velocities part of the line can be "hidden" in the continuum. This obviously will affect the estimation of the continuum level, the line strength and the line width and can bias the measurements towards lower equivalent widths. However, a priori knowledge of the projected rotational velocity can help to alleviate these problems. The rotational velocity of the fast rotators have been estimated from the helium lines (Papers III and IV) where these problems are less important due to the strength of these lines. In the estimation of the equivalent widths of the metal lines we have forced the fits to the lines to have a width equivalent to the projected rotational velocity, and hence the line width is not underestimated. Additionally, in defining the continuum level the continuum is set beyond the region of the broad lines and is fitted with a low order polynomial.

In Fig. 3 examples are shown to demonstrate the accuracy of the equivalent width measurements in fast rotating stars for lines of different strength and spectra of differing quality. A theoretical line profile of a known equivalent width has been rotationally broadened and random noise is added to the spectra. This line is then fitted with a rotationally broadened profile. The 


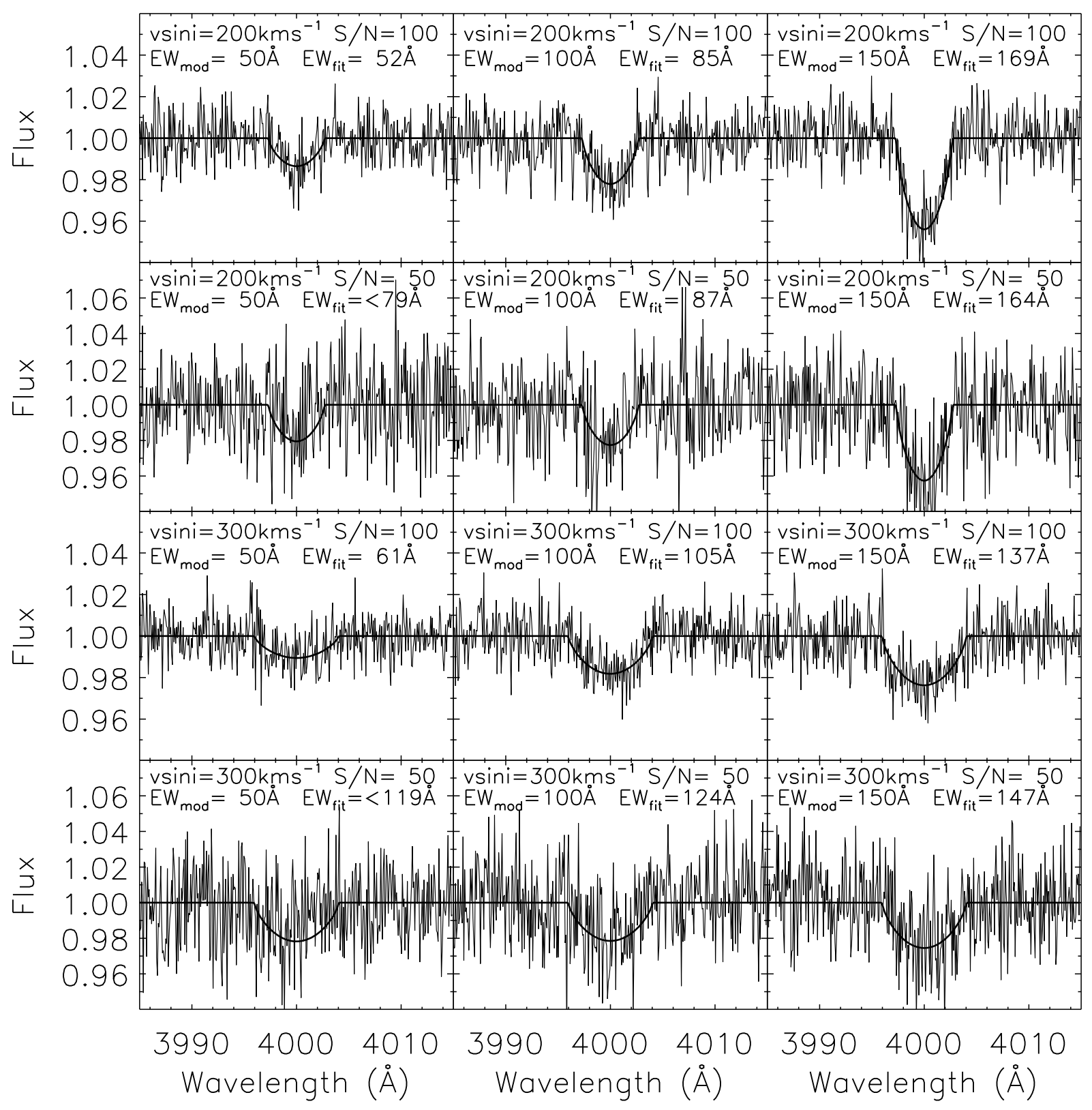

Fig. 3. Examples of the quality of the equivalent width measurements for fast rotators at differing $S / N$ ratios. The theoretical spectra of an absorption line (with an equivalent width of $E W_{\bmod }$ ) is rotationally broadened and noise added to the spectra. Using our IDL fitting routine the equivalent width of this simulated line is then measured ( $\left.E W_{\text {fit }}\right)$. It should be noted that at a $S / N$ ratio of 50 , an absorption line of $50 \mathrm{~m} \AA$ could not be observed at these rotational velocities and therefore upper limits to the equivalent width are given.

centroid and width of the line are known quantities and hence these parameters can be constrained. We can therefore estimate the uncertainty in the equivalent width measurements, excluding continuum fitting, to be of the order of $\sim 20 \%$. Although we have carefully defined the continuum region to be beyond the region of the line, we estimate, by performing repeated fits to a subsample of spectra, that the normalisation procedure contributes an additional error of $\sim 20 \%$ to the equivalent width measurements. Hence for the faster rotators in our sample the equivalent width errors can be considered to be of the order of $20-40 \%$.

The blending of lines in rotationally broadened spectra also affects the equivalent width measurements. We again define the continuum beyond the range of the blended lines and fit the blended lines simultaneously using multiple rotationally broadened profiles. By constraining the fitted lines to have the appropriate centroids, rotational broadening errors in the fitting procedure can be minimised. If this procedure does not result in a visually satisfactory fit the lines have not been included for further analysis. However, weak absorption features which are not included will obviously affect the measured equivalent widths especially for the faster rotators and would bias the measurements to higher equivalent widths. Comparison of the abundances between the slow rotators (where blending is not an issue) with the faster rotators reveals no trend with rotational velocity. Hence we can conclude that while weak absorption lines may bias our equivalent width measurements, such a bias is negligible within the other uncertainties in our analysis.

The absorption lines used in this analysis are summarized in Paper V with only measurable $\mathrm{C}, \mathrm{O}, \mathrm{Mg}$ and $\mathrm{Si}$ lines being included. However, given that we are primarily interested in the nitrogen abundances, even upper limits to the nitrogen abundance of a star can be useful for constraining the chemical mixing 
processes in the Magellanic Cloud data sets. Hence, as discussed in Sect. 2.1 for the Magellanic Cloud samples, upper limits to the equivalent width of the N II $3995 \AA$ line (which is the strongest nitrogen feature in our spectra) and to the corresponding nitrogen abundance were estimated. Examples of the fitting of upper limits are also shown in Fig. 3. The equivalent widths of the lines included in this analysis along with the estimated abundances (see Sect. 3.2) are given in Table 1 (only available online).

\section{Analysis}

\subsection{Atmospheric parameters and rotational velocities}

The atmospheric parameters and rotational velocities have been estimated in Papers III and IV and for completeness a brief overview of the method is given here. The non-LTE TLUSTY model atmosphere grid (see Hubeny \& Lanz 1995, and references therein) has been used and is described in detail in Dufton et al. $(2005)^{2}$. This grid covers the effective temperature range $12000-35000 \mathrm{~K}$ and allows for the analysis of all the B-type stars observed in the VLT-FLAMES survey, both the core-hydrogen burning and supergiant objects.

As described in Papers III and IV the hydrogen and helium lines were utilised to estimate the effective temperatures and surface gravities of the majority of the sample. For those stars where the helium lines were not temperature sensitive, effective temperatures were estimated based on spectral type using the effective temperature calibrations from Paper VI. The hydrogen lines were used to constrain the surface gravities in all cases.

For the narrow-lined spectra, where two ionization stages of silicon could be observed, the effective temperature was determined from the silicon ionization balance. The majority of these stars have been analysed in Papers V and VI. For those stars in which two ionization stages were available, but which lay outside the selection criteria of these papers, the effective temperatures (and if necessary the surface gravities) have been re-determined, rather than using those estimated in Papers III and IV. However these parameters are in good agreement with those previously determined.

Rotational velocities have generally been estimated from the profile fitting methodology, where a theoretical spectrum is rotationally broadened to fit the observed spectrum. Again these values are given in Papers III and IV. We note that for several cases it was found that the rotational velocity estimated from the helium lines (Papers III and IV) did not well fit the metal lines and in these few cases the projected rotational velocity has been re-determined from the metal lines and is preferred. For the supergiants in the sample, where macroturbulent broadening can dominate over rotational broadening, the Fourier Transform technique has been utilised. This technique has the advantage that rotational broadening can be separated from other broadening mechanisms (see Simón-Díaz \& Herrero 2007 and Paper IV).

\subsection{Abundance determinations}

Carbon, nitrogen, oxygen, magnesium and silicon abundances have been estimated using the atmospheric parameters and $E W$ measurements. In order to constrain the microturbulence, it has been assumed that the silicon abundance should be invariant across each metallicity regime and the microturbulent velocity has been fixed to achieve this where possible. A detailed description of this methodology is given in Paper V. The chemical

\footnotetext{
${ }^{2}$ See also http://star.pst.qub.ac.uk
}

composition of each star is given in Table 2 (only available online). This table lists the stellar identifier (from Papers I and II), atmospheric parameters (effective temperature, surface gravity and microturbulence), projected rotational velocity and chemical composition ( $\mathrm{C}, \mathrm{N}, \mathrm{O}, \mathrm{Mg}$ and $\mathrm{Si}$ ) along with the number of absorption lines observed for each species.

Abundance uncertainties have been estimated using a similar method to that described in Paper V. Random uncertainties have been estimated from the scatter in the line-by-line abundances of each element and hence include both uncertainties in the $E W$ measurements and random errors in the atomic data. For those objects where only one line was observed, the random uncertainties were assumed to be equivalent to the scatter in the oxygen abundances. If only a few oxygen features were observed (and hence it was not possible to estimate this scatter), a random uncertainty of 0.2 dex was assumed, which is typical for stars with many observed oxygen lines. Systematic errors in the abundances were estimated by considering the uncertainties in each of the atmospheric parameters. For stars where the effective temperature was constrained from the silicon ionization balance an uncertainty of $1000 \mathrm{~K}$ was adopted, otherwise an uncertainty of $1500 \mathrm{~K}$ was assumed. However in contrast to Paper V we consider that the effective temperature and surface gravity estimates are correlated in order to improve our error estimates, with an increase of $1000 \mathrm{~K}$ resulting in a higher gravity estimate of 0.1 dex. In addition we adopt measurement errors in the surface gravity to be 0.05 dex for stars with gravities of less than 3.0 dex and 0.1 dex otherwise. Similarly the microturbulence was better estimated at small values and an uncertainty of $3 \mathrm{~km} \mathrm{~s}^{-1}$ has been adopted for values of less than $10 \mathrm{~km} \mathrm{~s}^{-1}$ with $5 \mathrm{~km} \mathrm{~s}^{-1}$ being taken otherwise. The systematic and random uncertainties were summed in quadrature to give the uncertainties quoted in Table 2.

The derived carbon abundances are known to be susceptible to non-LTE effects (see Sigut 1996; Nieva \& Przybilla 2006, 2008) and these are not fully taken into account by the rather simplistic model ion that was included in our TLUSTY calculations. Paper V discussed adding a correction of 0.34 dex (from Lennon et al. 2003) to the derived carbon abundances from the $4267 \AA$ line. This was to achieve better agreement between the estimated carbon abundance of B-type stars and those of H II regions. However, since the non-LTE effects will be temperature dependent, such a correction will also be temperature dependent. Here we adopt a more sophisticated method.

Sigut (1996) utilises a more complex model atom than does our TLUSTY grid. We have compared abundances from our grid with those calculated by Sigut over a range of effective temperatures by adopting the equivalent widths that Sigut used to reproduce a carbon abundance of 8.55 dex (Grevesse et al. 1994). The results are shown in Fig. 4. It is clear that there are differences of up to 0.3 dex and we have applied corrections to the abundances from the $4267 \AA$ line in order to reproduce the temperature independent trend of Sigut. Our adopted carbon abundances are solely from these corrected estimates for this line. We note that the calculations of Sigut apply only at a surface gravity of $4.00 \mathrm{dex}$, a microturbulence of $5 \mathrm{~km} \mathrm{~s}^{-1}$ and solar metallicity and hence the extrapolation to other parameters and metallicity regimes should be treated with caution.

\subsection{Chemical compositions}

In each metallicity regime, the sample of stars is composed of objects in at least two fields or clusters (see Sect. 2). Papers V 
Table 3. Mean abundances (excluding upper limits) for each metallicity region given on the scale $12+\log [\mathrm{X} / \mathrm{H}]^{1}$. Solar and $\mathrm{H}$ II region abundances are given for comparison.

\begin{tabular}{lccccccc}
\hline \hline \multirow{2}{*}{ Species } & \multirow{2}{*}{ Solar $^{2}$} & \multicolumn{2}{c}{ Galaxy } & \multicolumn{2}{c}{ LMC } & \multicolumn{2}{c}{ SMC } \\
& & B-stars & H II regions & B-stars & H II regions $^{4}$ & B-stars & H II regions $^{4}$ \\
\hline Carbon $^{5}$ & 8.39 & $8.00 \pm 0.19(56)$ & - & $7.70 \pm 0.19(132)$ & $7.81 \pm 0.22$ & $7.30 \pm 0.28(81)$ & $7.20 \pm 0.02$ \\
Nitrogen $^{6}$ & 7.78 & $7.62 \pm 0.12(45)$ & $7.57 \pm 0.30$ & $7.13 \pm 0.29(83)$ & $6.92 \pm 0.14$ & $7.24 \pm 0.31(36)$ & $6.56 \pm 0.07$ \\
Oxygen $_{\text {Magnesium }}$ & 8.66 & $8.63 \pm 0.16(54)$ & $8.70 \pm 0.30$ & $8.34 \pm 0.13(133)$ & $8.37 \pm 0.09$ & $7.99 \pm 0.21(80)$ & $8.02 \pm 0.04$ \\
Silicon & 7.51 & $7.25 \pm 0.17(52)$ & & $7.05 \pm 0.14(134)$ & & $6.72 \pm 0.18(72)$ & \\
\hline
\end{tabular}

${ }^{1}$ Values in brackets indicate the number of stars for which abundances were estimated.

${ }^{2}$ Asplund et al. (2005).

${ }^{3}$ Average of solar neighbourhood regions from Shaver et al. (1983).

${ }^{4}$ Kurt \& Dufour (1998).

${ }^{5}$ The carbon abundances have been corrected, see text.

${ }^{6}$ Supergiants have been excluded from the mean nitrogen abundances.

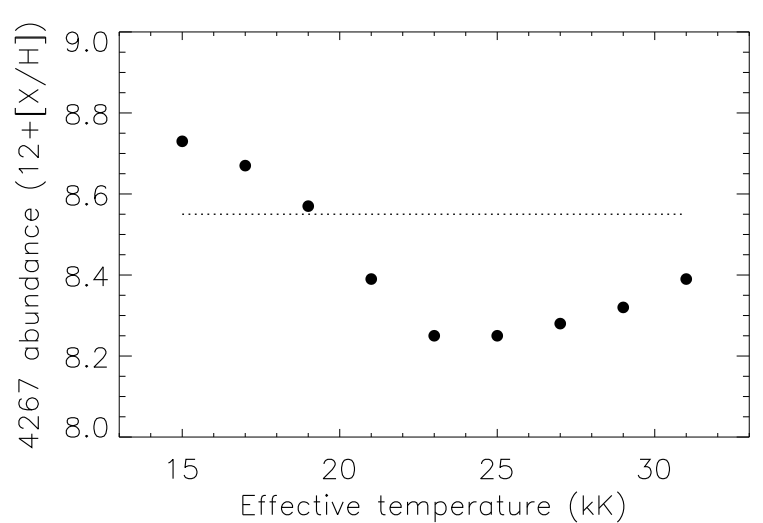

Fig. 4. Comparison between the abundance estimated from the C II 4267 $\AA$ line using the TLUSTY grid (points) and that adopted by Sigut (1996; for a gravity of $4.00 \mathrm{dex}$, microturbulence of $5 \mathrm{~km} \mathrm{~s}^{-1}$ at solar metallicity; dashed line). Corrections have been applied to remove the temperature dependence of the TLUSTY carbon abundances.

and VI have presented a detailed abundance analysis of the narrow-lined targets in each field and show that, within the same metallicity regime, there are no systematic differences between the abundances from each field. This is consistent with each Magellanic Cloud being homogeneously mixed and with the Galactic clusters having similar galactocentric distances (the Galaxy is known to have a metallicity gradient, see, for example, Rolleston et al. 2000). In this context we combine the targets in each metallicity regime in order to increase the sample size. In Table 3 the average abundances for each metallicity regime are given. These are compared to the baseline chemical compositions from $\mathrm{H}$ II regions and the solar composition as recently determined by Asplund et al. (2005).

The mean oxygen abundances of the three regions are in excellent agreement with the $\mathrm{H}$ II region abundances. Our Galactic nitrogen abundance is also in good agreement with that from $\mathrm{H}$ II regions. However, the mean nitrogen abundance of the SMC from B-type stars is clearly larger than that from H II regions. The LMC nitrogen abundance from B-type stars is also greater than that from H II regions and, although these mean LMC values can be reconciled within the uncertainties, it is clear from Sect. 4 that there is a mix of normal and significantly enriched stars. In addition, the scatter in the stellar nitrogen abundance is larger than that for other elements and we conclude that this is not dominated by observational scatter, but due to significant abundance differences amongst the sample stars (see Sect. 4).
Our Magellanic Cloud carbon abundances are in good agreement with those from $\mathrm{H}$ II regions implying that our correction to the carbon abundance may be reasonable. However, obtaining a Galactic $\mathrm{H}$ II region carbon abundance from the literature is highly problematic. For example, Dufour et al. (1982) give a value of 8.46 dex for the Orion nebula whereas Walter et al. (1992) find a range of 7.94-8.78 dex for the same region depending on the consideration of temperature fluctuations. Given the additional problem of determining accurate stellar carbon abundances it is difficult to confirm the validity of our mean Galactic carbon abundances.

\section{Results}

Chemical compositions are presented for 53 Galactic and 96 SMC stars and compared with the results for the 135 LMC stars from Paper VII. In order to investigate the role of rotational mixing, a large population of fast rotators is necessary. Our targets have projected rotational velocities up to $\sim 300 \mathrm{~km} \mathrm{~s}^{-1}$ and hence are well suited to such a comparison. In particular the nitrogen abundance can be used as a tracer of surface enrichments and our discussion will only consider this element. While in principle associated carbon depletions would be expected, these would be of the order of the uncertainties in our carbon abundance estimates. We adopt the baseline nitrogen abundances of the three regions from Paper V, which are based on $\mathrm{H}$ II regions and unenriched B-type stars. In Paper VII the distribution of the nitrogen abundances of the LMC stars as a function of their projected rotational velocity was described. In Fig. 5 the plot from Paper VII is shown and the principle conclusions are summarized below.

- The majority of the core-hydrogen burning stars show little nitrogen enrichment and the efficiency of rotational mixing in the evolutionary models has been constrained to fit the mean nitrogen abundance of this population. Many of the nitrogen abundances for the faster rotators are upper limits. We find that the rotational mixing models provide a reasonable fit to half of our core-hydrogen burning data-set, but is in conflict with the rest of the stars (Groups 1 and 2).

- Group 1 consists of non-enriched fast rotators, of which many are in the late stage of core-hydrogen burning (with relatively low gravity) and hence a significant nitrogen enrichment would be expected. This group has been redefined since Paper VII. The observed sample was magnitude selected thereby excluding low mass stars close to the beginning of their core-hydrogen burning phase. We therefore 


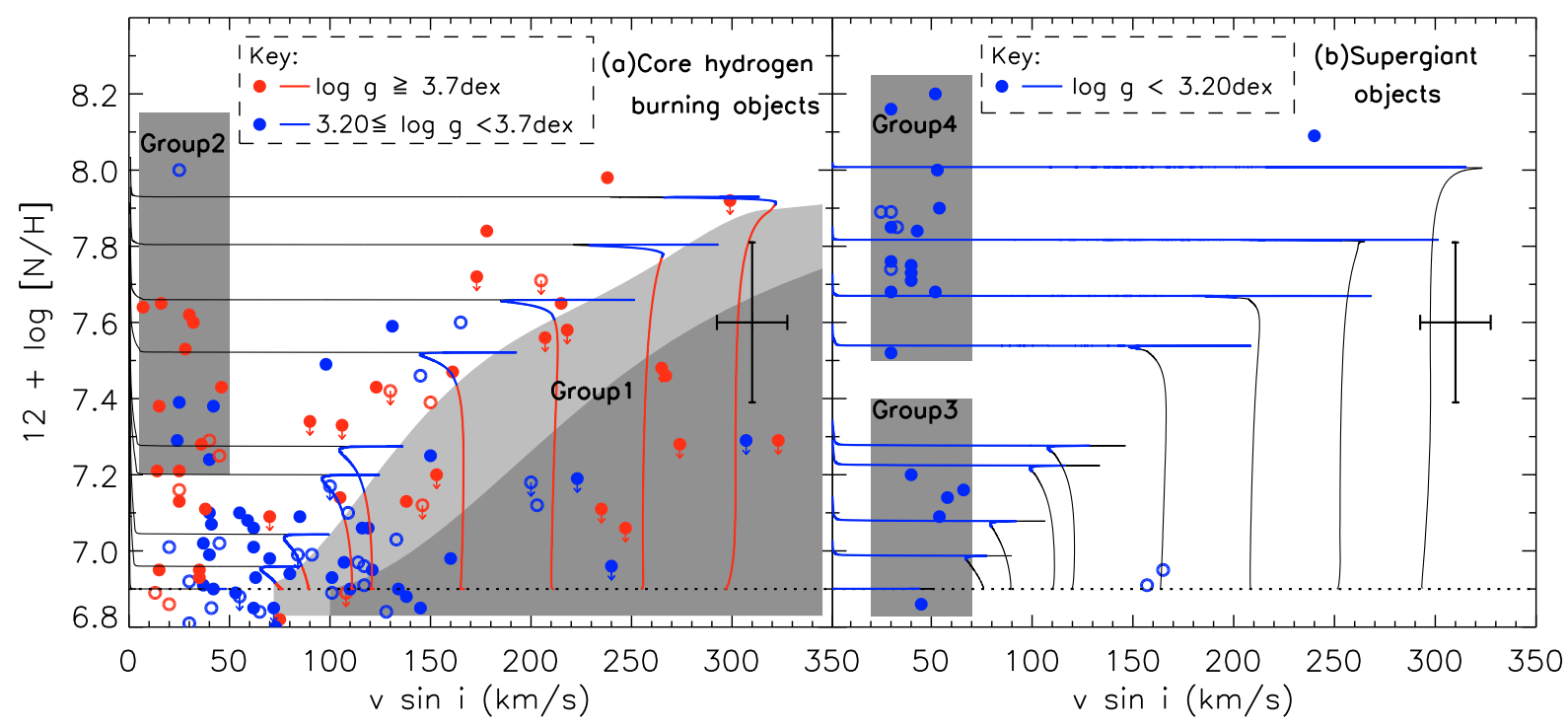

Fig. 5. Nitrogen abundance $(12+\log [\mathrm{N} / \mathrm{H}])$ as a function of projected rotational velocity for the $\mathrm{LMC}$ sample of stars. This plot has been taken from Paper VII. The mean error bars of the nitrogen abundances and projected rotational velocities are indicated. Core-hydrogen burning objects a) and supergiants b) are defined based on their surface gravity. In panel a) the core-hydrogen burning objects are sub-divided to indicate objects in the first part or latter part of the core hydrogen burning phase, red and blue symbols respectively, approximately $70 \%$ and $30 \%$ of the main-sequence lifetime. Open symbols represent radial velocity variables. Downward arrows indicate upper limits to the nitrogen abundances. The dotted line indicates the LMC baseline nitrogen abundance as determined from H II region analyses and unmixed B-type stars (Paper V). The rotational velocity of the evolutionary models have been scaled by $\pi / 4$ to take into account random angles of inclination. The individual groups of objects have been discussed in Paper VII and are summarized in the text although we have redefined the Group 1 stars (see text). The evolutionary models in a) and b) have initial masses of $13 M_{\odot}$ and $19 M_{\odot}$ respectively. We note that a minor error in the temperature scale adopted in Paper VII was discovered and hence several of the points in the figure have been corrected. However this is an effectively negligible correction as the mean nitrogen abundance and the number of stars in each group remain unchanged.

should be biased against young unenriched stars, indicated by the dark shaded area of Group 1 (plotted for the mean mass of the sample, $13 M_{\odot}$ ). In addition to these stars, the observed evolved stars (blue points) in the light grey region are unexpected as these stars are predicted to have higher nitrogen abundances for their rotational velocities and evolutionary state. Group 1 comprises $\sim 30 \%$ of the non-binary sample, two thirds of which are close to the end of their corehydrogen burning lifetime (blue points).

- The large number of significantly enriched stars in Group 2 (a further $20 \%$ of the sample) cannot be explained as fast rotators seen at low inclination angles if these are randomly distributed. The models do not predict strong rotational mixing for slowly rotating stars and in Paper VII we postulate that these may be magnetic stars, equivalent to those discussed by Morel et al. (2006, 2008). We note that we are not referring to magnetic fields produced by dynamo action (Spruit 2002) which would be expected to be present in all stars, but rather fields of fossil origin (see Morel et al. 2008 and references therein). Huang \& Gies (2006b) also suggest that remnant magnetic fields can account for the peculiar helium abundances of a subset of their sample.

- The supergiant objects appear to be split into two distinct groups. The stars in Group 3 (see Fig. 5) have enrichments consistent with the core-hydrogen burning phase which might be expected if they are their direct descendants. However, the supergiants shown in Group 4 have highly enriched atmospheres. It is postulated that these stars have previously gone through a red supergiant phase. As discussed in Paper VII, our stellar evolution models do predict sufficient enrichment in the red supergiant phase, but the location of these Group 4 objects on the Hertzsprung-Russell diagram cannot be reproduced.
The above summary of the results presented in Paper VII assumes that selection effects in our sample do not introduce a significant observational bias. One such source of bias could be that the rapidly rotating stars will appear brighter when viewed pole on (with low projected rotational velocities) than at high inclination angles (with large projected rotational velocities). This is due to the pole caps being hotter than the equatorial regions as discussed by von Zeipel (1924). Such a bias (see also Maeder 2009) would affect the Magellanic Cloud samples (which are magnitude limited) and lead to an oversampling of rapidly rotating pole-on stars (which would populate Group 2 discussed above) and an under-sampling of stars with large projected rotational velocities that could undergo significant nitrogen enhancement and be the precursors to the Group 4 supergiants.

We have investigated this possibility as follows. Martayan et al. (2006, 2007) estimated mean equatorial velocities for B-type stars (i.e. excluding the Be-type stars as has been done for the current sample) in the SMC and LMC ranging from approximately 120 to $160 \mathrm{~km} \mathrm{~s}^{-1}$ with similar values being found in Paper IV. For Galactic targets, the estimated mean values are similar (Strom et al. 2005; Huang \& Gies 2006a, Paper III). For the observed B-type stellar populations discussed by Martayan et al. only $8 \%$ of their targets have projected rotational velocities of greater than $300 \mathrm{~km} \mathrm{~s}^{-1}$, in both NGC 330 and NGC 2004. Additionally only two stars (out of a sample of more than 300 objects) were found with a projected rotational velocity of greater than $400 \mathrm{~km} \mathrm{~s}^{-1}$. Similar results were found for the Magellanic Cloud samples considered here. We have therefore adopted a rotational velocity of $400 \mathrm{~km} \mathrm{~s}^{-1}$ as representative of the most rapidly rotating B-type stars and hence appropriate for investigating the possible consequences of the von Zeipel effect for our sample. We note that this velocity is consistent or larger than the values that have been assumed when modelling 
rotational mixing in B-type stars (see, for example, Heger \& Langer 2000; Meynet \& Maeder 2000).

Adopting typical physical parameters for an early-B-type main sequence star $\left(T_{\text {eff }}=25000 \mathrm{~K} ; M=13 M_{\odot} ; R(\right.$ pole $)=$ $5 R_{\odot}$ ) yields a ratio of the equatorial rotational velocity to the breakup velocity of $v_{\mathrm{eq}} / v$ (crit) $=0.7$ for $v_{\mathrm{eq}}=400 \mathrm{~km} \mathrm{~s}^{-1}$. For this ratio we estimate the change in $B$ magnitude between poleon $\left(i=0^{\circ}\right)$ and equator-on $\left(i=90^{\circ}\right)$ views to be only 0.36 mag, using the methodology outlined by Townsend et al. (2004). This $\Delta B$ estimate has very little dependence on B spectral subtype, and hence should be representative of our entire B-type mainsequence sample.

The above example implies that there could be a bias in our sample in the sense that we would preferentially observe fast rotators at low inclination angles. However it should be noted that such objects will be intrinsically very rare for two reasons. Firstly the results of Martayan et al. (2006) and Paper IV indicate that rapidly rotating LMC B-type stars will be intrinsically rare, with those having rotational velocities greater than or equal to $400 \mathrm{~km} \mathrm{~s}^{-1}$ making up less than $1 \%$ of an unbiased sample of LMC targets. Additionally the Group 2 stars in Fig. 5 have projected rotational velocities of less than $50 \mathrm{~km} \mathrm{~s}^{-1}$. Assuming that they have a unique rotational velocity of $400 \mathrm{~km} \mathrm{~s}^{-1}$, the probability of observing them at such a low angle of inclination would be less than $1 \%$; for higher rotational velocities the probability would be even smaller. Hence we estimate that for an LMC population of early B-type stars, the fraction of stars with a rotational velocity greater than or equal to $400 \mathrm{~km} \mathrm{~s}^{-1}$ but observed to have a projected rotational velocity of less than $50 \mathrm{~km} \mathrm{~s}^{-1}$ would be of the order of one in ten thousand. It was the rareness of such objects that formed the basis of the assumption in Paper VII that most of the Group 2 stars that make up one in five of our sample must be slowly rotating.

We can estimate the amount of bias in our Group 2 sample based on the simulation discussed above. Our approach has been to exclude all stars from the Group 2 sample that lie within 0.36 mag of our photometric cutoff. Hence if all Group 2 stars were rotating at $400 \mathrm{~km} \mathrm{~s}^{-1}$, there would be no bias with respect to similar objects observed equatorially $\left(i=90^{\circ}\right)$. Exclusion of such stars reduces the LMC Group 2 sample from seventeen to thirteen stars. Given our sample size, we would expect on average approximately 0.01 Group 2 stars in our sample compared with the 13 targets found in our corrected sample. Another approach is just to assume that our Group 2 stars are fast rotators and that the von Zeipel effect is far larger than we have calculated. Then we would expect there to be a corresponding very large number of stars (of the order of $10^{5}$ ) with large projected rotational velocities below our photometric cut-off. The LMC investigation of Martayan et al. (2006) went approximately two magnitudes fainter than our survey but neither in their photometry nor in their projected rotational velocity estimates is there any evidence for these objects.

We note that in our simulations, we have made a number of arbitrary assumptions, for example a single rotational velocity. However relaxing this assumption would be unlikely to change our conclusions. For example, although there would be more stars with lower rotational velocities, the von Zeipel effect would be smaller; conversely more rapid rotators would be very rare as would be the probability of observing them at low angles of inclination. In conclusion, we find that even at rotational velocities as high as $400 \mathrm{~km} \mathrm{~s}^{-1}$, the pole on rotators will appear brighter in the B band by less than $0.4 \mathrm{mag}$, whilst only approximately one in ten thousand LMC B-type stars should have such rotational velocities with a projected rotational velocity consistent with our Group 2 sample. On the basis of these simulations, we conclude that almost all, if not all, LMC Group 2 stars must be intrinsically slow rotators.

\subsection{Evolutionary models}

As described in Paper VII stellar evolutionary models have been generated to fit the observed LMC data. Briefly, these models have been based on the code of Yoon et al. (2006) and include the effects of rotation (Heger et al. 2000) and angular momentum transport via magnetic torques (Spruit 2002). The code has been updated to include the mass-loss rates of Vink et al. (2001). The magnetically induced chemical diffusion term is not considered as it is not observationally supported (Spruit 2006). An appropriate chemical composition has been chosen, in particular with the light elements set to the observed values from Paper V. The overshooting parameter ( 0.335 of the pressure scale height) has been adopted to reproduce the observed trend of rotational velocities from Paper IV. The efficiency parameter for rotational mixing has been set as described in Paper VII. In summary, LMC models were calibrated to best fit the observed data set.

Further evolutionary models have been generated to represent Galactic and SMC compositions with the overshooting and rotational mixing efficiency parameters unchanged as these parameters are not predicted to be metallicity dependent. The light element composition appropriate for these metallicities has again been adopted from Paper V. The full grid of evolutionary models will be discussed in a forthcoming paper (Brott et al. in prep.). Here we select models with masses appropriate for our early B-type stellar sample and which cover a range of rotational velocities suitable to make comparisons between the current theory and our observational data set.

\subsection{Galactic stars}

We plot the nitrogen abundances of the Galactic sample as a function of projected rotational velocity in Fig. 6 together with our evolutionary models. Figure 6 a shows that within the abundance uncertainties no trend of nitrogen abundance with rotational velocity is observed. The evolutionary models suggest that for stars with initial rotational velocities of $250 \mathrm{~km} \mathrm{~s}^{-1}$ nitrogen abundances up to $\sim 8.0$ dex should be observed.

The lack of any significant enrichment amongst the corehydrogen burning stars is surprising. In a population similar to our observed LMC sample we would expect some nitrogen-rich fast rotators, as - even assuming rotational mixing does not occur - those stars could then be interpreted as spun-up mass gainers in close binaries. Also, we would expect some enriched slow rotators as found in the LMC, as such stars have been identified in the Galaxy recently by Morel et al. (2006, 2008). Indeed, in the Galactic sample we have only included those stars with measurable nitrogen lines and while this introduces a bias against the fastest rotating Galactic stars, it also provides a bias towards the most enriched stars.

The lack of nitrogen-enriched stars may be due to the majority of the core-hydrogen burning stars in our sample being relatively unevolved. As rotational mixing is a continuous process, its effect will be most prominent toward core-hydrogen exhaustion. Also close binary mass transfer is expected much more frequently toward the end of hydrogen burning, since the expansion of core-hydrogen burning stars accelerates in the later stages. Finally, the process which leads to nitrogen enhancement in the slowly rotating early B-type stars is as yet unknown. That we 


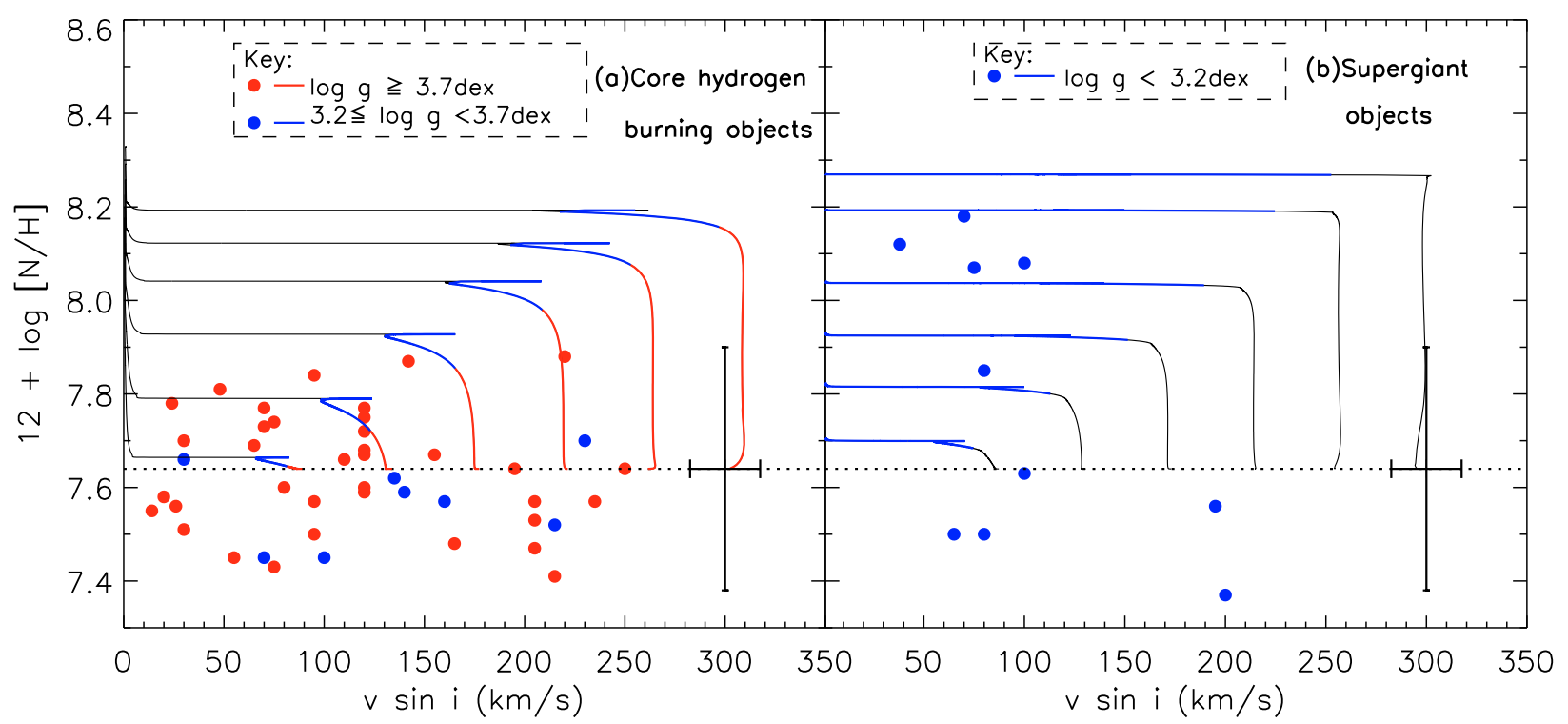

Fig. 6. Nitrogen abundance $(12+\log [\mathrm{N} / \mathrm{H}])$ as a function of projected rotational velocity for the Galactic sample of stars. Symbols are equivalent to those in Fig. 5. Evolutionary models are plotted for 10 and $20 M_{\odot}$ in panels a) and b) to represent the mean mass of the two samples.

do not find such stars here might imply that the enrichment time scale is of the order of the main sequence life time.

The hypothesis that our sample stars are relatively unevolved is supported by the fact that in Fig. 6a, only eight stars have a surface gravity smaller than 3.7 dex. Note that the limiting gravity between the two classes is set the same as for the LMC corehydrogen burners, but that the Galactic stars are more metalrich and have larger radii (and hence lower gravities) than LMC stars of the same mass and evolutionary stage. Despite that, the LMC sample has a majority of stars with $\log g<3$.7. It should be noted that the observations of the Magellanic Cloud samples have been selected via a magnitude cut (Paper II) which prohibits the observation of zero-age main-sequence stars at the lower masses of the sample. For the Galactic sample this magnitude cut is low enough that the unevolved main sequence stars are not excluded. Additionally there are no blue supergiants with a mass close to the mean sample mass of our core-H burning stars $\left(\sim 10 M_{\odot}\right)$ in our Galactic supergiant sample (Fig. 6b). Finally our Galactic sample is mainly composed of cluster stars whereas in the Magellanic Cloud we sample more of the field population which may contain more evolved stars.

Our sample shows seven Galactic core-hydrogen burning objects with low surface gravities (i.e. close to the end of the core-hydrogen burning phase; blue points) and nitrogen abundances lower than that predicted for their evolutionary stage. The two apparently fast rotating supergiants with low nitrogen abundances could also be placed in this group within the uncertainty in their surface gravities. According to the models we would expect these objects to be nitrogen rich, in particular the fastest rotators. These objects may be analogous to the Group 1 stars shown in Fig. 5 and make up $\sim 20 \%$ of the core-hydrogen burning sample, although if we adopt the maximum error several objects could be reconciled with the models. While this group is smaller than that observed in the LMC sample, given the less evolved nature of our Galactic sample this is not unexpected.

In order to determine if we should observe a group of objects similar to Group 2 in Fig. 5a we have added the mean absolute enrichment of those objects to the Galactic baseline nitrogen abundance (on a logarithmic scale) and find that such a group of objects would appear to have a normal nitrogen abundance within the abundance uncertainties. However, Morel et al. (2008) find slowly rotating magnetic Galactic stars with nitrogen abundances up to 8.0 dex. In Paper VII we considered these stars to be analogous to the Group 2 stars in the LMC and linked fossil magnetic fields to chemical mixing, although the mixing mechanism is unknown. It is unclear why we do not see these stars in our Galactic sample. The Morel et al. sample contains some bias towards such stars and this bias is not present in our Galactic data set. Additionally our Galactic sample is predominantly composed of cluster stars whereas field stars dominate the Magellanic Cloud samples. Wolff et al. (2007) have provided evidence that cluster stars rotate significantly faster than field stars, which is why our Galactic sample contains fewer slower rotators than our Magellanic Cloud samples (see Fig. 2). They relate this to differences in the star formation process between cluster and field stars.

We also find some evidence for a difference between field and cluster stars in our LMC sample. The LMC targets lie towards the clusters NGC 2004 and N 11. In the former case almost our entire sample lies beyond the cluster radius and hence is probably a field star population, whereas for $\mathrm{N} 11$ our stars are taken from across the association and the field. While $\sim 65 \%$ of our LMC sample of core-hydrogen burning non-binary stars is taken from the NGC 2004 targets, 80\% of the Group 2 stars are from the field of NGC 2004. This may indicate that the Group 2 stars are more likely to occur in the field population and hence explain the lack of such stars in our Galactic cluster sample. However, the significance of the lack of highly mixed slowly rotating core hydrogen burning stars cannot be truly tested.

Figure 6b suggests that there are two groups of supergiants with different degrees of nitrogen enrichment, in agreement with the situation in the LMC sample, Fig. 5b, although the number of stars in the Galactic sample is limited. Given that no corehydrogen burning Galactic objects are observed with nitrogen abundance consistent with the highly enriched supergiants, it appears unlikely that these objects have evolved directly from such a phase and hence they should not be used to constrain the models of core-hydrogen burning stars. The supergiant sample is further discussed in Sect. 4.4. 

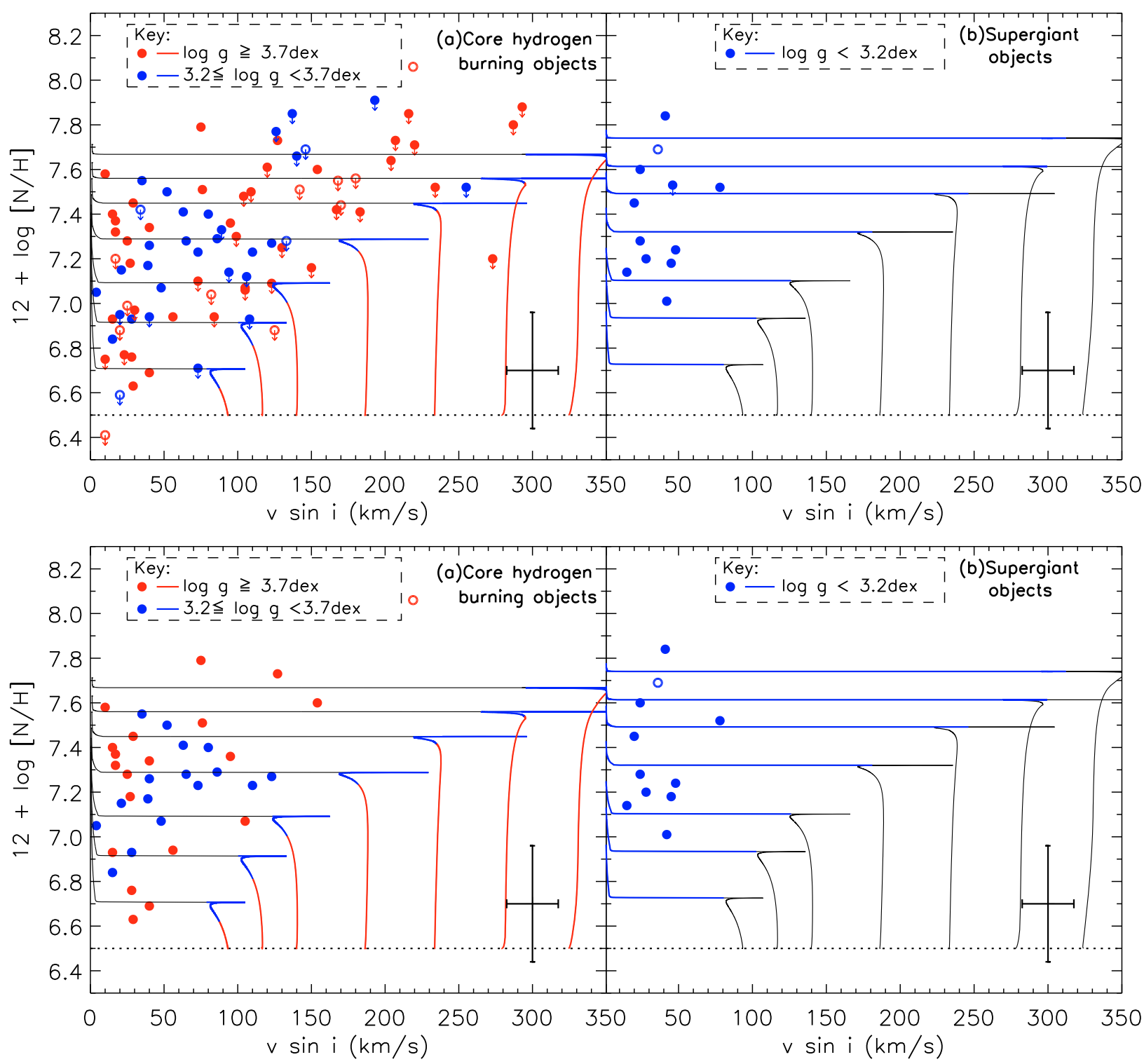

Fig. 7. Nitrogen abundance $(12+\log [\mathrm{N} / \mathrm{H}])$ as a function of projected rotational velocity for the SMC sample of stars. Symbols are equivalent to those in Fig. 5. The lower panel is equivalent to the upper panel except that upper limits to the nitrogen abundances have been removed. Evolutionary models are plotted for 12 and $13 M_{\odot}$ in panels a) and b).

\subsection{SMC stars}

In Fig. 7 we plot the nitrogen abundance as a function of projected rotational velocity for the SMC sample of stars. From the upper panel there appears to be a strong correlation between these two quantities, as would be expected from the theory of rotational mixing. However, for the majority of stars with rotational velocities greater than $100 \mathrm{~km} \mathrm{~s}^{-1}$ only upper limits to the nitrogen abundances have been estimated and such a limit will be correlated with the projected rotational velocity leading to the apparent trend. If we remove these upper limits (lower panel) this correlation disappears. The lack of absolute nitrogen abundances for these fast rotators prevents the observation of a group of stars similar to the Group 1 stars of Fig. 5a. We note that although the estimation of upper limits to the nitrogen abundance is not metallicity dependent, our SMC data is of lower quality (Sect. 2) and hence higher upper limits are derived, particularly for the fastest rotators.

A comparison between Figs. 7a and 5a shows that while the majority of the LMC stars have undergone little or no enrichment, many stars in the SMC appear to be significantly enriched. Our mean SMC nitrogen abundance is actually higher than that of the LMC despite the SMC having a lower baseline abundance. However, given that nitrogen lines can only be observed for abundances greater than approximately 6.9 dex for even a moderately rotating star, it is difficult to test the significance of this observation and it should be noted that the mean values are consistent within the uncertainties. Nevertheless, we find 30 of our 70 non-binary core-hydrogen burning stars (excluding upper limits) have such high estimates that, even adopting an average error bar of $\pm 0.26 \mathrm{dex}$, they have nitrogen abundances that are inconsistent with the core-hydrogen burning regime of the stellar evolution tracks in Fig. 7a. This is obviously a lower limit as stars with upper limits to their nitrogen abundances could also populate this group if their abundances are indeed higher than the model predictions.

The mean nitrogen abundance of the SMC sample is $7.24 \mathrm{dex}$ and to reproduce this abundance evolutionary tracks with an initial rotational velocity of $\sim 200 \mathrm{~km} \mathrm{~s}^{-1}$ are required. The 
probabilities of a star rotating at $200 \mathrm{~km} \mathrm{~s}^{-1}$ appearing to have projected rotational velocities of less than 100 and $50 \mathrm{~km} \mathrm{~s}^{-1}$ are $13 \%$ and $3 \%$ respectively. We observe 19 and 12 stars with such projected rotational velocities but with nitrogen abundances consistent, within their uncertainties, with a $200 \mathrm{~km} \mathrm{~s}^{-1}$ evolutionary track. It is therefore statistically very unlikely that these groups are populated by fast rotators with low angles of inclination. For example, in order to have 12 stars rotating at $200 \mathrm{~km} \mathrm{~s}^{-1}$ and appearing with rotational velocities of less than $50 \mathrm{~km} \mathrm{~s}^{-1}$ a population of 400 stars would be required with $\sim 260$ of these having projected rotational velocities in the range $150-200 \mathrm{~km} \mathrm{~s}^{-1}$. Such a population clearly does not exist in the upper panel of Fig. 7 and, although Be-type stars are excluded, there is no bias against normal B-type stars with these velocities. We can therefore conclude that the enriched stars with low projected rotational velocities have intrinsically low rotational velocities. This is clearly in conflict with the theory of rotational mixing and these stars may be analogous to the LMC stars designated as Group 2 in Fig. 5.

There appears to be a gap between the Group 2 stars in the LMC (Fig. 5a) and higher velocity stars. Such a gap is not clear in the SMC sample. The significance of this gap is unknown, but if it is real it implies that, whatever the process that is enriching the Group 2 stars, it is efficient at higher velocities in the SMC sample. Alternatively the sample may be populated by two mixing processes, the first being that producing the LMC Group 2 stars while the latter process mixes all the SMC stars to a similar nitrogen abundance. Indeed, it should be noted that the majority of the Group 2 LMC stars are high gravity stars and the same holds true for the analogous sample in the SMC (e.g. projected rotational velocities of less than $50 \mathrm{~km} \mathrm{~s}^{-1}$ and nitrogen abundances greater than 7.2 dex), which is also dominated by a large population of high gravity stars.

Our sample could again be biased by the von Zeipel effect (1924). To estimate the degree of bias, we have again considered an object with an equatorial rotational velocity of $400 \mathrm{~km} \mathrm{~s}^{-1}$. As discussed above the Group 2 objects are less clearly defined in the SMC sample. However adopting the criteria of a logarithmic surface gravity greater than $\geq 3.2$ dex, projected rotational velocity of $\leq 50 \mathrm{~km} \mathrm{~s}^{-1}$ and a nitrogen abundance of $\geq 7.2 \mathrm{dex}$ (corresponding to a nitrogen enhancement of more than 0.6 dex), we find 11 stars. Removing those within 0.36 mag of our $B$-band magnitude cut-off reduces the sample size to 8 stars. SMC B-type stars have intrinsically larger rotational velocities than their LMC or Galactic counterparts (Martayan et al. 2007; Paper IV) and we would expect approximately $3 \%$ to have values of $400 \mathrm{~km} \mathrm{~s}^{-1}$ or more. Again less than $1 \%$ of them will have an inclination angle leading to a projected rotational velocity of $\leq 50 \mathrm{~km} \mathrm{~s}^{-1}$. Hence we would expect that approximately one in four thousand SMC B-type stars would have a rotational velocity of $\geq 400 \mathrm{~km} \mathrm{~s}^{-1}$ and a projected rotational velocity of $\leq 50 \mathrm{~km} \mathrm{~s}^{-1}$. In turn, for our SMC sample size, we would then expect on average 0.02 such stars to lie in the same region as the Group 2 LMC stars. Alternatively, if we assume that all our Group 2 sample are rapidly rotating, we would expect a corresponding population of approximately 30000 rapidly rotating stars with larger inclination angles. Martayan et al. (2007) study of the SMC cluster NGC 330 goes approximately two magnitudes fainter than our sample and again there is no evidence for this population. Hence we conclude that at least several (and probably most, or all) of the nitrogen-enhanced SMC targets with low projected rotational velocities must be intrinsically slow rotators.
In both Figs. $5 \mathrm{~b}$ and $6 \mathrm{~b}$ the supergiant sample consisted of two groups of objects, one mildly enriched (or unenriched) group consistent with the core-hydrogen burning stars and a highly enriched group. This is not observed in the SMC sample (Fig. 7b). However, given that almost all the stars in the corehydrogen burning sample are significantly enriched, in contrast to the Galactic and LMC samples, this might mask such a distribution. Although the mass of the SMC supergiants $\left(\sim 13 M_{\odot}\right)$ is lower than that of the Galaxy and LMC samples $\left(\sim 20 M_{\odot}\right)$ the correlation of nitrogen abundance with mass is expected to be small (compare evolutionary tracks in Fig. 5a and b) and none is observed in the LMC sample of stars which cover a wide range of masses. Nevertheless there are a number of supergiant objects with nitrogen abundances greater than $7.6 \mathrm{dex}$, which is higher than the nitrogen abundance generally observed during the core hydrogen burning phase, indicating that part of the mixing in these supergiants may occur after the hydrogen burning phase.

\subsection{Supergiants}

As discussed above, the supergiants typically show two groups, one highly enriched group and one less enriched (or normal) group, although the effect is less obvious in the SMC sample. In Fig. 8 the nitrogen abundance is plotted against the surface gravity (evolutionary status) for each of the three metallicity regimes. It is clear that the lowest gravity supergiants have the highest nitrogen abundances at all metallicities.

As discussed in Paper VII and illustrated in Fig. 8, the amount of surface nitrogen enrichment that is obtained during the first dredge-up phase can reasonably reproduce the observed nitrogen abundances of the enriched supergiants in the LMC. This suggests that some of our blue supergiants have previously evolved through a red supergiant stage, with their surfaces enriched through dredge-up. Given that there is little predicted trend with mass and velocity (less than 0.3 dex) for the amount of enrichment during this red supergiant stage, this would imply that all stars which have gone through such a phase should have similar nitrogen abundances regardless of their initial parameters while stars which have not gone through this phase would have lower abundances. Such bimodal distributions are clear in the Galactic and LMC samples. The expected nitrogen enrichment from dredge-up is in excellent agreement with the LMC observations and, while it is slightly higher than our Galactic observations, within the uncertainties it is consistent. The models predict that the nitrogen enrichment from dredge-up in the SMC sample is not significantly greater than that observed for the majority of our core-hydrogen burning sample and hence a bi-modal distribution would not be easily observed. The predicted nitrogen abundance after first dredge-up is lower than that observed for the most enriched SMC supergiants but again, within the uncertainties, the hypothesis that the most enriched supergiants have gone through a red supergiant phase is not unreasonable.

However, in order for our enriched supergiants to be explained by such a process it would be necessary for those stars to return to hotter temperatures to allow us to observe them in the blue supergiant stage on a Hertzsprung-Russell diagram. Such a blue-loop was, for example, proposed as the mechanism to explain the progenitor of SN1987A. Whilst these loops are not predicted by our evolutionary models, those in the Geneva models (see, for example, Maeder \& Meynet 2001) do not extend to high enough masses or to hot enough temperatures to reproduce our observations. If the single star nature of these supergiants can be observationally confirmed it is clear that more extensive blue loops need to be predicted by the evolutionary models. Indeed, 

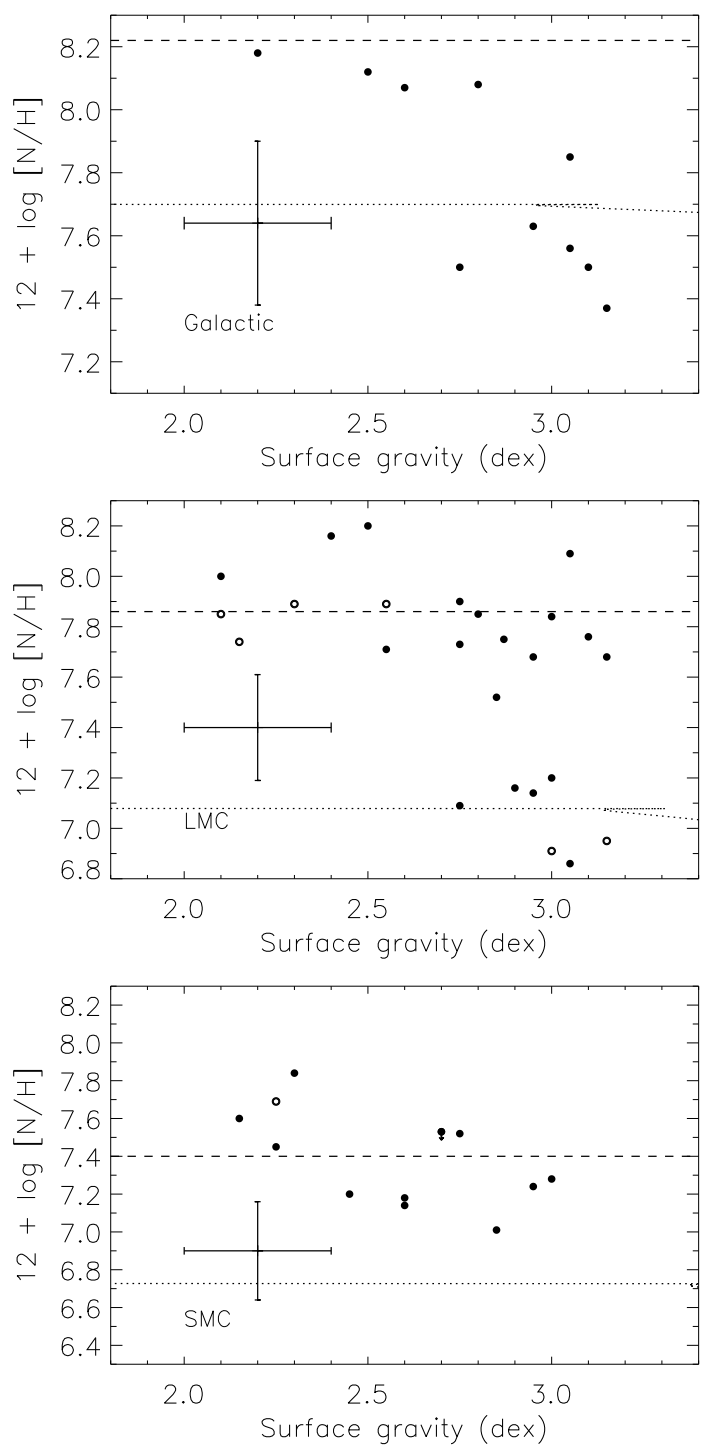

Fig. 8. Nitrogen abundances of the supergiants in each region. Open symbols represent radial velocity variables. Error bars represent the mean uncertainty in the nitrogen abundance for each of the samples and an uncertainty of 0.2 dex in surface gravity. The dotted line represents the evolutionary track for a model at the mean mass of the sample and a moderate rotational velocity $\left(100 \mathrm{~km} \mathrm{~s}^{-1}\right)$ although as the mainsequence rotational velocity of these stars is unknown it is not possible to make a detailed comparison with the models. The dashed line represents the nitrogen abundances achieved during the first dredge-up for the same models, although it should be noted that the models do not predict a return to the temperatures and gravities representative for our observations.

such a scenario may also help to bring the expected and observed number of blue supergiants into better agreement (Paper IV).

Venn \& Przybilla (2003, a re-analysis of previous work, Venn 1999) have presented similar nitrogen abundances for A-F type supergiants in the Galaxy and SMC and drawn similar conclusions to those presented above.

\section{Conclusions}

Chemical compositions are presented for $\sim 50$ Galactic and 100 SMC early B-type stars which cover a broad range of rotational velocities and complement previously published results for 130 LMC stars of similar spectral types for which new observationally constrained evolutionary models had been generated. We compare our new observational data sets with the LMC results.

\subsection{Core-hydrogen burning objects}

In both the Magellanic Cloud populations we find a significant excess of slowly rotating nitrogen enriched stars that cannot be explained within the context of rotational mixing, at least for a random distribution of inclination angles; $20 \%$ in the LMC and $\sim 40 \%$ in the SMC. In the Galactic sample of the FLAMES survey, such stars are not found, although they are present in other Galactic samples (Morel et al. 2006, 2008). While this may be attributed to the early evolutionary stage of the majority of the FLAMES Galactic stars, it would constrain the time scale of the enrichment process for this group - the physics of which is unknown - to be comparable to the main-sequence lifetime.

The LMC sample contains a significant group of stars which are rapidly rotating, but show no significant nitrogen enhancement. Due to the magnitude cut used in our target selection, the zero-age main-sequence is not well sampled at low mass. Hence if only single stars are considered and rotational mixing is assumed to be valid, such nitrogen normal rapid rotators are not predicted by rotational mixing models. A significant fraction of these stars have gravities that imply that they are close to the end of the core-hydrogen burning phase. The Galactic sample also reveals several fast rotating low gravity stars although they constitute a smaller fraction probably due to the less evolved nature of the Galactic sample. In the SMC, the rather high upper limits on nitrogen abundances for rapid rotators does not allow for their identification.

Finally, the LMC sample contains about $20 \%$ of stars which are rather rapidly rotating and nitrogen enriched. Those stars could either be rotationally mixed single stars (Maeder et al. 2009; Brott et al. 2009) if the non-enriched rapid rotators are all binary products), or the spun-up mass gainer in mass transfer binaries (Langer et al. 2008). While some such stars may be contained in the SMC sample, they are not found in our Galactic sample. This may be due to the rather unevolved nature of this sample although it should be noted that our Galactic sample contains fewer slow rotators compared to the Magellanic Cloud sample.

The differences in data quality and mean evolutionary state of the samples do not allow clear trends in relative numbers of the various groups of core-hydrogen burning stars to be obtained for each metallicity regime.

\subsection{Supergiants}

In all three metallicity regimes a population of highly enriched supergiant objects have been observed, which are unlikely to be the direct descendants of rotationally mixed core-hydrogen burning stars. The predicted amount of nitrogen after first dredge up reasonably reproduces their nitrogen abundances. However, the position of these stars in the Hertzsprung-Russell diagram cannot be reproduced by our evolutionary models and, if the single star nature of these objects can be confirmed, argues for the occurrence of blue loops at hotter temperatures and higher masses than currently predicted.

In summary we find that both the Galactic and SMC samples support the conclusions made in Paper VII. It should also be noted that we find no evidence for an excess of binary systems in the groups of objects that are in conflict with the rotating 
single star models although our observations do not allow for complete binary identification. Our results imply that a new nitrogen enrichment process (or processes) other than rotational mixing must be important in massive star evolution. Furthermore, close binary effects may be needed to understand the rapid rotators. Whether rotational mixing is required to understand our results remains an open question at this time, but can be answered by a rigourous study of the binary fraction in those groups of stars which appear to contradict the theory.

Acknowledgements. We are grateful to staff from the European Southern Observatory for assistance in obtaining the data. This work, conducted as part of the award "Understanding the lives of massive stars from birth to supernovae" (SJS) made under the European Heads of Research Councils and European Science Foundation EURYI (European Young Investigator) Awards scheme, was supported by funds from the Participating Organisations of EURYI and the EC Sixth Framework Programme. SJS also acknowledges the Leverhulme Trust. Additionally we acknowledge financial support from STFC (UK Science and Technology Facilities Council), NWO (Netherlands Science Foundation) and DEL (Department of Education and Learning in Northern Ireland).

\section{References}

Asplund, M., Grevesse, N., \& Sauval, A. J. 2005, in Cosmic Abundances as Records of Stellar Evolution and Nucleosynthesis, ed. T. G. Barnes III, \& F. N. Bash (San Francisco: ASP), ASP Conf. Ser., 336, 25

Bouret, J.-C., Lanz, T., Hillier, D. J., et al. 2003, ApJ, 595, 1182

Brott, I., Hunter, I., de Koter, A., et al. 2009, Comm. in Asteroseismology, Contribution to the Proceedings of the 38th LIAC [arXiv: 0810.2348]

Crowther, P. A. Hillier, D. J., Evans, C. J., et al. 2002, ApJ, 579, 774

Crowther, P. A., Lennon, D. J., \& Walborn, N. R. 2006, A\&A, 446, 279

Dufour, R. J., Shields, G. A., \& Talbot, R. J. Jr. 1982, ApJ, 252, 461

Dufton, P. L. 1972, A\&A, 16, 301

Dufton, P. L., Ryans, R. S. I, Trundle, C., et al. 2005, A\&A, 434, 1125

Dufton, P. L., Smartt, S. J., Lee, J.-K., et al. 2006, A\&A, 457, 265 (Paper III)

Daflon, S., Cunha, K., Butler, K., \& Smith, V. V. 2001, ApJ, 563, 325

Evans, C. J., Smartt, S. J., Lee, J.-K., et al. 2005, A\&A, 437, 467 (Paper I)

Evans, C. J., Lennon, D. J., Smartt, S. J., \& Trundle, C. 2006, A\&A, 456, 632 (Paper II)

Gies, D. R., \& Lambert, D. L. 1992, ApJ, 387, 673

Garnett, D. R. 1999, in New Views of the Magellanic Clouds, ed. Y.-H. Chu, N. Suntzeff, J. Hesser, \& D. Bohlender, IAU Symp., 190, 266

Grevesse, N., Sauval, A. J. \& Blomme, R. 1994, in Infrared Solar Physics, ed. D. M. Rabin, J. T. Jefferies, \& C. Lindsey (Dordrech: Kluwer), IAU Symp., 154,531

Heger, A., \& Langer, N. 2000, ApJ, 544, 1016

Heger, A., Langer, N., \& Woosley, S. E. 2000, ApJ, 528, 368

Howarth, I. D., \& Smith, K. C. 2001, MNRAS, 327, 353

Huang, W., \& Gies, D. R. 2006a, ApJ, 648, 580

Huang, W., \& Gies, D. R. 2006b, ApJ, 648, 591

Hubeny, I., \& Lanz, T. 1995, ApJ, 439, 875

Hunter, I., Dufton, P. L., Smartt, S. J., et al. 2007, A\&A, 466, 277 (Paper V)

Hunter, I., Lennon, D. J., Dufton, P. L., et al. 2008a, A\&A, 479, 541 (Paper IV)

Hunter, I., Brott, I., Lennon, D. J., et al. 2008b, ApJ, 676, L29 (Paper VII)
Kilian, J. 1991, A\&A, 262, 320 385, 143

Korn, A. J., Keller, S. C., Kaufer, A., et al. 2002, A\&A, 385, 143

Korn, A. J., Nieva, M. F., Daflon, S., \& Cunha, K. 2005, ApJ, 633, 899

Kurt, C. M., \& Dufour, R. J. 1998, in Texas-Mexico: Astrophysical plasmas near and far, ed. R. J. Dufour, \& Torres-Peimbert, Rev. Mex. Astron. Astrofis. Conf. Ser., 7, 202

Langer, N., Cantiello, M., Yoon, S. C., et al., 2008, IAU-Symp., 250, 167

Lennon, D. J., Dufton, P. L. Keenan, F. P., \& Holmgren, D. E. 1991, A\&A, 246, 175

Lennon, D. J., Dufton, P. L., \& Crowley, C. 2003, A\&A, 317, 87

Maeder, A., \& Meynet, G. 2001, A\&A, 373, 555

Maeder, A., Meynet, G., Ekstrom, S., \& Georgy, C. 2009, Comm. in Asteroseismology, Contribution to the Proceedings of the 38th LIAC [arXiv: 0810.0657]

Martins, F., Schaerer, D., \& Hillier, D. J. 2002, A\&A, 382, 999

Martins, F., Schaerer, D., \& Hillier, D. J. 2005, A\&A, 436, 1049

Martayan, C., Frémat, Y., Hubert, A.-M., et al. 2006, A\&A, 452, 273

Martayan, C., Frémat, Y., Hubert, A.-M., et al. 2007, A\&A, 462, 683

Massey, P., Bresolin, F., Kudritzki, R. P., Puls, J., \& Pauldrach, A. W. A. 2004, ApJ, 608, 1001

Massey, P., Puls, J., Pauldrach, A. W. A., et al. 2005, ApJ, 627, 477

Meynet, G., \& Maeder, A. 2000, A\&A, 361, 101

Meynet, G., \& Maeder, A. 2005, A\&A, 429, 581

Mokiem, M. R., de Koter, A., Evans, C. J., et al. 2006, A\&A, 456, 1131

Mokiem, M. R., de Koter, A., Evans, C. J., et al. 2007, A\&A, 465, 1003

Morel, T., Butler, K., Aerts, C., Neiner, C. \& Briquet, M. 2006, A\&A, 457, 651

Morel, T., Hubrig, S., \& Briquet, M. 2008, A\&A, 481, 453

Nieva, M. F., \& Przybilla, N. 2006, ApJ, 639, 39

Nieva, M. F., \& Przybilla, N. 2008, A\&A, 481, 199

Rolleston, W. R. J., Smartt, S. J., Dufton, P. L., \& Ryans, R. S. I. 2000, A\&A, 363, 537

Shaver, P. A., McGee, R. X., Newton, L. M., Danks, A. C., \& Pottasch, S. R. 1983, MNRAS, 204, 53

Sigut, T. A. A. 1996, ApJ, 473, 452

Simón-Díaz, S., \& Herrero, A. 2007, A\&A, 468, 1063

Slettebak, A. 1968, ApJ 154, 933

Spruit, H. C. 2002, A\&A, 381, 923

Spruit, H. C. 2006 [arXiv: astro-ph/0607164]

Strom, S. E., Wolff, S. C., \& Dror, D. H. A. 2005, ApJ, 129, 809

Townsend, R. H. D., Owocki, S. P., \& Howarth, I. D. 2004, MNRAS, 350, 189

Trundle, C., \& Lennon, D. J. 2005, A\&A, 434, 677

Trundle, C., Dufton, P. L., Hunter, I., et al. 2007, A\&A, 471, 625 (Paper VI)

Villamariz, M. R., \& Herrero, A. 2005, A\&A, 442, 263

Venn, K. A. 1999, ApJ, 518, 405

Venn, K. A., \& Przybilla, N. 2003, in CNO in the universe, ed. C. Charbonnel, D. Schaerer, \& G. Meynet (Valais, Switzerland: ASP), ASP Conf. Ser., 304, 20

Vink, J. S., \& de Koter, A. 2005, A\&A, 442, 587

Vink, J. S., de Koter, A., \& Lamers, H. J. G. L. M. 2001, A\&A, 369, 574

von Zeipel, H. 1924, MNRAS, 84, 655

Vrancken, M., Hensberge, H., David, M., \& Verschueren, W. 1997, A\&A, 320, 878

Walborn, N. R. 1970, ApJ, 161, 149

Wolff, S. C., Strom, S. E., \& Dror, D. 2007, AJ, 133, 1092

Walter, D. K., Dufour, R. J., \& Hester, J. J. 1992, ApJ, 397, 196

Woosley, S. E., \& Heger, A. 2006, ApJ, 637, 914

Yoon, S.-C., \& Langer, N. 2005, A\&A, 443, 643

Yoon, S.-C., Langer, N., \& Norman, C. 2006, A\&A, 460, 199 
I. Hunter et al.: Chemical compositions of B-type stars, Online Material p 1
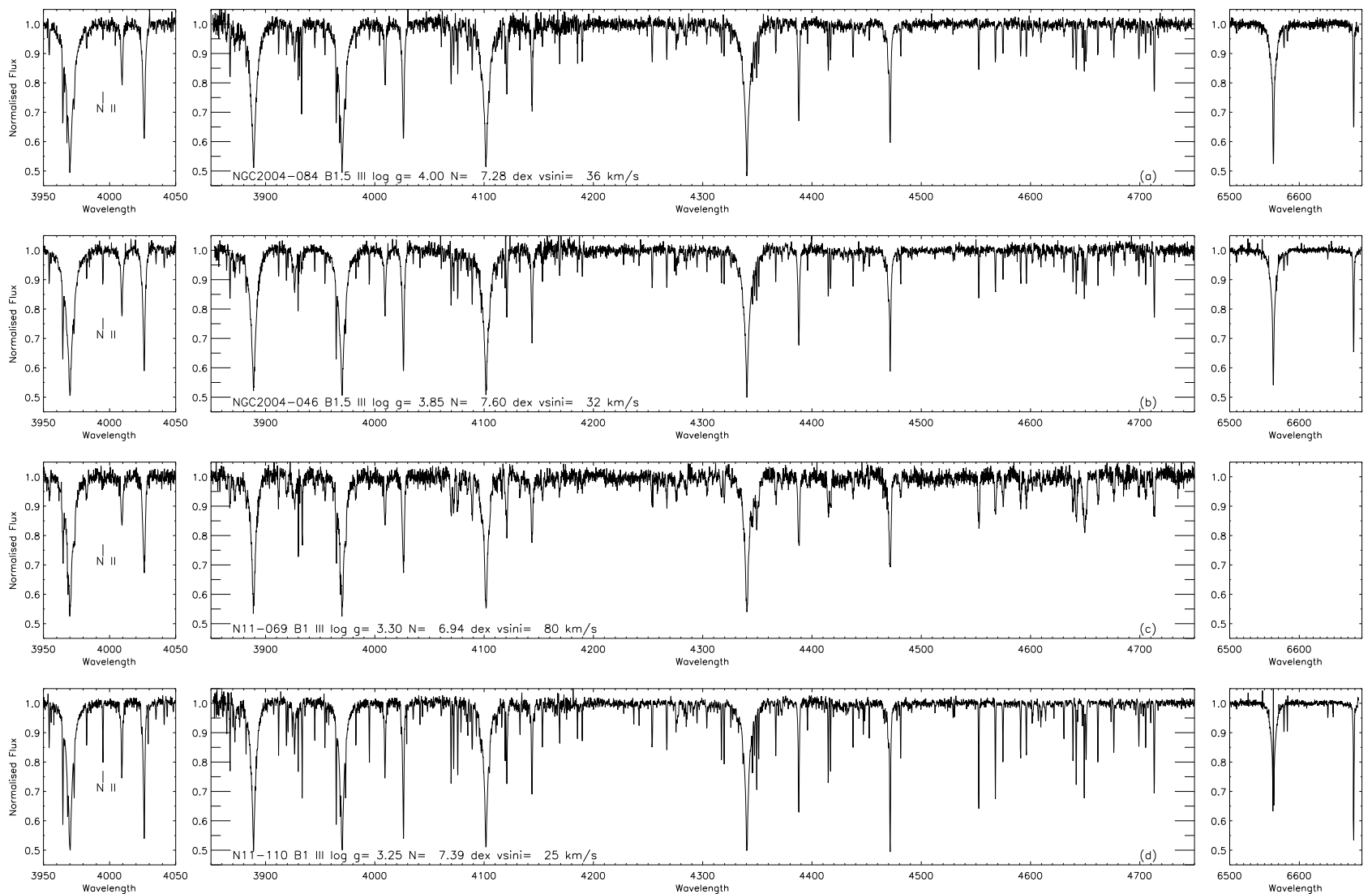

Fig. 1. Comparison of the spectra for a subset of nitrogen rich and nitrogen normal LMC stars. The spectral region containing the N II $3995 \AA$ line is expanded (left panel) and the line is indicated. The spectral type, surface gravity, nitrogen abundance and projected rotational velocity of these stars are indicated in the panels. Other than the strength of the nitrogen lines there are no systematic differences between the spectra of stars of the same spectral type, surface gravity and projected rotational velocity. 
I. Hunter et al.: Chemical compositions of B-type stars, Online Material p 2
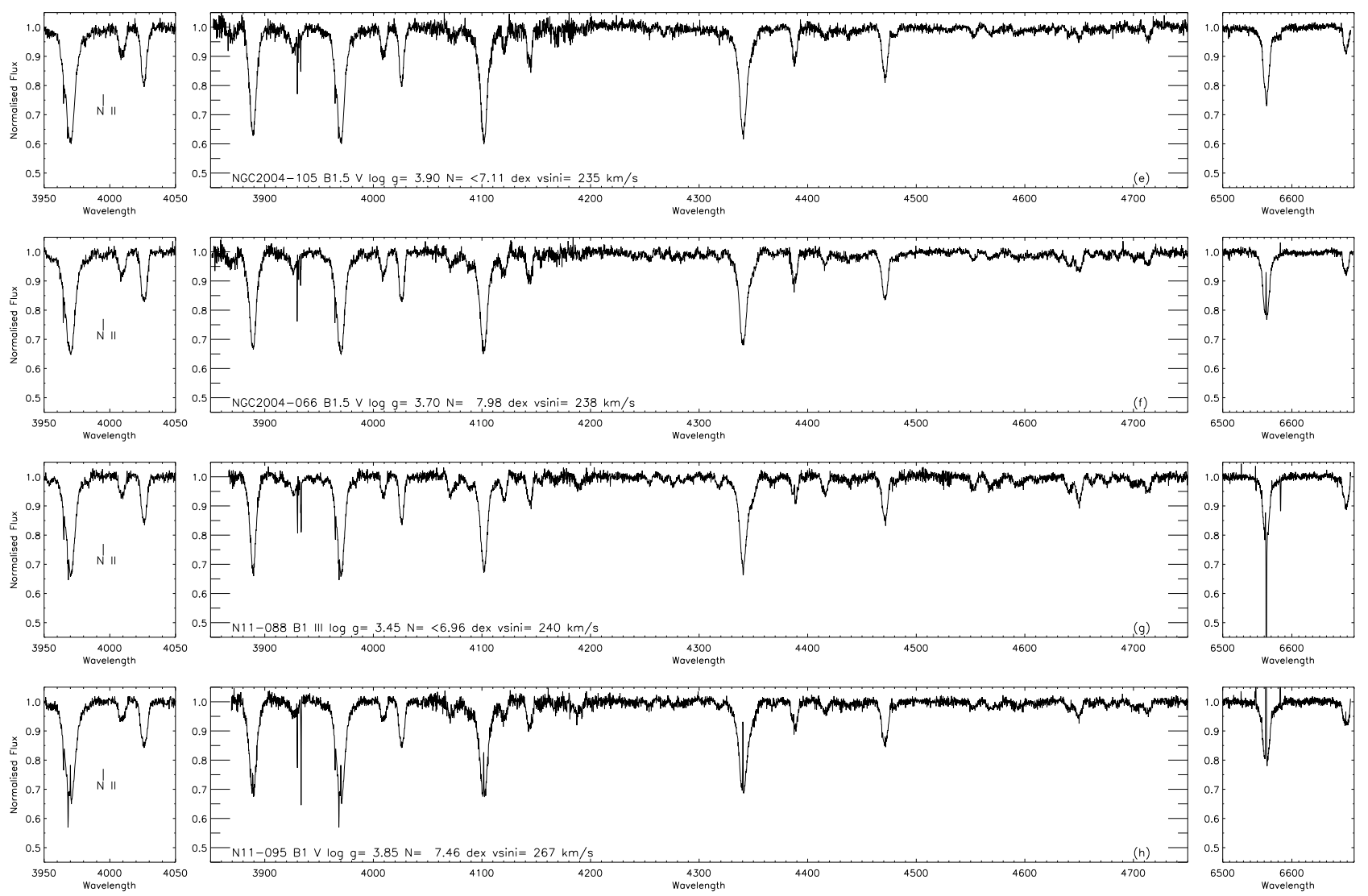

Fig. 1. continued.
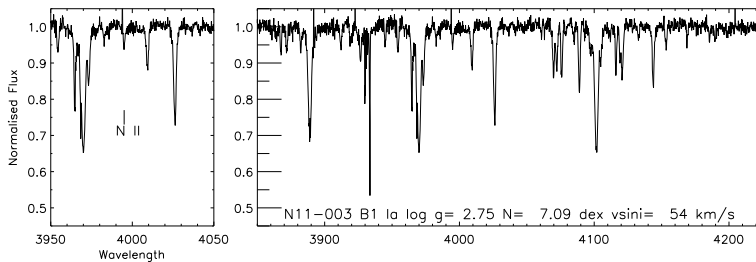

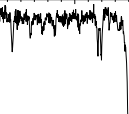
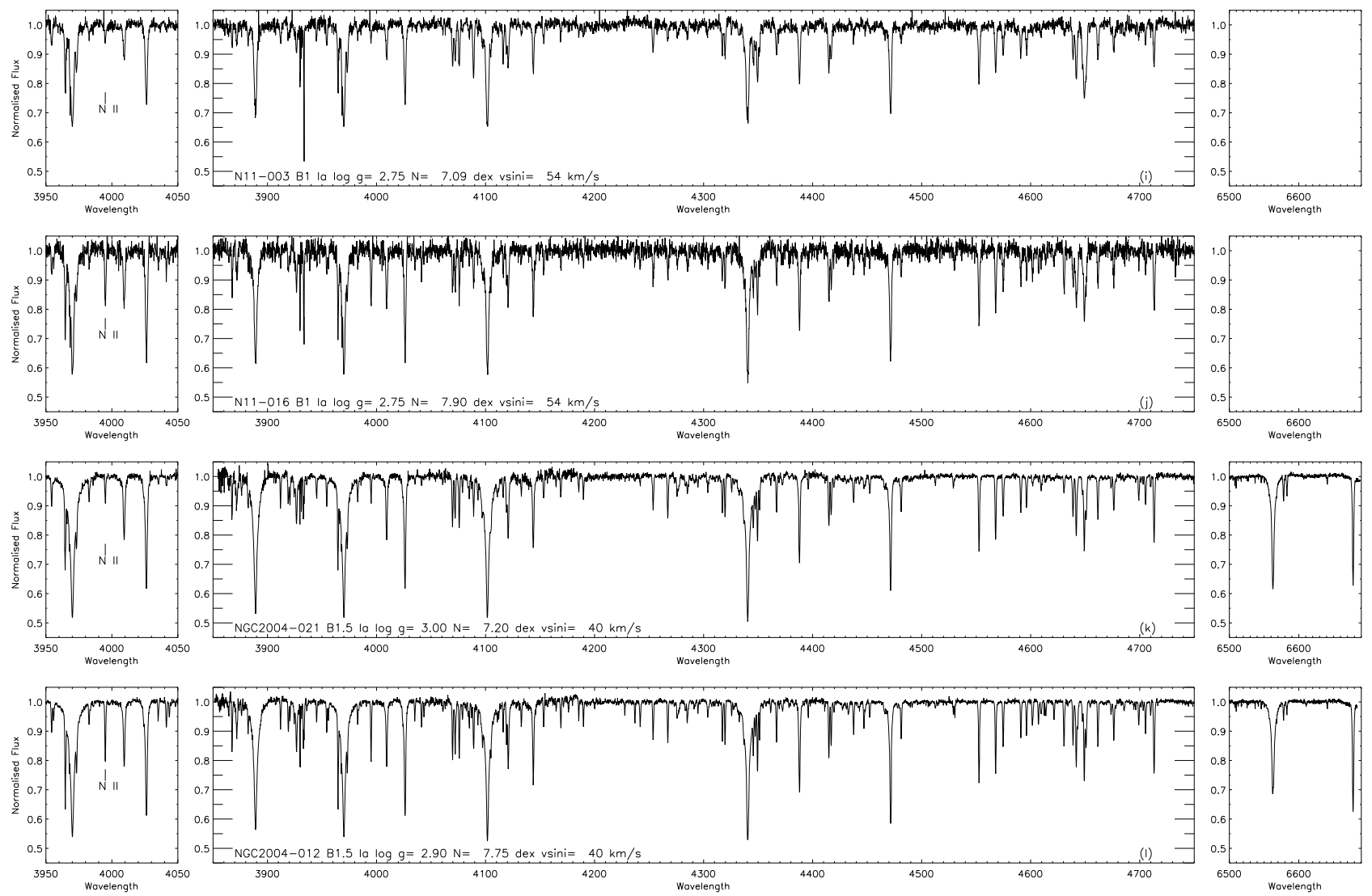

Fig. 1. continued. 
I. Hunter et al.: Chemical compositions of B-type stars, Online Material p 3

Table 1. Equivalent width measurements and derived abundances for the metal lines observed in the spectra of our sample stars.

\begin{tabular}{|c|c|c|c|c|c|c|c|c|c|c|c|}
\hline \multirow[t]{2}{*}{ Species } & \multirow[t]{2}{*}{ Wavelength } & \multicolumn{2}{|c|}{ NGC 6611-001 } & \multicolumn{2}{|c|}{ NGC 6611-006 } & \multicolumn{2}{|c|}{ NGC 6611-012 } & \multicolumn{2}{|c|}{ NGC 6611-020 } & \multicolumn{2}{|c|}{ NGC 6611-021 } \\
\hline & & $E W$ & Abund & $E W$ & Abund & $E W$ & Abund & $E W$ & Abund & $E W$ & Abund \\
\hline C II & 4267.00 & 88 & 8.06 & 91 & 8.14 & 134 & 8.19 & 111 & 7.98 & 112 & 7.92 \\
\hline N II & 3955.85 & - & - & - & - & - & - & - & - & - & - \\
\hline N II & 3995.00 & 42 & 7.52 & 47 & 7.60 & 74 & 7.50 & - & - & 64 & 7.43 \\
\hline N II & 4447.03 & 48 & 7.85 & 26 & 7.54 & - & - & 49 & 7.67 & 35 & 7.45 \\
\hline N II & 4601.48 & 42 & 8.13 & - & - & - & - & - & - & - & - \\
\hline N II & 4613.86 & - & - & - & - & - & - & - & - & - & - \\
\hline N II & 4630.54 & 78 & 7.99 & 36 & 7.60 & - & - & 96 & 8.09 & 60 & 7.64 \\
\hline N II & 4643.08 & - & - & - & - & - & - & - & - & - & - \\
\hline O II & 3912.00 & - & - & 61 & 8.44 & 103 & 8.61 & - & - & 95 & 8.83 \\
\hline O II & 3919.30 & - & - & - & - & - & - & - & - & - & - \\
\hline O II & 3945.04 & 45 & 8.58 & 34 & 8.43 & 65 & 8.59 & 68 & 8.74 & 45 & 8.52 \\
\hline O II & 3954.36 & - & - & 63 & 8.47 & 90 & 8.52 & - & - & 77 & 8.73 \\
\hline O II & 3982.71 & - & - & 29 & 8.35 & - & - & - & - & 54 & 8.78 \\
\hline O II & 4069.00 & - & - & - & - & 247 & 9.06 & - & - & - & - \\
\hline O II & 4072.15 & - & - & 97 & 8.34 & 127 & 8.56 & - & - & 85 & 8.44 \\
\hline O II & 4075.86 & 133 & 8.39 & 109 & 8.26 & 145 & 8.56 & - & - & 86 & 8.27 \\
\hline O II & 4078.84 & - & - & 35 & 8.51 & 37 & 8.27 & - & - & - & - \\
\hline O II & 4132.80 & - & - & 48 & 8.49 & 83 & 8.71 & - & - & 60 & 8.70 \\
\hline O II & 4156.53 & - & - & - & - & 30 & 8.74 & - & - & - & - \\
\hline O II & 4185.44 & - & - & 52 & 8.29 & - & - & - & - & 37 & 8.13 \\
\hline O II & 4317.00 & - & - & 83 & 8.94 & 95 & 8.69 & - & - & 81 & 8.86 \\
\hline O II & 4319.63 & - & - & 76 & 8.88 & 99 & 8.75 & - & - & 76 & 8.76 \\
\hline O II & 4325.76 & - & - & - & - & - & - & - & - & - & - \\
\hline O II & 4349.43 & - & - & - & - & - & - & - & - & 90 & 8.73 \\
\hline O II & 4351.00 & - & - & 65 & 8.16 & - & - & - & - & 79 & 8.52 \\
\hline O II & 4353.58 & - & - & 10 & 8.07 & - & - & - & - & - & - \\
\hline O II & 4366.00 & - & - & - & - & 72 & 8.33 & - & - & 68 & 8.52 \\
\hline O II & 4369.27 & - & - & - & - & - & - & - & - & - & - \\
\hline O II & 4395.94 & - & - & 33 & 8.51 & 41 & 8.42 & - & - & 59 & 8.96 \\
\hline O II & 4414.90 & - & - & 96 & 8.51 & 118 & 8.31 & 127 & 8.55 & 112 & 8.60 \\
\hline O II & 4416.98 & - & - & 85 & 8.65 & 95 & 8.38 & 79 & 8.33 & 101 & 8.80 \\
\hline O II & 4443.01 & - & - & 17 & 8.34 & - & - & - & - & - & - \\
\hline O II & 4452.38 & - & - & 28 & 8.68 & 59 & 8.73 & - & - & - & - \\
\hline O II & 4590.97 & - & - & 76 & 8.37 & 110 & 8.46 & 97 & 8.52 & 89 & 8.68 \\
\hline O II & 4596.18 & - & - & 71 & 8.43 & 112 & 8.55 & 76 & 8.31 & 85 & 8.62 \\
\hline O II & 4638.86 & - & - & 84 & 8.76 & 120 & 8.74 & - & - & 77 & 8.63 \\
\hline O II & 4641.81 & - & - & 130 & 8.72 & 169 & 8.72 & - & - & 96 & 8.47 \\
\hline O II & 4650.00 & - & - & 205 & 8.60 & - & - & - & - & - & - \\
\hline O II & 4661.63 & - & - & 87 & 8.72 & 93 & 8.40 & - & - & 80 & 8.61 \\
\hline O II & 4673.73 & - & - & - & - & - & - & - & - & - & - \\
\hline O II & 4676.23 & - & - & 69 & 8.68 & - & - & - & - & 63 & 8.46 \\
\hline O II & 4699.00 & - & - & 76 & 8.38 & 97 & 8.27 & - & - & 87 & 8.39 \\
\hline O II & 4705.35 & 43 & 8.10 & 75 & 8.38 & 77 & 8.19 & 57 & 8.04 & 75 & 8.44 \\
\hline O II & 4710.00 & - & - & 32 & 8.74 & - & - & - & - & - & - \\
\hline Mg II & 4481.00 & 101 & 7.36 & 97 & 7.36 & 102 & 7.26 & 139 & 7.60 & 97 & 7.24 \\
\hline Si II & 4128.05 & - & - & - & - & - & - & - & - & - & - \\
\hline Si II & 4130.89 & - & - & - & - & - & - & - & - & - & - \\
\hline Si III & 4552.62 & 166 & 7.38 & 139 & 7.37 & 181 & 7.42 & 145 & 7.38 & 122 & 7.35 \\
\hline Si III & 4567.84 & 141 & 7.48 & 117 & 7.45 & 163 & 7.54 & 136 & 7.56 & 95 & 7.26 \\
\hline Si III & 4574.75 & 79 & 7.56 & 56 & 7.41 & 93 & 7.38 & 84 & 7.47 & 80 & 7.60 \\
\hline Si IV & 4088.90 & - & - & - & - & - & - & - & - & - & - \\
\hline Si IV & 4116.10 & 251 & 7.47 & 194 & 7.40 & 98 & 7.41 & - & - & 41 & 7.41 \\
\hline Si IV & 4212.00 & - & - & 61 & 7.38 & - & - & - & - & - & - \\
\hline
\end{tabular}


I. Hunter et al.: Chemical compositions of B-type stars, Online Material p 4

Table 1. continued.

\begin{tabular}{|c|c|c|c|c|c|c|c|c|c|c|c|}
\hline \multirow[t]{2}{*}{ Species } & \multirow[t]{2}{*}{ Wavelength } & \multicolumn{2}{|c|}{ NGC 6611-025 } & \multicolumn{2}{|c|}{ NGC 6611-030 } & \multicolumn{2}{|c|}{ NGC 6611-032 } & \multicolumn{2}{|c|}{ NGC 6611-033 } & \multicolumn{2}{|c|}{ NGC 6611-035 } \\
\hline & & $E W$ & Abund & $E W$ & Abund & $E W$ & Abund & $E W$ & Abund & $E W$ & Abund \\
\hline C II & 4267.00 & 157 & 8.19 & 218 & 8.35 & 209 & 8.32 & 160 & 8.19 & 114 & 8.00 \\
\hline N II & 3955.85 & - & - & - & - & - & - & - & - & - & - \\
\hline N II & 3995.00 & 82 & 7.58 & 69 & 7.53 & 58 & 7.39 & 96 & 7.62 & - & - \\
\hline N II & 4447.03 & 64 & 7.85 & 40 & 7.63 & 40 & 7.65 & 56 & 7.65 & 54 & 7.74 \\
\hline N II & 4601.48 & 60 & 8.09 & 29 & 7.59 & 43 & 7.93 & - & - & 44 & 7.90 \\
\hline N II & 4613.86 & - & - & 34 & 7.95 & 28 & 7.83 & - & - & - & - \\
\hline N II & 4630.54 & 81 & 7.85 & 65 & 7.76 & 68 & 7.87 & 93 & 7.84 & 60 & 7.61 \\
\hline N II & 4643.08 & - & - & - & - & - & - & - & - & - & - \\
\hline O II & 3912.00 & 99 & 8.90 & 48 & 8.66 & 41 & 8.55 & 95 & 8.64 & 105 & 8.75 \\
\hline O II & 3919.30 & - & - & - & - & - & - & - & - & - & - \\
\hline O II & 3945.04 & - & - & 36 & 8.76 & - & - & 69 & 8.71 & - & - \\
\hline O II & 3954.36 & 73 & 8.68 & 55 & 8.87 & - & - & 74 & 8.43 & - & - \\
\hline O II & 3982.71 & - & - & 25 & 8.60 & - & - & 49 & 8.48 & - & - \\
\hline O II & 4069.00 & - & - & 79 & 8.44 & 67 & 8.31 & 157 & 8.46 & - & - \\
\hline O II & 4072.15 & - & - & 57 & 8.49 & 51 & 8.42 & 94 & 8.28 & - & - \\
\hline O II & 4075.86 & - & - & 76 & 8.66 & 93 & 8.99 & 134 & 8.60 & - & - \\
\hline O II & 4078.84 & - & - & 16 & 8.28 & - & - & 50 & 8.59 & - & - \\
\hline O II & 4132.80 & - & - & 27 & 8.45 & - & - & - & - & - & - \\
\hline O II & 4156.53 & - & - & - & - & - & - & - & - & - & - \\
\hline O II & 4185.44 & - & - & - & - & - & - & 61 & 8.40 & - & - \\
\hline O II & 4317.00 & 89 & 9.01 & 47 & 8.66 & 39 & 8.52 & 86 & 8.63 & - & - \\
\hline O II & 4319.63 & 95 & 9.12 & 43 & 8.58 & 34 & 8.40 & 85 & 8.62 & - & - \\
\hline O II & 4325.76 & - & - & 26 & 9.03 & - & - & - & - & - & - \\
\hline O II & 4349.43 & - & - & 45 & 8.51 & - & - & 105 & 8.55 & - & - \\
\hline O II & 4351.00 & - & - & 23 & 8.20 & - & - & 89 & 8.41 & - & - \\
\hline O II & 4353.58 & - & - & - & - & - & - & - & - & - & - \\
\hline O II & 4366.00 & - & - & 47 & 8.61 & - & - & 92 & 8.62 & - & - \\
\hline O II & 4369.27 & - & - & - & - & - & - & 28 & 8.41 & - & - \\
\hline O II & 4395.94 & 44 & 8.70 & 25 & 8.71 & - & - & 66 & 8.85 & - & - \\
\hline O II & 4414.90 & 102 & 8.50 & 66 & 8.47 & 42 & 8.06 & 123 & 8.44 & 114 & 8.42 \\
\hline O II & 4416.98 & 68 & 8.36 & 55 & 8.60 & 38 & 8.28 & 110 & 8.62 & 106 & 8.64 \\
\hline O II & 4443.01 & - & - & - & - & - & - & - & - & - & - \\
\hline O II & 4452.38 & - & - & 20 & 8.51 & - & - & 55 & 8.73 & - & - \\
\hline O II & 4590.97 & 88 & 8.69 & 41 & 8.42 & - & - & 93 & 8.44 & 124 & 8.88 \\
\hline O II & 4596.18 & 79 & 8.56 & 54 & 8.73 & 36 & 8.41 & 94 & 8.51 & 107 & 8.66 \\
\hline O II & 4638.86 & 91 & 8.85 & 51 & 8.68 & 43 & 8.55 & 97 & 8.61 & 100 & 8.71 \\
\hline O II & 4641.81 & 139 & 9.03 & 64 & 8.48 & 63 & 8.52 & 119 & 8.38 & 150 & 8.81 \\
\hline O II & 4650.00 & - & - & 124 & 8.49 & 130 & 8.60 & - & - & - & - \\
\hline O II & 4661.63 & 70 & 8.43 & 42 & 8.42 & - & - & 94 & 8.49 & - & - \\
\hline O II & 4673.73 & - & - & - & - & - & - & 42 & 8.77 & - & - \\
\hline O II & 4676.23 & - & - & 37 & 8.44 & - & - & 77 & 8.42 & - & - \\
\hline O II & 4699.00 & 81 & 8.36 & 42 & 8.37 & 38 & 8.31 & 107 & 8.45 & 107 & 8.43 \\
\hline O II & 4705.35 & 54 & 8.16 & 40 & 8.43 & - & - & 85 & 8.38 & 86 & 8.40 \\
\hline O II & 4710.00 & - & - & 20 & 8.96 & - & - & 46 & 8.88 & - & - \\
\hline Mg II & 4481.00 & 129 & 7.47 & 145 & 7.32 & - & - & 130 & 7.38 & - & - \\
\hline Si II & 4128.05 & - & - & 27 & 7.37 & - & - & - & - & - & - \\
\hline Si II & 4130.89 & - & - & 33 & 7.30 & - & - & - & - & - & - \\
\hline Si III & 4552.62 & 142 & 7.52 & 123 & 7.52 & 99 & 7.34 & 173 & 7.38 & 138 & 7.30 \\
\hline Si III & 4567.84 & 117 & 7.51 & 97 & 7.47 & 77 & 7.28 & 143 & 7.36 & 130 & 7.49 \\
\hline Si III & 4574.75 & 71 & 7.38 & 58 & 7.40 & 46 & 7.23 & 107 & 7.54 & 94 & 7.61 \\
\hline Si IV & 4088.90 & - & - & - & - & - & - & - & - & - & - \\
\hline Si IV & 4116.10 & - & - & - & - & - & - & 64 & 7.53 & - & - \\
\hline Si IV & 4212.00 & - & - & - & - & - & - & - & - & - & - \\
\hline
\end{tabular}


I. Hunter et al.: Chemical compositions of B-type stars, Online Material p 5

Table 1. continued.

\begin{tabular}{|c|c|c|c|c|c|c|c|c|c|c|c|}
\hline \multirow[t]{2}{*}{ Species } & \multirow[t]{2}{*}{ Wavelength } & \multicolumn{2}{|c|}{ NGC 6611-042 } & \multicolumn{2}{|c|}{ NGC 6611-052 } & \multicolumn{2}{|c|}{ NGC 6611-062 } & \multicolumn{2}{|c|}{ NGC 6611-063 } & \multicolumn{2}{|c|}{ NGC 6611-066 } \\
\hline & & $E W$ & Abund & $E W$ & Abund & $E W$ & Abund & $E W$ & Abund & $E W$ & Abund \\
\hline C II & 4267.00 & 184 & 8.03 & 180 & 7.74 & 181 & 7.91 & 201 & 8.19 & 201 & 8.18 \\
\hline N II & 3955.85 & - & - & - & - & - & - & - & - & - & - \\
\hline N II & 3995.00 & 79 & 7.43 & 66 & 7.62 & 36 & 7.48 & 63 & 7.33 & 41 & 7.36 \\
\hline N II & 4447.03 & 47 & 7.57 & - & - & - & - & - & - & - & - \\
\hline N II & 4601.48 & 61 & 7.98 & - & - & - & - & - & - & - & - \\
\hline N II & 4613.86 & - & - & - & - & - & - & - & - & - & - \\
\hline N II & 4630.54 & 79 & 7.69 & 58 & 7.83 & - & - & 77 & 7.81 & 47 & 7.83 \\
\hline N II & 4643.08 & - & - & - & - & - & - & - & - & - & - \\
\hline O II & 3912.00 & 65 & 8.67 & - & - & - & - & - & - & - & - \\
\hline O II & 3919.30 & - & - & - & - & - & - & - & - & - & - \\
\hline O II & 3945.04 & - & - & - & - & - & - & - & - & - & - \\
\hline O II & 3954.36 & 54 & 8.52 & - & - & - & - & - & - & - & - \\
\hline O II & 3982.71 & - & - & - & - & - & - & - & - & - & - \\
\hline O II & 4069.00 & - & - & - & - & - & - & - & - & - & - \\
\hline O II & 4072.15 & - & - & - & - & - & - & - & - & - & - \\
\hline O II & 4075.86 & - & - & - & - & - & - & - & - & - & - \\
\hline O II & 4078.84 & - & - & - & - & - & - & - & - & - & - \\
\hline O II & 4132.80 & - & - & - & - & - & - & - & - & - & - \\
\hline O II & 4156.53 & - & - & - & - & - & - & - & - & - & - \\
\hline O II & 4185.44 & - & - & - & - & - & - & - & - & - & - \\
\hline O II & 4317.00 & 68 & 8.74 & 44 & 8.95 & - & - & - & - & - & - \\
\hline O II & 4319.63 & 64 & 8.68 & 36 & 8.80 & - & - & - & - & - & - \\
\hline O II & 4325.76 & - & - & - & - & - & - & - & - & - & - \\
\hline O II & 4349.43 & - & - & - & - & - & - & - & - & - & - \\
\hline O II & 4351.00 & - & - & - & - & - & - & - & - & - & - \\
\hline O II & 4353.58 & - & - & - & - & - & - & - & - & - & - \\
\hline O II & 4366.00 & - & - & - & - & - & - & - & - & - & - \\
\hline O II & 4369.27 & - & - & - & - & - & - & - & - & - & - \\
\hline O II & 4395.94 & - & - & - & - & - & - & - & - & - & - \\
\hline O II & 4414.90 & 69 & 8.23 & 56 & 8.66 & - & - & - & - & 55 & 8.78 \\
\hline O II & 4416.98 & 48 & 8.19 & 39 & 8.63 & - & - & - & - & 40 & 8.78 \\
\hline O II & 4443.01 & - & - & - & - & - & - & - & - & - & - \\
\hline O II & 4452.38 & - & - & - & - & - & - & - & - & - & - \\
\hline O II & 4590.97 & 78 & 8.75 & 42 & 8.90 & - & - & - & - & - & - \\
\hline O II & 4596.18 & 69 & 8.67 & - & - & - & - & - & - & - & - \\
\hline O II & 4638.86 & 71 & 8.70 & 45 & 8.95 & - & - & - & - & - & - \\
\hline O II & 4641.81 & 135 & 9.01 & 62 & 8.79 & - & - & - & - & - & - \\
\hline O II & 4650.00 & 244 & 8.94 & 105 & 8.70 & 65 & 8.73 & 189 & 8.82 & - & - \\
\hline O II & 4661.63 & 63 & 8.51 & - & - & - & - & - & - & - & - \\
\hline O II & 4673.73 & - & - & - & - & - & - & - & - & - & - \\
\hline O II & 4676.23 & - & - & - & - & - & - & - & - & - & - \\
\hline O II & 4699.00 & 76 & 8.63 & 44 & 8.99 & - & - & - & - & - & - \\
\hline O II & 4705.35 & 46 & 8.30 & 39 & 8.92 & - & - & - & - & - & - \\
\hline O II & 4710.00 & - & - & - & - & - & - & - & - & - & - \\
\hline Mg II & 4481.00 & 196 & 7.57 & 159 & 6.86 & 209 & 7.04 & 151 & 7.34 & 175 & 7.43 \\
\hline Si II & 4128.05 & - & - & - & - & - & - & - & - & - & - \\
\hline Si II & 4130.89 & - & - & - & - & - & - & - & - & - & - \\
\hline Si III & 4552.62 & 181 & 7.67 & 99 & 7.25 & 78 & 7.42 & 138 & 7.51 & 75 & 7.37 \\
\hline Si III & 4567.84 & 132 & 7.48 & 88 & 7.38 & - & - & 97 & 7.30 & 57 & 7.31 \\
\hline Si III & 4574.75 & 72 & 7.28 & 68 & 7.64 & - & - & 83 & 7.65 & - & - \\
\hline Si IV & 4088.90 & - & - & - & - & - & - & - & - & - & - \\
\hline Si IV & 4116.10 & - & - & - & - & - & - & - & - & - & - \\
\hline Si IV & 4212.00 & - & - & - & - & - & - & - & - & - & - \\
\hline
\end{tabular}


I. Hunter et al.: Chemical compositions of B-type stars, Online Material p 6

Table 1. continued.

\begin{tabular}{|c|c|c|c|c|c|c|c|c|c|c|c|}
\hline \multirow[t]{2}{*}{ Species } & \multirow[t]{2}{*}{ Wavelength } & \multicolumn{2}{|c|}{ NGC 3293-001 } & \multicolumn{2}{|c|}{ NGC 3293-002 } & \multicolumn{2}{|c|}{ NGC 3293-003 } & \multicolumn{2}{|c|}{ NGC 3293-004 } & \multicolumn{2}{|c|}{ NGC 3293-005 } \\
\hline & & $E W$ & Abund & $E W$ & Abund & $E W$ & Abund & $E W$ & Abund & $E W$ & Abund \\
\hline C II & 4267.00 & 98 & 8.01 & 150 & 8.10 & 237 & 7.95 & 214 & 8.17 & 203 & 7.89 \\
\hline N II & 3955.85 & - & - & 46 & 8.10 & - & - & - & - & - & - \\
\hline N II & 3995.00 & 97 & 7.83 & 188 & 8.01 & 127 & 7.41 & 115 & 7.39 & 152 & 7.50 \\
\hline N II & 4447.03 & 58 & 7.84 & - & - & 65 & 7.52 & 78 & 7.63 & - & - \\
\hline N II & 4601.48 & 36 & 7.84 & 111 & 8.22 & 68 & 7.64 & 69 & 7.71 & 69 & 7.62 \\
\hline $\mathrm{N}$ II & 4613.86 & 18 & 7.73 & 54 & 8.00 & 37 & 7.55 & 35 & 7.58 & - & - \\
\hline N II & 4630.54 & 125 & 8.01 & 183 & 8.05 & 115 & 7.50 & 94 & 7.43 & 136 & 7.57 \\
\hline N II & 4643.08 & - & - & - & - & - & - & - & - & - & - \\
\hline O II & 3912.00 & 81 & 8.49 & 130 & 8.61 & 94 & 8.48 & - & - & 135 & 8.69 \\
\hline O II & 3919.30 & - & - & - & - & - & - & - & - & - & - \\
\hline O II & 3945.04 & 33 & 8.32 & 79 & 8.53 & 78 & 8.56 & 105 & 8.67 & 108 & 8.75 \\
\hline O II & 3954.36 & 70 & 8.37 & 158 & 8.65 & 118 & 8.53 & - & - & 120 & 8.49 \\
\hline O II & 3982.71 & - & - & - & - & 106 & 8.77 & - & - & - & - \\
\hline O II & 4069.00 & - & - & 298 & 8.80 & 242 & 8.73 & 361 & 9.12 & - & - \\
\hline O II & 4072.15 & - & - & 250 & 8.80 & 209 & 8.81 & 208 & 8.61 & - & - \\
\hline O II & 4075.86 & 172 & 8.30 & 266 & 8.67 & 261 & 8.93 & 313 & 9.03 & - & - \\
\hline O II & 4078.84 & 17 & 7.99 & 45 & 8.29 & 58 & 8.51 & - & - & - & - \\
\hline O II & 4132.80 & - & - & - & - & - & - & 89 & 8.54 & - & - \\
\hline O II & 4156.53 & - & - & - & - & 41 & 8.93 & - & - & - & - \\
\hline O II & 4185.44 & - & - & - & - & - & - & - & - & - & - \\
\hline O II & 4317.00 & - & - & - & - & 182 & 9.04 & 188 & 8.91 & 178 & 8.93 \\
\hline O II & 4319.63 & - & - & - & - & 178 & 9.01 & 189 & 8.92 & 198 & 9.04 \\
\hline O II & 4325.76 & - & - & - & - & 39 & 8.59 & - & - & - & - \\
\hline O II & 4349.43 & - & - & - & - & - & - & - & - & - & - \\
\hline O II & 4351.00 & - & - & - & - & - & - & - & - & - & - \\
\hline O II & 4353.58 & - & - & - & - & - & - & - & - & - & - \\
\hline O II & 4366.00 & - & - & - & - & 180 & 8.93 & 222 & 9.01 & - & - \\
\hline O II & 4369.27 & - & - & - & - & 30 & 8.42 & - & - & - & - \\
\hline O II & 4395.94 & - & - & - & - & 72 & 8.76 & 67 & 8.59 & - & - \\
\hline O II & 4414.90 & 133 & 8.43 & 275 & 8.73 & 267 & 8.92 & 274 & 8.77 & 203 & 8.53 \\
\hline O II & 4416.98 & 97 & 8.48 & 230 & 8.81 & 194 & 8.81 & 196 & 8.66 & 264 & 9.08 \\
\hline O II & 4443.01 & - & - & - & - & - & - & - & - & - & - \\
\hline O II & 4452.38 & - & - & - & - & 81 & 8.83 & 79 & 8.70 & - & - \\
\hline O II & 4590.97 & 117 & 8.37 & 195 & 8.56 & 179 & 8.75 & 201 & 8.68 & 196 & 8.78 \\
\hline O II & 4596.18 & 88 & 8.33 & - & - & 148 & 8.67 & 173 & 8.64 & 158 & 8.67 \\
\hline O II & 4638.86 & 104 & 8.71 & 200 & 8.82 & 195 & 8.94 & 206 & 8.83 & - & - \\
\hline O II & 4641.81 & - & - & - & - & - & - & 395 & 9.21 & - & - \\
\hline O II & 4650.00 & - & - & - & - & - & - & - & - & - & - \\
\hline O II & 4661.63 & 124 & 8.73 & 235 & 8.87 & 206 & 8.91 & 233 & 8.88 & 209 & 8.85 \\
\hline O II & 4673.73 & 35 & 8.90 & 54 & 8.79 & 57 & 8.81 & - & - & - & - \\
\hline O II & 4676.23 & 140 & 8.90 & 226 & 8.96 & 195 & 8.99 & - & - & - & - \\
\hline O II & 4699.00 & 50 & 8.09 & 117 & 8.31 & 105 & 8.38 & 97 & 8.19 & 138 & 8.55 \\
\hline O II & 4705.35 & 58 & 8.13 & 147 & 8.47 & 120 & 8.50 & 134 & 8.41 & 156 & 8.66 \\
\hline O II & 4710.00 & - & - & - & - & 49 & 8.79 & 40 & 8.55 & - & - \\
\hline Mg II & 4481.00 & 109 & 7.33 & 176 & 7.56 & 191 & 7.29 & 198 & 7.44 & 238 & 7.47 \\
\hline Si II & 4128.05 & - & - & - & - & - & - & - & - & - & - \\
\hline Si II & 4130.89 & - & - & - & - & - & - & - & - & - & - \\
\hline Si III & 4552.62 & 299 & 7.41 & 431 & 7.44 & 393 & 7.53 & 390 & 7.44 & 398 & 7.46 \\
\hline Si III & 4567.84 & 245 & 7.47 & 358 & 7.45 & 321 & 7.48 & 330 & 7.46 & 332 & 7.46 \\
\hline Si III & 4574.75 & 129 & 7.53 & 211 & 7.44 & 199 & 7.44 & 206 & 7.44 & 217 & 7.50 \\
\hline Si IV & 4088.90 & - & - & - & - & - & - & - & - & - & - \\
\hline Si IV & 4116.10 & 467 & 7.47 & 236 & 7.47 & 91 & 7.49 & 121 & 7.45 & - & - \\
\hline Si IV & 4212.00 & - & - & - & - & - & - & - & - & - & - \\
\hline
\end{tabular}


I. Hunter et al.: Chemical compositions of B-type stars, Online Material p 7

Table 1. continued.

\begin{tabular}{|c|c|c|c|c|c|c|c|c|c|c|c|}
\hline \multirow[t]{2}{*}{ Species } & \multirow[t]{2}{*}{ Wavelength } & \multicolumn{2}{|c|}{ NGC 3293-006 } & \multicolumn{2}{|c|}{ NGC 3293-007 } & \multicolumn{2}{|c|}{ NGC 3293-008 } & \multicolumn{2}{|c|}{ NGC 3293-010 } & \multicolumn{2}{|c|}{ NGC 3293-012 } \\
\hline & & $E W$ & Abund & $E W$ & Abund & $E W$ & Abund & $E W$ & Abund & $E W$ & Abund \\
\hline C II & 4267.00 & 193 & 7.81 & 200 & 8.13 & 195 & 7.83 & 194 & 7.85 & 201 & 7.86 \\
\hline N II & 3955.85 & - & - & 27 & 7.56 & - & - & 27 & 7.51 & - & - \\
\hline N II & 3995.00 & 115 & 7.29 & 123 & 7.48 & 108 & 7.30 & 116 & 7.37 & 94 & 7.23 \\
\hline N II & 4447.03 & - & - & 59 & 7.49 & 89 & 7.70 & 49 & 7.34 & - & - \\
\hline N II & 4601.48 & - & - & - & - & 77 & 7.75 & 39 & 7.37 & 40 & 7.40 \\
\hline N II & 4613.86 & - & - & - & - & 55 & 7.78 & 46 & 7.67 & 47 & 7.71 \\
\hline N II & 4630.54 & 108 & 7.45 & 101 & 7.49 & 98 & 7.43 & 95 & 7.42 & 101 & 7.48 \\
\hline N II & 4643.08 & - & - & - & - & - & - & - & - & - & - \\
\hline O II & 3912.00 & 96 & 8.45 & 140 & 8.69 & 116 & 8.71 & 124 & 8.78 & 103 & 8.66 \\
\hline O II & 3919.30 & - & - & - & - & - & - & - & - & - & - \\
\hline O II & 3945.04 & 113 & 8.81 & 92 & 8.61 & 80 & 8.67 & 77 & 8.65 & 88 & 8.80 \\
\hline O II & 3954.36 & 140 & 8.65 & 121 & 8.48 & 111 & 8.60 & 90 & 8.43 & 99 & 8.56 \\
\hline O II & 3982.71 & - & - & 89 & 8.58 & - & - & - & - & 92 & 8.85 \\
\hline O II & 4069.00 & - & - & 271 & 8.78 & 238 & 8.84 & 245 & 8.90 & 254 & 9.01 \\
\hline O II & 4072.15 & - & - & 220 & 8.83 & 207 & 8.95 & 196 & 8.92 & 187 & 8.91 \\
\hline O II & 4075.86 & - & - & 243 & 8.78 & 220 & 8.84 & 203 & 8.78 & 230 & 9.02 \\
\hline O II & 4078.84 & - & - & 55 & 8.38 & 44 & 8.38 & 59 & 8.60 & 53 & 8.57 \\
\hline O II & 4132.80 & - & - & 87 & 8.56 & - & - & 92 & 8.77 & - & - \\
\hline O II & 4156.53 & - & - & - & - & - & - & 35 & 8.86 & - & - \\
\hline O II & 4185.44 & - & - & - & - & - & - & - & - & - & - \\
\hline O II & 4317.00 & 214 & 9.16 & 176 & 8.94 & 150 & 8.95 & 164 & 9.08 & 142 & 8.97 \\
\hline O II & 4319.63 & 189 & 9.01 & 184 & 9.00 & 163 & 9.04 & 165 & 9.09 & 137 & 8.93 \\
\hline O II & 4325.76 & - & - & 64 & 8.84 & - & - & 51 & 8.83 & - & - \\
\hline O II & 4349.43 & - & - & - & - & - & - & - & - & - & - \\
\hline O II & 4351.00 & - & - & - & - & - & - & - & - & - & - \\
\hline O II & 4353.58 & - & - & - & - & - & - & - & - & - & - \\
\hline O II & 4366.00 & 213 & 9.06 & 191 & 8.94 & - & - & 164 & 8.98 & 147 & 8.91 \\
\hline O II & 4369.27 & - & - & 40 & 8.49 & - & - & 45 & 8.71 & - & - \\
\hline O II & 4395.94 & - & - & 70 & 8.64 & 59 & 8.70 & 78 & 8.90 & 72 & 8.89 \\
\hline O II & 4414.90 & 252 & 8.79 & 253 & 8.78 & 178 & 8.61 & 215 & 8.85 & 168 & 8.62 \\
\hline O II & 4416.98 & 245 & 9.02 & 190 & 8.71 & 162 & 8.77 & 167 & 8.82 & 135 & 8.66 \\
\hline O II & 4443.01 & - & - & - & - & - & - & - & - & - & - \\
\hline O II & 4452.38 & - & - & 82 & 8.76 & - & - & 83 & 8.96 & 71 & 8.88 \\
\hline O II & 4590.97 & 215 & 8.91 & 186 & 8.69 & 163 & 8.83 & 154 & 8.79 & 149 & 8.82 \\
\hline O II & 4596.18 & 153 & 8.69 & 161 & 8.64 & 141 & 8.78 & 146 & 8.82 & 146 & 8.89 \\
\hline O II & 4638.86 & - & - & 193 & 8.85 & 168 & 8.94 & 172 & 8.98 & 152 & 8.91 \\
\hline O II & 4641.81 & - & - & 296 & 8.90 & 293 & 9.15 & 212 & 8.75 & 268 & 9.13 \\
\hline O II & 4650.00 & - & - & - & - & - & - & - & - & - & - \\
\hline O II & 4661.63 & 216 & 8.92 & 206 & 8.83 & 174 & 8.89 & 88 & 8.28 & 159 & 8.87 \\
\hline O II & 4673.73 & - & - & 65 & 8.82 & - & - & 63 & 8.98 & 56 & 8.94 \\
\hline O II & 4676.23 & - & - & 138 & 8.58 & - & - & 137 & 8.80 & 145 & 8.92 \\
\hline O II & 4699.00 & 157 & 8.69 & 135 & 8.44 & 103 & 8.49 & 130 & 8.67 & 106 & 8.56 \\
\hline O II & 4705.35 & 176 & 8.80 & 148 & 8.55 & 100 & 8.48 & 126 & 8.68 & 112 & 8.64 \\
\hline O II & 4710.00 & - & - & 61 & 8.83 & - & - & 71 & 9.18 & 48 & 8.95 \\
\hline Mg II & 4481.00 & 181 & 7.19 & 152 & 7.26 & 180 & 7.20 & 168 & 7.17 & 199 & 7.29 \\
\hline Si II & 4128.05 & - & - & 24 & 7.45 & - & - & 24 & 7.03 & - & - \\
\hline Si II & 4130.89 & - & - & 27 & 7.31 & - & - & 25 & 6.86 & - & - \\
\hline Si III & 4552.62 & 408 & 7.50 & 350 & 7.44 & 328 & 7.47 & 313 & 7.46 & 306 & 7.48 \\
\hline Si III & 4567.84 & 326 & 7.44 & 300 & 7.48 & 269 & 7.45 & 269 & 7.50 & 256 & 7.49 \\
\hline Si III & 4574.75 & 201 & 7.43 & 184 & 7.41 & 173 & 7.47 & 174 & 7.50 & 172 & 7.54 \\
\hline Si IV & 4088.90 & - & - & - & - & - & - & - & - & - & - \\
\hline Si IV & 4116.10 & - & - & 110 & 7.44 & - & - & 64 & 7.53 & 54 & 7.51 \\
\hline Si IV & 4212.00 & - & - & - & - & - & - & - & - & - & - \\
\hline
\end{tabular}


I. Hunter et al.: Chemical compositions of B-type stars, Online Material p 8

Table 1. continued.

\begin{tabular}{|c|c|c|c|c|c|c|c|c|c|c|c|}
\hline \multirow[t]{2}{*}{ Species } & \multirow[t]{2}{*}{ Wavelength } & \multicolumn{2}{|c|}{ NGC 3293-016 } & \multicolumn{2}{|c|}{ NGC 3293-018 } & \multicolumn{2}{|c|}{ NGC 3293-019 } & \multicolumn{2}{|c|}{ NGC 3293-023 } & \multicolumn{2}{|c|}{ NGC 3293-024 } \\
\hline & & $E W$ & Abund & $E W$ & Abund & $E W$ & Abund & $E W$ & Abund & $E W$ & Abund \\
\hline C II & 4267.00 & 199 & 8.13 & 155 & 7.96 & 180 & 8.26 & 178 & 7.72 & 182 & 7.75 \\
\hline N II & 3955.85 & - & - & 22 & 7.57 & - & - & - & - & - & - \\
\hline N II & 3995.00 & 35 & 7.38 & 94 & 7.47 & - & - & 72 & 7.30 & 71 & 7.33 \\
\hline N II & 4447.03 & 25 & 7.81 & 50 & 7.50 & - & - & 56 & 7.69 & 48 & 7.63 \\
\hline N II & 4601.48 & - & - & - & - & - & - & 40 & 7.61 & 55 & 7.90 \\
\hline N II & 4613.86 & - & - & - & - & - & - & - & - & - & - \\
\hline N II & 4630.54 & 36 & 7.78 & 83 & 7.58 & 83 & 7.59 & 81 & 7.66 & 74 & 7.63 \\
\hline N II & 4643.08 & - & - & 51 & 7.69 & - & - & - & - & - & - \\
\hline O II & 3912.00 & - & - & 94 & 8.72 & - & - & 71 & 8.79 & 77 & 8.94 \\
\hline O II & 3919.30 & - & - & - & - & - & - & - & - & - & - \\
\hline O II & 3945.04 & - & - & 55 & 8.58 & - & - & 49 & 8.77 & 53 & 8.91 \\
\hline O II & 3954.36 & - & - & 82 & 8.60 & - & - & 57 & 8.58 & 63 & 8.75 \\
\hline O II & 3982.71 & - & - & 56 & 8.63 & - & - & - & - & - & - \\
\hline O II & 4069.00 & - & - & 185 & 8.81 & - & - & - & - & 134 & 8.87 \\
\hline O II & 4072.15 & - & - & 105 & 8.54 & - & - & - & - & 95 & 8.86 \\
\hline O II & 4075.86 & - & - & 131 & 8.67 & - & - & - & - & 131 & 9.11 \\
\hline O II & 4078.84 & - & - & 43 & 8.53 & - & - & - & - & 19 & 8.29 \\
\hline O II & 4132.80 & - & - & 54 & 8.50 & - & - & - & - & - & - \\
\hline O II & 4156.53 & - & - & - & - & - & - & - & - & - & - \\
\hline O II & 4185.44 & - & - & - & - & - & - & - & - & - & - \\
\hline O II & 4317.00 & - & - & 96 & 8.83 & - & - & 82 & 8.96 & 69 & 8.85 \\
\hline O II & 4319.63 & - & - & 102 & 8.91 & - & - & 56 & 8.58 & 58 & 8.68 \\
\hline O II & 4325.76 & - & - & - & - & - & - & - & - & - & - \\
\hline O II & 4349.43 & - & - & - & - & - & - & - & - & - & - \\
\hline O II & 4351.00 & - & - & - & - & - & - & - & - & - & - \\
\hline O II & 4353.58 & - & - & - & - & - & - & - & - & - & - \\
\hline O II & 4366.00 & - & - & 100 & 8.78 & - & - & - & - & - & - \\
\hline O II & 4369.27 & - & - & 29 & 8.53 & - & - & - & - & - & - \\
\hline O II & 4395.94 & - & - & 60 & 8.88 & - & - & - & - & - & - \\
\hline O II & 4414.90 & - & - & 140 & 8.70 & 138 & 8.41 & 65 & 8.22 & 68 & 8.34 \\
\hline O II & 4416.98 & - & - & 116 & 8.77 & 146 & 8.78 & 84 & 8.76 & 78 & 8.76 \\
\hline O II & 4443.01 & - & - & - & - & - & - & - & - & - & - \\
\hline O II & 4452.38 & - & - & 58 & 8.85 & - & - & - & - & - & - \\
\hline O II & 4590.97 & - & - & 100 & 8.65 & 138 & 8.74 & 62 & 8.58 & 71 & 8.80 \\
\hline O II & 4596.18 & - & - & 104 & 8.73 & 151 & 8.89 & 49 & 8.45 & 64 & 8.76 \\
\hline O II & 4638.86 & - & - & 112 & 8.86 & 122 & 8.69 & 72 & 8.75 & 86 & 9.02 \\
\hline O II & 4641.81 & - & - & 142 & 8.69 & 156 & 8.51 & 139 & 9.04 & 115 & 8.89 \\
\hline O II & 4650.00 & 59 & 8.58 & - & - & - & - & 268 & 9.07 & 249 & 9.06 \\
\hline O II & 4661.63 & - & - & 111 & 8.76 & - & - & 68 & 8.62 & 64 & 8.65 \\
\hline O II & 4673.73 & - & - & 40 & 8.80 & - & - & - & - & - & - \\
\hline O II & 4676.23 & - & - & 92 & 8.69 & - & - & - & - & - & - \\
\hline O II & 4699.00 & - & - & 108 & 8.62 & 104 & 8.35 & 77 & 8.77 & 56 & 8.58 \\
\hline O II & 4705.35 & - & - & 86 & 8.54 & 83 & 8.25 & 42 & 8.34 & - & - \\
\hline O II & 4710.00 & - & - & 49 & 9.07 & - & - & - & - & - & - \\
\hline Mg II & 4481.00 & 191 & 7.35 & 126 & 7.15 & 162 & 7.51 & 149 & 7.04 & 156 & 7.06 \\
\hline Si II & 4128.05 & - & - & 19 & 7.18 & - & - & - & - & - & - \\
\hline Si II & 4130.89 & - & - & 12 & 6.69 & - & - & - & - & - & - \\
\hline Si III & 4552.62 & 75 & 7.55 & 192 & 7.46 & 220 & 7.45 & 171 & 7.45 & 162 & 7.45 \\
\hline Si III & 4567.84 & 49 & 7.34 & 164 & 7.49 & 176 & 7.38 & 135 & 7.39 & 124 & 7.36 \\
\hline Si III & 4574.75 & 32 & 7.44 & 113 & 7.53 & 115 & 7.39 & 90 & 7.45 & 83 & 7.43 \\
\hline Si IV & 4088.90 & - & - & - & - & - & - & - & - & - & - \\
\hline Si IV & 4116.10 & - & - & 44 & 7.49 & - & - & - & - & - & - \\
\hline Si IV & 4212.00 & - & - & - & - & - & - & - & - & - & - \\
\hline
\end{tabular}


I. Hunter et al.: Chemical compositions of B-type stars, Online Material p 9

Table 1. continued.

\begin{tabular}{|c|c|c|c|c|c|c|c|c|c|c|c|}
\hline \multirow[t]{2}{*}{ Species } & \multirow[t]{2}{*}{ Wavelength } & \multicolumn{2}{|c|}{ NGC 3293-025 } & \multicolumn{2}{|c|}{ NGC 3293-026 } & \multicolumn{2}{|c|}{ NGC 3293-028 } & \multicolumn{2}{|c|}{ NGC 3293-030 } & \multicolumn{2}{|c|}{ NGC 3293-031 } \\
\hline & & $E W$ & Abund & $E W$ & Abund & $E W$ & Abund & $E W$ & Abund & $E W$ & Abund \\
\hline C II & 4267.00 & 179 & 7.84 & 181 & 8.03 & 178 & 7.69 & 189 & 7.86 & 189 & 7.66 \\
\hline N II & 3955.85 & - & - & 26 & 7.72 & - & - & - & - & - & - \\
\hline N II & 3995.00 & - & - & 89 & 7.58 & 50 & 7.28 & 62 & 7.51 & - & - \\
\hline N II & 4447.03 & - & - & 55 & 7.69 & 29 & 7.54 & 35 & 7.68 & - & - \\
\hline N II & 4601.48 & - & - & 42 & 7.67 & - & - & 18 & 7.40 & - & - \\
\hline N II & 4613.86 & - & - & - & - & - & - & - & - & - & - \\
\hline N II & 4630.54 & 53 & 7.41 & - & - & 62 & 7.75 & 55 & 7.71 & 43 & 7.70 \\
\hline N II & 4643.08 & - & - & - & - & - & - & - & - & - & - \\
\hline O II & 3912.00 & - & - & 71 & 8.73 & - & - & - & - & - & - \\
\hline O II & 3919.30 & - & - & - & - & - & - & - & - & - & - \\
\hline O II & 3945.04 & - & - & 45 & 8.66 & - & - & - & - & - & - \\
\hline O II & 3954.36 & - & - & 66 & 8.70 & - & - & - & - & - & - \\
\hline O II & 3982.71 & - & - & 45 & 8.72 & - & - & - & - & - & - \\
\hline O II & 4069.00 & - & - & 131 & 8.73 & - & - & - & - & - & - \\
\hline O II & 4072.15 & - & - & 83 & 8.65 & - & - & - & - & - & - \\
\hline O II & 4075.86 & - & - & 113 & 8.91 & - & - & - & - & - & - \\
\hline O II & 4078.84 & - & - & 39 & 8.73 & - & - & - & - & - & - \\
\hline O II & 4132.80 & - & - & - & - & - & - & - & - & - & - \\
\hline O II & 4156.53 & - & - & 46 & 9.42 & - & - & - & - & - & - \\
\hline O II & 4185.44 & - & - & - & - & - & - & - & - & - & - \\
\hline O II & 4317.00 & - & - & 76 & 8.90 & - & - & 36 & 8.66 & - & - \\
\hline O II & 4319.63 & - & - & 71 & 8.81 & - & - & 29 & 8.49 & - & - \\
\hline O II & 4325.76 & - & - & 32 & 8.89 & - & - & - & - & - & - \\
\hline O II & 4349.43 & - & - & - & - & - & - & - & - & - & - \\
\hline O II & 4351.00 & - & - & - & - & - & - & - & - & - & - \\
\hline O II & 4353.58 & - & - & - & - & - & - & - & - & - & - \\
\hline O II & 4366.00 & - & - & 75 & 8.81 & - & - & - & - & - & - \\
\hline O II & 4369.27 & - & - & 22 & 8.56 & - & - & - & - & - & - \\
\hline O II & 4395.94 & - & - & 50 & 9.03 & - & - & - & - & - & - \\
\hline O II & 4414.90 & - & - & 104 & 8.71 & 43 & 8.28 & - & - & - & - \\
\hline O II & 4416.98 & - & - & 89 & 8.84 & 60 & 8.85 & - & - & - & - \\
\hline O II & 4443.01 & - & - & - & - & - & - & - & - & - & - \\
\hline O II & 4452.38 & - & - & 42 & 8.84 & - & - & - & - & - & - \\
\hline O II & 4590.97 & - & - & 73 & 8.69 & - & - & - & - & - & - \\
\hline O II & 4596.18 & - & - & 76 & 8.75 & - & - & - & - & - & - \\
\hline O II & 4638.86 & 91 & 9.14 & 78 & 8.82 & - & - & - & - & - & - \\
\hline O II & 4641.81 & 62 & 8.29 & 100 & 8.67 & - & - & - & - & - & - \\
\hline O II & 4650.00 & 168 & 8.66 & 117 & 8.11 & 165 & 8.96 & 148 & 8.95 & - & - \\
\hline O II & 4661.63 & - & - & 77 & 8.73 & 43 & 8.68 & 33 & 8.51 & - & - \\
\hline O II & 4673.73 & - & - & 30 & 8.83 & - & - & - & - & - & - \\
\hline O II & 4676.23 & - & - & 67 & 8.71 & - & - & - & - & - & - \\
\hline O II & 4699.00 & - & - & 79 & 8.64 & - & - & - & - & - & - \\
\hline O II & 4705.35 & - & - & 66 & 8.63 & - & - & - & - & - & - \\
\hline O II & 4710.00 & - & - & 38 & 9.18 & - & - & - & - & - & - \\
\hline Mg II & 4481.00 & 134 & 6.99 & 135 & 7.22 & 165 & 6.96 & 148 & 6.99 & - & - \\
\hline Si II & 4128.05 & - & - & 22 & 7.13 & - & - & - & - & - & - \\
\hline Si II & 4130.89 & - & - & 21 & 6.88 & - & - & - & - & - & - \\
\hline Si III & 4552.62 & 152 & 7.48 & 141 & 7.43 & 143 & 7.56 & 120 & 7.55 & 123 & 7.48 \\
\hline Si III & 4567.84 & 116 & 7.38 & 116 & 7.42 & 92 & 7.30 & 83 & 7.36 & 83 & 7.36 \\
\hline Si III & 4574.75 & 80 & 7.47 & 85 & 7.56 & 59 & 7.39 & 52 & 7.39 & 57 & 7.55 \\
\hline Si IV & 4088.90 & - & - & - & - & - & - & - & - & - & - \\
\hline Si IV & 4116.10 & - & - & 24 & 7.46 & - & - & - & - & - & - \\
\hline Si IV & 4212.00 & - & - & - & - & - & - & - & - & - & - \\
\hline
\end{tabular}


I. Hunter et al.: Chemical compositions of B-type stars, Online Material $p 10$

Table 1. continued.

\begin{tabular}{|c|c|c|c|c|c|c|c|c|c|c|c|}
\hline \multirow[t]{2}{*}{ Species } & \multirow[t]{2}{*}{ Wavelength } & \multicolumn{2}{|c|}{ NGC 3293-034 } & \multicolumn{2}{|c|}{ NGC 3293-035 } & \multicolumn{2}{|c|}{ NGC 3293-037 } & \multicolumn{2}{|c|}{ NGC 3293-038 } & \multicolumn{2}{|c|}{ NGC 3293-041 } \\
\hline & & $E W$ & Abund & $E W$ & Abund & $E W$ & Abund & $E W$ & Abund & $E W$ & Abund \\
\hline C II & 4267.00 & 157 & 8.28 & 196 & 7.92 & 194 & 7.99 & 211 & 8.16 & 230 & 8.34 \\
\hline N II & 3955.85 & 17 & 7.66 & - & - & - & - & - & - & - & - \\
\hline N II & 3995.00 & 64 & 7.41 & - & - & 70 & 7.62 & 53 & 7.46 & 40 & 7.26 \\
\hline N II & 4447.03 & 48 & 7.66 & - & - & 35 & 7.65 & - & - & - & - \\
\hline N II & 4601.48 & 43 & 7.87 & - & - & 45 & 8.03 & - & - & - & - \\
\hline N II & 4613.86 & 22 & 7.64 & - & - & - & - & - & - & - & - \\
\hline N II & 4630.54 & 51 & 7.47 & 59 & 7.64 & 61 & 7.80 & 47 & 7.68 & 50 & 7.81 \\
\hline N II & 4643.08 & 27 & 7.50 & - & - & - & - & - & - & - & - \\
\hline O II & 3912.00 & 46 & 8.19 & - & - & 43 & 8.77 & - & - & - & - \\
\hline O II & 3919.30 & - & - & - & - & - & - & - & - & - & - \\
\hline O II & 3945.04 & 30 & 8.22 & - & - & - & - & - & - & - & - \\
\hline O II & 3954.36 & 38 & 8.07 & - & - & - & - & - & - & - & - \\
\hline O II & 3982.71 & - & - & - & - & - & - & - & - & - & - \\
\hline O II & 4069.00 & 87 & 8.03 & - & - & 89 & 8.79 & - & - & - & - \\
\hline O II & 4072.15 & 59 & 8.01 & - & - & 63 & 8.81 & - & - & - & - \\
\hline O II & 4075.86 & 83 & 8.24 & - & - & 86 & 9.01 & - & - & - & - \\
\hline O II & 4078.84 & 22 & 8.09 & - & - & - & - & - & - & - & - \\
\hline O II & 4132.80 & - & - & - & - & - & - & - & - & - & - \\
\hline O II & 4156.53 & - & - & - & - & - & - & - & - & - & - \\
\hline O II & 4185.44 & - & - & - & - & - & - & - & - & - & - \\
\hline O II & 4317.00 & 51 & 8.29 & - & - & 40 & 8.71 & - & - & - & - \\
\hline O II & 4319.63 & 48 & 8.23 & - & - & 36 & 8.62 & - & - & - & - \\
\hline O II & 4325.76 & - & - & - & - & - & - & - & - & - & - \\
\hline O II & 4349.43 & - & - & - & - & - & - & - & - & - & - \\
\hline O II & 4351.00 & - & - & - & - & - & - & - & - & - & - \\
\hline O II & 4353.58 & - & - & - & - & - & - & - & - & - & - \\
\hline O II & 4366.00 & 45 & 8.13 & - & - & 35 & 8.53 & - & - & - & - \\
\hline O II & 4369.27 & 11 & 7.97 & - & - & - & - & - & - & - & - \\
\hline O II & 4395.94 & 23 & 8.22 & - & - & - & - & - & - & - & - \\
\hline O II & 4414.90 & 75 & 8.14 & 51 & 8.30 & 67 & 8.69 & - & - & - & - \\
\hline O II & 4416.98 & 64 & 8.28 & 53 & 8.63 & 51 & 8.72 & - & - & - & - \\
\hline O II & 4443.01 & - & - & - & - & - & - & - & - & - & - \\
\hline O II & 4452.38 & - & - & - & - & - & - & - & - & - & - \\
\hline O II & 4590.97 & 46 & 7.97 & - & - & 41 & 8.68 & - & - & - & - \\
\hline O II & 4596.18 & 46 & 8.06 & - & - & 39 & 8.70 & - & - & - & - \\
\hline O II & 4638.86 & 54 & 8.24 & - & - & 53 & 8.93 & - & - & - & - \\
\hline O II & 4641.81 & 71 & 8.07 & - & - & 62 & 8.66 & - & - & - & - \\
\hline O II & 4650.00 & 160 & 8.27 & 140 & 8.68 & 140 & 8.83 & 97 & 8.61 & 99 & 8.71 \\
\hline O II & 4661.63 & 51 & 8.11 & - & - & 44 & 8.69 & - & - & - & - \\
\hline O II & 4673.73 & 16 & 8.26 & - & - & - & - & - & - & - & - \\
\hline O II & 4676.23 & 45 & 8.12 & - & - & 38 & 8.69 & - & - & - & - \\
\hline O II & 4699.00 & 49 & 7.96 & - & - & 39 & 8.60 & - & - & 29 & 8.53 \\
\hline O II & 4705.35 & 41 & 7.91 & - & - & 36 & 8.62 & - & - & - & - \\
\hline O II & 4710.00 & - & - & - & - & - & - & - & - & - & - \\
\hline Mg II & 4481.00 & 84 & 7.10 & 182 & 7.24 & 152 & 7.14 & 155 & 7.24 & 160 & 7.33 \\
\hline Si II & 4128.05 & - & - & - & - & - & - & - & - & - & - \\
\hline Si II & 4130.89 & - & - & - & - & - & - & - & - & - & - \\
\hline Si III & 4552.62 & 102 & 7.08 & 146 & 7.60 & 110 & 7.44 & 109 & 7.65 & - & - \\
\hline Si III & 4567.84 & 85 & 7.10 & 91 & 7.27 & 88 & 7.42 & 65 & 7.28 & 54 & 7.18 \\
\hline Si III & 4574.75 & 54 & 7.12 & 64 & 7.41 & 53 & 7.39 & 49 & 7.49 & - & - \\
\hline Si IV & 4088.90 & - & - & - & - & - & - & - & - & - & - \\
\hline Si IV & 4116.10 & 58 & 7.86 & - & - & - & - & - & - & - & - \\
\hline Si IV & 4212.00 & - & - & - & - & - & - & - & - & - & - \\
\hline
\end{tabular}


I. Hunter et al.: Chemical compositions of B-type stars, Online Material p 11

Table 1. continued.

\begin{tabular}{|c|c|c|c|c|c|c|c|c|c|c|c|}
\hline \multirow[t]{2}{*}{ Species } & \multirow[t]{2}{*}{ Wavelength } & \multicolumn{2}{|c|}{ NGC 3293-043 } & \multicolumn{2}{|c|}{ NGC 3293-062 } & \multicolumn{2}{|c|}{ NGC 4755-002 } & \multicolumn{2}{|c|}{ NGC 4755-003 } & \multicolumn{2}{|c|}{ NGC 4755-004 } \\
\hline & & $E W$ & Abund & $E W$ & Abund & $E W$ & Abund & $E W$ & Abund & $E W$ & Abund \\
\hline C II & 4267.00 & 169 & 7.94 & 162 & 8.07 & 289 & 7.72 & 274 & 7.70 & 240 & 7.79 \\
\hline N II & 3955.85 & 10 & 7.74 & - & - & 66 & 8.25 & 62 & 8.05 & 78 & 8.06 \\
\hline N II & 3995.00 & 34 & 7.35 & 28 & 7.64 & 192 & 8.06 & 219 & 8.04 & 269 & 7.99 \\
\hline N II & 4447.03 & 18 & 7.56 & - & - & 85 & 8.04 & 106 & 8.01 & 140 & 8.00 \\
\hline N II & 4601.48 & 11 & 7.42 & - & - & 103 & 8.24 & 118 & 8.18 & 161 & 8.20 \\
\hline N II & 4613.86 & 11 & 7.64 & - & - & 65 & 8.15 & 78 & 8.11 & 99 & 8.09 \\
\hline N II & 4630.54 & 25 & 7.49 & 24 & 7.92 & 189 & 8.27 & 211 & 8.21 & 264 & 8.11 \\
\hline N II & 4643.08 & 17 & 7.67 & - & - & 103 & 8.25 & 122 & 8.21 & 128 & 8.02 \\
\hline O II & 3912.00 & - & - & - & - & 46 & 8.67 & 52 & 8.45 & 105 & 8.59 \\
\hline O II & 3919.30 & - & - & - & - & - & - & - & - & - & - \\
\hline O II & 3945.04 & 13 & 8.64 & - & - & - & - & 38 & 8.45 & 71 & 8.52 \\
\hline O II & 3954.36 & 20 & 8.71 & - & - & 38 & 8.38 & 48 & 8.27 & 102 & 8.42 \\
\hline O II & 3982.71 & - & - & - & - & - & - & - & - & - & - \\
\hline O II & 4069.00 & 31 & 8.36 & - & - & 72 & 8.38 & 102 & 8.36 & 192 & 8.47 \\
\hline O II & 4072.15 & 25 & 8.47 & - & - & 84 & 8.61 & 113 & 8.61 & 204 & 8.70 \\
\hline O II & 4075.86 & 40 & 8.76 & - & - & 95 & 8.55 & 118 & 8.48 & 219 & 8.59 \\
\hline O II & 4078.84 & - & - & - & - & - & - & - & - & 37 & 8.24 \\
\hline O II & 4132.80 & - & - & - & - & - & - & 48 & 8.62 & 76 & 8.60 \\
\hline O II & 4156.53 & - & - & - & - & - & - & - & - & 24 & 8.68 \\
\hline O II & 4185.44 & - & - & - & - & - & - & - & - & - & - \\
\hline O II & 4317.00 & 18 & 8.55 & - & - & 59 & 8.74 & 75 & 8.65 & 154 & 8.83 \\
\hline O II & 4319.63 & 13 & 8.32 & - & - & 62 & 8.78 & 78 & 8.68 & 168 & 8.91 \\
\hline O II & 4325.76 & - & - & - & - & 15 & 8.60 & 20 & 8.52 & 41 & 8.64 \\
\hline O II & 4349.43 & - & - & - & - & 87 & 8.69 & - & - & 246 & 8.92 \\
\hline O II & 4351.00 & - & - & - & - & - & - & - & - & 93 & 8.35 \\
\hline O II & 4353.58 & - & - & - & - & - & - & - & - & - & - \\
\hline O II & 4366.00 & 15 & 8.38 & - & - & 55 & 8.60 & 78 & 8.61 & 161 & 8.78 \\
\hline O II & 4369.27 & - & - & - & - & 26 & 9.01 & - & - & - & - \\
\hline O II & 4395.94 & 14 & 9.03 & - & - & 49 & 9.26 & 51 & 8.97 & 65 & 8.74 \\
\hline O II & 4414.90 & 29 & 8.43 & - & - & 85 & 8.53 & 111 & 8.49 & 233 & 8.70 \\
\hline O II & 4416.98 & 23 & 8.55 & - & - & 61 & 8.52 & 86 & 8.53 & 169 & 8.64 \\
\hline O II & 4443.01 & - & - & - & - & - & - & - & - & - & - \\
\hline O II & 4452.38 & - & - & - & - & 19 & 8.53 & 29 & 8.54 & 66 & 8.72 \\
\hline O II & 4590.97 & 15 & 8.41 & - & - & 42 & 8.42 & 64 & 8.42 & 146 & 8.56 \\
\hline O II & 4596.18 & 13 & 8.41 & - & - & 44 & 8.57 & 59 & 8.47 & 123 & 8.53 \\
\hline O II & 4638.86 & 19 & 8.59 & - & - & 56 & 8.61 & 74 & 8.56 & 166 & 8.77 \\
\hline O II & 4641.81 & 24 & 8.36 & - & - & 101 & 8.63 & 121 & 8.53 & 295 & 8.89 \\
\hline O II & 4650.00 & - & - & 20 & 8.31 & 105 & 8.20 & 136 & 8.12 & 421 & 8.62 \\
\hline O II & 4661.63 & 17 & 8.44 & - & - & 44 & 8.37 & 84 & 8.58 & 184 & 8.78 \\
\hline O II & 4673.73 & - & - & - & - & 24 & 8.92 & 27 & 8.73 & 64 & 8.92 \\
\hline O II & 4676.23 & 15 & 8.47 & - & - & 54 & 8.64 & 73 & 8.61 & 161 & 8.79 \\
\hline O II & 4699.00 & 11 & 8.22 & - & - & 26 & 8.28 & 48 & 8.35 & 84 & 8.29 \\
\hline O II & 4705.35 & 14 & 8.41 & - & - & 36 & 8.46 & 48 & 8.34 & 108 & 8.44 \\
\hline O II & 4710.00 & - & - & - & - & - & - & 20 & 8.72 & 42 & 8.74 \\
\hline Mg II & 4481.00 & 151 & 7.14 & 182 & 7.11 & 350 & 7.30 & 294 & 7.33 & 215 & 7.29 \\
\hline Si II & 4128.05 & 35 & 7.42 & 57 & 7.83 & 141 & 7.54 & 107 & 7.53 & 61 & 7.50 \\
\hline Si II & 4130.89 & 41 & 7.31 & 49 & 7.18 & 149 & 7.39 & 118 & 7.40 & 73 & 7.41 \\
\hline Si III & 4552.62 & 60 & 7.34 & 48 & 7.54 & 241 & 7.41 & 281 & 7.42 & 415 & 7.38 \\
\hline Si III & 4567.84 & 43 & 7.24 & 30 & 7.35 & 192 & 7.41 & 224 & 7.40 & 343 & 7.38 \\
\hline Si III & 4574.75 & 28 & 7.34 & - & - & 108 & 7.39 & 132 & 7.38 & 208 & 7.38 \\
\hline Si IV & 4088.90 & - & - & - & - & - & - & - & - & - & - \\
\hline Si IV & 4116.10 & - & - & - & - & - & - & - & - & 77 & 7.32 \\
\hline Si IV & 4212.00 & - & - & - & - & - & - & - & - & - & - \\
\hline
\end{tabular}


I. Hunter et al.: Chemical compositions of B-type stars, Online Material $p 12$

Table 1. continued.

\begin{tabular}{|c|c|c|c|c|c|c|c|c|c|c|c|}
\hline \multirow[t]{2}{*}{ Species } & \multirow[t]{2}{*}{ Wavelength } & \multicolumn{2}{|c|}{ NGC 4755-006 } & \multicolumn{2}{|c|}{ NGC 4755-015 } & \multicolumn{2}{|c|}{ NGC 4755-016 } & \multicolumn{2}{|c|}{ NGC 4755-017 } & \multicolumn{2}{|c|}{ NGC 4755-020 } \\
\hline & & $E W$ & Abund & $E W$ & Abund & $E W$ & Abund & $E W$ & Abund & $E W$ & Abund \\
\hline C II & 4267.00 & 223 & 7.66 & 165 & 7.90 & 177 & 7.98 & 203 & 8.04 & 213 & 8.22 \\
\hline N II & 3955.85 & - & - & 40 & 7.96 & - & - & - & - & 15 & 7.57 \\
\hline N II & 3995.00 & 131 & 7.39 & 117 & 7.74 & - & - & 61 & 7.53 & 67 & 7.51 \\
\hline N II & 4447.03 & - & - & 45 & 7.46 & - & - & 52 & 8.02 & - & - \\
\hline N II & 4601.48 & 88 & 7.81 & - & - & - & - & - & - & 40 & 7.85 \\
\hline $\mathrm{N}$ II & 4613.86 & 54 & 7.75 & - & - & - & - & - & - & 24 & 7.71 \\
\hline N II & 4630.54 & 127 & 7.58 & 117 & 8.00 & 82 & 7.64 & 49 & 7.65 & 58 & 7.67 \\
\hline N II & 4643.08 & - & - & 64 & 7.91 & - & - & 30 & 7.75 & 37 & 7.76 \\
\hline O II & 3912.00 & 120 & 8.77 & 96 & 8.90 & - & - & - & - & 39 & 8.52 \\
\hline O II & 3919.30 & - & - & - & - & - & - & - & - & - & - \\
\hline O II & 3945.04 & 96 & 8.83 & 50 & 8.63 & - & - & - & - & 27 & 8.56 \\
\hline O II & 3954.36 & 129 & 8.73 & 80 & 8.74 & - & - & - & - & 37 & 8.53 \\
\hline O II & 3982.71 & 104 & 8.90 & - & - & - & - & - & - & 20 & 8.45 \\
\hline O II & 4069.00 & 246 & 8.88 & 153 & 8.73 & - & - & 65 & 8.55 & 75 & 8.45 \\
\hline O II & 4072.15 & 205 & 8.85 & 118 & 8.90 & - & - & 56 & 8.76 & 57 & 8.56 \\
\hline O II & 4075.86 & 251 & 8.91 & 116 & 8.68 & - & - & 78 & 8.97 & 86 & 8.89 \\
\hline O II & 4078.84 & - & - & 50 & 8.82 & - & - & - & - & 16 & 8.32 \\
\hline O II & 4132.80 & 118 & 8.99 & - & - & - & - & - & - & 22 & 8.35 \\
\hline O II & 4156.53 & 36 & 8.94 & 24 & 8.77 & - & - & - & - & 12 & 8.55 \\
\hline O II & 4185.44 & - & - & - & - & - & - & - & - & - & - \\
\hline O II & 4317.00 & 164 & 9.03 & 107 & 9.12 & 80 & 8.81 & 46 & 8.91 & 47 & 8.71 \\
\hline O II & 4319.63 & 172 & 9.08 & 87 & 8.86 & 75 & 8.74 & 31 & 8.56 & 38 & 8.51 \\
\hline O II & 4325.76 & - & - & - & - & - & - & - & - & - & - \\
\hline O II & 4349.43 & - & - & 104 & 8.77 & - & - & - & - & - & - \\
\hline O II & 4351.00 & - & - & 86 & 8.73 & - & - & - & - & - & - \\
\hline O II & 4353.58 & - & - & - & - & - & - & - & - & - & - \\
\hline O II & 4366.00 & 197 & 9.14 & 93 & 8.85 & - & - & 40 & 8.71 & 41 & 8.52 \\
\hline O II & 4369.27 & - & - & 33 & 8.75 & - & - & - & - & - & - \\
\hline O II & 4395.94 & - & - & 60 & 9.04 & - & - & 33 & 9.24 & 34 & 9.03 \\
\hline O II & 4414.90 & 204 & 8.73 & 123 & 8.71 & 68 & 8.12 & 40 & 8.27 & 62 & 8.47 \\
\hline O II & 4416.98 & 150 & 8.68 & 117 & 8.96 & 124 & 9.08 & 43 & 8.64 & 51 & 8.59 \\
\hline O II & 4443.01 & - & - & - & - & - & - & - & - & - & - \\
\hline O II & 4452.38 & 85 & 9.00 & 46 & 8.80 & - & - & - & - & 23 & 8.66 \\
\hline O II & 4590.97 & 171 & 8.88 & 91 & 8.74 & 91 & 8.79 & 46 & 8.87 & 36 & 8.38 \\
\hline O II & 4596.18 & 156 & 8.90 & 103 & 8.91 & 73 & 8.62 & 40 & 8.80 & 36 & 8.45 \\
\hline O II & 4638.86 & 178 & 8.99 & 100 & 8.91 & - & - & 40 & 8.75 & 46 & 8.64 \\
\hline O II & 4641.81 & - & - & 124 & 8.70 & - & - & 41 & 8.34 & 61 & 8.50 \\
\hline O II & 4650.00 & - & - & 190 & 8.45 & - & - & - & - & 57 & 7.83 \\
\hline O II & 4661.63 & 198 & 9.01 & 99 & 8.81 & 52 & 8.23 & 34 & 8.55 & 40 & 8.44 \\
\hline O II & 4673.73 & 44 & 8.80 & 39 & 8.93 & - & - & - & - & 15 & 8.65 \\
\hline O II & 4676.23 & 204 & 9.18 & 93 & 8.89 & - & - & - & - & 36 & 8.47 \\
\hline O II & 4699.00 & 113 & 8.65 & 96 & 8.70 & - & - & - & - & 37 & 8.35 \\
\hline O II & 4705.35 & 136 & 8.79 & 84 & 8.71 & - & - & - & - & 35 & 8.41 \\
\hline O II & 4710.00 & 42 & 8.89 & 57 & 9.38 & - & - & - & - & 18 & 8.95 \\
\hline Mg II & 4481.00 & 230 & 7.21 & 131 & 7.09 & - & - & 184 & 7.32 & 167 & 7.44 \\
\hline Si II & 4128.05 & - & - & - & - & - & - & 50 & 7.64 & 34 & 7.51 \\
\hline Si II & 4130.89 & - & - & - & - & - & - & 51 & 7.37 & 38 & 7.36 \\
\hline Si III & 4552.62 & 357 & 7.38 & 179 & 7.46 & 173 & 7.45 & 111 & 7.51 & 111 & 7.44 \\
\hline Si III & 4567.84 & 304 & 7.43 & 144 & 7.41 & 131 & 7.32 & 79 & 7.36 & 83 & 7.32 \\
\hline Si III & 4574.75 & 196 & 7.49 & 104 & 7.53 & 107 & 7.60 & 52 & 7.44 & 56 & 7.40 \\
\hline Si IV & 4088.90 & - & - & - & - & - & - & - & - & - & - \\
\hline Si IV & 4116.10 & 41 & 7.25 & 30 & 7.46 & - & - & - & - & - & - \\
\hline Si IV & 4212.00 & - & - & - & - & - & - & - & - & - & - \\
\hline
\end{tabular}


I. Hunter et al.: Chemical compositions of B-type stars, Online Material p 13

Table 1. continued.

\begin{tabular}{|c|c|c|c|c|c|c|c|c|c|c|c|}
\hline \multirow[t]{2}{*}{ Species } & \multirow[t]{2}{*}{ Wavelength } & \multicolumn{2}{|c|}{ NGC 4755-023 } & \multicolumn{2}{|c|}{ NGC 4755-026 } & \multicolumn{2}{|c|}{ NGC 4755-029 } & \multicolumn{2}{|c|}{ NGC 4755-033 } & \multicolumn{2}{|c|}{ NGC 4755-040 } \\
\hline & & $E W$ & Abund & $E W$ & Abund & $E W$ & Abund & $E W$ & Abund & $E W$ & Abund \\
\hline C II & 4267.00 & 213 & 8.17 & 212 & 8.08 & 213 & 8.21 & 190 & 8.07 & 219 & 8.29 \\
\hline N II & 3955.85 & - & - & - & - & - & - & - & - & - & - \\
\hline N II & 3995.00 & 40 & 7.35 & - & - & 46 & 7.47 & 21 & 7.23 & 39 & 7.58 \\
\hline N II & 4447.03 & 35 & 7.91 & - & - & - & - & - & - & - & - \\
\hline N II & 4601.48 & 25 & 7.82 & - & - & 25 & 7.80 & - & - & - & - \\
\hline N II & 4613.86 & - & - & - & - & - & - & - & - & - & - \\
\hline N II & 4630.54 & 54 & 7.99 & 24 & 7.47 & 42 & 7.74 & 21 & 7.63 & 33 & 7.81 \\
\hline N II & 4643.08 & - & - & - & - & - & - & - & - & - & - \\
\hline O II & 3912.00 & - & - & - & - & - & - & - & - & - & - \\
\hline O II & 3919.30 & - & - & - & - & - & - & - & - & - & - \\
\hline O II & 3945.04 & - & - & - & - & - & - & - & - & - & - \\
\hline O II & 3954.36 & - & - & - & - & - & - & - & - & - & - \\
\hline O II & 3982.71 & - & - & - & - & - & - & - & - & - & - \\
\hline O II & 4069.00 & - & - & - & - & - & - & 22 & 8.53 & 36 & 8.64 \\
\hline O II & 4072.15 & - & - & - & - & - & - & 18 & 8.57 & 36 & 8.94 \\
\hline O II & 4075.86 & - & - & - & - & - & - & 35 & 8.95 & 63 & 9.35 \\
\hline O II & 4078.84 & - & - & - & - & - & - & - & - & - & - \\
\hline O II & 4132.80 & - & - & - & - & - & - & - & - & - & - \\
\hline O II & 4156.53 & - & - & - & - & - & - & - & - & - & - \\
\hline O II & 4185.44 & - & - & - & - & - & - & - & - & - & - \\
\hline O II & 4317.00 & - & - & - & - & 24 & 8.56 & - & - & - & - \\
\hline O II & 4319.63 & - & - & - & - & 20 & 8.42 & - & - & - & - \\
\hline O II & 4325.76 & - & - & - & - & - & - & - & - & - & - \\
\hline O II & 4349.43 & - & - & - & - & - & - & - & - & - & - \\
\hline O II & 4351.00 & - & - & - & - & - & - & - & - & - & - \\
\hline O II & 4353.58 & - & - & - & - & - & - & - & - & - & - \\
\hline O II & 4366.00 & - & - & - & - & - & - & - & - & - & - \\
\hline O II & 4369.27 & - & - & - & - & - & - & - & - & - & - \\
\hline O II & 4395.94 & - & - & - & - & - & - & - & - & - & - \\
\hline O II & 4414.90 & - & - & - & - & 21 & 7.96 & - & - & - & - \\
\hline O II & 4416.98 & - & - & - & - & 24 & 8.37 & - & - & - & - \\
\hline O II & 4443.01 & - & - & - & - & - & - & - & - & - & - \\
\hline O II & 4452.38 & - & - & - & - & - & - & - & - & - & - \\
\hline O II & 4590.97 & 32 & 8.84 & - & - & 35 & 8.88 & - & - & - & - \\
\hline O II & 4596.18 & 31 & 8.87 & - & - & 18 & 8.41 & - & - & - & - \\
\hline O II & 4638.86 & - & - & - & - & - & - & - & - & - & - \\
\hline O II & 4641.81 & - & - & - & - & - & - & - & - & - & - \\
\hline O II & 4650.00 & 88 & 8.75 & - & - & 82 & 8.65 & - & - & - & - \\
\hline O II & 4661.63 & - & - & - & - & - & - & - & - & - & - \\
\hline O II & 4673.73 & - & - & - & - & - & - & - & - & - & - \\
\hline O II & 4676.23 & - & - & - & - & - & - & - & - & - & - \\
\hline O II & 4699.00 & - & - & - & - & - & - & - & - & - & - \\
\hline O II & 4705.35 & - & - & - & - & 25 & 8.63 & - & - & - & - \\
\hline O II & 4710.00 & - & - & - & - & - & - & - & - & - & - \\
\hline Mg II & 4481.00 & 188 & 7.41 & 175 & 6.93 & 180 & 7.45 & 233 & 7.09 & 185 & 7.19 \\
\hline Si II & 4128.05 & - & - & - & - & - & - & 75 & 7.42 & 51 & 7.62 \\
\hline Si II & 4130.89 & - & - & - & - & - & - & 77 & 7.18 & 54 & 7.36 \\
\hline Si III & 4552.62 & 84 & 7.51 & 85 & 7.46 & 81 & 7.49 & 62 & 7.44 & 62 & 7.40 \\
\hline Si III & 4567.84 & 68 & 7.52 & - & - & 53 & 7.26 & 35 & 7.19 & 49 & 7.41 \\
\hline Si III & 4574.75 & 33 & 7.30 & - & - & 40 & 7.49 & 24 & 7.39 & 32 & 7.52 \\
\hline Si IV & 4088.90 & - & - & - & - & - & - & - & - & - & - \\
\hline Si IV & 4116.10 & - & - & - & - & - & - & - & - & - & - \\
\hline Si IV & 4212.00 & - & - & - & - & - & - & - & - & - & - \\
\hline
\end{tabular}


I. Hunter et al.: Chemical compositions of B-type stars, Online Material p 14

Table 1. continued.

\begin{tabular}{|c|c|c|c|c|c|c|c|c|c|c|c|}
\hline \multirow[t]{2}{*}{ Species } & \multirow[t]{2}{*}{ Wavelength } & \multicolumn{2}{|c|}{ NGC 4755-048 } & \multicolumn{2}{|c|}{ NGC 346-012 } & \multicolumn{2}{|c|}{ NGC 346-021 } & \multicolumn{2}{|c|}{ NGC 346-026 } & \multicolumn{2}{|c|}{ NGC 346-027 } \\
\hline & & $E W$ & Abund & $E W$ & Abund & $E W$ & Abund & $E W$ & Abund & $E W$ & Abund \\
\hline C II & 4267.00 & 207 & 8.22 & 62 & 7.45 & 63 & 7.52 & - & - & - & - \\
\hline N II & 3955.85 & - & - & - & - & - & - & - & - & - & - \\
\hline N II & 3995.00 & 24 & 7.30 & 46 & 6.94 & 33 & 6.81 & - & - & $<63$ & $<7.71$ \\
\hline N II & 4447.03 & - & - & - & - & - & - & - & - & - & - \\
\hline N II & 4601.48 & - & - & - & - & - & - & - & - & - & - \\
\hline N II & 4613.86 & - & - & - & - & - & - & - & - & - & - \\
\hline N II & 4630.54 & 20 & 7.59 & 27 & 6.92 & 23 & 6.87 & 38 & 7.79 & - & - \\
\hline N II & 4643.08 & - & - & - & - & - & - & - & - & - & - \\
\hline O II & 3912.00 & - & - & 57 & 8.04 & 61 & 8.19 & - & - & - & - \\
\hline O II & 3919.30 & - & - & - & - & - & - & - & - & - & - \\
\hline O II & 3945.04 & - & - & 34 & 8.02 & 34 & 8.11 & - & - & - & - \\
\hline O II & 3954.36 & - & - & 55 & 7.97 & 51 & 8.03 & - & - & - & - \\
\hline O II & 3982.71 & - & - & 33 & 8.02 & 43 & 8.27 & - & - & - & - \\
\hline O II & 4069.00 & 29 & 8.69 & 154 & 8.15 & 130 & 8.19 & - & - & - & - \\
\hline O II & 4072.15 & 30 & 8.96 & 107 & 8.03 & 83 & 8.08 & 55 & 8.03 & - & - \\
\hline O II & 4075.86 & 50 & 9.28 & 126 & 8.00 & 88 & 7.96 & 56 & 7.87 & - & - \\
\hline O II & 4078.84 & - & - & 32 & 8.05 & 35 & 8.24 & - & - & - & - \\
\hline O II & 4132.80 & 15 & 9.08 & 45 & 8.11 & 39 & 8.10 & - & - & - & - \\
\hline O II & 4156.53 & - & - & - & - & - & - & - & - & - & - \\
\hline O II & 4185.44 & - & - & 37 & 7.91 & 42 & 8.06 & - & - & - & - \\
\hline O II & 4317.00 & - & - & 82 & 8.26 & 71 & 8.35 & - & - & - & - \\
\hline O II & 4319.63 & - & - & 96 & 8.38 & 73 & 8.38 & - & - & - & - \\
\hline O II & 4325.76 & - & - & 26 & 8.31 & 24 & 8.35 & - & - & - & - \\
\hline O II & 4349.43 & - & - & 149 & 8.38 & 98 & 8.30 & - & - & - & - \\
\hline O II & 4351.00 & - & - & 81 & 7.98 & 66 & 7.98 & - & - & - & - \\
\hline O II & 4353.58 & - & - & - & - & - & - & - & - & - & - \\
\hline O II & 4366.00 & - & - & 80 & 8.15 & 67 & 8.21 & - & - & - & - \\
\hline O II & 4369.27 & - & - & 17 & 8.08 & 12 & 7.91 & - & - & - & - \\
\hline O II & 4395.94 & - & - & - & - & - & - & - & - & - & - \\
\hline O II & 4414.90 & - & - & 129 & 8.17 & 104 & 8.21 & - & - & 67 & 8.14 \\
\hline O II & 4416.98 & - & - & 95 & 8.17 & 89 & 8.34 & - & - & 54 & 8.24 \\
\hline O II & 4443.01 & - & - & - & - & - & - & - & - & - & - \\
\hline O II & 4452.38 & - & - & 29 & 8.20 & 35 & 8.35 & - & - & - & - \\
\hline O II & 4590.97 & - & - & 89 & 8.06 & 71 & 8.09 & - & - & 59 & 8.11 \\
\hline O II & 4596.18 & - & - & 79 & 8.08 & 70 & 8.16 & - & - & 51 & 8.13 \\
\hline O II & 4638.86 & - & - & 106 & 8.31 & 71 & 8.18 & - & - & - & - \\
\hline O II & 4641.81 & - & - & 139 & 8.10 & 105 & 8.12 & - & - & - & - \\
\hline O II & 4650.00 & - & - & - & - & 196 & 8.12 & - & - & - & - \\
\hline O II & 4661.63 & - & - & 113 & 8.28 & 78 & 8.19 & - & - & 60 & 8.26 \\
\hline O II & 4673.73 & - & - & - & - & - & - & - & - & - & - \\
\hline O II & 4676.23 & - & - & 79 & 8.14 & 60 & 8.09 & - & - & - & - \\
\hline O II & 4699.00 & - & - & - & - & - & - & - & - & - & - \\
\hline O II & 4705.35 & - & - & 73 & 8.07 & 59 & 8.04 & 27 & 7.91 & - & - \\
\hline O II & 4710.00 & - & - & - & - & - & - & - & - & - & - \\
\hline Mg II & 4481.00 & 202 & 7.08 & 55 & 6.70 & 55 & 6.76 & 29 & 6.65 & 59 & 7.00 \\
\hline Si II & 4128.05 & 60 & 7.44 & - & - & - & - & - & - & - & - \\
\hline Si II & 4130.89 & 72 & 7.41 & - & - & - & - & - & - & - & - \\
\hline Si III & 4552.62 & 59 & 7.45 & 193 & 6.79 & 114 & 6.73 & 72 & 6.80 & 82 & 6.60 \\
\hline Si III & 4567.84 & 35 & 7.22 & 157 & 6.83 & 93 & 6.74 & 51 & 6.78 & 70 & 6.76 \\
\hline Si III & 4574.75 & 27 & 7.50 & 84 & 6.84 & 59 & 6.86 & 22 & 6.79 & 47 & 7.07 \\
\hline Si IV & 4088.90 & - & - & - & - & - & - & - & - & - & - \\
\hline Si IV & 4116.10 & - & - & 70 & 6.86 & 42 & 6.87 & 112 & 6.80 & - & - \\
\hline Si IV & 4212.00 & - & - & - & - & - & - & - & - & - & - \\
\hline
\end{tabular}


I. Hunter et al.: Chemical compositions of B-type stars, Online Material p 15

Table 1. continued.

\begin{tabular}{|c|c|c|c|c|c|c|c|c|c|c|c|}
\hline \multirow[t]{2}{*}{ Species } & \multirow[t]{2}{*}{ Wavelength } & \multicolumn{2}{|c|}{ NGC 346-029 } & \multicolumn{2}{|c|}{ NGC 346-032 } & \multicolumn{2}{|c|}{ NGC 346-037 } & \multicolumn{2}{|c|}{ NGC 346-039 } & \multicolumn{2}{|c|}{ NGC 346-040 } \\
\hline & & $E W$ & Abund & $E W$ & Abund & $E W$ & Abund & $E W$ & Abund & $E W$ & Abund \\
\hline C II & 4267.00 & 16 & 7.33 & 42 & 7.44 & 79 & 7.00 & 63 & 7.54 & 24 & 7.28 \\
\hline N II & 3955.85 & - & - & - & - & - & - & - & - & - & - \\
\hline N II & 3995.00 & $<13$ & $<6.99$ & $<25$ & $<6.88$ & 64 & 7.58 & $<21$ & $<6.59$ & $<16$ & $<6.88$ \\
\hline N II & 4447.03 & - & - & - & - & 25 & 7.49 & - & - & - & - \\
\hline N II & 4601.48 & - & - & - & - & 26 & 7.67 & - & - & - & - \\
\hline N II & 4613.86 & - & - & - & - & - & - & - & - & - & - \\
\hline $\mathrm{N}$ II & 4630.54 & - & - & - & - & 39 & 7.48 & - & - & - & - \\
\hline N II & 4643.08 & - & - & - & - & - & - & - & - & - & - \\
\hline O II & 3912.00 & - & - & 26 & 7.81 & - & - & 61 & 8.21 & - & - \\
\hline O II & 3919.30 & - & - & - & - & - & - & - & - & - & - \\
\hline O II & 3945.04 & - & - & - & - & - & - & 44 & 8.32 & - & - \\
\hline O II & 3954.36 & - & - & - & - & 11 & 7.90 & 55 & 8.14 & - & - \\
\hline O II & 3982.71 & - & - & - & - & - & - & 37 & 8.21 & - & - \\
\hline O II & 4069.00 & 87 & 8.23 & 75 & 7.85 & 28 & 7.95 & 141 & 8.31 & 85 & 8.05 \\
\hline O II & 4072.15 & 39 & 7.83 & 67 & 8.06 & 24 & 8.07 & 87 & 8.18 & 44 & 7.75 \\
\hline O II & 4075.86 & 49 & 7.84 & 50 & 7.59 & - & - & 100 & 8.15 & 50 & 7.69 \\
\hline O II & 4078.84 & - & - & - & - & - & - & 40 & 8.37 & - & - \\
\hline O II & 4132.80 & - & - & - & - & - & - & 43 & 8.22 & - & - \\
\hline O II & 4156.53 & - & - & - & - & - & - & - & - & - & - \\
\hline O II & 4185.44 & - & - & - & - & - & - & 48 & 8.16 & - & - \\
\hline O II & 4317.00 & 30 & 8.29 & 26 & 7.77 & - & - & 65 & 8.30 & 34 & 8.16 \\
\hline O II & 4319.63 & 32 & 8.32 & 36 & 7.98 & - & - & 72 & 8.41 & 35 & 8.17 \\
\hline O II & 4325.76 & - & - & - & - & - & - & 32 & 8.59 & 13 & 8.35 \\
\hline O II & 4349.43 & 35 & 8.16 & - & - & 23 & 8.10 & 106 & 8.48 & - & - \\
\hline O II & 4351.00 & 25 & 7.60 & - & - & - & - & 65 & 7.99 & - & - \\
\hline O II & 4353.58 & - & - & - & - & - & - & - & - & - & - \\
\hline O II & 4366.00 & 27 & 8.20 & - & - & - & - & 67 & 8.25 & 35 & 8.11 \\
\hline O II & 4369.27 & - & - & - & - & - & - & 15 & 8.07 & - & - \\
\hline O II & 4395.94 & - & - & - & - & - & - & - & - & - & - \\
\hline O II & 4414.90 & 29 & 7.95 & 62 & 7.96 & 19 & 7.76 & 105 & 8.26 & 43 & 7.90 \\
\hline O II & 4416.98 & 25 & 8.10 & 42 & 7.93 & - & - & 75 & 8.23 & 37 & 8.06 \\
\hline O II & 4443.01 & - & - & - & - & - & - & - & - & - & - \\
\hline O II & 4452.38 & - & - & - & - & - & - & 37 & 8.41 & - & - \\
\hline O II & 4590.97 & 22 & 7.81 & 37 & 7.79 & - & - & 76 & 8.18 & 28 & 7.69 \\
\hline O II & 4596.18 & 22 & 7.90 & 29 & 7.74 & - & - & 70 & 8.19 & 26 & 7.78 \\
\hline O II & 4638.86 & 22 & 8.04 & 49 & 8.13 & 16 & 8.06 & 73 & 8.25 & 30 & 7.96 \\
\hline O II & 4641.81 & 45 & 8.09 & 57 & 7.84 & 23 & 7.91 & 108 & 8.21 & 52 & 7.95 \\
\hline O II & 4650.00 & 85 & 8.09 & - & - & 37 & 7.77 & 208 & 8.23 & 89 & 7.87 \\
\hline O II & 4661.63 & 28 & 8.11 & 35 & 7.81 & - & - & 78 & 8.24 & 38 & 8.04 \\
\hline O II & 4673.73 & - & - & - & - & - & - & - & - & - & - \\
\hline O II & 4676.23 & 23 & 8.11 & - & - & - & - & 66 & 8.21 & 29 & 7.97 \\
\hline O II & 4699.00 & - & - & - & - & - & - & - & - & - & - \\
\hline O II & 4705.35 & 18 & 7.70 & - & - & - & - & 60 & 8.08 & 26 & 7.73 \\
\hline O II & 4710.00 & - & - & - & - & - & - & - & - & - & - \\
\hline Mg II & 4481.00 & 28 & 6.69 & - & - & 96 & 6.62 & 50 & 6.73 & 18 & 6.39 \\
\hline Si II & 4128.05 & - & - & - & - & 27 & 6.88 & - & - & - & - \\
\hline Si II & 4130.89 & - & - & - & - & 24 & 6.56 & - & - & - & - \\
\hline Si III & 4552.62 & 44 & 6.62 & 63 & 6.57 & 61 & 6.86 & 105 & 6.68 & 47 & 6.43 \\
\hline Si III & 4567.84 & 38 & 6.76 & 46 & 6.52 & 41 & 6.71 & 91 & 6.79 & 40 & 6.56 \\
\hline Si III & 4574.75 & 15 & 6.69 & 27 & 6.65 & 24 & 6.79 & 58 & 6.90 & 21 & 6.69 \\
\hline Si IV & 4088.90 & - & - & - & - & - & - & - & - & - & - \\
\hline Si IV & 4116.10 & 69 & 6.70 & - & - & - & - & 46 & 6.85 & 61 & 6.70 \\
\hline Si IV & 4212.00 & - & - & - & - & - & - & - & - & 10 & 6.52 \\
\hline
\end{tabular}


I. Hunter et al.: Chemical compositions of B-type stars, Online Material p 16

Table 1. continued.

\begin{tabular}{|c|c|c|c|c|c|c|c|c|c|c|c|}
\hline \multirow[t]{2}{*}{ Species } & \multirow[t]{2}{*}{ Wavelength } & \multicolumn{2}{|c|}{ NGC 346-043 } & \multicolumn{2}{|c|}{ NGC 346-044 } & \multicolumn{2}{|c|}{ NGC 346-047 } & \multicolumn{2}{|c|}{ NGC 346-053 } & \multicolumn{2}{|c|}{ NGC 346-054 } \\
\hline & & $E W$ & Abund & $E W$ & Abund & $E W$ & Abund & $E W$ & Abund & $E W$ & Abund \\
\hline C II & 4267.00 & 15 & 7.37 & 84 & 7.50 & 92 & 7.18 & 58 & 7.77 & 32 & 7.29 \\
\hline N II & 3955.85 & - & - & - & - & - & - & - & - & - & - \\
\hline N II & 3995.00 & $<7$ & $<6.75$ & $<42$ & $<6.83$ & 55 & 7.29 & $<45$ & $<7.44$ & $<20$ & $<6.77$ \\
\hline N II & 4447.03 & - & - & $<24$ & $<7.05$ & 26 & 7.36 & - & - & - & - \\
\hline N II & 4601.48 & - & - & - & - & 25 & 7.49 & - & - & - & - \\
\hline N II & 4613.86 & - & - & - & - & 18 & 7.52 & - & - & - & - \\
\hline N II & 4630.54 & - & - & - & - & 47 & 7.46 & - & - & - & - \\
\hline N II & 4643.08 & - & - & - & - & 20 & 7.32 & - & - & - & - \\
\hline O II & 3912.00 & 16 & 7.87 & 51 & 8.25 & - & - & - & - & 27 & 7.81 \\
\hline O II & 3919.30 & - & - & - & - & - & - & - & - & - & - \\
\hline O II & 3945.04 & - & - & 28 & 8.16 & - & - & - & - & 16 & 7.87 \\
\hline O II & 3954.36 & - & - & 46 & 8.16 & - & - & - & - & 21 & 7.70 \\
\hline O II & 3982.71 & - & - & - & - & - & - & - & - & 17 & 7.93 \\
\hline O II & 4069.00 & - & - & 92 & 8.09 & 32 & 7.83 & 138 & 8.54 & 59 & 7.65 \\
\hline O II & 4072.15 & 42 & 7.94 & 81 & 8.32 & - & - & 98 & 8.67 & 32 & 7.40 \\
\hline O II & 4075.86 & 55 & 7.98 & 91 & 8.27 & 27 & 7.75 & 63 & 7.85 & 43 & 7.44 \\
\hline O II & 4078.84 & 17 & 8.22 & 29 & 8.29 & - & - & - & - & 11 & 7.62 \\
\hline O II & 4132.80 & 15 & 7.94 & - & - & - & - & - & - & - & - \\
\hline O II & 4156.53 & - & - & - & - & - & - & - & - & - & - \\
\hline O II & 4185.44 & - & - & - & - & - & - & - & - & - & - \\
\hline O II & 4317.00 & 17 & 8.07 & 47 & 8.16 & 16 & 7.86 & - & - & 25 & 7.74 \\
\hline O II & 4319.63 & 22 & 8.19 & 52 & 8.24 & - & - & - & - & 29 & 7.83 \\
\hline O II & 4325.76 & - & - & - & - & - & - & - & - & - & - \\
\hline O II & 4349.43 & 50 & 8.44 & 72 & 8.21 & - & - & - & - & 48 & 7.94 \\
\hline O II & 4351.00 & 16 & 7.44 & 39 & 7.92 & - & - & - & - & 36 & 7.70 \\
\hline O II & 4353.58 & - & - & - & - & - & - & - & - & - & - \\
\hline O II & 4366.00 & 23 & 8.18 & 45 & 8.03 & - & - & 56 & 8.45 & 25 & 7.69 \\
\hline O II & 4369.27 & - & - & - & - & - & - & - & - & - & - \\
\hline O II & 4395.94 & - & - & - & - & - & - & - & - & - & - \\
\hline O II & 4414.90 & 29 & 8.01 & 74 & 8.09 & 26 & 7.79 & - & - & 42 & 7.66 \\
\hline O II & 4416.98 & 15 & 7.92 & 59 & 8.17 & - & - & - & - & 36 & 7.83 \\
\hline O II & 4443.01 & - & - & - & - & - & - & - & - & - & - \\
\hline O II & 4452.38 & - & - & - & - & - & - & - & - & - & - \\
\hline O II & 4590.97 & 8 & 7.41 & 45 & 7.99 & - & - & - & - & 37 & 7.77 \\
\hline O II & 4596.18 & 15 & 7.81 & 40 & 8.00 & - & - & - & - & 31 & 7.77 \\
\hline O II & 4638.86 & 25 & 8.18 & 58 & 8.24 & 15 & 7.82 & 73 & 8.57 & 34 & 7.85 \\
\hline O II & 4641.81 & - & - & 72 & 7.98 & 25 & 7.76 & 82 & 8.27 & 48 & 7.69 \\
\hline O II & 4650.00 & 89 & 8.22 & - & - & 50 & 7.78 & - & - & - & - \\
\hline O II & 4661.63 & 28 & 8.19 & 48 & 8.01 & - & - & 62 & 8.34 & 33 & 7.76 \\
\hline O II & 4673.73 & - & - & - & - & - & - & - & - & 14 & 8.19 \\
\hline O II & 4676.23 & 22 & 8.17 & 47 & 8.12 & - & - & 63 & 8.47 & 33 & 7.87 \\
\hline O II & 4699.00 & - & - & - & - & - & - & - & - & 23 & 7.55 \\
\hline O II & 4705.35 & 18 & 7.74 & 37 & 7.96 & - & - & - & - & 29 & 7.65 \\
\hline O II & 4710.00 & - & - & - & - & - & - & - & - & - & - \\
\hline Mg II & 4481.00 & 35 & 6.83 & 62 & 6.66 & 94 & 6.74 & 43 & 6.84 & 25 & 6.45 \\
\hline Si II & 4128.05 & - & - & - & - & 19 & 6.75 & - & - & - & - \\
\hline Si II & 4130.89 & - & - & - & - & 23 & 6.66 & - & - & - & - \\
\hline Si III & 4552.62 & 43 & 6.72 & 101 & 6.62 & 75 & 6.93 & 74 & 6.80 & 48 & 6.27 \\
\hline Si III & 4567.84 & 32 & 6.73 & 86 & 6.70 & 50 & 6.74 & - & - & 42 & 6.42 \\
\hline Si III & 4574.75 & 11 & 6.63 & 65 & 6.97 & 25 & 6.65 & - & - & 23 & 6.53 \\
\hline Si IV & 4088.90 & - & - & - & - & - & - & - & - & - & - \\
\hline Si IV & 4116.10 & 83 & 7.02 & - & - & - & - & - & - & 26 & 6.44 \\
\hline Si IV & 4212.00 & 15 & 6.55 & - & - & - & - & - & - & - & - \\
\hline
\end{tabular}


I. Hunter et al.: Chemical compositions of B-type stars, Online Material p 17

Table 1. continued.

\begin{tabular}{|c|c|c|c|c|c|c|c|c|c|c|c|}
\hline \multirow[t]{2}{*}{ Species } & \multirow[t]{2}{*}{ Wavelength } & \multicolumn{2}{|c|}{ NGC 346-055 } & \multicolumn{2}{|c|}{ NGC 346-056 } & \multicolumn{2}{|c|}{ NGC 346-057 } & \multicolumn{2}{|c|}{ NGC 346-058 } & \multicolumn{2}{|c|}{ NGC 346-062 } \\
\hline & & $E W$ & Abund & $E W$ & Abund & $E W$ & Abund & $E W$ & Abund & $E W$ & Abund \\
\hline C II & 4267.00 & 40 & 7.45 & 14 & 7.15 & 91 & 7.16 & - & - & 32 & 7.35 \\
\hline N II & 3955.85 & - & - & - & - & - & - & - & - & - & - \\
\hline N II & 3995.00 & $<40$ & $<7.25$ & 33 & 7.47 & $<25$ & $<6.71$ & $<70$ & $<7.56$ & 36 & 7.20 \\
\hline N II & 4447.03 & - & - & 15 & 7.31 & - & - & - & - & - & - \\
\hline N II & 4601.48 & - & - & - & - & - & - & - & - & - & - \\
\hline N II & 4613.86 & - & - & - & - & - & - & - & - & - & - \\
\hline N II & 4630.54 & - & - & 21 & 7.43 & - & - & - & - & 29 & 7.34 \\
\hline N II & 4643.08 & - & - & - & - & - & - & - & - & 10 & 7.29 \\
\hline O II & 3912.00 & - & - & 20 & 7.86 & - & - & - & - & 31 & 7.86 \\
\hline O II & 3919.30 & - & - & - & - & - & - & - & - & - & - \\
\hline O II & 3945.04 & - & - & - & - & - & - & - & - & 17 & 7.90 \\
\hline O II & 3954.36 & 38 & 8.02 & - & - & - & - & - & - & 28 & 7.84 \\
\hline O II & 3982.71 & - & - & - & - & - & - & - & - & 9 & 7.61 \\
\hline O II & 4069.00 & - & - & - & - & - & - & - & - & 93 & 7.90 \\
\hline O II & 4072.15 & - & - & 52 & 8.02 & - & - & - & - & 73 & 7.91 \\
\hline O II & 4075.86 & - & - & 54 & 7.87 & - & - & - & - & 82 & 7.84 \\
\hline O II & 4078.84 & - & - & - & - & - & - & - & - & - & - \\
\hline O II & 4132.80 & - & - & - & - & - & - & - & - & - & - \\
\hline O II & 4156.53 & - & - & - & - & - & - & - & - & - & - \\
\hline O II & 4185.44 & - & - & - & - & - & - & - & - & - & - \\
\hline O II & 4317.00 & - & - & 20 & 8.09 & - & - & 38 & 7.97 & 28 & 7.90 \\
\hline O II & 4319.63 & - & - & 24 & 8.19 & - & - & 37 & 7.95 & 33 & 7.98 \\
\hline O II & 4325.76 & - & - & - & - & - & - & - & - & - & - \\
\hline O II & 4349.43 & - & - & 50 & 8.34 & - & - & - & - & 68 & 8.12 \\
\hline O II & 4351.00 & - & - & 25 & 7.56 & - & - & - & - & 28 & 7.45 \\
\hline O II & 4353.58 & - & - & - & - & - & - & - & - & - & - \\
\hline O II & 4366.00 & - & - & 23 & 8.10 & - & - & - & - & 38 & 7.98 \\
\hline O II & 4369.27 & - & - & - & - & - & - & - & - & - & - \\
\hline O II & 4395.94 & - & - & - & - & - & - & - & - & - & - \\
\hline O II & 4414.90 & 69 & 8.02 & 32 & 7.96 & - & - & - & - & - & - \\
\hline O II & 4416.98 & 47 & 8.01 & 21 & 7.97 & - & - & - & - & - & - \\
\hline O II & 4443.01 & - & - & - & - & - & - & - & - & - & - \\
\hline O II & 4452.38 & - & - & - & - & - & - & - & - & - & - \\
\hline O II & 4590.97 & 67 & 8.15 & 26 & 7.84 & - & - & - & - & 42 & 7.78 \\
\hline O II & 4596.18 & 53 & 8.07 & 21 & 7.85 & - & - & - & - & 38 & 7.84 \\
\hline O II & 4638.86 & 58 & 8.20 & 25 & 8.09 & - & - & - & - & 50 & 8.07 \\
\hline O II & 4641.81 & 97 & 8.23 & - & - & - & - & - & - & 93 & 8.11 \\
\hline O II & 4650.00 & - & - & 90 & 8.11 & 79 & 8.17 & - & - & - & - \\
\hline O II & 4661.63 & - & - & 38 & 8.26 & 28 & 8.22 & 84 & 8.32 & 60 & 8.12 \\
\hline O II & 4673.73 & - & - & - & - & - & - & - & - & - & - \\
\hline O II & 4676.23 & - & - & 23 & 8.07 & - & - & - & - & 42 & 8.02 \\
\hline O II & 4699.00 & - & - & - & - & - & - & - & - & 43 & 7.91 \\
\hline O II & 4705.35 & - & - & 24 & 7.82 & - & - & - & - & 49 & 7.96 \\
\hline O II & 4710.00 & - & - & - & - & - & - & - & - & - & - \\
\hline Mg II & 4481.00 & 30 & 6.57 & 37 & 6.81 & 77 & 6.53 & - & - & 41 & 6.75 \\
\hline Si II & 4128.05 & - & - & - & - & - & - & - & - & - & - \\
\hline Si II & 4130.89 & - & - & - & - & - & - & - & - & - & - \\
\hline Si III & 4552.62 & 76 & 6.58 & 58 & 6.73 & 65 & 6.68 & 85 & 6.53 & 117 & 6.89 \\
\hline Si III & 4567.84 & 73 & 6.83 & 47 & 6.82 & 63 & 6.91 & 83 & 6.79 & 87 & 6.87 \\
\hline Si III & 4574.75 & 45 & 7.00 & 20 & 6.77 & 32 & 6.83 & 58 & 7.07 & 33 & 6.71 \\
\hline Si IV & 4088.90 & - & - & - & - & - & - & - & - & - & - \\
\hline Si IV & 4116.10 & - & - & 96 & 7.02 & - & - & - & - & 99 & 6.99 \\
\hline Si IV & 4212.00 & - & - & 12 & 6.48 & - & - & - & - & 14 & 6.79 \\
\hline
\end{tabular}


I. Hunter et al.: Chemical compositions of B-type stars, Online Material p 18

Table 1. continued.

\begin{tabular}{|c|c|c|c|c|c|c|c|c|c|c|c|}
\hline \multirow[t]{2}{*}{ Species } & \multirow[t]{2}{*}{ Wavelength } & \multicolumn{2}{|c|}{ NGC 346-070 } & \multicolumn{2}{|c|}{ NGC 346-074 } & \multicolumn{2}{|c|}{ NGC 346-075 } & \multicolumn{2}{|c|}{ NGC 346-079 } & \multicolumn{2}{|c|}{ NGC 346-080 } \\
\hline & & $E W$ & Abund & $E W$ & Abund & $E W$ & Abund & $E W$ & Abund & $E W$ & Abund \\
\hline C II & 4267.00 & - & - & 59 & 6.92 & 59 & 7.56 & - & - & - & - \\
\hline N II & 3955.85 & - & - & - & - & - & - & - & - & - & - \\
\hline N II & 3995.00 & $<50$ & $<7.50$ & 29 & 7.43 & $<15$ & $<6.48$ & $<90$ & $<7.88$ & $<100$ & $<7.85$ \\
\hline N II & 4447.03 & - & - & - & - & - & - & - & - & - & - \\
\hline N II & 4601.48 & - & - & - & - & - & - & - & - & - & - \\
\hline N II & 4613.86 & - & - & - & - & - & - & - & - & - & - \\
\hline N II & 4630.54 & - & - & 19 & 7.57 & - & - & - & - & - & - \\
\hline N II & 4643.08 & - & - & - & - & - & - & - & - & - & - \\
\hline O II & 3912.00 & - & - & - & - & 42 & 8.04 & - & - & - & - \\
\hline O II & 3919.30 & - & - & - & - & - & - & - & - & - & - \\
\hline O II & 3945.04 & - & - & - & - & 17 & 7.87 & - & - & - & - \\
\hline O II & 3954.36 & - & - & - & - & 37 & 7.99 & - & - & - & - \\
\hline O II & 3982.71 & - & - & - & - & 31 & 8.25 & - & - & - & - \\
\hline O II & 4069.00 & - & - & - & - & 96 & 7.99 & - & - & - & - \\
\hline O II & 4072.15 & - & - & - & - & 69 & 8.00 & - & - & - & - \\
\hline O II & 4075.86 & - & - & - & - & 79 & 7.97 & - & - & - & - \\
\hline O II & 4078.84 & - & - & - & - & - & - & - & - & - & - \\
\hline O II & 4132.80 & - & - & - & - & 42 & 8.26 & - & - & - & - \\
\hline O II & 4156.53 & - & - & - & - & - & - & - & - & - & - \\
\hline O II & 4185.44 & - & - & - & - & 42 & 8.11 & - & - & - & - \\
\hline O II & 4317.00 & 26 & 7.90 & - & - & 48 & 8.12 & - & - & - & - \\
\hline O II & 4319.63 & 40 & 8.15 & - & - & 49 & 8.14 & - & - & - & - \\
\hline O II & 4325.76 & - & - & - & - & 22 & 8.44 & - & - & - & - \\
\hline O II & 4349.43 & - & - & - & - & 69 & 8.19 & - & - & - & - \\
\hline O II & 4351.00 & - & - & - & - & 44 & 7.87 & - & - & - & - \\
\hline O II & 4353.58 & - & - & - & - & - & - & - & - & - & - \\
\hline O II & 4366.00 & - & - & - & - & 39 & 7.89 & - & - & - & - \\
\hline O II & 4369.27 & - & - & - & - & 14 & 8.07 & - & - & - & - \\
\hline O II & 4395.94 & - & - & - & - & - & - & - & - & - & - \\
\hline O II & 4414.90 & - & - & - & - & 76 & 8.04 & - & - & 85 & 8.18 \\
\hline O II & 4416.98 & - & - & - & - & 58 & 8.09 & - & - & 62 & 8.18 \\
\hline O II & 4443.01 & - & - & - & - & - & - & - & - & - & - \\
\hline O II & 4452.38 & - & - & - & - & 21 & 8.15 & - & - & - & - \\
\hline O II & 4590.97 & 41 & 7.86 & - & - & 49 & 7.93 & - & - & - & - \\
\hline O II & 4596.18 & 36 & 7.89 & - & - & 47 & 8.00 & - & - & - & - \\
\hline O II & 4638.86 & 52 & 8.20 & - & - & 53 & 8.11 & - & - & 68 & 8.38 \\
\hline O II & 4641.81 & 89 & 8.24 & - & - & 75 & 7.99 & - & - & 82 & 8.14 \\
\hline O II & 4650.00 & - & - & - & - & 140 & 7.98 & - & - & 191 & 8.39 \\
\hline O II & 4661.63 & 45 & 8.03 & - & - & 53 & 8.05 & - & - & - & - \\
\hline O II & 4673.73 & - & - & - & - & - & - & - & - & - & - \\
\hline O II & 4676.23 & - & - & - & - & 47 & 8.07 & - & - & - & - \\
\hline O II & 4699.00 & 44 & 7.95 & - & - & - & - & - & - & - & - \\
\hline O II & 4705.35 & 32 & 7.77 & - & - & 43 & 7.88 & - & - & - & - \\
\hline O II & 4710.00 & - & - & - & - & - & - & - & - & - & - \\
\hline $\mathrm{Mg}$ II & 4481.00 & 39 & 6.77 & 134 & 6.53 & 57 & 6.88 & - & - & - & - \\
\hline Si II & 4128.05 & - & - & 49 & 6.94 & - & - & - & - & - & - \\
\hline Si II & 4130.89 & - & - & 51 & 6.73 & - & - & - & - & - & - \\
\hline Si III & 4552.62 & 77 & 6.71 & 29 & 6.82 & 86 & 6.68 & 88 & 6.83 & 87 & 6.76 \\
\hline Si III & 4567.84 & 68 & 6.88 & - & - & 73 & 6.77 & - & - & - & - \\
\hline Si III & 4574.75 & - & - & - & - & 51 & 6.98 & - & - & - & - \\
\hline Si IV & 4088.90 & - & - & - & - & - & - & - & - & - & - \\
\hline Si IV & 4116.10 & - & - & - & - & 34 & 6.95 & - & - & - & - \\
\hline Si IV & 4212.00 & - & - & - & - & - & - & - & - & - & - \\
\hline
\end{tabular}


I. Hunter et al.: Chemical compositions of B-type stars, Online Material p 19

Table 1. continued.

\begin{tabular}{|c|c|c|c|c|c|c|c|c|c|c|c|}
\hline \multirow[t]{2}{*}{ Species } & \multirow[t]{2}{*}{ Wavelength } & \multicolumn{2}{|c|}{ NGC 346-081 } & \multicolumn{2}{|c|}{ NGC 346-082 } & \multicolumn{2}{|c|}{ NGC 346-083 } & \multicolumn{2}{|c|}{ NGC 346-084 } & \multicolumn{2}{|c|}{ NGC 346-088 } \\
\hline & & $E W$ & Abund & $E W$ & Abund & $E W$ & Abund & $E W$ & Abund & $E W$ & Abund \\
\hline C II & 4267.00 & 55 & 7.02 & 87 & 7.30 & - & - & 54 & 7.48 & 80 & 7.78 \\
\hline N II & 3955.85 & - & - & - & - & - & - & - & - & - & - \\
\hline N II & 3995.00 & $<80$ & $<7.52$ & $<65$ & $<7.55$ & $<90$ & $<7.73$ & $<40$ & $<7.06$ & $<35$ & $<6.94$ \\
\hline N II & 4447.03 & - & - & - & - & - & - & - & - & - & - \\
\hline N II & 4601.48 & - & - & - & - & - & - & - & - & - & - \\
\hline $\mathrm{N}$ II & 4613.86 & - & - & - & - & - & - & - & - & - & - \\
\hline N II & 4630.54 & - & - & - & - & - & - & - & - & - & - \\
\hline N II & 4643.08 & - & - & - & - & - & - & - & - & - & - \\
\hline O II & 3912.00 & - & - & - & - & - & - & - & - & - & - \\
\hline O II & 3919.30 & - & - & - & - & - & - & - & - & - & - \\
\hline O II & 3945.04 & - & - & - & - & - & - & - & - & - & - \\
\hline O II & 3954.36 & - & - & - & - & - & - & - & - & - & - \\
\hline O II & 3982.71 & - & - & - & - & - & - & - & - & - & - \\
\hline O II & 4069.00 & - & - & - & - & - & - & - & - & 108 & 7.97 \\
\hline O II & 4072.15 & - & - & - & - & - & - & - & - & 69 & 7.83 \\
\hline O II & 4075.86 & - & - & - & - & - & - & - & - & - & - \\
\hline O II & 4078.84 & - & - & - & - & - & - & - & - & 33 & 8.19 \\
\hline O II & 4132.80 & - & - & - & - & - & - & - & - & - & - \\
\hline O II & 4156.53 & - & - & - & - & - & - & - & - & - & - \\
\hline O II & 4185.44 & - & - & - & - & - & - & - & - & - & - \\
\hline O II & 4317.00 & - & - & - & - & - & - & - & - & 42 & 7.92 \\
\hline O II & 4319.63 & - & - & - & - & - & - & - & - & 59 & 8.17 \\
\hline O II & 4325.76 & - & - & - & - & - & - & - & - & - & - \\
\hline O II & 4349.43 & - & - & - & - & - & - & - & - & - & - \\
\hline O II & 4351.00 & - & - & - & - & - & - & - & - & - & - \\
\hline O II & 4353.58 & - & - & - & - & - & - & - & - & - & - \\
\hline O II & 4366.00 & - & - & - & - & - & - & - & - & 56 & 8.05 \\
\hline O II & 4369.27 & - & - & - & - & - & - & - & - & - & - \\
\hline O II & 4395.94 & - & - & - & - & - & - & - & - & - & - \\
\hline O II & 4414.90 & - & - & - & - & - & - & - & - & 73 & 7.89 \\
\hline O II & 4416.98 & - & - & - & - & - & - & - & - & 56 & 7.95 \\
\hline O II & 4443.01 & - & - & - & - & - & - & - & - & - & - \\
\hline O II & 4452.38 & - & - & - & - & - & - & - & - & - & - \\
\hline O II & 4590.97 & - & - & - & - & - & - & 48 & 7.97 & 59 & 7.95 \\
\hline O II & 4596.18 & - & - & - & - & - & - & 25 & 7.62 & 50 & 7.94 \\
\hline O II & 4638.86 & - & - & - & - & - & - & 23 & 7.56 & 55 & 8.00 \\
\hline O II & 4641.81 & - & - & - & - & - & - & 52 & 7.74 & 82 & 7.89 \\
\hline O II & 4650.00 & 75 & 7.91 & 46 & 7.76 & - & - & 112 & 7.85 & 202 & 8.15 \\
\hline O II & 4661.63 & - & - & - & - & - & - & - & - & 68 & 8.09 \\
\hline O II & 4673.73 & - & - & - & - & - & - & - & - & - & - \\
\hline O II & 4676.23 & - & - & - & - & - & - & - & - & - & - \\
\hline O II & 4699.00 & - & - & - & - & - & - & - & - & 47 & 7.85 \\
\hline O II & 4705.35 & - & - & - & - & - & - & - & - & - & - \\
\hline O II & 4710.00 & - & - & - & - & - & - & - & - & - & - \\
\hline Mg II & 4481.00 & - & - & 64 & 6.56 & - & - & - & - & 58 & 6.85 \\
\hline Si II & 4128.05 & - & - & - & - & - & - & - & - & - & - \\
\hline Si II & 4130.89 & - & - & - & - & - & - & - & - & - & - \\
\hline Si III & 4552.62 & 81 & 6.78 & 60 & 6.83 & 92 & 6.80 & 73 & 6.63 & 109 & 6.71 \\
\hline Si III & 4567.84 & - & - & 45 & 6.77 & - & - & 52 & 6.52 & 94 & 6.82 \\
\hline Si III & 4574.75 & - & - & - & - & - & - & - & - & 61 & 6.97 \\
\hline Si IV & 4088.90 & - & - & - & - & - & - & - & - & - & - \\
\hline Si IV & 4116.10 & - & - & - & - & - & - & - & - & - & - \\
\hline Si IV & 4212.00 & - & - & - & - & - & - & - & - & - & - \\
\hline
\end{tabular}


I. Hunter et al.: Chemical compositions of B-type stars, Online Material p 20

Table 1. continued.

\begin{tabular}{|c|c|c|c|c|c|c|c|c|c|c|c|}
\hline \multirow[t]{2}{*}{ Species } & \multirow[t]{2}{*}{ Wavelength } & \multicolumn{2}{|c|}{ NGC 346-092 } & \multicolumn{2}{|c|}{ NGC 346-094 } & \multicolumn{2}{|c|}{ NGC 346-098 } & \multicolumn{2}{|c|}{ NGC 346-099 } & \multicolumn{2}{|c|}{ NGC 346-100 } \\
\hline & & $E W$ & Abund & $E W$ & Abund & $E W$ & Abund & $E W$ & Abund & $E W$ & Abund \\
\hline C II & 4267.00 & 73 & 7.72 & 63 & 7.68 & 76 & 7.63 & 91 & 7.13 & 72 & 7.56 \\
\hline N II & 3955.85 & - & - & - & - & - & - & - & - & - & - \\
\hline N II & 3995.00 & $<80$ & $<7.52$ & 57 & 7.34 & 38 & 6.94 & $<30$ & $<7.14$ & $<65$ & $<7.41$ \\
\hline N II & 4447.03 & - & - & - & - & - & - & - & - & - & - \\
\hline N II & 4601.48 & - & - & - & - & - & - & - & - & - & - \\
\hline N II & 4613.86 & - & - & - & - & - & - & - & - & - & - \\
\hline N II & 4630.54 & - & - & - & - & - & - & - & - & - & - \\
\hline N II & 4643.08 & - & - & - & - & - & - & - & - & - & - \\
\hline O II & 3912.00 & - & - & - & - & - & - & - & - & - & - \\
\hline O II & 3919.30 & - & - & - & - & - & - & - & - & - & - \\
\hline O II & 3945.04 & - & - & - & - & - & - & - & - & - & - \\
\hline O II & 3954.36 & - & - & 59 & 8.21 & 37 & 8.01 & - & - & - & - \\
\hline O II & 3982.71 & - & - & - & - & - & - & - & - & - & - \\
\hline O II & 4069.00 & - & - & 125 & 8.08 & 84 & 7.96 & - & - & - & - \\
\hline O II & 4072.15 & - & - & 79 & 7.93 & 51 & 7.79 & - & - & - & - \\
\hline O II & 4075.86 & - & - & 126 & 8.24 & 54 & 7.66 & - & - & - & - \\
\hline O II & 4078.84 & - & - & - & - & - & - & - & - & - & - \\
\hline O II & 4132.80 & - & - & - & - & - & - & - & - & - & - \\
\hline O II & 4156.53 & - & - & - & - & - & - & - & - & - & - \\
\hline O II & 4185.44 & - & - & - & - & - & - & - & - & - & - \\
\hline O II & 4317.00 & - & - & 69 & 8.34 & 23 & 7.63 & - & - & - & - \\
\hline O II & 4319.63 & - & - & 56 & 8.18 & 30 & 7.80 & - & - & - & - \\
\hline O II & 4325.76 & - & - & - & - & - & - & - & - & - & - \\
\hline O II & 4349.43 & - & - & 101 & 8.35 & - & - & - & - & - & - \\
\hline O II & 4351.00 & - & - & 53 & 7.80 & - & - & - & - & - & - \\
\hline O II & 4353.58 & - & - & - & - & - & - & - & - & - & - \\
\hline O II & 4366.00 & - & - & 62 & 8.18 & 32 & 7.79 & - & - & - & - \\
\hline O II & 4369.27 & - & - & - & - & - & - & - & - & - & - \\
\hline O II & 4395.94 & - & - & - & - & - & - & - & - & - & - \\
\hline O II & 4414.90 & 85 & 8.03 & 72 & 7.92 & 49 & 7.73 & - & - & - & - \\
\hline O II & 4416.98 & 73 & 8.17 & 60 & 8.04 & 53 & 8.08 & - & - & - & - \\
\hline O II & 4443.01 & - & - & - & - & - & - & - & - & - & - \\
\hline O II & 4452.38 & - & - & - & - & - & - & - & - & - & - \\
\hline O II & 4590.97 & - & - & 62 & 7.98 & 32 & 7.69 & - & - & - & - \\
\hline O II & 4596.18 & - & - & 55 & 8.00 & 21 & 7.54 & - & - & - & - \\
\hline O II & 4638.86 & 85 & 8.38 & 69 & 8.20 & 36 & 7.86 & - & - & - & - \\
\hline O II & 4641.81 & 73 & 7.81 & 96 & 8.04 & 55 & 7.78 & - & - & - & - \\
\hline O II & 4650.00 & 183 & 8.06 & - & - & 105 & 7.78 & - & - & 137 & 8.21 \\
\hline O II & 4661.63 & 66 & 8.07 & 69 & 8.12 & 35 & 7.78 & - & - & 32 & 7.83 \\
\hline O II & 4673.73 & - & - & - & - & 16 & 8.23 & - & - & - & - \\
\hline O II & 4676.23 & - & - & 55 & 8.09 & 30 & 7.79 & - & - & - & - \\
\hline O II & 4699.00 & - & - & - & - & 27 & 7.64 & - & - & - & - \\
\hline O II & 4705.35 & - & - & 77 & 8.20 & 25 & 7.59 & - & - & - & - \\
\hline O II & 4710.00 & - & - & - & - & - & - & - & - & - & - \\
\hline Mg II & 4481.00 & - & - & 46 & 6.75 & 74 & 6.99 & 100 & 6.47 & - & - \\
\hline Si II & 4128.05 & - & - & - & - & - & - & - & - & - & - \\
\hline Si II & 4130.89 & - & - & - & - & - & - & - & - & - & - \\
\hline Si III & 4552.62 & 113 & 6.80 & 109 & 6.69 & 84 & 6.70 & 49 & 6.79 & 66 & 6.57 \\
\hline Si III & 4567.84 & - & - & 99 & 6.88 & 70 & 6.75 & - & - & - & - \\
\hline Si III & 4574.75 & - & - & 47 & 6.83 & 45 & 6.86 & - & - & - & - \\
\hline Si IV & 4088.90 & - & - & - & - & - & - & - & - & - & - \\
\hline Si IV & 4116.10 & - & - & 64 & 6.78 & - & - & - & - & - & - \\
\hline Si IV & 4212.00 & - & - & - & - & - & - & - & - & - & - \\
\hline
\end{tabular}


I. Hunter et al.: Chemical compositions of B-type stars, Online Material p 21

Table 1. continued.

\begin{tabular}{|c|c|c|c|c|c|c|c|c|c|c|c|}
\hline \multirow[t]{2}{*}{ Species } & \multirow[t]{2}{*}{ Wavelength } & \multicolumn{2}{|c|}{ NGC 346-101 } & \multicolumn{2}{|c|}{ NGC 346-102 } & \multicolumn{2}{|c|}{ NGC 346-103 } & \multicolumn{2}{|c|}{ NGC 346-104 } & \multicolumn{2}{|c|}{ NGC 346-106 } \\
\hline & & $E W$ & Abund & $E W$ & Abund & $E W$ & Abund & $E W$ & Abund & $E W$ & Abund \\
\hline C II & 4267.00 & 42 & 7.33 & 50 & 6.88 & 26 & 7.23 & - & - & - & - \\
\hline N II & 3955.85 & - & - & - & - & - & - & - & - & - & - \\
\hline N II & 3995.00 & 20 & 6.63 & $<15$ & $<6.97$ & 53 & 7.48 & $<20$ & $<7.20$ & $<70$ & $<7.51$ \\
\hline N II & 4447.03 & - & - & - & - & 43 & 7.75 & - & - & - & - \\
\hline N II & 4601.48 & - & - & - & - & 19 & 7.63 & - & - & - & - \\
\hline N II & 4613.86 & - & - & - & - & 11 & 7.58 & - & - & - & - \\
\hline N II & 4630.54 & - & - & - & - & 36 & 7.48 & - & - & - & - \\
\hline N II & 4643.08 & - & - & - & - & - & - & - & - & - & - \\
\hline O II & 3912.00 & - & - & - & - & - & - & - & - & - & - \\
\hline O II & 3919.30 & - & - & - & - & - & - & - & - & - & - \\
\hline O II & 3945.04 & - & - & - & - & 16 & 7.88 & - & - & - & - \\
\hline O II & 3954.36 & 17 & 7.54 & - & - & 35 & 8.00 & - & - & - & - \\
\hline O II & 3982.71 & - & - & - & - & - & - & - & - & - & - \\
\hline O II & 4069.00 & 41 & 7.39 & - & - & 93 & 8.00 & - & - & - & - \\
\hline O II & 4072.15 & 35 & 7.45 & - & - & 49 & 7.71 & 24 & 7.46 & - & - \\
\hline O II & 4075.86 & 36 & 7.30 & - & - & 92 & 8.20 & - & - & - & - \\
\hline O II & 4078.84 & 11 & 7.59 & - & - & - & - & - & - & - & - \\
\hline O II & 4132.80 & - & - & - & - & - & - & - & - & - & - \\
\hline O II & 4156.53 & - & - & - & - & - & - & - & - & - & - \\
\hline O II & 4185.44 & - & - & - & - & - & - & - & - & - & - \\
\hline O II & 4317.00 & - & - & - & - & 35 & 8.02 & 15 & 7.87 & - & - \\
\hline O II & 4319.63 & - & - & - & - & 45 & 8.19 & 14 & 7.85 & - & - \\
\hline O II & 4325.76 & - & - & - & - & - & - & - & - & - & - \\
\hline O II & 4349.43 & - & - & - & - & 61 & 8.14 & - & - & - & - \\
\hline O II & 4351.00 & - & - & - & - & 39 & 7.68 & - & - & - & - \\
\hline O II & 4353.58 & - & - & - & - & - & - & - & - & - & - \\
\hline O II & 4366.00 & - & - & - & - & 30 & 7.87 & 25 & 8.06 & - & - \\
\hline O II & 4369.27 & - & - & - & - & - & - & - & - & - & - \\
\hline O II & 4395.94 & - & - & - & - & - & - & - & - & - & - \\
\hline O II & 4414.90 & 38 & 7.54 & - & - & 52 & 7.86 & - & - & 60 & 7.87 \\
\hline O II & 4416.98 & - & - & - & - & 47 & 8.05 & - & - & 50 & 8.00 \\
\hline O II & 4443.01 & - & - & - & - & - & - & - & - & - & - \\
\hline O II & 4452.38 & - & - & - & - & - & - & - & - & - & - \\
\hline O II & 4590.97 & 18 & 7.34 & - & - & 45 & 7.88 & 16 & 7.54 & - & - \\
\hline O II & 4596.18 & 20 & 7.50 & - & - & 48 & 8.04 & 11 & 7.49 & - & - \\
\hline O II & 4638.86 & 22 & 7.54 & - & - & 39 & 7.95 & 22 & 7.94 & 47 & 8.04 \\
\hline O II & 4641.81 & 42 & 7.57 & - & - & 86 & 8.20 & 12 & 7.37 & 74 & 8.05 \\
\hline O II & 4650.00 & 85 & 7.61 & 31 & 8.41 & 134 & 8.01 & 70 & 7.86 & 200 & 8.50 \\
\hline O II & 4661.63 & 31 & 7.69 & - & - & 43 & 7.96 & 13 & 7.64 & - & - \\
\hline O II & 4673.73 & - & - & - & - & - & - & - & - & - & - \\
\hline O II & 4676.23 & - & - & - & - & 36 & 7.97 & 19 & 7.91 & - & - \\
\hline O II & 4699.00 & 20 & 7.46 & - & - & 35 & 7.80 & - & - & - & - \\
\hline O II & 4705.35 & - & - & - & - & 36 & 7.82 & - & - & - & - \\
\hline O II & 4710.00 & - & - & - & - & - & - & - & - & - & - \\
\hline Mg II & 4481.00 & 31 & 6.49 & - & - & 44 & 6.82 & 21 & 6.51 & - & - \\
\hline Si II & 4128.05 & - & - & - & - & - & - & - & - & - & - \\
\hline Si II & 4130.89 & - & - & - & - & - & - & - & - & - & - \\
\hline Si III & 4552.62 & 55 & 6.31 & 27 & 6.77 & 80 & 6.80 & 45 & 6.62 & 66 & 6.50 \\
\hline Si III & 4567.84 & 51 & 6.50 & 22 & 6.85 & 59 & 6.74 & 30 & 6.55 & - & - \\
\hline Si III & 4574.75 & 25 & 6.47 & - & - & 34 & 6.85 & 10 & 6.43 & - & - \\
\hline Si IV & 4088.90 & - & - & - & - & - & - & - & - & - & - \\
\hline Si IV & 4116.10 & - & - & - & - & 54 & 6.68 & 56 & 6.56 & - & - \\
\hline Si IV & 4212.00 & - & - & - & - & 12 & 6.80 & - & - & - & - \\
\hline
\end{tabular}


I. Hunter et al.: Chemical compositions of B-type stars, Online Material p 22

Table 1. continued.

\begin{tabular}{|c|c|c|c|c|c|c|c|c|c|c|c|}
\hline \multirow[t]{2}{*}{ Species } & \multirow[t]{2}{*}{ Wavelength } & \multicolumn{2}{|c|}{ NGC 346-108 } & \multicolumn{2}{|c|}{ NGC 346-109 } & \multicolumn{2}{|c|}{ NGC 346-114 } & \multicolumn{2}{|c|}{ NGC 346-116 } & \multicolumn{2}{|c|}{ NGC 330-002 } \\
\hline & & $E W$ & Abund & $E W$ & Abund & $E W$ & Abund & $E W$ & Abund & $E W$ & Abund \\
\hline C II & 4267.00 & 70 & 7.59 & 89 & 7.70 & - & - & 43 & 7.43 & 82 & 6.91 \\
\hline N II & 3955.85 & - & - & - & - & - & - & - & - & - & - \\
\hline N II & 3995.00 & $<80$ & $<7.42$ & $<50$ & $<7.09$ & $<110$ & $<7.80$ & 28 & 6.93 & 56 & 7.49 \\
\hline N II & 4447.03 & - & - & - & - & - & - & _- & - & 23 & 7.70 \\
\hline N II & 4601.48 & - & - & - & - & - & - & - & - & 18 & 7.62 \\
\hline N II & 4613.86 & - & - & - & - & - & - & - & - & 11 & 7.59 \\
\hline N II & 4630.54 & - & - & - & - & - & - & - & - & 36 & 7.55 \\
\hline N II & 4643.08 & - & - & - & - & - & - & - & - & 18 & 7.63 \\
\hline O II & 3912.00 & - & - & - & - & - & - & 54 & 8.23 & - & - \\
\hline O II & 3919.30 & - & - & - & - & - & - & - & - & - & - \\
\hline O II & 3945.04 & - & - & - & - & - & - & 17 & 7.86 & - & - \\
\hline O II & 3954.36 & - & - & - & - & - & - & 46 & 8.15 & 14 & 8.34 \\
\hline O II & 3982.71 & - & - & - & - & - & - & - & - & - & - \\
\hline O II & 4069.00 & - & - & - & - & - & - & 136 & 8.41 & 11 & 7.86 \\
\hline O II & 4072.15 & - & - & - & - & - & - & 68 & 8.05 & 13 & 8.01 \\
\hline O II & 4075.86 & - & - & 58 & 7.69 & - & - & - & - & 15 & 7.91 \\
\hline O II & 4078.84 & - & - & - & - & - & - & - & - & 3 & 7.99 \\
\hline O II & 4132.80 & - & - & - & - & - & - & 34 & 8.13 & - & - \\
\hline O II & 4156.53 & - & - & - & - & - & - & - & - & - & - \\
\hline O II & 4185.44 & - & - & - & - & - & - & 45 & 8.17 & - & - \\
\hline O II & 4317.00 & - & - & 34 & 7.91 & - & - & 44 & 8.10 & 8 & 8.06 \\
\hline O II & 4319.63 & - & - & 32 & 7.87 & - & - & 52 & 8.25 & 10 & 8.15 \\
\hline O II & 4325.76 & - & - & - & - & - & - & - & - & - & - \\
\hline O II & 4349.43 & - & - & - & - & - & - & 73 & 8.29 & 10 & 7.86 \\
\hline O II & 4351.00 & - & - & - & - & - & - & 48 & 7.87 & - & - \\
\hline O II & 4353.58 & - & - & - & - & - & - & - & - & - & - \\
\hline O II & 4366.00 & - & - & - & - & - & - & 41 & 7.99 & - & - \\
\hline O II & 4369.27 & - & - & - & - & - & - & - & - & - & - \\
\hline O II & 4395.94 & - & - & - & - & - & - & - & - & - & - \\
\hline O II & 4414.90 & - & - & - & - & - & - & 77 & 8.12 & 12 & 7.86 \\
\hline O II & 4416.98 & - & - & - & - & - & - & 59 & 8.16 & 12 & 8.06 \\
\hline O II & 4443.01 & - & - & - & - & - & - & - & - & - & - \\
\hline O II & 4452.38 & - & - & - & - & - & - & 35 & 8.49 & - & - \\
\hline O II & 4590.97 & 60 & 8.03 & - & - & - & - & 58 & 8.10 & - & - \\
\hline O II & 4596.18 & 51 & 8.01 & - & - & - & - & 53 & 8.10 & - & - \\
\hline O II & 4638.86 & - & - & - & - & - & - & 56 & 8.18 & 5 & 7.81 \\
\hline O II & 4641.81 & - & - & - & - & - & - & 85 & 8.20 & 13 & 7.88 \\
\hline O II & 4650.00 & 138 & 7.85 & 118 & 7.84 & - & - & 162 & 8.19 & - & - \\
\hline O II & 4661.63 & - & - & - & - & - & - & 55 & 8.11 & 7 & 7.93 \\
\hline O II & 4673.73 & - & - & - & - & - & - & - & - & - & - \\
\hline O II & 4676.23 & - & - & - & - & - & - & 51 & 8.16 & 7 & 8.06 \\
\hline O II & 4699.00 & - & - & - & - & - & - & 45 & 7.88 & - & - \\
\hline O II & 4705.35 & - & - & - & - & - & - & 45 & 7.93 & - & - \\
\hline O II & 4710.00 & - & - & - & - & - & - & - & - & - & - \\
\hline $\mathrm{Mg}$ II & 4481.00 & 59 & 6.78 & - & - & - & - & 40 & 6.70 & 174 & 6.60 \\
\hline Si II & 4128.05 & - & - & - & - & - & - & - & - & 75 & 6.93 \\
\hline Si II & 4130.89 & - & - & - & - & - & - & - & - & 84 & 6.81 \\
\hline Si III & 4552.62 & 92 & 6.47 & 111 & 6.79 & 118 & 6.77 & 78 & 6.76 & 66 & 6.84 \\
\hline Si III & 4567.84 & 118 & 7.04 & 74 & 6.62 & - & - & 66 & 6.82 & 46 & 6.82 \\
\hline Si III & 4574.75 & - & - & 54 & 6.89 & - & - & 38 & 6.86 & 20 & 6.79 \\
\hline Si IV & 4088.90 & - & - & - & - & - & - & - & - & - & - \\
\hline Si IV & 4116.10 & - & - & - & - & - & - & 36 & 6.75 & - & - \\
\hline Si IV & 4212.00 & - & - & - & - & - & - & - & - & - & - \\
\hline
\end{tabular}


I. Hunter et al.: Chemical compositions of B-type stars, Online Material p 23

Table 1. continued.

\begin{tabular}{|c|c|c|c|c|c|c|c|c|c|c|c|}
\hline \multirow[t]{2}{*}{ Species } & \multirow[t]{2}{*}{ Wavelength } & \multicolumn{2}{|c|}{ NGC 330-003 } & \multicolumn{2}{|c|}{ NGC 330-004 } & \multicolumn{2}{|c|}{ NGC 330-005 } & \multicolumn{2}{|c|}{ NGC 330-009 } & \multicolumn{2}{|c|}{ NGC 330-010 } \\
\hline & & $E W$ & Abund & $E W$ & Abund & $E W$ & Abund & $E W$ & Abund & $E W$ & Abund \\
\hline C II & 4267.00 & 134 & 7.14 & 62 & 6.72 & 32 & 6.59 & 48 & 6.87 & 64 & 6.99 \\
\hline N II & 3955.85 & - & - & - & - & - & - & - & - & - & - \\
\hline N II & 3995.00 & 150 & 7.62 & 146 & 7.75 & 24 & 7.41 & 16 & 7.19 & 15 & 7.06 \\
\hline N II & 4447.03 & 67 & 7.74 & 64 & 7.80 & - & - & - & - & - & - \\
\hline N II & 4601.48 & 51 & 7.67 & 63 & 7.86 & 6 & 7.51 & - & - & - & - \\
\hline N II & 4613.86 & 33 & 7.67 & 41 & 7.85 & - & - & - & - & - & - \\
\hline $\mathrm{N}$ II & 4630.54 & 117 & 7.67 & 126 & 7.86 & 13 & 7.42 & 8 & 7.21 & 10 & 7.21 \\
\hline N II & 4643.08 & 65 & 7.80 & 69 & 7.90 & - & - & - & - & - & - \\
\hline O II & 3912.00 & 27 & 8.12 & - & - & - & - & - & - & - & - \\
\hline O II & 3919.30 & - & - & - & - & - & - & - & - & - & - \\
\hline O II & 3945.04 & 19 & 8.13 & - & - & - & - & - & - & - & - \\
\hline O II & 3954.36 & 17 & 7.78 & 15 & 7.79 & - & - & - & - & - & - \\
\hline O II & 3982.71 & - & - & - & - & - & - & - & - & - & - \\
\hline O II & 4069.00 & 61 & 7.98 & 36 & 7.81 & - & - & 6 & 8.05 & - & - \\
\hline O II & 4072.15 & 56 & 7.99 & 31 & 7.81 & - & - & 9 & 8.44 & 4 & 7.75 \\
\hline O II & 4075.86 & 69 & 7.97 & 37 & 7.75 & 4 & 7.83 & 11 & 8.42 & 4 & 7.69 \\
\hline O II & 4078.84 & 8 & 7.78 & - & - & - & - & - & - & - & - \\
\hline O II & 4132.80 & - & - & - & - & - & - & - & - & - & - \\
\hline O II & 4156.53 & - & - & - & - & - & - & - & - & - & - \\
\hline O II & 4185.44 & - & - & - & - & - & - & - & - & - & - \\
\hline O II & 4317.00 & 43 & 8.26 & 19 & 7.89 & - & - & - & - & - & - \\
\hline O II & 4319.63 & 45 & 8.30 & 22 & 7.98 & - & - & - & - & - & - \\
\hline O II & 4325.76 & 6 & 7.96 & - & - & - & - & - & - & - & - \\
\hline O II & 4349.43 & 71 & 8.22 & 34 & 7.88 & - & - & - & - & - & - \\
\hline O II & 4351.00 & 21 & 7.83 & - & - & - & - & - & - & - & - \\
\hline O II & 4353.58 & - & - & - & - & - & - & - & - & - & - \\
\hline O II & 4366.00 & 46 & 8.23 & 18 & 7.82 & - & - & - & - & - & - \\
\hline O II & 4369.27 & - & - & - & - & - & - & - & - & - & - \\
\hline O II & 4395.94 & - & - & - & - & - & - & - & - & - & - \\
\hline O II & 4414.90 & 70 & 8.10 & 41 & 7.89 & - & - & - & - & - & - \\
\hline O II & 4416.98 & 48 & 8.11 & 30 & 7.95 & - & - & - & - & - & - \\
\hline O II & 4443.01 & - & - & - & - & - & - & - & - & - & - \\
\hline O II & 4452.38 & - & - & - & - & - & - & - & - & - & - \\
\hline O II & 4590.97 & 33 & 8.00 & - & - & - & - & - & - & - & - \\
\hline O II & 4596.18 & 25 & 7.98 & - & - & - & - & - & - & - & - \\
\hline O II & 4638.86 & 42 & 8.15 & 19 & 7.83 & - & - & - & - & - & - \\
\hline O II & 4641.81 & 82 & 8.14 & 40 & 7.84 & - & - & - & - & - & - \\
\hline O II & 4650.00 & 173 & 8.23 & 79 & 7.87 & 8 & 7.82 & - & - & 8 & 7.70 \\
\hline O II & 4661.63 & 50 & 8.17 & 24 & 7.89 & - & - & - & - & - & - \\
\hline O II & 4673.73 & - & - & - & - & - & - & - & - & - & - \\
\hline O II & 4676.23 & 43 & 8.21 & 16 & 7.83 & - & - & - & - & - & - \\
\hline O II & 4699.00 & 16 & 7.87 & - & - & - & - & - & - & - & - \\
\hline O II & 4705.35 & 26 & 8.06 & - & - & - & - & - & - & - & - \\
\hline O II & 4710.00 & - & - & - & - & - & - & - & - & - & - \\
\hline Mg II & 4481.00 & 131 & 6.79 & 128 & 6.73 & 192 & 6.58 & 202 & 6.66 & 160 & 6.63 \\
\hline Si II & 4128.05 & 40 & 6.91 & 40 & 6.87 & 73 & 6.90 & 68 & 6.93 & 53 & 6.97 \\
\hline Si II & 4130.89 & 52 & 6.86 & 47 & 6.77 & 77 & 6.74 & 74 & 6.80 & 56 & 6.78 \\
\hline Si III & 4552.62 & 184 & 6.77 & 149 & 6.82 & 26 & 6.82 & 24 & 6.86 & 28 & 6.90 \\
\hline Si III & 4567.84 & 146 & 6.81 & 113 & 6.82 & 18 & 6.79 & 15 & 6.76 & 19 & 6.84 \\
\hline Si III & 4574.75 & 76 & 6.85 & 56 & 6.81 & 8 & 6.81 & - & - & 8 & 6.79 \\
\hline Si IV & 4088.90 & - & - & - & - & - & - & - & - & - & - \\
\hline Si IV & 4116.10 & - & - & - & - & - & - & - & - & - & - \\
\hline Si IV & 4212.00 & - & - & - & - & - & - & - & - & - & - \\
\hline
\end{tabular}


I. Hunter et al.: Chemical compositions of B-type stars, Online Material p 24

Table 1. continued.

\begin{tabular}{|c|c|c|c|c|c|c|c|c|c|c|c|}
\hline \multirow[t]{2}{*}{ Species } & \multirow[t]{2}{*}{ Wavelength } & \multicolumn{2}{|c|}{ NGC 330-014 } & \multicolumn{2}{|c|}{ NGC 330-016 } & \multicolumn{2}{|c|}{ NGC 330-017 } & \multicolumn{2}{|c|}{ NGC 330-018 } & \multicolumn{2}{|c|}{ NGC 330-020 } \\
\hline & & $E W$ & Abund & $E W$ & Abund & $E W$ & Abund & $E W$ & Abund & $E W$ & Abund \\
\hline C II & 4267.00 & 66 & 7.02 & 69 & 7.18 & 74 & 7.30 & 102 & 7.10 & 76 & 6.92 \\
\hline N II & 3955.85 & - & - & - & - & - & - & - & - & - & - \\
\hline N II & 3995.00 & 157 & 7.47 & 15 & 7.18 & 48 & 7.04 & 50 & 7.31 & 22 & 6.93 \\
\hline N II & 4447.03 & 69 & 7.54 & - & - & 24 & 7.12 & - & - & - & - \\
\hline N II & 4601.48 & 64 & 7.61 & - & - & 20 & 7.18 & - & - & - & - \\
\hline N II & 4613.86 & 35 & 7.52 & - & - & 16 & 7.28 & - & - & - & - \\
\hline N II & 4630.54 & 112 & 7.46 & - & - & 39 & 7.12 & 26 & 7.18 & 15 & 7.10 \\
\hline N II & 4643.08 & - & - & - & - & 20 & 7.15 & - & - & - & - \\
\hline O II & 3912.00 & 39 & 8.00 & - & - & 28 & 7.99 & - & - & - & - \\
\hline O II & 3919.30 & - & - & - & - & - & - & - & - & - & - \\
\hline O II & 3945.04 & 37 & 8.21 & - & - & 11 & 7.70 & - & - & - & - \\
\hline O II & 3954.36 & - & - & - & - & 20 & 7.75 & - & - & - & - \\
\hline O II & 3982.71 & - & - & - & - & - & - & - & - & - & - \\
\hline O II & 4069.00 & 129 & 8.16 & 4 & 7.89 & 53 & 7.84 & 34 & 8.13 & 17 & 7.96 \\
\hline O II & 4072.15 & 101 & 8.06 & - & - & 26 & 7.50 & 21 & 7.99 & 11 & 7.85 \\
\hline O II & 4075.86 & 114 & 7.98 & 8 & 8.25 & 35 & 7.58 & 30 & 8.09 & 13 & 7.84 \\
\hline O II & 4078.84 & 24 & 8.02 & - & - & 11 & 7.79 & - & - & - & - \\
\hline O II & 4132.80 & 30 & 8.02 & - & - & 15 & 7.82 & - & - & - & - \\
\hline O II & 4156.53 & - & - & - & - & - & - & - & - & - & - \\
\hline O II & 4185.44 & - & - & - & - & - & - & - & - & - & - \\
\hline O II & 4317.00 & 70 & 8.25 & - & - & 17 & 7.56 & - & - & - & - \\
\hline O II & 4319.63 & 78 & 8.32 & - & - & 22 & 7.75 & - & - & - & - \\
\hline O II & 4325.76 & - & - & - & - & - & - & - & - & - & - \\
\hline O II & 4349.43 & - & - & - & - & 33 & 7.75 & 18 & 7.90 & - & - \\
\hline O II & 4351.00 & - & - & - & - & 18 & 7.59 & - & - & - & - \\
\hline O II & 4353.58 & - & - & - & - & - & - & - & - & - & - \\
\hline O II & 4366.00 & 70 & 8.17 & - & - & 17 & 7.51 & - & - & - & - \\
\hline O II & 4369.27 & - & - & - & - & - & - & - & - & - & - \\
\hline O II & 4395.94 & - & - & - & - & - & - & - & - & - & - \\
\hline O II & 4414.90 & 98 & 8.02 & - & - & 25 & 7.41 & 27 & 8.05 & - & - \\
\hline O II & 4416.98 & 62 & 7.96 & - & - & 21 & 7.60 & 24 & 8.26 & - & - \\
\hline O II & 4443.01 & - & - & - & - & - & - & - & - & - & - \\
\hline O II & 4452.38 & 33 & 8.36 & - & - & - & - & - & - & - & - \\
\hline O II & 4590.97 & 70 & 8.11 & - & - & 15 & 7.45 & - & - & - & - \\
\hline O II & 4596.18 & 62 & 8.14 & - & - & 17 & 7.63 & - & - & - & - \\
\hline O II & 4638.86 & 81 & 8.24 & - & - & 20 & 7.63 & - & - & - & - \\
\hline O II & 4641.81 & - & - & - & - & 34 & 7.59 & - & - & - & - \\
\hline O II & 4650.00 & - & - & - & - & 60 & 7.51 & 45 & 7.94 & 15 & 7.55 \\
\hline O II & 4661.63 & 85 & 8.20 & - & - & 20 & 7.56 & - & - & - & - \\
\hline O II & 4673.73 & - & - & - & - & - & - & - & - & - & - \\
\hline O II & 4676.23 & 69 & 8.19 & - & - & 20 & 7.69 & - & - & - & - \\
\hline O II & 4699.00 & 30 & 7.84 & - & - & 13 & 7.45 & - & - & - & - \\
\hline O II & 4705.35 & 43 & 7.97 & - & - & 17 & 7.60 & - & - & - & - \\
\hline O II & 4710.00 & - & - & - & - & - & - & - & - & - & - \\
\hline Mg II & 4481.00 & 92 & 6.72 & 199 & 6.69 & 46 & 6.47 & 107 & 6.61 & 133 & 6.64 \\
\hline Si II & 4128.05 & - & - & 63 & 6.96 & - & - & 31 & 6.82 & 46 & 6.99 \\
\hline Si II & 4130.89 & - & - & 65 & 6.75 & - & - & 41 & 6.80 & 47 & 6.78 \\
\hline Si III & 4552.62 & 247 & 6.78 & 22 & 6.84 & 77 & 6.75 & 63 & 6.81 & 44 & 6.77 \\
\hline Si III & 4567.84 & 199 & 6.83 & 14 & 6.78 & 61 & 6.72 & 48 & 6.80 & 36 & 6.85 \\
\hline Si III & 4574.75 & 107 & 6.88 & - & - & 36 & 6.74 & 25 & 6.80 & 19 & 6.88 \\
\hline Si IV & 4088.90 & - & - & - & - & - & - & - & - & - & - \\
\hline Si IV & 4116.10 & 28 & 6.83 & - & - & - & - & - & - & - & - \\
\hline Si IV & 4212.00 & - & - & - & - & - & - & - & - & - & - \\
\hline
\end{tabular}


I. Hunter et al.: Chemical compositions of B-type stars, Online Material p 25

Table 1. continued.

\begin{tabular}{|c|c|c|c|c|c|c|c|c|c|c|c|}
\hline \multirow[t]{2}{*}{ Species } & \multirow[t]{2}{*}{ Wavelength } & \multicolumn{2}{|c|}{ NGC 330-021 } & \multicolumn{2}{|c|}{ NGC 330-022 } & \multicolumn{2}{|c|}{ NGC 330-024 } & \multicolumn{2}{|c|}{ NGC 330-026 } & \multicolumn{2}{|c|}{ NGC 330-027 } \\
\hline & & $E W$ & Abund & $E W$ & Abund & $E W$ & Abund & $E W$ & Abund & $E W$ & Abund \\
\hline C II & 4267.00 & - & - & 94 & 7.11 & 53 & 7.09 & 97 & 7.57 & 81 & 7.36 \\
\hline N II & 3955.85 & - & - & - & - & - & - & - & - & - & - \\
\hline N II & 3995.00 & - & - & 50 & 7.30 & $<20$ & $<7.53$ & 77 & 7.45 & 100 & 7.38 \\
\hline N II & 4447.03 & - & - & 16 & 7.12 & - & - & - & - & - & - \\
\hline N II & 4601.48 & - & - & 17 & 7.30 & - & - & 22 & 7.22 & - & - \\
\hline N II & 4613.86 & - & - & 13 & 7.39 & - & - & - & - & - & - \\
\hline N II & 4630.54 & $<38$ & $<7.64$ & 31 & 7.23 & - & - & 34 & 7.01 & 81 & 7.43 \\
\hline N II & 4643.08 & - & - & 19 & 7.35 & - & - & - & - & - & - \\
\hline O II & 3912.00 & - & - & - & - & - & - & - & - & - & - \\
\hline O II & 3919.30 & - & - & - & - & - & - & - & - & - & - \\
\hline O II & 3945.04 & - & - & - & - & - & - & - & - & - & - \\
\hline O II & 3954.36 & - & - & - & - & - & - & - & - & - & - \\
\hline O II & 3982.71 & - & - & - & - & - & - & - & - & - & - \\
\hline O II & 4069.00 & - & - & 25 & 7.74 & - & - & 43 & 7.61 & 140 & 8.41 \\
\hline O II & 4072.15 & - & - & 20 & 7.78 & - & - & 32 & 7.60 & 94 & 8.30 \\
\hline O II & 4075.86 & - & - & 27 & 7.88 & - & - & 43 & 7.70 & 104 & 8.23 \\
\hline O II & 4078.84 & - & - & - & - & - & - & - & - & - & - \\
\hline O II & 4132.80 & - & - & - & - & - & - & - & - & - & - \\
\hline O II & 4156.53 & - & - & - & - & - & - & - & - & - & - \\
\hline O II & 4185.44 & - & - & - & - & - & - & - & - & - & - \\
\hline O II & 4317.00 & - & - & - & - & - & - & - & - & - & - \\
\hline O II & 4319.63 & - & - & - & - & - & - & - & - & - & - \\
\hline O II & 4325.76 & - & - & - & - & - & - & - & - & - & - \\
\hline O II & 4349.43 & - & - & 17 & 7.71 & - & - & - & - & - & - \\
\hline O II & 4351.00 & - & - & - & - & - & - & - & - & - & - \\
\hline O II & 4353.58 & - & - & - & - & - & - & - & - & - & - \\
\hline O II & 4366.00 & - & - & 13 & 7.78 & - & - & - & - & - & - \\
\hline O II & 4369.27 & - & - & - & - & - & - & - & - & - & - \\
\hline O II & 4395.94 & - & - & - & - & - & - & - & - & - & - \\
\hline O II & 4414.90 & - & - & - & - & - & - & - & - & - & - \\
\hline O II & 4416.98 & - & - & - & - & - & - & - & - & - & - \\
\hline O II & 4443.01 & - & - & - & - & - & - & - & - & - & - \\
\hline O II & 4452.38 & - & - & - & - & - & - & - & - & - & - \\
\hline O II & 4590.97 & - & - & - & - & - & - & - & - & 53 & 8.02 \\
\hline O II & 4596.18 & - & - & - & - & - & - & - & - & 52 & 8.10 \\
\hline O II & 4638.86 & - & - & - & - & - & - & 38 & 8.02 & - & - \\
\hline O II & 4641.81 & - & - & 20 & 7.69 & - & - & - & - & - & - \\
\hline O II & 4650.00 & - & - & 40 & 7.70 & - & - & 86 & 7.74 & 217 & 8.28 \\
\hline O II & 4661.63 & - & - & 13 & 7.76 & - & - & 29 & 7.76 & 78 & 8.29 \\
\hline O II & 4673.73 & - & - & - & - & - & - & - & - & - & - \\
\hline O II & 4676.23 & - & - & - & - & - & - & - & - & 87 & 8.52 \\
\hline O II & 4699.00 & - & - & - & - & - & - & - & - & - & - \\
\hline O II & 4705.35 & - & - & - & - & - & - & - & - & - & - \\
\hline O II & 4710.00 & - & - & - & - & - & - & - & - & - & - \\
\hline Mg II & 4481.00 & 51 & 6.91 & 110 & 6.85 & 169 & 6.52 & 92 & 7.02 & 46 & 6.44 \\
\hline Si II & 4128.05 & - & - & 24 & 6.90 & 54 & 6.81 & - & - & - & - \\
\hline Si II & 4130.89 & - & - & 30 & 6.86 & 60 & 6.69 & - & - & - & - \\
\hline Si III & 4552.62 & 134 & 6.93 & 66 & 6.95 & - & - & 73 & 6.62 & 145 & 6.76 \\
\hline Si III & 4567.84 & - & - & 46 & 6.79 & - & - & 58 & 6.61 & 123 & 6.84 \\
\hline Si III & 4574.75 & - & - & 24 & 6.74 & - & - & 37 & 6.70 & 68 & 6.83 \\
\hline Si IV & 4088.90 & - & - & - & - & - & - & - & - & - & - \\
\hline Si IV & 4116.10 & - & - & - & - & - & - & - & - & 23 & 6.82 \\
\hline Si IV & 4212.00 & - & - & - & - & - & - & - & - & - & - \\
\hline
\end{tabular}


I. Hunter et al.: Chemical compositions of B-type stars, Online Material p 26

Table 1. continued.

\begin{tabular}{|c|c|c|c|c|c|c|c|c|c|c|c|}
\hline \multirow[t]{2}{*}{ Species } & \multirow[t]{2}{*}{ Wavelength } & \multicolumn{2}{|c|}{ NGC 330-028 } & \multicolumn{2}{|c|}{ NGC 330-032 } & \multicolumn{2}{|c|}{ NGC 330-033 } & \multicolumn{2}{|c|}{ NGC 330-035 } & \multicolumn{2}{|c|}{ NGC 330-036 } \\
\hline & & $E W$ & Abund & $E W$ & Abund & $E W$ & Abund & $E W$ & Abund & $E W$ & Abund \\
\hline C II & 4267.00 & 88 & 7.84 & 33 & 7.37 & 86 & 7.74 & 70 & 6.94 & 75 & 7.18 \\
\hline N II & 3955.85 & - & - & - & - & - & - & - & - & - & - \\
\hline N II & 3995.00 & 76 & 7.44 & 43 & 7.33 & 44 & 7.07 & 39 & 7.19 & 65 & 7.12 \\
\hline N II & 4447.03 & - & - & 24 & 7.38 & - & - & - & - & - & - \\
\hline N II & 4601.48 & - & - & - & - & - & - & - & - & - & - \\
\hline N II & 4613.86 & - & - & - & - & - & - & - & - & - & - \\
\hline N II & 4630.54 & 63 & 7.57 & 31 & 7.40 & - & - & 31 & 7.33 & 52 & 7.22 \\
\hline N II & 4643.08 & - & - & - & - & - & - & - & - & - & - \\
\hline O II & 3912.00 & 65 & 8.17 & 25 & 7.78 & - & - & - & - & - & - \\
\hline O II & 3919.30 & - & - & - & - & - & - & - & - & - & - \\
\hline O II & 3945.04 & 64 & 8.49 & - & - & - & - & - & - & - & - \\
\hline O II & 3954.36 & 48 & 7.98 & 30 & 7.92 & - & - & - & - & 37 & 8.11 \\
\hline O II & 3982.71 & - & - & - & - & - & - & - & - & - & - \\
\hline O II & 4069.00 & 116 & 7.93 & 40 & 7.42 & - & - & 27 & 7.92 & 61 & 7.89 \\
\hline O II & 4072.15 & 80 & 7.83 & 52 & 7.80 & - & - & - & - & 40 & 7.77 \\
\hline O II & 4075.86 & 87 & 7.73 & 71 & 7.93 & - & - & 31 & 8.16 & 45 & 7.69 \\
\hline O II & 4078.84 & 19 & 7.79 & 30 & 8.29 & - & - & - & - & - & - \\
\hline O II & 4132.80 & - & - & 15 & 7.66 & - & - & - & - & - & - \\
\hline O II & 4156.53 & - & - & - & - & - & - & - & - & - & - \\
\hline O II & 4185.44 & - & - & - & - & - & - & - & - & - & - \\
\hline O II & 4317.00 & 52 & 8.03 & 35 & 8.00 & 21 & 7.55 & - & - & - & - \\
\hline O II & 4319.63 & 47 & 7.96 & 40 & 8.09 & 28 & 7.73 & - & - & 27 & 7.86 \\
\hline O II & 4325.76 & - & - & - & - & - & - & - & - & - & - \\
\hline O II & 4349.43 & - & - & 65 & 8.24 & - & - & - & - & - & - \\
\hline O II & 4351.00 & - & - & 38 & 7.70 & - & - & - & - & - & - \\
\hline O II & 4353.58 & - & - & - & - & - & - & - & - & - & - \\
\hline O II & 4366.00 & 59 & 8.03 & 36 & 7.98 & 29 & 7.71 & - & - & 23 & 7.69 \\
\hline O II & 4369.27 & - & - & - & - & - & - & - & - & - & - \\
\hline O II & 4395.94 & - & - & - & - & - & - & - & - & 18 & 8.26 \\
\hline O II & 4414.90 & 85 & 7.94 & 56 & 7.91 & - & - & - & - & 48 & 7.84 \\
\hline O II & 4416.98 & 73 & 8.08 & 46 & 8.04 & - & - & - & - & 40 & 7.97 \\
\hline O II & 4443.01 & - & - & - & - & - & - & - & - & - & - \\
\hline O II & 4452.38 & - & - & - & - & - & - & - & - & - & - \\
\hline O II & 4590.97 & 54 & 7.79 & 35 & 7.74 & - & - & - & - & 32 & 7.91 \\
\hline O II & 4596.18 & 53 & 7.89 & 33 & 7.82 & - & - & - & - & 35 & 8.07 \\
\hline O II & 4638.86 & 62 & 8.01 & 41 & 8.00 & - & - & - & - & 36 & 8.00 \\
\hline O II & 4641.81 & 92 & 7.88 & 64 & 7.95 & - & - & - & - & 52 & 7.84 \\
\hline O II & 4650.00 & - & - & - & - & - & - & - & - & 111 & 7.94 \\
\hline O II & 4661.63 & 61 & 7.93 & 46 & 8.02 & - & - & - & - & 40 & 8.00 \\
\hline O II & 4673.73 & 32 & 8.47 & - & - & - & - & - & - & - & - \\
\hline O II & 4676.23 & 67 & 8.12 & 38 & 8.00 & - & - & - & - & 32 & 7.97 \\
\hline O II & 4699.00 & 53 & 7.90 & 37 & 7.82 & - & - & - & - & 26 & 7.88 \\
\hline O II & 4705.35 & 51 & 7.87 & 35 & 7.79 & - & - & - & - & 32 & 8.02 \\
\hline O II & 4710.00 & - & - & - & - & - & - & - & - & - & - \\
\hline Mg II & 4481.00 & 59 & 6.86 & 41 & 6.80 & 96 & 7.26 & 89 & 6.68 & 55 & 6.45 \\
\hline Si II & 4128.05 & - & - & - & - & - & - & - & - & - & - \\
\hline Si II & 4130.89 & - & - & - & - & - & - & - & - & - & - \\
\hline Si III & 4552.62 & 119 & 6.57 & 65 & 6.65 & 70 & 6.52 & 44 & 6.68 & 111 & 6.72 \\
\hline Si III & 4567.84 & 106 & 6.75 & 59 & 6.82 & 73 & 6.85 & 38 & 6.78 & 84 & 6.69 \\
\hline Si III & 4574.75 & 79 & 7.08 & 31 & 6.84 & 49 & 6.97 & 18 & 6.63 & 63 & 6.96 \\
\hline Si IV & 4088.90 & - & - & - & - & - & - & - & - & - & - \\
\hline Si IV & 4116.10 & - & - & 49 & 6.77 & - & - & - & - & - & - \\
\hline Si IV & 4212.00 & - & - & - & - & - & - & - & - & - & - \\
\hline
\end{tabular}


I. Hunter et al.: Chemical compositions of B-type stars, Online Material p 27

Table 1. continued.

\begin{tabular}{|c|c|c|c|c|c|c|c|c|c|c|c|}
\hline \multirow[t]{2}{*}{ Species } & \multirow[t]{2}{*}{ Wavelength } & \multicolumn{2}{|c|}{ NGC 330-038 } & \multicolumn{2}{|c|}{ NGC 330-039 } & \multicolumn{2}{|c|}{$\overline{\mathrm{NGC} 330-040}$} & \multicolumn{2}{|c|}{ NGC 330-041 } & \multicolumn{2}{|c|}{ NGC 330-042 } \\
\hline & & $E W$ & Abund & $E W$ & Abund & $E W$ & Abund & $E W$ & Abund & $E W$ & Abund \\
\hline C II & 4267.00 & 66 & 7.67 & - & - & 105 & 7.39 & 53 & 7.86 & 48 & 7.33 \\
\hline N II & 3955.85 & - & - & - & - & - & - & - & - & - & - \\
\hline N II & 3995.00 & $<45$ & $<7.16$ & $<35$ & $<7.61$ & - & - & - & - & 59 & 7.22 \\
\hline N II & 4447.03 & - & - & - & - & - & - & - & - & 28 & 7.21 \\
\hline N II & 4601.48 & - & - & - & - & - & - & - & - & 16 & 7.14 \\
\hline N II & 4613.86 & - & - & - & - & - & - & - & - & 11 & 7.19 \\
\hline N II & 4630.54 & - & - & - & - & $<45$ & $<7.12$ & 35 & 7.73 & 37 & 7.16 \\
\hline N II & 4643.08 & - & - & - & - & - & - & - & - & 17 & 7.16 \\
\hline O II & 3912.00 & - & - & - & - & - & - & - & - & - & - \\
\hline O II & 3919.30 & - & - & - & - & - & - & - & - & - & - \\
\hline O II & 3945.04 & 44 & 8.33 & - & - & - & - & - & - & - & - \\
\hline O II & 3954.36 & 55 & 8.15 & - & - & - & - & - & - & 25 & 7.68 \\
\hline O II & 3982.71 & - & - & - & - & - & - & - & - & - & - \\
\hline O II & 4069.00 & - & - & - & - & - & - & - & - & 65 & 7.68 \\
\hline O II & 4072.15 & - & - & - & - & - & - & - & - & 48 & 7.69 \\
\hline O II & 4075.86 & - & - & - & - & 76 & 8.12 & 77 & 8.06 & 53 & 7.59 \\
\hline O II & 4078.84 & - & - & - & - & - & - & 34 & 8.52 & - & - \\
\hline O II & 4132.80 & - & - & - & - & - & - & - & - & - & - \\
\hline O II & 4156.53 & - & - & - & - & - & - & - & - & - & - \\
\hline O II & 4185.44 & - & - & - & - & - & - & - & - & - & - \\
\hline O II & 4317.00 & 68 & 8.37 & - & - & - & - & 37 & 8.33 & 29 & 7.72 \\
\hline O II & 4319.63 & 70 & 8.40 & - & - & - & - & 35 & 8.30 & 31 & 7.77 \\
\hline O II & 4325.76 & - & - & - & - & - & - & - & - & - & - \\
\hline O II & 4349.43 & - & - & - & - & - & - & - & - & 46 & 7.77 \\
\hline O II & 4351.00 & - & - & - & - & - & - & - & - & 27 & 7.52 \\
\hline O II & 4353.58 & - & - & - & - & - & - & - & - & - & - \\
\hline O II & 4366.00 & 50 & 8.02 & - & - & - & - & - & - & 26 & 7.60 \\
\hline O II & 4369.27 & - & - & - & - & - & - & - & - & - & - \\
\hline O II & 4395.94 & - & - & - & - & - & - & - & - & - & - \\
\hline O II & 4414.90 & 116 & 8.40 & - & - & 77 & 8.20 & - & - & 39 & 7.52 \\
\hline O II & 4416.98 & 89 & 8.41 & - & - & 79 & 8.50 & - & - & 66 & 8.23 \\
\hline O II & 4443.01 & - & - & - & - & - & - & - & - & - & - \\
\hline O II & 4452.38 & - & - & - & - & - & - & - & - & - & - \\
\hline O II & 4590.97 & - & - & - & - & - & - & - & - & 35 & 7.67 \\
\hline O II & 4596.18 & - & - & - & - & - & - & - & - & 35 & 7.79 \\
\hline O II & 4638.86 & - & - & - & - & - & - & 55 & 8.46 & 39 & 7.83 \\
\hline O II & 4641.81 & - & - & - & - & - & - & 72 & 8.28 & 66 & 7.85 \\
\hline O II & 4650.00 & - & - & - & - & - & - & - & - & - & - \\
\hline O II & 4661.63 & 65 & 8.08 & - & - & - & - & - & - & 45 & 7.87 \\
\hline O II & 4673.73 & - & - & - & - & - & - & - & - & - & - \\
\hline O II & 4676.23 & - & - & - & - & - & - & - & - & 32 & 7.75 \\
\hline O II & 4699.00 & - & - & - & - & - & - & 48 & 8.17 & 26 & 7.60 \\
\hline O II & 4705.35 & - & - & - & - & - & - & - & - & 34 & 7.76 \\
\hline O II & 4710.00 & - & - & - & - & - & - & - & - & - & - \\
\hline Mg II & 4481.00 & 50 & 6.81 & - & - & 100 & 6.81 & - & - & 64 & 6.88 \\
\hline Si II & 4128.05 & - & - & - & - & - & - & - & - & - & - \\
\hline Si II & 4130.89 & - & - & - & - & - & - & - & - & - & - \\
\hline Si III & 4552.62 & 104 & 6.85 & 48 & 6.45 & 129 & 6.81 & 67 & 6.77 & 98 & 6.81 \\
\hline Si III & 4567.84 & 75 & 6.72 & 52 & 6.82 & 115 & 6.95 & - & - & 84 & 6.88 \\
\hline Si III & 4574.75 & - & - & 35 & 7.15 & - & - & - & - & 44 & 6.77 \\
\hline Si IV & 4088.90 & - & - & - & - & - & - & - & - & - & - \\
\hline Si IV & 4116.10 & - & - & - & - & - & - & - & - & 28 & 6.83 \\
\hline Si IV & 4212.00 & - & - & - & - & - & - & - & - & - & - \\
\hline
\end{tabular}


Table 1. continued.

\begin{tabular}{|c|c|c|c|c|c|c|c|c|c|c|c|}
\hline \multirow[t]{2}{*}{ Species } & \multirow[t]{2}{*}{ Wavelength } & \multicolumn{2}{|c|}{ NGC 330-045 } & \multicolumn{2}{|c|}{ NGC 330-047 } & \multicolumn{2}{|c|}{ NGC 330-048 } & \multicolumn{2}{|c|}{ NGC 330-049 } & \multicolumn{2}{|c|}{ NGC 330-051 } \\
\hline & & $E W$ & Abund & $E W$ & Abund & $E W$ & Abund & $E W$ & Abund & $E W$ & Abund \\
\hline C II & 4267.00 & 77 & 6.96 & 54 & 7.47 & 39 & 7.42 & - & - & - & - \\
\hline N II & 3955.85 & - & - & - & - & - & - & - & - & - & - \\
\hline N II & 3995.00 & $<50$ & $<7.28$ & 20 & 6.61 & $<35$ & $<7.10$ & 25 & 6.69 & $<60$ & $<7.20$ \\
\hline N II & 4447.03 & - & - & - & - & - & - & - & - & - & - \\
\hline N II & 4601.48 & - & - & - & - & - & - & - & - & - & - \\
\hline N II & 4613.86 & - & - & - & - & - & - & - & - & - & - \\
\hline N II & 4630.54 & - & - & 20 & 6.91 & - & - & - & - & - & - \\
\hline N II & 4643.08 & - & - & - & - & - & - & - & - & - & - \\
\hline O II & 3912.00 & - & - & 50 & 8.19 & - & - & - & - & - & - \\
\hline O II & 3919.30 & - & - & - & - & - & - & - & - & - & - \\
\hline O II & 3945.04 & - & - & - & - & 56 & 8.55 & - & - & - & - \\
\hline O II & 3954.36 & - & - & 45 & 8.14 & 63 & 8.30 & - & - & - & - \\
\hline O II & 3982.71 & - & - & 24 & 8.07 & - & - & - & - & - & - \\
\hline O II & 4069.00 & - & - & 88 & 7.97 & 126 & 8.14 & - & - & - & - \\
\hline O II & 4072.15 & - & - & 52 & 7.79 & 54 & 7.67 & - & - & - & - \\
\hline O II & 4075.86 & - & - & 65 & 7.84 & 75 & 7.77 & - & - & - & - \\
\hline O II & 4078.84 & - & - & 22 & 8.03 & 27 & 8.10 & - & - & - & - \\
\hline O II & 4132.80 & - & - & 34 & 8.14 & - & - & - & - & - & - \\
\hline O II & 4156.53 & - & - & - & - & - & - & - & - & - & - \\
\hline O II & 4185.44 & - & - & - & - & - & - & - & - & - & - \\
\hline O II & 4317.00 & - & - & 51 & 8.22 & 48 & 8.13 & - & - & - & - \\
\hline O II & 4319.63 & - & - & 54 & 8.28 & 53 & 8.20 & - & - & - & - \\
\hline O II & 4325.76 & - & - & 16 & 8.23 & - & - & - & - & - & - \\
\hline O II & 4349.43 & - & - & 62 & 8.14 & - & - & - & - & - & - \\
\hline O II & 4351.00 & - & - & 46 & 7.90 & - & - & - & - & - & - \\
\hline O II & 4353.58 & - & - & - & - & - & - & - & - & - & - \\
\hline O II & 4366.00 & 23 & 8.29 & 47 & 8.09 & 41 & 7.95 & - & - & - & - \\
\hline O II & 4369.27 & - & - & - & - & - & - & - & - & - & - \\
\hline O II & 4395.94 & - & - & - & - & - & - & - & - & - & - \\
\hline O II & 4414.90 & - & - & 78 & 8.13 & 84 & 8.09 & - & - & - & - \\
\hline O II & 4416.98 & - & - & 59 & 8.17 & 59 & 8.09 & - & - & - & - \\
\hline O II & 4443.01 & - & - & - & - & - & - & - & - & - & - \\
\hline O II & 4452.38 & - & - & - & - & - & - & - & - & - & - \\
\hline O II & 4590.97 & - & - & 51 & 8.01 & 68 & 8.09 & - & - & - & - \\
\hline O II & 4596.18 & - & - & 47 & 8.03 & 53 & 8.01 & - & - & - & - \\
\hline O II & 4638.86 & - & - & 49 & 8.07 & 70 & 8.27 & - & - & - & - \\
\hline O II & 4641.81 & - & - & 79 & 8.12 & 108 & 8.22 & - & - & - & - \\
\hline O II & 4650.00 & - & - & - & - & - & - & - & - & 216 & 8.33 \\
\hline O II & 4661.63 & - & - & 53 & 8.07 & 78 & 8.28 & - & - & - & - \\
\hline O II & 4673.73 & - & - & - & - & - & - & - & - & - & - \\
\hline O II & 4676.23 & - & - & 53 & 8.20 & 63 & 8.24 & - & - & - & - \\
\hline O II & 4699.00 & - & - & 46 & 7.90 & - & - & - & - & - & - \\
\hline O II & 4705.35 & - & - & 45 & 7.95 & 57 & 8.02 & - & - & - & - \\
\hline O II & 4710.00 & - & - & - & - & - & - & - & - & - & - \\
\hline Mg II & 4481.00 & - & - & 37 & 6.57 & - & - & - & - & - & - \\
\hline Si II & 4128.05 & - & - & - & - & - & - & - & - & - & - \\
\hline Si II & 4130.89 & - & - & - & - & - & - & - & - & - & - \\
\hline Si III & 4552.62 & 74 & 6.85 & 76 & 6.66 & 106 & 6.78 & - & - & 118 & 6.80 \\
\hline Si III & 4567.84 & - & - & 71 & 6.84 & 72 & 6.66 & - & - & - & - \\
\hline Si III & 4574.75 & - & - & 49 & 7.00 & 53 & 7.00 & - & - & - & - \\
\hline Si IV & 4088.90 & - & - & - & - & - & - & - & - & - & - \\
\hline Si IV & 4116.10 & - & - & 28 & 6.84 & - & - & 73 & 6.86 & - & - \\
\hline Si IV & 4212.00 & - & - & - & - & - & - & - & - & - & - \\
\hline
\end{tabular}


I. Hunter et al.: Chemical compositions of B-type stars, Online Material p 29

Table 1. continued.

\begin{tabular}{|c|c|c|c|c|c|c|c|c|c|c|c|}
\hline \multirow[t]{2}{*}{ Species } & \multirow[t]{2}{*}{ Wavelength } & \multicolumn{2}{|c|}{ NGC 330-053 } & \multicolumn{2}{|c|}{$\overline{\text { NGC 330-055 }}$} & \multicolumn{2}{|c|}{ NGC 330-056 } & \multicolumn{2}{|c|}{ NGC 330-057 } & \multicolumn{2}{|c|}{ NGC 330-059 } \\
\hline & & $E W$ & Abund & $E W$ & Abund & $E W$ & Abund & $E W$ & Abund & $E W$ & Abund \\
\hline C II & 4267.00 & 48 & 7.54 & - & - & 84 & 7.25 & - & - & 103 & 7.17 \\
\hline N II & 3955.85 & - & - & - & - & - & - & - & - & - & - \\
\hline N II & 3995.00 & $<30$ & $<7.04$ & 90 & 8.06 & $<45$ & $<6.93$ & $<65$ & $<7.48$ & 55 & 7.27 \\
\hline N II & 4447.03 & - & - & - & - & - & - & - & - & - & - \\
\hline N II & 4601.48 & - & - & - & - & - & - & - & - & - & - \\
\hline N II & 4613.86 & - & - & - & - & - & - & - & - & - & - \\
\hline N II & 4630.54 & - & - & - & - & - & - & - & - & - & - \\
\hline N II & 4643.08 & - & - & - & - & - & - & - & - & - & - \\
\hline O II & 3912.00 & - & - & - & - & - & - & - & - & - & - \\
\hline O II & 3919.30 & - & - & - & - & - & - & - & - & - & - \\
\hline O II & 3945.04 & - & - & - & - & - & - & - & - & - & - \\
\hline O II & 3954.36 & - & - & - & - & - & - & - & - & - & - \\
\hline O II & 3982.71 & - & - & - & - & - & - & - & - & - & - \\
\hline O II & 4069.00 & 135 & 8.14 & - & - & 116 & 8.59 & 90 & 7.87 & - & - \\
\hline O II & 4072.15 & 78 & 7.88 & - & - & 73 & 8.39 & 89 & 8.12 & - & - \\
\hline O II & 4075.86 & 109 & 7.99 & - & - & 87 & 8.40 & 72 & 7.73 & - & - \\
\hline O II & 4078.84 & 34 & 8.22 & - & - & - & - & - & - & - & - \\
\hline O II & 4132.80 & - & - & - & - & - & - & - & - & - & - \\
\hline O II & 4156.53 & - & - & - & - & - & - & - & - & - & - \\
\hline O II & 4185.44 & - & - & - & - & - & - & - & - & - & - \\
\hline O II & 4317.00 & 41 & 8.01 & - & - & - & - & - & - & - & - \\
\hline O II & 4319.63 & 50 & 8.12 & - & - & - & - & - & - & - & - \\
\hline O II & 4325.76 & - & - & - & - & - & - & - & - & - & - \\
\hline O II & 4349.43 & - & - & - & - & - & - & - & - & - & - \\
\hline O II & 4351.00 & - & - & - & - & - & - & - & - & - & - \\
\hline O II & 4353.58 & - & - & - & - & - & - & - & - & - & - \\
\hline O II & 4366.00 & - & - & - & - & - & - & - & - & - & - \\
\hline O II & 4369.27 & - & - & - & - & - & - & - & - & - & - \\
\hline O II & 4395.94 & - & - & - & - & - & - & - & - & - & - \\
\hline O II & 4414.90 & - & - & - & - & - & - & - & - & - & - \\
\hline O II & 4416.98 & - & - & - & - & - & - & - & - & - & - \\
\hline O II & 4443.01 & - & - & - & - & - & - & - & - & - & - \\
\hline O II & 4452.38 & - & - & - & - & - & - & - & - & - & - \\
\hline O II & 4590.97 & 52 & 7.87 & - & - & 61 & 8.54 & - & - & - & - \\
\hline O II & 4596.18 & - & - & - & - & 67 & 8.71 & - & - & - & - \\
\hline O II & 4638.86 & - & - & - & - & 53 & 8.42 & - & - & - & - \\
\hline O II & 4641.81 & - & - & - & - & 92 & 8.45 & - & - & - & - \\
\hline O II & 4650.00 & - & - & - & - & 178 & 8.49 & - & - & - & - \\
\hline O II & 4661.63 & 81 & 8.24 & - & - & 80 & 8.71 & 80 & 8.30 & - & - \\
\hline O II & 4673.73 & - & - & - & - & - & - & - & - & - & - \\
\hline O II & 4676.23 & - & - & - & - & - & - & - & - & - & - \\
\hline O II & 4699.00 & - & - & - & - & - & - & - & - & - & - \\
\hline O II & 4705.35 & 50 & 7.91 & - & - & - & - & - & - & - & - \\
\hline O II & 4710.00 & - & - & - & - & - & - & - & - & - & - \\
\hline Mg II & 4481.00 & 64 & 6.97 & - & - & 72 & 6.55 & 63 & 6.96 & - & - \\
\hline Si II & 4128.05 & - & - & - & - & - & - & - & - & - & - \\
\hline Si II & 4130.89 & - & - & - & - & - & - & - & - & - & - \\
\hline Si III & 4552.62 & 129 & 6.84 & - & - & 102 & 6.66 & - & - & 80 & 6.80 \\
\hline Si III & 4567.84 & - & - & - & - & 79 & 6.68 & - & - & - & - \\
\hline Si III & 4574.75 & - & - & - & - & 68 & 7.09 & - & - & - & - \\
\hline Si IV & 4088.90 & - & - & - & - & - & - & - & - & - & - \\
\hline Si IV & 4116.10 & - & - & - & - & - & - & - & - & - & - \\
\hline Si IV & 4212.00 & - & - & - & - & - & - & - & - & - & - \\
\hline
\end{tabular}


I. Hunter et al.: Chemical compositions of B-type stars, Online Material p 30

Table 1. continued.

\begin{tabular}{|c|c|c|c|c|c|c|c|c|c|c|c|}
\hline \multirow[t]{2}{*}{ Species } & \multirow[t]{2}{*}{ Wavelength } & \multicolumn{2}{|c|}{ NGC 330-066 } & \multicolumn{2}{|c|}{ NGC 330-067 } & \multicolumn{2}{|c|}{ NGC 330-069 } & \multicolumn{2}{|c|}{ NGC 330-072 } & \multicolumn{2}{|c|}{ NGC 330-074 } \\
\hline & & $E W$ & Abund & $E W$ & Abund & $E W$ & Abund & $E W$ & Abund & $E W$ & Abund \\
\hline C II & 4267.00 & 84 & 7.08 & 92 & 7.15 & 114 & 7.30 & 58 & 7.67 & 23 & 7.42 \\
\hline N II & 3955.85 & - & - & - & - & - & - & - & - & - & - \\
\hline N II & 3995.00 & $<70$ & $<7.77$ & 55 & 7.17 & $<90$ & $<7.91$ & $<45$ & $<7.30$ & 25 & 7.26 \\
\hline N II & 4447.03 & - & - & - & - & - & - & - & - & - & - \\
\hline N II & 4601.48 & - & - & - & - & - & - & - & - & - & - \\
\hline N II & 4613.86 & - & - & - & - & - & - & - & - & - & - \\
\hline N II & 4630.54 & - & - & 51 & 7.40 & - & - & - & - & 31 & 7.65 \\
\hline N II & 4643.08 & - & - & - & - & - & - & - & - & - & - \\
\hline O II & 3912.00 & - & - & - & - & - & - & - & - & - & - \\
\hline O II & 3919.30 & - & - & - & - & - & - & - & - & - & - \\
\hline O II & 3945.04 & - & - & - & - & - & - & - & - & - & - \\
\hline O II & 3954.36 & - & - & - & - & - & - & - & - & - & - \\
\hline O II & 3982.71 & - & - & - & - & - & - & - & - & - & - \\
\hline O II & 4069.00 & - & - & - & - & - & - & 125 & 8.15 & 77 & 8.00 \\
\hline O II & 4072.15 & - & - & - & - & - & - & 52 & 7.66 & 45 & 7.80 \\
\hline O II & 4075.86 & - & - & - & - & - & - & 67 & 7.69 & 49 & 7.69 \\
\hline O II & 4078.84 & - & - & - & - & - & - & - & - & - & - \\
\hline O II & 4132.80 & - & - & - & - & - & - & - & - & - & - \\
\hline O II & 4156.53 & - & - & - & - & - & - & - & - & - & - \\
\hline O II & 4185.44 & - & - & - & - & - & - & - & - & - & - \\
\hline O II & 4317.00 & - & - & - & - & - & - & 57 & 8.27 & - & - \\
\hline O II & 4319.63 & - & - & - & - & - & - & 49 & 8.17 & - & - \\
\hline O II & 4325.76 & - & - & - & - & - & - & - & - & - & - \\
\hline O II & 4349.43 & - & - & - & - & - & - & - & - & 46 & 8.19 \\
\hline O II & 4351.00 & - & - & - & - & - & - & - & - & 38 & 7.79 \\
\hline O II & 4353.58 & - & - & - & - & - & - & - & - & - & - \\
\hline O II & 4366.00 & - & - & - & - & - & - & - & - & - & - \\
\hline O II & 4369.27 & - & - & - & - & - & - & - & - & - & - \\
\hline O II & 4395.94 & - & - & - & - & - & - & - & - & - & - \\
\hline O II & 4414.90 & - & - & 39 & 8.00 & - & - & 81 & 8.09 & - & - \\
\hline O II & 4416.98 & - & - & 44 & 8.36 & - & - & 84 & 8.38 & - & - \\
\hline O II & 4443.01 & - & - & - & - & - & - & - & - & - & - \\
\hline O II & 4452.38 & - & - & - & - & - & - & - & - & - & - \\
\hline O II & 4590.97 & - & - & - & - & - & - & - & - & 26 & 7.78 \\
\hline O II & 4596.18 & - & - & - & - & - & - & - & - & 24 & 7.85 \\
\hline O II & 4638.86 & - & - & - & - & - & - & - & - & - & - \\
\hline O II & 4641.81 & - & - & - & - & - & - & - & - & - & - \\
\hline O II & 4650.00 & - & - & 60 & 7.86 & - & - & - & - & - & - \\
\hline O II & 4661.63 & - & - & 44 & 8.45 & - & - & 74 & 8.27 & - & - \\
\hline O II & 4673.73 & - & - & - & - & - & - & - & - & - & - \\
\hline O II & 4676.23 & - & - & - & - & - & - & - & - & - & - \\
\hline O II & 4699.00 & - & - & - & - & - & - & 71 & 8.16 & - & - \\
\hline O II & 4705.35 & - & - & - & - & - & - & - & - & - & - \\
\hline O II & 4710.00 & - & - & - & - & - & - & - & - & - & - \\
\hline Mg II & 4481.00 & - & - & - & - & 144 & 6.88 & 62 & 6.99 & 38 & 6.81 \\
\hline Si II & 4128.05 & - & - & - & - & - & - & - & - & - & - \\
\hline Si II & 4130.89 & - & - & - & - & - & - & - & - & - & - \\
\hline Si III & 4552.62 & - & - & 91 & 6.73 & - & - & 95 & 6.71 & 75 & 6.87 \\
\hline Si III & 4567.84 & - & - & 77 & 6.84 & - & - & 72 & 6.72 & 49 & 6.77 \\
\hline Si III & 4574.75 & - & - & - & - & - & - & 50 & 7.01 & 24 & 6.85 \\
\hline Si IV & 4088.90 & - & - & - & - & - & - & - & - & - & - \\
\hline Si IV & 4116.10 & - & - & - & - & - & - & - & - & 91 & 6.83 \\
\hline Si IV & 4212.00 & - & - & - & - & - & - & - & - & - & - \\
\hline
\end{tabular}


I. Hunter et al.: Chemical compositions of B-type stars, Online Material p 31

Table 1. continued.

\begin{tabular}{|c|c|c|c|c|c|c|c|c|c|c|c|}
\hline \multirow[t]{2}{*}{ Species } & \multirow[t]{2}{*}{ Wavelength } & \multicolumn{2}{|c|}{ NGC 330-079 } & \multicolumn{2}{|c|}{ NGC 330-083 } & \multicolumn{2}{|c|}{ NGC 330-086 } & \multicolumn{2}{|c|}{ NGC 330-095 } & \multicolumn{2}{|c|}{ NGC 330-097 } \\
\hline & & $E W$ & Abund & $E W$ & Abund & $E W$ & Abund & $E W$ & Abund & $E W$ & Abund \\
\hline C II & 4267.00 & 111 & 7.29 & 91 & 7.09 & 76 & 7.06 & 107 & 7.25 & 80 & 7.76 \\
\hline N II & 3955.85 & - & - & - & - & - & - & - & - & - & - \\
\hline N II & 3995.00 & $<80$ & $<7.69$ & $<60$ & $<7.66$ & $<60$ & $<7.33$ & $<25$ & $<6.95$ & 88 & 7.53 \\
\hline N II & 4447.03 & - & - & - & - & - & - & - & - & - & - \\
\hline N II & 4601.48 & - & - & - & - & - & - & - & - & - & - \\
\hline N II & 4613.86 & - & - & - & - & - & - & - & - & - & - \\
\hline $\mathrm{N}$ II & 4630.54 & - & - & - & - & - & - & - & - & 74 & 7.66 \\
\hline N II & 4643.08 & - & - & - & - & - & - & - & - & - & - \\
\hline O II & 3912.00 & - & - & - & - & - & - & - & - & - & - \\
\hline O II & 3919.30 & - & - & - & - & - & - & - & - & - & - \\
\hline O II & 3945.04 & - & - & - & - & - & - & - & - & - & - \\
\hline O II & 3954.36 & - & - & - & - & - & - & - & - & - & - \\
\hline O II & 3982.71 & - & - & - & - & - & - & - & - & - & - \\
\hline O II & 4069.00 & - & - & - & - & - & - & 40 & 8.42 & - & - \\
\hline O II & 4072.15 & - & - & - & - & - & - & - & - & - & - \\
\hline O II & 4075.86 & - & - & - & - & - & - & - & - & - & - \\
\hline O II & 4078.84 & - & - & - & - & - & - & - & - & - & - \\
\hline O II & 4132.80 & - & - & - & - & - & - & - & - & - & - \\
\hline O II & 4156.53 & - & - & - & - & - & - & - & - & - & - \\
\hline O II & 4185.44 & - & - & - & - & - & - & - & - & - & - \\
\hline O II & 4317.00 & - & - & - & - & - & - & - & - & - & - \\
\hline O II & 4319.63 & - & - & - & - & - & - & - & - & - & - \\
\hline O II & 4325.76 & - & - & - & - & - & - & - & - & - & - \\
\hline O II & 4349.43 & - & - & - & - & - & - & - & - & - & - \\
\hline O II & 4351.00 & - & - & - & - & - & - & - & - & - & - \\
\hline O II & 4353.58 & - & - & - & - & - & - & - & - & - & - \\
\hline O II & 4366.00 & - & - & - & - & - & - & - & - & 88 & 8.34 \\
\hline O II & 4369.27 & - & - & - & - & - & - & - & - & - & - \\
\hline O II & 4395.94 & - & - & - & - & - & - & - & - & - & - \\
\hline O II & 4414.90 & - & - & - & - & - & - & - & - & - & - \\
\hline O II & 4416.98 & - & - & - & - & - & - & - & - & - & - \\
\hline O II & 4443.01 & - & - & - & - & - & - & - & - & - & - \\
\hline O II & 4452.38 & - & - & - & - & - & - & - & - & - & - \\
\hline O II & 4590.97 & - & - & - & - & - & - & - & - & 99 & 8.28 \\
\hline O II & 4596.18 & - & - & - & - & - & - & - & - & 66 & 8.06 \\
\hline O II & 4638.86 & - & - & - & - & - & - & - & - & 74 & 8.15 \\
\hline O II & 4641.81 & - & - & - & - & - & - & - & - & 142 & 8.28 \\
\hline O II & 4650.00 & - & - & - & - & 51 & 7.88 & - & - & - & - \\
\hline O II & 4661.63 & - & - & - & - & - & - & - & - & 101 & 8.32 \\
\hline O II & 4673.73 & - & - & - & - & - & - & - & - & - & - \\
\hline O II & 4676.23 & - & - & - & - & - & - & - & - & - & - \\
\hline O II & 4699.00 & - & - & - & - & - & - & - & - & - & - \\
\hline O II & 4705.35 & - & - & - & - & - & - & - & - & - & - \\
\hline O II & 4710.00 & - & - & - & - & - & - & - & - & - & - \\
\hline Mg II & 4481.00 & - & - & - & - & - & - & 75 & 6.33 & - & - \\
\hline Si II & 4128.05 & - & - & - & - & - & - & - & - & - & - \\
\hline Si II & 4130.89 & - & - & - & - & - & - & - & - & - & - \\
\hline Si III & 4552.62 & - & - & - & - & 77 & 6.77 & 51 & 6.72 & 150 & 6.88 \\
\hline Si III & 4567.84 & - & - & - & - & - & - & 42 & 6.81 & 104 & 6.75 \\
\hline Si III & 4574.75 & - & - & - & - & - & - & 24 & 6.90 & - & - \\
\hline Si IV & 4088.90 & - & - & - & - & - & - & - & - & - & - \\
\hline Si IV & 4116.10 & - & - & - & - & - & - & - & - & - & - \\
\hline Si IV & 4212.00 & - & - & - & - & - & - & - & - & - & - \\
\hline
\end{tabular}


I. Hunter et al.: Chemical compositions of B-type stars, Online Material p 32

Table 1. continued.

\begin{tabular}{|c|c|c|c|c|c|c|c|c|c|c|c|}
\hline \multirow[t]{2}{*}{ Species } & \multirow[t]{2}{*}{ Wavelength } & \multicolumn{2}{|c|}{ NGC 330-101 } & \multicolumn{2}{|c|}{ NGC 330-107 } & \multicolumn{2}{|c|}{ NGC 330-108 } & \multicolumn{2}{|c|}{ NGC 330-109 } & \multicolumn{2}{|c|}{ NGC 330-110 } \\
\hline & & $E W$ & Abund & $E W$ & Abund & $E W$ & Abund & $E W$ & Abund & $E W$ & Abund \\
\hline C II & 4267.00 & 102 & 7.27 & 82 & 7.16 & - & - & - & - & 62 & 7.10 \\
\hline N II & 3955.85 & - & - & - & - & - & - & - & - & - & - \\
\hline N II & 3995.00 & 37 & 7.07 & $<30$ & $<7.42$ & - & - & - & - & 60 & 7.03 \\
\hline N II & 4447.03 & - & - & - & - & - & - & - & - & - & - \\
\hline N II & 4601.48 & - & - & - & - & - & - & - & - & - & - \\
\hline N II & 4613.86 & - & - & - & - & - & - & - & - & - & - \\
\hline N II & 4630.54 & - & - & - & - & - & - & - & - & 76 & 7.42 \\
\hline N II & 4643.08 & - & - & - & - & - & - & - & - & - & - \\
\hline O II & 3912.00 & - & - & - & - & - & - & - & - & - & - \\
\hline O II & 3919.30 & - & - & - & - & - & - & - & - & - & - \\
\hline O II & 3945.04 & - & - & - & - & - & - & - & - & - & - \\
\hline O II & 3954.36 & - & - & - & - & - & - & - & - & - & - \\
\hline O II & 3982.71 & - & - & - & - & - & - & - & - & - & - \\
\hline O II & 4069.00 & - & - & - & - & - & - & - & - & 126 & 8.49 \\
\hline O II & 4072.15 & - & - & - & - & - & - & - & - & 72 & 8.18 \\
\hline O II & 4075.86 & - & - & - & - & - & - & - & - & 97 & 8.27 \\
\hline O II & 4078.84 & - & - & - & - & - & - & - & - & - & - \\
\hline O II & 4132.80 & - & - & - & - & - & - & - & - & - & - \\
\hline O II & 4156.53 & - & - & - & - & - & - & - & - & - & - \\
\hline O II & 4185.44 & - & - & - & - & - & - & - & - & - & - \\
\hline O II & 4317.00 & 25 & 8.37 & - & - & - & - & - & - & - & - \\
\hline O II & 4319.63 & - & - & - & - & - & - & - & - & - & - \\
\hline O II & 4325.76 & - & - & - & - & - & - & - & - & - & - \\
\hline O II & 4349.43 & - & - & - & - & - & - & - & - & - & - \\
\hline O II & 4351.00 & - & - & - & - & - & - & - & - & - & - \\
\hline O II & 4353.58 & - & - & - & - & - & - & - & - & - & - \\
\hline O II & 4366.00 & 25 & 8.31 & - & - & - & - & - & - & 58 & 8.32 \\
\hline O II & 4369.27 & - & - & - & - & - & - & - & - & - & - \\
\hline O II & 4395.94 & - & - & - & - & - & - & - & - & - & - \\
\hline O II & 4414.90 & - & - & - & - & - & - & - & - & - & - \\
\hline O II & 4416.98 & - & - & - & - & - & - & - & - & - & - \\
\hline O II & 4443.01 & - & - & - & - & - & - & - & - & - & - \\
\hline O II & 4452.38 & - & - & - & - & - & - & - & - & - & - \\
\hline O II & 4590.97 & - & - & - & - & - & - & - & - & - & - \\
\hline O II & 4596.18 & - & - & - & - & - & - & - & - & 50 & 8.32 \\
\hline O II & 4638.86 & 26 & 8.39 & - & - & - & - & - & - & 47 & 8.19 \\
\hline O II & 4641.81 & 40 & 8.31 & - & - & - & - & - & - & 79 & 8.12 \\
\hline O II & 4650.00 & - & - & - & - & - & - & - & - & - & - \\
\hline O II & 4661.63 & - & - & - & - & - & - & - & - & - & - \\
\hline O II & 4673.73 & - & - & - & - & - & - & - & - & - & - \\
\hline O II & 4676.23 & - & - & - & - & - & - & - & - & 55 & 8.35 \\
\hline O II & 4699.00 & - & - & - & - & - & - & - & - & 77 & 8.64 \\
\hline O II & 4705.35 & - & - & - & - & - & - & - & - & - & - \\
\hline O II & 4710.00 & - & - & - & - & - & - & - & - & - & - \\
\hline Mg II & 4481.00 & 86 & 6.61 & 154 & 6.64 & 226 & 6.77 & 127 & 6.74 & 72 & 6.55 \\
\hline Si II & 4128.05 & - & - & - & - & - & - & - & - & - & - \\
\hline Si II & 4130.89 & - & - & - & - & - & - & - & - & - & - \\
\hline Si III & 4552.62 & 69 & 6.93 & 30 & 6.78 & - & - & - & - & 156 & 6.86 \\
\hline Si III & 4567.84 & 44 & 6.73 & - & - & - & - & - & - & 110 & 6.78 \\
\hline Si III & 4574.75 & - & - & - & - & - & - & - & - & - & - \\
\hline Si IV & 4088.90 & - & - & - & - & - & - & - & - & - & - \\
\hline Si IV & 4116.10 & - & - & - & - & - & - & - & - & - & - \\
\hline Si IV & 4212.00 & - & - & - & - & - & - & - & - & - & - \\
\hline
\end{tabular}


I. Hunter et al.: Chemical compositions of B-type stars, Online Material p 33

Table 1. continued.

\begin{tabular}{|c|c|c|c|c|c|c|c|c|c|c|c|}
\hline \multirow[t]{2}{*}{ Species } & \multirow[t]{2}{*}{ Wavelength } & \multicolumn{2}{|c|}{ NGC 330-114 } & \multicolumn{2}{|c|}{ NGC 330-116 } & \multicolumn{2}{|c|}{ NGC 330-120 } & \multicolumn{2}{|c|}{ NGC 330-123 } & \multicolumn{2}{|c|}{ NGC 330-124 } \\
\hline & & $E W$ & Abund & $E W$ & Abund & $E W$ & Abund & $E W$ & Abund & $E W$ & Abund \\
\hline C II & 4267.00 & 83 & 7.53 & 118 & 7.37 & 64 & 6.90 & - & - & 54 & 7.71 \\
\hline N II & 3955.85 & - & - & - & - & - & - & - & - & - & - \\
\hline N II & 3995.00 & 53 & 7.08 & 34 & 7.05 & $<76$ & $<7.85$ & - & - & 38 & 7.36 \\
\hline N II & 4447.03 & - & - & - & - & - & - & - & - & - & - \\
\hline N II & 4601.48 & - & - & - & - & - & - & - & - & - & - \\
\hline N II & 4613.86 & - & - & - & - & - & - & - & - & - & - \\
\hline N II & 4630.54 & 51 & 7.31 & - & - & - & - & - & - & - & - \\
\hline N II & 4643.08 & 36 & 7.57 & - & - & - & - & - & - & - & - \\
\hline O II & 3912.00 & - & - & - & - & - & - & - & - & - & - \\
\hline O II & 3919.30 & - & - & - & - & - & - & - & - & - & - \\
\hline O II & 3945.04 & - & - & - & - & - & - & - & - & - & - \\
\hline O II & 3954.36 & - & - & - & - & - & - & - & - & - & - \\
\hline O II & 3982.71 & - & - & - & - & - & - & - & - & - & - \\
\hline O II & 4069.00 & 68 & 7.92 & 56 & 8.51 & - & - & - & - & 50 & 7.62 \\
\hline O II & 4072.15 & 59 & 8.08 & - & - & - & - & - & - & - & - \\
\hline O II & 4075.86 & 56 & 7.84 & 48 & 8.50 & - & - & - & - & 55 & 7.70 \\
\hline O II & 4078.84 & - & - & - & - & - & - & - & - & - & - \\
\hline O II & 4132.80 & - & - & - & - & - & - & - & - & - & - \\
\hline O II & 4156.53 & - & - & - & - & - & - & - & - & - & - \\
\hline O II & 4185.44 & - & - & - & - & - & - & - & - & - & - \\
\hline O II & 4317.00 & 51 & 8.35 & 19 & 8.24 & - & - & - & - & - & - \\
\hline O II & 4319.63 & 29 & 7.89 & - & - & - & - & - & - & - & - \\
\hline O II & 4325.76 & - & - & - & - & - & - & - & - & - & - \\
\hline O II & 4349.43 & 62 & 8.27 & - & - & - & - & - & - & 39 & 7.94 \\
\hline O II & 4351.00 & 23 & 7.74 & - & - & - & - & - & - & - & - \\
\hline O II & 4353.58 & - & - & - & - & - & - & - & - & - & - \\
\hline O II & 4366.00 & 25 & 7.74 & - & - & - & - & - & - & - & - \\
\hline O II & 4369.27 & - & - & - & - & - & - & - & - & - & - \\
\hline O II & 4395.94 & - & - & - & - & - & - & - & - & - & - \\
\hline O II & 4414.90 & 51 & 7.86 & - & - & - & - & - & - & - & - \\
\hline O II & 4416.98 & 52 & 8.16 & - & - & - & - & - & - & - & - \\
\hline O II & 4443.01 & - & - & - & - & - & - & - & - & - & - \\
\hline O II & 4452.38 & - & - & - & - & - & - & - & - & - & - \\
\hline O II & 4590.97 & 47 & 8.14 & - & - & - & - & - & - & - & - \\
\hline O II & 4596.18 & 44 & 8.19 & - & - & - & - & - & - & - & - \\
\hline O II & 4638.86 & 39 & 8.03 & - & - & - & - & - & - & - & - \\
\hline O II & 4641.81 & 59 & 7.94 & - & - & - & - & - & - & - & - \\
\hline O II & 4650.00 & 110 & 7.92 & - & - & - & - & - & - & - & - \\
\hline O II & 4661.63 & 32 & 7.83 & - & - & - & - & - & - & - & - \\
\hline O II & 4673.73 & - & - & - & - & - & - & - & - & - & - \\
\hline O II & 4676.23 & 39 & 8.09 & - & - & - & - & - & - & - & - \\
\hline O II & 4699.00 & - & - & - & - & - & - & - & - & - & - \\
\hline O II & 4705.35 & 33 & 7.94 & - & - & - & - & - & - & - & - \\
\hline O II & 4710.00 & - & - & - & - & - & - & - & - & - & - \\
\hline Mg II & 4481.00 & 94 & 6.97 & 111 & 6.70 & - & - & 24 & 6.71 & 36 & 6.75 \\
\hline Si II & 4128.05 & - & - & - & - & - & - & - & - & - & - \\
\hline Si II & 4130.89 & - & - & - & - & - & - & - & - & - & - \\
\hline Si III & 4552.62 & 99 & 6.75 & 47 & 6.55 & - & - & - & - & 76 & 6.83 \\
\hline Si III & 4567.84 & 90 & 6.90 & 48 & 6.82 & - & - & - & - & 52 & 6.73 \\
\hline Si III & 4574.75 & 48 & 6.84 & 35 & 7.08 & - & - & - & - & 33 & 6.96 \\
\hline Si IV & 4088.90 & - & - & - & - & - & - & - & - & - & - \\
\hline Si IV & 4116.10 & - & - & - & - & - & - & 65 & 6.69 & 67 & 6.84 \\
\hline Si IV & 4212.00 & - & - & - & - & - & - & - & - & - & - \\
\hline
\end{tabular}


I. Hunter et al.: Chemical compositions of B-type stars, Online Material p 34

Table 1. continued.

\begin{tabular}{|c|c|c|c|c|c|c|c|c|c|c|c|}
\hline \multirow[t]{2}{*}{ Species } & \multirow[t]{2}{*}{ Wavelength } & \multicolumn{2}{|c|}{ NGC 330-125 } & \multicolumn{2}{|c|}{ N11-001 } & \multicolumn{2}{|c|}{ N11-002 } & \multicolumn{2}{|c|}{ N11-003 } & \multicolumn{2}{|c|}{ N11-008 } \\
\hline & & $E W$ & Abund & $E W$ & Abund & $E W$ & Abund & $E W$ & Abund & $E W$ & Abund \\
\hline C II & 4267.00 & 68 & 7.14 & 146 & 7.32 & 190 & 7.38 & 79 & 7.64 & 84 & 7.76 \\
\hline N II & 3955.85 & - & - & - & - & 42 & 8.01 & - & - & - & - \\
\hline N II & 3995.00 & 70 & 7.28 & 269 & 8.20 & 164 & 7.95 & 48 & 7.09 & 122 & 7.79 \\
\hline N II & 4447.03 & - & - & 111 & 7.96 & 66 & 7.91 & - & - & - & - \\
\hline N II & 4601.48 & - & - & 137 & 8.24 & 67 & 7.99 & - & - & - & - \\
\hline N II & 4613.86 & - & - & 96 & 8.21 & 55 & 8.09 & - & - & 35 & 7.92 \\
\hline N II & 4630.54 & 50 & 7.30 & 269 & 8.39 & 145 & 8.07 & - & - & 108 & 7.82 \\
\hline N II & 4643.08 & - & - & - & - & - & - & - & - & - & - \\
\hline O II & 3912.00 & - & - & 32 & 8.02 & - & - & 80 & 8.28 & 76 & 8.27 \\
\hline O II & 3919.30 & - & - & - & - & - & - & - & - & - & - \\
\hline O II & 3945.04 & - & - & - & - & 18 & 8.34 & 62 & 8.38 & 46 & 8.29 \\
\hline O II & 3954.36 & - & - & - & - & 18 & 8.03 & 84 & 8.21 & - & - \\
\hline O II & 3982.71 & - & - & - & - & - & - & - & - & 61 & 8.43 \\
\hline O II & 4069.00 & 83 & 8.33 & 79 & 7.97 & 48 & 8.14 & 217 & 8.46 & 199 & 8.38 \\
\hline O II & 4072.15 & 49 & 8.12 & 89 & 8.19 & 50 & 8.24 & 166 & 8.38 & 146 & 8.24 \\
\hline O II & 4075.86 & 74 & 8.35 & 97 & 8.09 & 60 & 8.21 & 193 & 8.34 & 175 & 8.20 \\
\hline O II & 4078.84 & - & - & - & - & - & - & 33 & 8.11 & 29 & 8.10 \\
\hline O II & 4132.80 & - & - & - & - & - & - & 56 & 8.35 & 61 & 8.46 \\
\hline O II & 4156.53 & - & - & - & - & - & - & - & - & - & - \\
\hline O II & 4185.44 & - & - & - & - & - & - & - & - & - & - \\
\hline O II & 4317.00 & - & - & 58 & 8.28 & 40 & 8.51 & - & - & - & - \\
\hline O II & 4319.63 & - & - & 74 & 8.45 & 50 & 8.68 & - & - & - & - \\
\hline O II & 4325.76 & - & - & - & - & 14 & 8.63 & 32 & 8.53 & - & - \\
\hline O II & 4349.43 & - & - & 113 & 8.41 & - & - & - & - & - & - \\
\hline O II & 4351.00 & - & - & - & - & - & - & - & - & - & - \\
\hline O II & 4353.58 & - & - & - & - & - & - & - & - & - & - \\
\hline O II & 4366.00 & - & - & 64 & 8.27 & 36 & 8.36 & - & - & - & - \\
\hline O II & 4369.27 & - & - & - & - & - & - & - & - & - & - \\
\hline O II & 4395.94 & - & - & - & - & - & - & - & - & - & - \\
\hline O II & 4414.90 & 73 & 8.37 & 97 & 8.19 & 53 & 8.22 & 210 & 8.49 & 153 & 8.32 \\
\hline O II & 4416.98 & 26 & 7.83 & 48 & 7.94 & 39 & 8.26 & 151 & 8.46 & 113 & 8.36 \\
\hline O II & 4443.01 & - & - & - & - & - & - & - & - & - & - \\
\hline O II & 4452.38 & - & - & - & - & - & - & - & - & 41 & 8.52 \\
\hline O II & 4590.97 & - & - & 70 & 8.24 & 32 & 8.28 & 149 & 8.36 & 117 & 8.17 \\
\hline O II & 4596.18 & - & - & - & - & 27 & 8.30 & 101 & 8.18 & 99 & 8.19 \\
\hline O II & 4638.86 & - & - & 97 & 8.55 & - & - & - & - & - & - \\
\hline O II & 4641.81 & - & - & - & - & - & - & - & - & - & - \\
\hline O II & 4650.00 & - & - & - & - & 148 & 8.47 & - & - & - & - \\
\hline O II & 4661.63 & - & - & 87 & 8.39 & - & - & 186 & 8.67 & - & - \\
\hline O II & 4673.73 & - & - & - & - & - & - & - & - & - & - \\
\hline O II & 4676.23 & 57 & 8.63 & 58 & 8.24 & 19 & 8.07 & 122 & 8.48 & - & - \\
\hline O II & 4699.00 & - & - & - & - & - & - & 65 & 8.02 & 30 & 7.72 \\
\hline O II & 4705.35 & - & - & - & - & - & - & 74 & 8.08 & 78 & 8.13 \\
\hline O II & 4710.00 & - & - & - & - & - & - & - & - & - & - \\
\hline $\mathrm{Mg}$ II & 4481.00 & 49 & 6.35 & 179 & 7.12 & 246 & 7.07 & 81 & 7.07 & 83 & 7.12 \\
\hline Si II & 4128.05 & - & - & 54 & 7.30 & 100 & 7.35 & - & - & - & - \\
\hline Si II & 4130.89 & - & - & 57 & 7.13 & 120 & 7.28 & - & - & - & - \\
\hline Si III & 4552.62 & 128 & 7.08 & 275 & 7.18 & 184 & 7.13 & 343 & 7.20 & 315 & 7.18 \\
\hline Si III & 4567.84 & 73 & 6.73 & 231 & 7.23 & 149 & 7.17 & 255 & 7.10 & 241 & 7.16 \\
\hline Si III & 4574.75 & 33 & 6.61 & 130 & 7.19 & 88 & 7.25 & 159 & 7.21 & 131 & 7.22 \\
\hline Si IV & 4088.90 & - & - & - & - & - & - & - & - & - & - \\
\hline Si IV & 4116.10 & - & - & 42 & 7.23 & - & - & 174 & 7.19 & 248 & 7.15 \\
\hline Si IV & 4212.00 & - & - & - & - & - & - & - & - & - & - \\
\hline
\end{tabular}


Table 1. continued.

\begin{tabular}{|c|c|c|c|c|c|c|c|c|c|c|c|}
\hline \multirow[t]{2}{*}{ Species } & \multirow[t]{2}{*}{ Wavelength } & \multicolumn{2}{|c|}{ N11-009 } & \multicolumn{2}{|c|}{ N11-012 } & \multicolumn{2}{|c|}{ N11-014 } & \multicolumn{2}{|c|}{ N11-015 } & \multicolumn{2}{|c|}{ N11-016 } \\
\hline & & $E W$ & Abund & $E W$ & Abund & $E W$ & Abund & $E W$ & Abund & $E W$ & Abund \\
\hline C II & 4267.00 & 187 & 7.41 & 94 & 7.35 & 188 & 7.59 & 97 & 7.75 & 135 & 7.76 \\
\hline N II & 3955.85 & - & - & - & - & - & - & - & - & - & - \\
\hline N II & 3995.00 & 89 & 7.68 & 173 & 7.72 & 202 & 7.93 & 60 & 7.14 & 217 & 7.93 \\
\hline N II & 4447.03 & 35 & 7.76 & - & - & 74 & 7.69 & - & - & - & - \\
\hline N II & 4601.48 & 28 & 7.70 & 68 & 7.70 & 92 & 7.94 & - & - & 107 & 7.99 \\
\hline N II & 4613.86 & 23 & 7.81 & 42 & 7.66 & 58 & 7.87 & - & - & 59 & 7.84 \\
\hline N II & 4630.54 & 67 & 7.77 & 157 & 7.76 & 186 & 8.02 & - & - & 177 & 7.85 \\
\hline N II & 4643.08 & - & - & - & - & - & - & - & - & - & - \\
\hline O II & 3912.00 & 13 & 8.30 & 81 & 8.39 & 49 & 8.24 & 70 & 8.18 & 61 & 8.11 \\
\hline O II & 3919.30 & - & - & - & - & - & - & - & - & - & - \\
\hline O II & 3945.04 & 11 & 8.37 & 56 & 8.36 & 33 & 8.22 & 53 & 8.27 & 61 & 8.32 \\
\hline O II & 3954.36 & 21 & 8.41 & - & - & 49 & 8.12 & 79 & 8.18 & - & - \\
\hline O II & 3982.71 & - & - & - & - & 36 & 8.26 & 57 & 8.30 & - & - \\
\hline O II & 4069.00 & 25 & 8.09 & 173 & 8.36 & 108 & 8.19 & 182 & 8.28 & 181 & 8.27 \\
\hline O II & 4072.15 & 27 & 8.22 & 167 & 8.52 & 99 & 8.29 & 156 & 8.36 & 155 & 8.33 \\
\hline O II & 4075.86 & 40 & 8.34 & 178 & 8.40 & 106 & 8.17 & 178 & 8.31 & 161 & 8.17 \\
\hline O II & 4078.84 & - & - & 25 & 8.02 & 21 & 8.08 & 34 & 8.08 & - & - \\
\hline O II & 4132.80 & - & - & 42 & 8.22 & 25 & 8.06 & 59 & 8.32 & 35 & 8.02 \\
\hline O II & 4156.53 & - & - & - & - & - & - & - & - & - & - \\
\hline O II & 4185.44 & - & - & 39 & 8.10 & - & - & - & - & 39 & 7.99 \\
\hline O II & 4317.00 & 23 & 8.48 & 130 & 8.68 & 70 & 8.41 & - & - & 110 & 8.44 \\
\hline O II & 4319.63 & 29 & 8.62 & 156 & 8.85 & 79 & 8.50 & - & - & 126 & 8.55 \\
\hline O II & 4325.76 & - & - & - & - & 19 & 8.36 & - & - & - & - \\
\hline O II & 4349.43 & 34 & 8.36 & - & - & 118 & 8.45 & - & - & 203 & 8.58 \\
\hline O II & 4351.00 & - & - & - & - & 35 & 7.86 & 94 & 8.08 & - & - \\
\hline O II & 4353.58 & - & - & - & - & - & - & - & - & - & - \\
\hline O II & 4366.00 & 25 & 8.44 & 126 & 8.56 & 69 & 8.31 & - & - & 143 & 8.56 \\
\hline O II & 4369.27 & - & - & - & - & - & - & - & - & - & - \\
\hline O II & 4395.94 & - & - & - & - & - & - & - & - & - & - \\
\hline O II & 4414.90 & 37 & 8.33 & 214 & 8.62 & 127 & 8.42 & 177 & 8.39 & 184 & 8.39 \\
\hline O II & 4416.98 & 32 & 8.49 & 154 & 8.56 & 92 & 8.39 & 142 & 8.44 & 136 & 8.37 \\
\hline O II & 4443.01 & - & - & - & - & - & - & - & - & - & - \\
\hline O II & 4452.38 & 7 & 8.41 & 47 & 8.50 & 29 & 8.39 & - & - & - & - \\
\hline O II & 4590.97 & 15 & 8.28 & 129 & 8.38 & 66 & 8.18 & 122 & 8.23 & 111 & 8.17 \\
\hline O II & 4596.18 & - & - & 110 & 8.36 & 59 & 8.21 & 128 & 8.38 & - & - \\
\hline O II & 4638.86 & 27 & 8.54 & - & - & 77 & 8.37 & 208 & 8.91 & - & - \\
\hline O II & 4641.81 & - & - & - & - & 128 & 8.34 & 310 & 8.92 & - & - \\
\hline O II & 4650.00 & 73 & 8.33 & - & - & 307 & 8.58 & - & - & - & - \\
\hline O II & 4661.63 & 28 & 8.48 & 183 & 8.73 & 94 & 8.44 & 194 & 8.74 & 167 & 8.55 \\
\hline O II & 4673.73 & - & - & - & - & - & - & - & - & - & - \\
\hline O II & 4676.23 & 23 & 8.48 & - & - & 58 & 8.22 & 151 & 8.64 & 105 & 8.32 \\
\hline O II & 4699.00 & - & - & 63 & 8.09 & - & - & 57 & 7.92 & - & - \\
\hline O II & 4705.35 & 12 & 8.27 & 79 & 8.21 & 45 & 8.09 & 97 & 8.22 & 86 & 8.16 \\
\hline O II & 4710.00 & - & - & - & - & - & - & - & - & - & - \\
\hline Mg II & 4481.00 & 261 & 6.95 & 103 & 7.02 & 175 & 7.16 & 83 & 7.01 & 144 & 7.27 \\
\hline Si II & 4128.05 & 111 & 7.24 & - & - & 44 & 7.25 & - & - & - & - \\
\hline Si II & 4130.89 & 120 & 7.11 & - & - & 43 & 7.03 & - & - & - & - \\
\hline Si III & 4552.62 & 129 & 7.19 & 336 & 7.17 & 262 & 7.19 & 304 & 7.21 & 342 & 7.21 \\
\hline Si III & 4567.84 & 91 & 7.12 & 269 & 7.14 & 213 & 7.19 & 247 & 7.20 & 281 & 7.20 \\
\hline Si III & 4574.75 & 51 & 7.19 & 159 & 7.15 & 120 & 7.14 & 146 & 7.21 & 152 & 7.10 \\
\hline Si IV & 4088.90 & - & - & - & - & - & - & - & - & - & - \\
\hline Si IV & 4116.10 & - & - & 84 & 7.14 & 25 & 6.76 & 141 & 7.23 & 94 & 7.15 \\
\hline Si IV & 4212.00 & - & - & - & - & - & - & - & - & - & - \\
\hline
\end{tabular}


Table 1. continued.

\begin{tabular}{|c|c|c|c|c|c|c|c|c|c|c|c|}
\hline \multirow[t]{2}{*}{ Species } & \multirow[t]{2}{*}{ Wavelength } & \multicolumn{2}{|c|}{ N11-017 } & \multicolumn{2}{|c|}{ N11-023 } & \multicolumn{2}{|c|}{ N11-024 } & \multicolumn{2}{|c|}{ N11-029 } & \multicolumn{2}{|c|}{ N11-034 } \\
\hline & & $E W$ & Abund & $E W$ & Abund & $E W$ & Abund & $E W$ & Abund & $E W$ & Abund \\
\hline C II & 4267.00 & 197 & 7.38 & 92 & 7.76 & 123 & 7.65 & 55 & 7.81 & 142 & 8.10 \\
\hline N II & 3955.85 & - & - & - & - & - & - & - & - & - & - \\
\hline N II & 3995.00 & 143 & 7.79 & 54 & 7.16 & 196 & 7.80 & 19 & 7.10 & 61 & 7.22 \\
\hline N II & 4447.03 & 59 & 7.79 & - & - & - & - & - & - & - & - \\
\hline N II & 4601.48 & 60 & 7.87 & - & - & 98 & 7.90 & - & - & - & - \\
\hline N II & 4613.86 & 45 & 7.92 & - & - & 66 & 7.89 & - & - & - & - \\
\hline N II & 4630.54 & 127 & 7.93 & - & - & 173 & 7.82 & - & - & 27 & 7.02 \\
\hline N II & 4643.08 & 73 & 8.01 & - & - & - & - & - & - & - & - \\
\hline O II & 3912.00 & - & - & 101 & 8.45 & 73 & 8.22 & - & - & 95 & 8.34 \\
\hline O II & 3919.30 & - & - & - & - & - & - & - & - & - & - \\
\hline O II & 3945.04 & 21 & 8.35 & 53 & 8.33 & 51 & 8.23 & 16 & 8.06 & 77 & 8.50 \\
\hline O II & 3954.36 & 26 & 8.15 & 99 & 8.36 & - & - & 27 & 7.96 & 94 & 8.29 \\
\hline O II & 3982.71 & 16 & 8.22 & - & - & - & - & - & - & - & - \\
\hline O II & 4069.00 & 62 & 8.26 & 185 & 8.34 & 170 & 8.24 & - & - & - & - \\
\hline O II & 4072.15 & 54 & 8.27 & 150 & 8.31 & 158 & 8.37 & - & - & - & - \\
\hline O II & 4075.86 & 74 & 8.35 & 194 & 8.39 & 176 & 8.30 & 102 & 8.09 & - & - \\
\hline O II & 4078.84 & 11 & 8.13 & 45 & 8.33 & 37 & 8.14 & - & - & - & - \\
\hline O II & 4132.80 & - & - & 63 & 8.44 & - & - & - & - & 67 & 8.35 \\
\hline O II & 4156.53 & - & - & - & - & - & - & - & - & - & - \\
\hline O II & 4185.44 & - & - & 60 & 8.22 & 43 & 8.05 & - & - & - & - \\
\hline O II & 4317.00 & 40 & 8.44 & - & - & 108 & 8.44 & - & - & - & - \\
\hline O II & 4319.63 & 41 & 8.46 & - & - & 131 & 8.60 & - & - & - & - \\
\hline O II & 4325.76 & - & - & - & - & 30 & 8.40 & - & - & - & - \\
\hline O II & 4349.43 & 68 & 8.46 & - & - & 185 & 8.51 & - & - & - & - \\
\hline O II & 4351.00 & 16 & 7.95 & - & - & 61 & 7.88 & 45 & 7.87 & - & - \\
\hline O II & 4353.58 & - & - & - & - & - & - & - & - & - & - \\
\hline O II & 4366.00 & 40 & 8.35 & - & - & 111 & 8.36 & - & - & - & - \\
\hline O II & 4369.27 & - & - & - & - & - & - & - & - & - & - \\
\hline O II & 4395.94 & - & - & - & - & - & - & - & - & 60 & 8.54 \\
\hline O II & 4414.90 & 61 & 8.28 & 189 & 8.47 & 184 & 8.41 & 79 & 8.37 & - & - \\
\hline O II & 4416.98 & 40 & 8.23 & 144 & 8.49 & 126 & 8.33 & 56 & 8.39 & - & - \\
\hline O II & 4443.01 & - & - & - & - & - & - & - & - & - & - \\
\hline O II & 4452.38 & 17 & 8.48 & - & - & 50 & 8.46 & - & - & - & - \\
\hline O II & 4590.97 & 34 & 8.30 & 156 & 8.44 & 118 & 8.25 & 57 & 8.11 & 151 & 8.38 \\
\hline O II & 4596.18 & 32 & 8.38 & 136 & 8.44 & 100 & 8.23 & 64 & 8.30 & 127 & 8.34 \\
\hline O II & 4638.86 & 46 & 8.47 & 152 & 8.65 & 132 & 8.46 & 63 & 8.58 & - & - \\
\hline O II & 4641.81 & 79 & 8.43 & 275 & 8.76 & - & - & 154 & 8.73 & - & - \\
\hline O II & 4650.00 & 148 & 8.43 & - & - & - & - & - & - & - & - \\
\hline O II & 4661.63 & 52 & 8.48 & 173 & 8.67 & 151 & 8.49 & 73 & 8.58 & 198 & 8.75 \\
\hline O II & 4673.73 & - & - & - & - & - & - & - & - & - & - \\
\hline O II & 4676.23 & 38 & 8.40 & - & - & 106 & 8.34 & 66 & 8.64 & - & - \\
\hline O II & 4699.00 & - & - & 88 & 8.20 & - & - & - & - & 107 & 8.25 \\
\hline O II & 4705.35 & 31 & 8.41 & 84 & 8.16 & 95 & 8.24 & 30 & 7.94 & 89 & 8.14 \\
\hline O II & 4710.00 & - & - & - & - & - & - & - & - & - & - \\
\hline $\mathrm{Mg}$ II & 4481.00 & 218 & 7.00 & 74 & 7.00 & 127 & 7.14 & 49 & 6.93 & 129 & 7.33 \\
\hline Si II & 4128.05 & 78 & 7.20 & - & - & - & - & - & - & - & - \\
\hline Si II & 4130.89 & 88 & 7.08 & - & - & - & - & - & - & - & - \\
\hline Si III & 4552.62 & 189 & 7.19 & 317 & 7.20 & 322 & 7.15 & 159 & 7.15 & 340 & 7.44 \\
\hline Si III & 4567.84 & 152 & 7.22 & 245 & 7.16 & 261 & 7.13 & 121 & 7.18 & 301 & 7.55 \\
\hline Si III & 4574.75 & 79 & 7.16 & 133 & 7.15 & 159 & 7.16 & 55 & 7.19 & 184 & 7.54 \\
\hline Si IV & 4088.90 & - & - & - & - & - & - & - & - & - & - \\
\hline Si IV & 4116.10 & - & - & 182 & 7.18 & 83 & 7.15 & 248 & 6.93 & - & - \\
\hline Si IV & 4212.00 & - & - & - & - & - & - & - & - & - & - \\
\hline
\end{tabular}


Table 1. continued.

\begin{tabular}{|c|c|c|c|c|c|c|c|c|c|c|c|}
\hline \multirow[t]{2}{*}{ Species } & \multirow[t]{2}{*}{ Wavelength } & \multicolumn{2}{|c|}{ N11-036 } & \multicolumn{2}{|c|}{ N11-037 } & \multicolumn{2}{|c|}{ N11-039 } & \multicolumn{2}{|c|}{ N11-042 } & \multicolumn{2}{|c|}{ N11-046 } \\
\hline & & $E W$ & Abund & $E W$ & Abund & $E W$ & Abund & $E W$ & Abund & $E W$ & Abund \\
\hline C II & 4267.00 & 91 & 7.66 & 65 & 7.81 & 202 & 8.02 & 61 & 7.75 & 37 & 7.90 \\
\hline N II & 3955.85 & - & - & - & - & - & - & - & - & - & - \\
\hline N II & 3995.00 & 154 & 7.74 & $<27$ & $<7.17$ & 53 & 6.89 & 18 & 6.92 & $<31$ & $<7.71$ \\
\hline N II & 4447.03 & 80 & 7.74 & - & - & - & - & - & - & - & - \\
\hline N II & 4601.48 & - & - & - & - & - & - & - & - & - & - \\
\hline N II & 4613.86 & 46 & 7.82 & - & - & - & - & - & - & - & - \\
\hline N II & 4630.54 & 131 & 7.75 & - & - & 37 & 6.94 & - & - & - & - \\
\hline N II & 4643.08 & - & - & - & - & - & - & - & - & - & - \\
\hline O II & 3912.00 & 72 & 8.17 & 47 & 8.19 & 59 & 8.17 & 50 & 8.21 & - & - \\
\hline O II & 3919.30 & - & - & - & - & - & - & - & - & - & - \\
\hline O II & 3945.04 & 59 & 8.32 & 31 & 8.29 & 46 & 8.25 & 28 & 8.22 & - & - \\
\hline O II & 3954.36 & 95 & 8.29 & 49 & 8.19 & 53 & 8.02 & 50 & 8.21 & - & - \\
\hline O II & 3982.71 & 58 & 8.30 & - & - & - & - & 31 & 8.27 & - & - \\
\hline O II & 4069.00 & 166 & 8.17 & - & - & - & - & 156 & 8.54 & - & - \\
\hline O II & 4072.15 & 150 & 8.29 & - & - & - & - & 87 & 8.25 & - & - \\
\hline O II & 4075.86 & 176 & 8.27 & 116 & 8.16 & - & - & 104 & 8.27 & - & - \\
\hline O II & 4078.84 & 36 & 8.10 & - & - & - & - & 22 & 8.13 & - & - \\
\hline O II & 4132.80 & 45 & 8.11 & - & - & - & - & 43 & 8.37 & 36 & 8.55 \\
\hline O II & 4156.53 & - & - & - & - & - & - & - & - & - & - \\
\hline O II & 4185.44 & 65 & 8.22 & - & - & - & - & 30 & 7.93 & - & - \\
\hline O II & 4317.00 & 109 & 8.46 & - & - & - & - & - & - & - & - \\
\hline O II & 4319.63 & 128 & 8.59 & - & - & - & - & - & - & - & - \\
\hline O II & 4325.76 & 35 & 8.49 & - & - & - & - & 28 & 8.84 & - & - \\
\hline O II & 4349.43 & 204 & 8.65 & - & - & - & - & - & - & - & - \\
\hline O II & 4351.00 & 107 & 8.18 & - & - & - & - & 56 & 7.94 & - & - \\
\hline O II & 4353.58 & - & - & - & - & - & - & - & - & - & - \\
\hline O II & 4366.00 & 110 & 8.38 & - & - & 55 & 7.97 & - & - & - & - \\
\hline O II & 4369.27 & - & - & - & - & - & - & - & - & - & - \\
\hline O II & 4395.94 & - & - & - & - & - & - & - & - & - & - \\
\hline O II & 4414.90 & 186 & 8.42 & - & - & - & - & 89 & 8.31 & - & - \\
\hline O II & 4416.98 & 146 & 8.45 & - & - & - & - & 72 & 8.40 & - & - \\
\hline O II & 4443.01 & - & - & - & - & - & - & - & - & - & - \\
\hline O II & 4452.38 & 50 & 8.46 & - & - & - & - & - & - & - & - \\
\hline O II & 4590.97 & 124 & 8.25 & - & - & 67 & 8.00 & 71 & 8.20 & 33 & 8.30 \\
\hline O II & 4596.18 & 111 & 8.27 & 57 & 8.14 & 53 & 7.96 & - & - & 37 & 8.45 \\
\hline O II & 4638.86 & 122 & 8.41 & - & - & - & - & 78 & 8.59 & - & - \\
\hline O II & 4641.81 & - & - & - & - & - & - & 130 & 8.65 & - & - \\
\hline O II & 4650.00 & - & - & - & - & 329 & 8.49 & 206 & 8.49 & - & - \\
\hline O II & 4661.63 & 159 & 8.54 & - & - & 101 & 8.32 & 82 & 8.55 & - & - \\
\hline O II & 4673.73 & - & - & - & - & - & - & - & - & - & - \\
\hline O II & 4676.23 & 105 & 8.35 & - & - & - & - & 62 & 8.48 & - & - \\
\hline O II & 4699.00 & 85 & 8.13 & 46 & 8.10 & 44 & 7.87 & - & - & - & - \\
\hline O II & 4705.35 & 102 & 8.25 & 58 & 8.17 & 43 & 7.85 & 66 & 8.25 & - & - \\
\hline O II & 4710.00 & - & - & - & - & - & - & - & - & - & - \\
\hline Mg II & 4481.00 & 95 & 7.03 & 59 & 7.01 & 186 & 7.40 & 59 & 7.00 & 52 & 7.10 \\
\hline Si II & 4128.05 & - & - & - & - & - & - & - & - & - & - \\
\hline Si II & 4130.89 & - & - & - & - & - & - & - & - & - & - \\
\hline Si III & 4552.62 & 294 & 7.17 & 174 & 7.11 & 265 & 7.26 & 137 & 7.24 & 49 & 7.08 \\
\hline Si III & 4567.84 & 240 & 7.17 & 144 & 7.21 & 211 & 7.20 & 110 & 7.25 & - & - \\
\hline Si III & 4574.75 & 140 & 7.17 & 68 & 7.21 & 123 & 7.14 & 52 & 7.14 & - & - \\
\hline Si IV & 4088.90 & - & - & - & - & - & - & - & - & - & - \\
\hline Si IV & 4116.10 & 114 & 7.16 & 227 & 7.03 & - & - & 144 & 7.32 & - & - \\
\hline Si IV & 4212.00 & - & - & - & - & - & - & - & - & - & - \\
\hline
\end{tabular}


Table 1. continued.

\begin{tabular}{|c|c|c|c|c|c|c|c|c|c|c|c|}
\hline \multirow[t]{2}{*}{ Species } & \multirow[t]{2}{*}{ Wavelength } & \multicolumn{2}{|c|}{ N11-047 } & \multicolumn{2}{|c|}{ N11-054 } & \multicolumn{2}{|c|}{ N11-059 } & \multicolumn{2}{|c|}{ N11-062 } & \multicolumn{2}{|c|}{ N11-069 } \\
\hline & & $E W$ & Abund & $E W$ & Abund & $E W$ & Abund & $E W$ & Abund & $E W$ & Abund \\
\hline C II & 4267.00 & 77 & 7.89 & 115 & 7.83 & - & - & 48 & 7.61 & 131 & 7.92 \\
\hline N II & 3955.85 & - & - & - & - & - & - & - & - & - & - \\
\hline N II & 3995.00 & $<16$ & $<6.88$ & 33 & 6.79 & - & - & 30 & 7.20 & 46 & 6.94 \\
\hline N II & 4447.03 & - & - & - & - & - & - & 14 & 7.12 & - & - \\
\hline N II & 4601.48 & - & - & - & - & - & - & - & - & - & - \\
\hline N II & 4613.86 & - & - & - & - & - & - & - & - & - & - \\
\hline N II & 4630.54 & - & - & 28 & 6.93 & - & - & - & - & - & - \\
\hline N II & 4643.08 & - & - & - & - & - & - & - & - & - & - \\
\hline O II & 3912.00 & 58 & 8.27 & 104 & 8.44 & - & - & 53 & 8.25 & 131 & 8.59 \\
\hline O II & 3919.30 & - & - & - & - & - & - & - & - & - & - \\
\hline O II & 3945.04 & 28 & 8.19 & 66 & 8.39 & 16 & 8.54 & 29 & 8.25 & 69 & 8.42 \\
\hline O II & 3954.36 & 46 & 8.13 & 96 & 8.30 & 19 & 8.34 & 50 & 8.26 & 101 & 8.34 \\
\hline O II & 3982.71 & - & - & 68 & 8.40 & - & - & - & - & 75 & 8.47 \\
\hline O II & 4069.00 & - & - & 188 & 8.31 & - & - & 117 & 8.21 & 234 & 8.53 \\
\hline O II & 4072.15 & - & - & 156 & 8.38 & 65 & 8.46 & 73 & 8.06 & 169 & 8.42 \\
\hline O II & 4075.86 & 122 & 8.24 & 211 & 8.55 & 57 & 8.20 & 84 & 8.01 & 190 & 8.36 \\
\hline O II & 4078.84 & - & - & 54 & 8.39 & - & - & 24 & 8.17 & 56 & 8.38 \\
\hline O II & 4132.80 & 41 & 8.28 & 63 & 8.33 & - & - & 40 & 8.27 & 64 & 8.30 \\
\hline O II & 4156.53 & - & - & - & - & 23 & 9.20 & - & - & - & - \\
\hline O II & 4185.44 & - & - & 51 & 8.09 & - & - & 33 & 7.96 & 62 & 8.18 \\
\hline O II & 4317.00 & - & - & 131 & 8.64 & - & - & 51 & 8.40 & 163 & 8.84 \\
\hline O II & 4319.63 & - & - & 146 & 8.75 & - & - & 66 & 8.59 & 146 & 8.71 \\
\hline O II & 4325.76 & 23 & 8.69 & - & - & - & - & 23 & 8.68 & - & - \\
\hline O II & 4349.43 & - & - & 185 & 8.56 & - & - & - & - & 143 & 8.29 \\
\hline O II & 4351.00 & 37 & 7.67 & 101 & 8.15 & - & - & 53 & 7.93 & 68 & 7.88 \\
\hline O II & 4353.58 & - & - & - & - & - & - & - & - & - & - \\
\hline O II & 4366.00 & - & - & 133 & 8.55 & - & - & 55 & 8.38 & 130 & 8.50 \\
\hline O II & 4369.27 & - & - & - & - & - & - & - & - & - & - \\
\hline O II & 4395.94 & - & - & - & - & - & - & - & - & - & - \\
\hline O II & 4414.90 & 73 & 8.10 & 206 & 8.55 & - & - & 82 & 8.25 & 217 & 8.58 \\
\hline O II & 4416.98 & 55 & 8.16 & 148 & 8.48 & - & - & 69 & 8.37 & 162 & 8.55 \\
\hline O II & 4443.01 & - & - & - & - & - & - & - & - & - & - \\
\hline O II & 4452.38 & - & - & 52 & 8.48 & - & - & 25 & 8.44 & 69 & 8.65 \\
\hline O II & 4590.97 & 85 & 8.26 & 150 & 8.44 & - & - & 54 & 8.03 & 147 & 8.41 \\
\hline O II & 4596.18 & 60 & 8.13 & 135 & 8.44 & - & - & 52 & 8.11 & 132 & 8.42 \\
\hline O II & 4638.86 & 65 & 8.41 & 171 & 8.70 & - & - & 59 & 8.34 & 172 & 8.69 \\
\hline O II & 4641.81 & 128 & 8.49 & 260 & 8.71 & - & - & 96 & 8.32 & 243 & 8.60 \\
\hline O II & 4650.00 & - & - & - & - & - & - & 180 & 8.33 & - & - \\
\hline O II & 4661.63 & 74 & 8.42 & 168 & 8.60 & - & - & 69 & 8.39 & 190 & 8.71 \\
\hline O II & 4673.73 & - & - & - & - & - & - & - & - & - & - \\
\hline O II & 4676.23 & 70 & 8.49 & 133 & 8.52 & - & - & 56 & 8.36 & 105 & 8.33 \\
\hline O II & 4699.00 & 47 & 8.02 & 96 & 8.20 & - & - & 44 & 7.98 & 93 & 8.18 \\
\hline O II & 4705.35 & 67 & 8.20 & 112 & 8.33 & 34 & 8.55 & 52 & 8.07 & 92 & 8.17 \\
\hline O II & 4710.00 & - & - & - & - & - & - & - & - & - & - \\
\hline Mg II & 4481.00 & 61 & 7.00 & 88 & 6.98 & 61 & 7.20 & 57 & 6.99 & 102 & 7.06 \\
\hline Si II & 4128.05 & - & - & - & - & - & - & - & - & - & - \\
\hline Si II & 4130.89 & - & - & - & - & - & - & - & - & - & - \\
\hline Si III & 4552.62 & 159 & 7.18 & 289 & 7.21 & 49 & 7.35 & 117 & 7.14 & 277 & 7.13 \\
\hline Si III & 4567.84 & 130 & 7.24 & 227 & 7.14 & 49 & 7.59 & 98 & 7.21 & 236 & 7.20 \\
\hline Si III & 4574.75 & 63 & 7.19 & 132 & 7.13 & - & - & 48 & 7.14 & 139 & 7.20 \\
\hline Si IV & 4088.90 & - & - & - & - & - & - & - & - & - & - \\
\hline Si IV & 4116.10 & 169 & 7.19 & 105 & 7.14 & - & - & 115 & 7.18 & 105 & 7.18 \\
\hline Si IV & 4212.00 & - & - & - & - & - & - & - & - & - & - \\
\hline
\end{tabular}


Table 1. continued.

\begin{tabular}{|c|c|c|c|c|c|c|c|c|c|c|c|}
\hline \multirow[t]{2}{*}{ Species } & \multirow[t]{2}{*}{ Wavelength } & \multicolumn{2}{|c|}{ N11-070 } & \multicolumn{2}{|c|}{ N11-072 } & \multicolumn{2}{|c|}{ N11-075 } & \multicolumn{2}{|c|}{ N11-077 } & \multicolumn{2}{|c|}{ N11-079 } \\
\hline & & $E W$ & Abund & $E W$ & Abund & $E W$ & Abund & $E W$ & Abund & $E W$ & Abund \\
\hline C II & 4267.00 & 132 & 7.48 & 74 & 7.82 & 147 & 7.74 & 139 & 7.66 & 43 & 7.74 \\
\hline N II & 3955.85 & - & - & - & - & - & - & - & - & - & - \\
\hline N II & 3995.00 & 24 & 6.75 & 52 & 7.41 & 133 & 7.84 & 46 & 6.92 & 13 & 7.01 \\
\hline N II & 4447.03 & - & - & 25 & 7.35 & 76 & 7.82 & - & - & - & - \\
\hline N II & 4601.48 & - & - & - & - & 85 & 8.16 & - & - & - & - \\
\hline N II & 4613.86 & - & - & - & - & - & - & - & - & - & - \\
\hline N II & 4630.54 & 20 & 6.95 & 34 & 7.38 & 127 & 8.05 & 36 & 7.00 & 12 & 7.20 \\
\hline N II & 4643.08 & - & - & - & - & 84 & 8.12 & - & - & - & - \\
\hline O II & 3912.00 & 26 & 8.37 & 60 & 8.26 & 41 & 8.14 & 56 & 8.45 & 29 & 8.11 \\
\hline O II & 3919.30 & - & - & - & - & - & - & - & - & - & - \\
\hline O II & 3945.04 & - & - & 38 & 8.33 & 29 & 8.18 & 41 & 8.51 & 15 & 8.11 \\
\hline O II & 3954.36 & - & - & 61 & 8.30 & 40 & 8.09 & 50 & 8.34 & 22 & 8.00 \\
\hline O II & 3982.71 & - & - & 35 & 8.28 & 24 & 8.10 & - & - & 14 & 8.09 \\
\hline O II & 4069.00 & 43 & 8.15 & - & - & 91 & 8.12 & 141 & 8.74 & 117 & 8.52 \\
\hline O II & 4072.15 & 32 & 8.21 & 92 & 8.23 & 60 & 8.03 & 67 & 8.28 & 47 & 7.99 \\
\hline O II & 4075.86 & 46 & 8.43 & 101 & 8.13 & 66 & 7.96 & 102 & 8.65 & 48 & 7.82 \\
\hline O II & 4078.84 & - & - & 30 & 8.24 & 20 & 8.08 & 26 & 8.32 & - & - \\
\hline O II & 4132.80 & - & - & 43 & 8.28 & 29 & 8.13 & - & - & 23 & 8.13 \\
\hline O II & 4156.53 & - & - & - & - & - & - & - & - & - & - \\
\hline O II & 4185.44 & - & - & 50 & 8.17 & - & - & - & - & - & - \\
\hline O II & 4317.00 & 23 & 8.22 & 59 & 8.42 & 52 & 8.28 & 70 & 8.69 & 29 & 8.33 \\
\hline O II & 4319.63 & 24 & 8.26 & 72 & 8.58 & 52 & 8.28 & 60 & 8.52 & 32 & 8.38 \\
\hline O II & 4325.76 & - & - & 40 & 8.94 & 21 & 8.46 & - & - & - & - \\
\hline O II & 4349.43 & - & - & 109 & 8.63 & 78 & 8.33 & - & - & 51 & 8.46 \\
\hline O II & 4351.00 & - & - & 64 & 7.98 & 53 & 8.22 & - & - & 35 & 7.84 \\
\hline O II & 4353.58 & - & - & - & - & - & - & - & - & - & - \\
\hline O II & 4366.00 & 23 & 8.18 & 72 & 8.50 & 52 & 8.21 & 52 & 8.31 & 32 & 8.34 \\
\hline O II & 4369.27 & - & - & - & - & - & - & - & - & - & - \\
\hline O II & 4395.94 & - & - & - & - & - & - & - & - & - & - \\
\hline O II & 4414.90 & 30 & 8.01 & 105 & 8.33 & 74 & 8.13 & 79 & 8.31 & 36 & 8.03 \\
\hline O II & 4416.98 & 26 & 8.19 & 88 & 8.45 & 69 & 8.35 & 66 & 8.43 & 28 & 8.12 \\
\hline O II & 4443.01 & - & - & - & - & - & - & - & - & - & - \\
\hline O II & 4452.38 & - & - & 32 & 8.48 & - & - & - & - & - & - \\
\hline O II & 4590.97 & 23 & 8.22 & 77 & 8.19 & 47 & 8.07 & 65 & 8.46 & 31 & 7.95 \\
\hline O II & 4596.18 & - & - & - & - & 47 & 8.17 & 60 & 8.45 & 23 & 7.89 \\
\hline O II & 4638.86 & 23 & 8.22 & 78 & 8.46 & 58 & 8.30 & 61 & 8.44 & 34 & 8.29 \\
\hline O II & 4641.81 & 40 & 8.29 & 123 & 8.46 & 85 & 8.20 & 105 & 8.59 & 58 & 8.29 \\
\hline O II & 4650.00 & 82 & 8.35 & 220 & 8.42 & 152 & 8.15 & 188 & 8.52 & 196 & 8.86 \\
\hline O II & 4661.63 & 24 & 8.19 & 81 & 8.42 & 60 & 8.24 & 72 & 8.54 & 34 & 8.23 \\
\hline O II & 4673.73 & - & - & - & - & - & - & - & - & - & - \\
\hline O II & 4676.23 & - & - & 62 & 8.35 & 43 & 8.11 & - & - & 26 & 8.19 \\
\hline O II & 4699.00 & - & - & - & - & - & - & 48 & 8.19 & 29 & 7.96 \\
\hline O II & 4705.35 & - & - & 72 & 8.24 & 45 & 8.16 & 33 & 8.00 & 29 & 7.92 \\
\hline O II & 4710.00 & - & - & - & - & - & - & - & - & 12 & 8.31 \\
\hline Mg II & 4481.00 & 105 & 6.86 & 75 & 7.12 & 139 & 7.16 & 104 & 6.94 & 45 & 6.96 \\
\hline Si II & 4128.05 & - & - & - & - & 34 & 7.37 & - & - & - & - \\
\hline Si II & 4130.89 & - & - & - & - & 32 & 7.10 & - & - & - & - \\
\hline Si III & 4552.62 & 77 & 7.23 & 141 & 7.20 & 153 & 7.16 & 136 & 7.20 & 55 & 6.91 \\
\hline Si III & 4567.84 & 62 & 7.22 & 114 & 7.21 & 121 & 7.11 & 110 & 7.17 & 48 & 7.04 \\
\hline Si III & 4574.75 & 40 & 7.27 & 64 & 7.21 & 86 & 7.25 & 75 & 7.26 & 22 & 7.02 \\
\hline Si IV & 4088.90 & - & - & - & - & - & - & - & - & - & - \\
\hline Si IV & 4116.10 & - & - & 125 & 7.30 & 26 & 7.23 & - & - & 60 & 6.51 \\
\hline Si IV & 4212.00 & - & - & 26 & 7.17 & - & - & - & - & - & - \\
\hline
\end{tabular}


Table 1. continued.

\begin{tabular}{|c|c|c|c|c|c|c|c|c|c|c|c|}
\hline \multirow[t]{2}{*}{ Species } & \multirow[t]{2}{*}{ Wavelength } & \multicolumn{2}{|c|}{ N11-083 } & \multicolumn{2}{|c|}{ N11-084 } & \multicolumn{2}{|c|}{ N11-086 } & \multicolumn{2}{|c|}{ N11-088 } & \multicolumn{2}{|c|}{ N11-089 } \\
\hline & & $E W$ & Abund & $E W$ & Abund & $E W$ & Abund & $E W$ & Abund & $E W$ & Abund \\
\hline C II & 4267.00 & 76 & 7.85 & 69 & 7.76 & 48 & 7.36 & 84 & 7.59 & 168 & 7.88 \\
\hline N II & 3955.85 & - & - & - & - & - & - & - & - & - & - \\
\hline N II & 3995.00 & 19 & 6.86 & $<21$ & $<6.89$ & 28 & 6.84 & $<49$ & $<6.96$ & 47 & 6.88 \\
\hline N II & 4447.03 & - & - & - & - & - & - & - & - & - & - \\
\hline N II & 4601.48 & - & - & - & - & - & - & - & - & - & - \\
\hline N II & 4613.86 & - & - & - & - & - & - & - & - & - & - \\
\hline N II & 4630.54 & - & - & - & - & 14 & 6.81 & - & - & 35 & 6.95 \\
\hline $\mathrm{N}$ II & 4643.08 & - & - & - & - & - & - & - & - & - & - \\
\hline O II & 3912.00 & 40 & 8.06 & 60 & 8.34 & 56 & 8.30 & 69 & 8.22 & 37 & 8.02 \\
\hline O II & 3919.30 & - & - & - & - & - & - & - & - & - & - \\
\hline O II & 3945.04 & 25 & 8.14 & 48 & 8.58 & 28 & 8.15 & - & - & - & - \\
\hline O II & 3954.36 & 45 & 8.19 & 52 & 8.30 & 22 & 7.70 & 66 & 8.14 & 41 & 8.03 \\
\hline O II & 3982.71 & 32 & 8.31 & - & - & - & - & - & - & - & - \\
\hline O II & 4069.00 & 133 & 8.43 & - & - & 75 & 7.87 & - & - & - & - \\
\hline O II & 4072.15 & 72 & 8.16 & - & - & 50 & 7.80 & - & - & - & - \\
\hline O II & 4075.86 & 81 & 8.11 & - & - & 59 & 7.78 & - & - & 78 & 8.04 \\
\hline O II & 4078.84 & 36 & 8.46 & - & - & - & - & - & - & - & - \\
\hline O II & 4132.80 & 36 & 8.20 & - & - & - & - & 63 & 8.37 & - & - \\
\hline O II & 4156.53 & - & - & - & - & - & - & - & - & - & - \\
\hline O II & 4185.44 & 47 & 8.21 & - & - & - & - & - & - & - & - \\
\hline O II & 4317.00 & - & - & - & - & 49 & 8.20 & 83 & 8.34 & 57 & 8.29 \\
\hline O II & 4319.63 & 31 & 7.96 & - & - & 45 & 8.12 & 94 & 8.44 & 54 & 8.24 \\
\hline O II & 4325.76 & 26 & 8.66 & - & - & - & - & - & - & - & - \\
\hline O II & 4349.43 & 92 & 8.73 & - & - & 53 & 8.04 & - & - & - & - \\
\hline O II & 4351.00 & 73 & 8.24 & - & - & - & - & - & - & - & - \\
\hline O II & 4353.58 & - & - & - & - & - & - & - & - & - & - \\
\hline O II & 4366.00 & 66 & 8.54 & - & - & - & - & - & - & 38 & 7.89 \\
\hline O II & 4369.27 & 18 & 8.24 & - & - & - & - & - & - & - & - \\
\hline O II & 4395.94 & - & - & - & - & - & - & - & - & - & - \\
\hline O II & 4414.90 & 94 & 8.38 & - & - & 76 & 8.13 & - & - & - & - \\
\hline O II & 4416.98 & 80 & 8.51 & - & - & 56 & 8.14 & - & - & - & - \\
\hline O II & 4443.01 & - & - & - & - & - & - & - & - & - & - \\
\hline O II & 4452.38 & 32 & 8.50 & - & - & - & - & - & - & - & - \\
\hline O II & 4590.97 & 69 & 8.28 & 61 & 8.16 & 52 & 8.05 & 130 & 8.50 & 36 & 7.79 \\
\hline O II & 4596.18 & 72 & 8.40 & 52 & 8.11 & 47 & 8.06 & 92 & 8.27 & 38 & 7.93 \\
\hline O II & 4638.86 & 63 & 8.38 & 66 & 8.41 & 47 & 8.08 & 127 & 8.58 & 58 & 8.20 \\
\hline O II & 4641.81 & 94 & 8.38 & 98 & 8.42 & 62 & 7.89 & 188 & 8.54 & 81 & 8.06 \\
\hline O II & 4650.00 & 176 & 8.36 & - & - & - & - & - & - & 176 & 8.21 \\
\hline O II & 4661.63 & 69 & 8.39 & 56 & 8.19 & 48 & 8.03 & 125 & 8.48 & 46 & 7.95 \\
\hline O II & 4673.73 & - & - & - & - & - & - & - & - & - & - \\
\hline O II & 4676.23 & 62 & 8.41 & - & - & - & - & - & - & - & - \\
\hline O II & 4699.00 & - & - & 60 & 8.07 & 46 & 7.91 & - & - & - & - \\
\hline O II & 4705.35 & 61 & 8.19 & 37 & 7.81 & - & - & - & - & 38 & 7.95 \\
\hline O II & 4710.00 & - & - & - & - & - & - & - & - & - & - \\
\hline Mg II & 4481.00 & 57 & 7.00 & 53 & 6.94 & 53 & 6.79 & 87 & 6.93 & 157 & 7.30 \\
\hline Si II & 4128.05 & - & - & - & - & - & - & - & - & - & - \\
\hline Si II & 4130.89 & - & - & - & - & - & - & - & - & - & - \\
\hline Si III & 4552.62 & 86 & 7.00 & 82 & 6.95 & 74 & 6.66 & 200 & 6.99 & 178 & 7.30 \\
\hline Si III & 4567.84 & 73 & 7.06 & 59 & 6.82 & 58 & 6.65 & 206 & 7.32 & 136 & 7.17 \\
\hline Si III & 4574.75 & 44 & 7.11 & 37 & 6.97 & 41 & 6.86 & 133 & 7.34 & 82 & 7.12 \\
\hline Si IV & 4088.90 & - & - & - & - & - & - & - & - & - & - \\
\hline Si IV & 4116.10 & 61 & 7.06 & - & - & - & - & - & - & - & - \\
\hline Si IV & 4212.00 & - & - & - & - & - & - & - & - & - & - \\
\hline
\end{tabular}


I. Hunter et al.: Chemical compositions of B-type stars, Online Material p 41

Table 1. continued.

\begin{tabular}{|c|c|c|c|c|c|c|c|c|c|c|c|}
\hline \multirow[t]{2}{*}{ Species } & \multirow[t]{2}{*}{ Wavelength } & \multicolumn{2}{|c|}{ N11-093 } & \multicolumn{2}{|c|}{ N11-095 } & \multicolumn{2}{|c|}{ N11-097 } & \multicolumn{2}{|c|}{ N11-098 } & \multicolumn{2}{|c|}{ N11-100 } \\
\hline & & $E W$ & Abund & $E W$ & Abund & $E W$ & Abund & $E W$ & Abund & $E W$ & Abund \\
\hline C II & 4267.00 & 150 & 7.60 & 69 & 7.63 & 116 & 7.31 & 140 & 7.71 & 56 & 7.65 \\
\hline N II & 3955.85 & - & - & - & - & - & - & - & - & - & - \\
\hline N II & 3995.00 & 20 & 6.65 & 70 & 7.46 & 48 & 7.53 & 48 & 7.00 & 80 & 7.77 \\
\hline N II & 4447.03 & - & - & - & - & 29 & 7.77 & - & - & 29 & 7.47 \\
\hline N II & 4601.48 & - & - & - & - & - & - & - & - & - & - \\
\hline N II & 4613.86 & - & - & - & - & - & - & - & - & - & - \\
\hline N II & 4630.54 & 20 & 6.94 & - & - & 42 & 7.75 & 35 & 7.04 & - & - \\
\hline N II & 4643.08 & - & - & - & - & - & - & - & - & - & - \\
\hline O II & 3912.00 & 22 & 8.22 & - & - & - & - & 49 & 8.42 & 53 & 8.20 \\
\hline O II & 3919.30 & - & - & - & - & - & - & - & - & - & - \\
\hline O II & 3945.04 & - & - & - & - & - & - & 32 & 8.40 & - & - \\
\hline O II & 3954.36 & 25 & 8.29 & - & - & - & - & 39 & 8.22 & 53 & 8.25 \\
\hline O II & 3982.71 & - & - & - & - & - & - & - & - & 33 & 8.31 \\
\hline O II & 4069.00 & 71 & 8.60 & - & - & - & - & 40 & 7.63 & 142 & 8.35 \\
\hline O II & 4072.15 & 46 & 8.54 & - & - & - & - & 44 & 7.95 & 86 & 8.18 \\
\hline O II & 4075.86 & 58 & 8.64 & - & - & 27 & 8.27 & 84 & 8.51 & 93 & 8.08 \\
\hline O II & 4078.84 & - & - & - & - & - & - & 15 & 8.01 & 26 & 8.16 \\
\hline O II & 4132.80 & - & - & - & - & - & - & 24 & 8.14 & 27 & 7.98 \\
\hline O II & 4156.53 & - & - & - & - & - & - & - & - & - & - \\
\hline O II & 4185.44 & - & - & - & - & - & - & - & - & 51 & 8.19 \\
\hline O II & 4317.00 & 32 & 8.48 & 81 & 8.62 & 15 & 8.19 & 47 & 8.38 & 70 & 8.54 \\
\hline O II & 4319.63 & 38 & 8.65 & 45 & 8.05 & - & - & 52 & 8.48 & 72 & 8.57 \\
\hline O II & 4325.76 & - & - & - & - & - & - & 19 & 8.50 & 37 & 8.88 \\
\hline O II & 4349.43 & - & - & - & - & - & - & 60 & 8.32 & 103 & 8.62 \\
\hline O II & 4351.00 & - & - & - & - & - & - & - & - & 63 & 8.02 \\
\hline O II & 4353.58 & - & - & - & - & - & - & - & - & - & - \\
\hline O II & 4366.00 & 28 & 8.32 & 79 & 8.51 & - & - & 48 & 8.33 & 48 & 8.17 \\
\hline O II & 4369.27 & - & - & - & - & - & - & - & - & 21 & 8.33 \\
\hline O II & 4395.94 & - & - & - & - & - & - & - & - & - & - \\
\hline O II & 4414.90 & 39 & 8.20 & - & - & - & - & 67 & 8.24 & 95 & 8.29 \\
\hline O II & 4416.98 & 30 & 8.28 & - & - & - & - & 57 & 8.39 & 79 & 8.40 \\
\hline O II & 4443.01 & - & - & - & - & - & - & - & - & - & - \\
\hline O II & 4452.38 & - & - & - & - & - & - & 23 & 8.41 & - & - \\
\hline O II & 4590.97 & 27 & 8.33 & 78 & 8.24 & - & - & 50 & 8.32 & 68 & 8.14 \\
\hline O II & 4596.18 & - & - & 72 & 8.24 & - & - & 43 & 8.26 & 61 & 8.17 \\
\hline O II & 4638.86 & - & - & - & - & - & - & 52 & 8.39 & 75 & 8.43 \\
\hline O II & 4641.81 & - & - & - & - & - & - & 74 & 8.31 & 101 & 8.28 \\
\hline O II & 4650.00 & 94 & 8.44 & - & - & 66 & 8.52 & 154 & 8.41 & - & - \\
\hline O II & 4661.63 & 32 & 8.40 & 72 & 8.23 & - & - & 51 & 8.31 & 82 & 8.44 \\
\hline O II & 4673.73 & - & - & - & - & - & - & 15 & 8.37 & - & - \\
\hline O II & 4676.23 & - & - & - & - & - & - & 38 & 8.17 & 83 & 8.58 \\
\hline O II & 4699.00 & - & - & - & - & - & - & 42 & 8.17 & 58 & 8.05 \\
\hline O II & 4705.35 & - & - & - & - & - & - & 32 & 8.05 & 61 & 8.12 \\
\hline O II & 4710.00 & - & - & - & - & - & - & - & - & - & - \\
\hline Mg II & 4481.00 & 125 & 6.99 & 44 & 6.69 & 154 & 7.14 & 112 & 7.03 & 70 & 7.10 \\
\hline Si II & 4128.05 & - & - & - & - & - & - & - & - & - & - \\
\hline Si II & 4130.89 & - & - & - & - & - & - & - & - & - & - \\
\hline Si III & 4552.62 & 78 & 7.13 & 130 & 7.17 & 59 & 7.22 & 123 & 7.23 & 122 & 7.16 \\
\hline Si III & 4567.84 & 60 & 7.08 & - & - & 38 & 6.97 & 97 & 7.17 & 96 & 7.13 \\
\hline Si III & 4574.75 & 43 & 7.26 & - & - & 20 & 6.89 & 65 & 7.24 & 64 & 7.31 \\
\hline Si IV & 4088.90 & - & - & - & - & - & - & - & - & - & - \\
\hline Si IV & 4116.10 & - & - & - & - & - & - & - & - & 93 & 7.19 \\
\hline Si IV & 4212.00 & - & - & - & - & - & - & - & - & - & - \\
\hline
\end{tabular}


I. Hunter et al.: Chemical compositions of B-type stars, Online Material p 42

Table 1. continued.

\begin{tabular}{|c|c|c|c|c|c|c|c|c|c|c|c|}
\hline \multirow[t]{2}{*}{ Species } & \multirow[t]{2}{*}{ Wavelength } & \multicolumn{2}{|c|}{ N11-101 } & \multicolumn{2}{|c|}{ N11-102 } & \multicolumn{2}{|c|}{ N11-104 } & \multicolumn{2}{|c|}{ N11-106 } & \multicolumn{2}{|c|}{ N11-108 } \\
\hline & & $E W$ & Abund & $E W$ & Abund & $E W$ & Abund & $E W$ & Abund & $E W$ & Abund \\
\hline C II & 4267.00 & 88 & 7.94 & 60 & 7.78 & 114 & 7.96 & 44 & 7.66 & 48 & 7.83 \\
\hline N II & 3955.85 & - & - & - & - & - & - & - & - & - & - \\
\hline N II & 3995.00 & $<28$ & $<7.09$ & $<48$ & $<7.58$ & - & - & 20 & 7.13 & 18 & 7.21 \\
\hline N II & 4447.03 & - & - & - & - & - & - & - & - & - & - \\
\hline N II & 4601.48 & - & - & - & - & - & - & - & - & - & - \\
\hline N II & 4613.86 & - & - & - & - & - & - & - & - & - & - \\
\hline N II & 4630.54 & - & - & - & - & $<39$ & $<7.20$ & - & - & - & - \\
\hline N II & 4643.08 & - & - & - & - & - & - & - & - & - & - \\
\hline O II & 3912.00 & 73 & 8.39 & - & - & 68 & 8.35 & 46 & 8.26 & 39 & 8.26 \\
\hline O II & 3919.30 & - & - & - & - & - & - & - & - & - & - \\
\hline O II & 3945.04 & 34 & 8.29 & - & - & 60 & 8.63 & 24 & 8.25 & 16 & 8.12 \\
\hline O II & 3954.36 & 64 & 8.33 & - & - & 57 & 8.23 & 43 & 8.25 & 31 & 8.16 \\
\hline O II & 3982.71 & - & - & - & - & - & - & 24 & 8.26 & 22 & 8.32 \\
\hline O II & 4069.00 & 233 & 8.86 & - & - & - & - & 159 & 8.65 & - & - \\
\hline O II & 4072.15 & 136 & 8.50 & - & - & - & - & 74 & 8.19 & 62 & 8.14 \\
\hline O II & 4075.86 & 116 & 8.12 & - & - & 85 & 8.08 & 99 & 8.29 & 78 & 8.17 \\
\hline O II & 4078.84 & - & - & - & - & - & - & 22 & 8.21 & 14 & 8.09 \\
\hline O II & 4132.80 & 56 & 8.41 & - & - & 50 & 8.39 & 35 & 8.30 & 30 & 8.29 \\
\hline O II & 4156.53 & - & - & - & - & - & - & - & - & - & - \\
\hline O II & 4185.44 & - & - & - & - & - & - & 28 & 7.94 & - & - \\
\hline O II & 4317.00 & 52 & 8.34 & - & - & - & - & - & - & - & - \\
\hline O II & 4319.63 & 66 & 8.48 & - & - & - & - & - & - & - & - \\
\hline O II & 4325.76 & - & - & - & - & - & - & 30 & 9.00 & - & - \\
\hline O II & 4349.43 & 97 & 8.45 & - & - & - & - & - & - & - & - \\
\hline O II & 4351.00 & 60 & 7.92 & - & - & - & - & 52 & 7.99 & 27 & 7.69 \\
\hline O II & 4353.58 & - & - & - & - & - & - & - & - & - & - \\
\hline O II & 4366.00 & 61 & 8.35 & - & - & 67 & 8.36 & - & - & - & - \\
\hline O II & 4369.27 & - & - & - & - & - & - & - & - & - & - \\
\hline O II & 4395.94 & - & - & - & - & - & - & - & - & - & - \\
\hline O II & 4414.90 & 87 & 8.16 & 85 & 8.45 & - & - & 79 & 8.39 & 53 & 8.27 \\
\hline O II & 4416.98 & 69 & 8.24 & 49 & 8.26 & - & - & 63 & 8.46 & 51 & 8.47 \\
\hline O II & 4443.01 & - & - & - & - & - & - & - & - & - & - \\
\hline O II & 4452.38 & - & - & - & - & - & - & - & - & - & - \\
\hline O II & 4590.97 & 87 & 8.25 & 70 & 8.43 & 72 & 8.21 & 64 & 8.28 & - & - \\
\hline O II & 4596.18 & 62 & 8.12 & 43 & 8.10 & 57 & 8.08 & 52 & 8.24 & 37 & 8.16 \\
\hline O II & 4638.86 & 82 & 8.46 & - & - & 74 & 8.36 & 65 & 8.57 & 52 & 8.56 \\
\hline O II & 4641.81 & 131 & 8.42 & - & - & 107 & 8.35 & 111 & 8.62 & 89 & 8.58 \\
\hline O II & 4650.00 & - & - & - & - & 243 & 8.60 & - & - & - & - \\
\hline O II & 4661.63 & 78 & 8.36 & - & - & 72 & 8.26 & 74 & 8.60 & 62 & 8.61 \\
\hline O II & 4673.73 & - & - & - & - & - & - & - & - & - & - \\
\hline O II & 4676.23 & - & - & - & - & - & - & 50 & 8.45 & 37 & 8.42 \\
\hline O II & 4699.00 & 79 & 8.25 & - & - & 55 & 7.96 & 58 & 8.22 & - & - \\
\hline O II & 4705.35 & 60 & 8.09 & - & - & 57 & 8.08 & 63 & 8.31 & 48 & 8.22 \\
\hline O II & 4710.00 & - & - & - & - & - & - & - & - & - & - \\
\hline Mg II & 4481.00 & 86 & 7.21 & 74 & 7.25 & 89 & 7.15 & 73 & 7.19 & 64 & 7.14 \\
\hline Si II & 4128.05 & - & - & - & - & - & - & - & - & - & - \\
\hline Si II & 4130.89 & - & - & - & - & - & - & - & - & - & - \\
\hline Si III & 4552.62 & 156 & 7.14 & 85 & 7.20 & 129 & 7.21 & 106 & 7.18 & 91 & 7.21 \\
\hline Si III & 4567.84 & 126 & 7.19 & - & - & 108 & 7.22 & 87 & 7.24 & 66 & 7.17 \\
\hline Si III & 4574.75 & 61 & 7.15 & - & - & 75 & 7.31 & 38 & 7.14 & 29 & 7.14 \\
\hline Si IV & 4088.90 & - & - & - & - & - & - & - & - & - & - \\
\hline Si IV & 4116.10 & 146 & 7.17 & - & - & - & - & 145 & 7.37 & 148 & 7.43 \\
\hline Si IV & 4212.00 & - & - & - & - & - & - & 37 & 7.13 & - & - \\
\hline
\end{tabular}


Table 1. continued.

\begin{tabular}{|c|c|c|c|c|c|c|c|c|c|c|c|}
\hline \multirow[t]{2}{*}{ Species } & \multirow[t]{2}{*}{ Wavelength } & \multicolumn{2}{|c|}{ N11-109 } & \multicolumn{2}{|c|}{ N11-110 } & \multicolumn{2}{|c|}{ N11-111 } & \multicolumn{2}{|c|}{ N11-114 } & \multicolumn{2}{|c|}{ N11-115 } \\
\hline & & $E W$ & Abund & $E W$ & Abund & $E W$ & Abund & $E W$ & Abund & $E W$ & Abund \\
\hline C II & 4267.00 & 82 & 7.75 & 121 & 7.79 & 108 & 7.67 & - & - & 89 & 7.63 \\
\hline N II & 3955.85 & - & - & - & - & - & - & - & - & - & - \\
\hline N II & 3995.00 & 55 & 7.24 & 108 & 7.45 & 43 & 6.87 & $<54$ & $<7.92$ & 34 & 6.82 \\
\hline N II & 4447.03 & - & - & 43 & 7.33 & - & - & - & - & - & - \\
\hline N II & 4601.48 & - & - & - & - & - & - & - & - & - & - \\
\hline N II & 4613.86 & - & - & - & - & - & - & - & - & - & - \\
\hline N II & 4630.54 & - & - & 78 & 7.38 & 37 & 7.00 & - & - & 29 & 6.96 \\
\hline N II & 4643.08 & - & - & - & - & - & - & - & - & - & - \\
\hline O II & 3912.00 & 86 & 8.35 & 92 & 8.46 & 72 & 8.51 & - & - & 74 & 8.51 \\
\hline O II & 3919.30 & - & - & - & - & - & - & - & - & - & - \\
\hline O II & 3945.04 & 52 & 8.33 & 63 & 8.46 & 53 & 8.58 & - & - & 51 & 8.55 \\
\hline O II & 3954.36 & 75 & 8.21 & 69 & 8.19 & 77 & 8.59 & - & - & 67 & 8.46 \\
\hline O II & 3982.71 & 40 & 8.16 & 69 & 8.53 & - & - & - & - & - & - \\
\hline O II & 4069.00 & 136 & 8.08 & 181 & 8.47 & 186 & 8.94 & - & - & 149 & 8.63 \\
\hline O II & 4072.15 & 107 & 8.05 & 120 & 8.34 & 113 & 8.79 & - & - & 101 & 8.69 \\
\hline O II & 4075.86 & 184 & 8.45 & 143 & 8.37 & 114 & 8.60 & - & - & 108 & 8.59 \\
\hline O II & 4078.84 & - & - & 47 & 8.40 & 44 & 8.61 & - & - & 46 & 8.66 \\
\hline O II & 4132.80 & - & - & 53 & 8.29 & 36 & 8.21 & - & - & 52 & 8.52 \\
\hline O II & 4156.53 & - & - & - & - & - & - & - & - & - & - \\
\hline O II & 4185.44 & - & - & - & - & - & - & - & - & - & - \\
\hline O II & 4317.00 & - & - & 116 & 8.71 & 86 & 8.77 & - & - & 83 & 8.79 \\
\hline O II & 4319.63 & - & - & 121 & 8.76 & 98 & 8.95 & - & - & 76 & 8.67 \\
\hline O II & 4325.76 & - & - & 43 & 8.67 & - & - & - & - & - & - \\
\hline O II & 4349.43 & - & - & 155 & 8.64 & - & - & - & - & 86 & 8.47 \\
\hline O II & 4351.00 & 83 & 8.02 & 107 & 8.39 & - & - & - & - & 74 & 8.35 \\
\hline O II & 4353.58 & - & - & - & - & - & - & - & - & - & - \\
\hline O II & 4366.00 & - & - & 115 & 8.60 & 84 & 8.65 & - & - & 76 & 8.60 \\
\hline O II & 4369.27 & - & - & 28 & 8.36 & - & - & - & - & - & - \\
\hline O II & 4395.94 & - & - & - & - & - & - & - & - & - & - \\
\hline O II & 4414.90 & 171 & 8.47 & 161 & 8.52 & 113 & 8.53 & - & - & 107 & 8.47 \\
\hline O II & 4416.98 & 119 & 8.41 & 129 & 8.55 & 80 & 8.45 & - & - & 98 & 8.69 \\
\hline O II & 4443.01 & - & - & - & - & - & - & - & - & - & - \\
\hline O II & 4452.38 & - & - & 57 & 8.62 & 60 & 8.95 & - & - & - & - \\
\hline O II & 4590.97 & 121 & 8.29 & 120 & 8.48 & 85 & 8.54 & - & - & 86 & 8.56 \\
\hline O II & 4596.18 & 106 & 8.29 & - & - & 89 & 8.62 & - & - & 85 & 8.56 \\
\hline O II & 4638.86 & 106 & 8.44 & 117 & 8.54 & 90 & 8.67 & - & - & 79 & 8.53 \\
\hline O II & 4641.81 & 211 & 8.60 & 175 & 8.52 & 128 & 8.65 & - & - & 111 & 8.51 \\
\hline O II & 4650.00 & - & - & - & - & - & - & - & - & - & - \\
\hline O II & 4661.63 & 139 & 8.57 & 133 & 8.59 & 96 & 8.67 & - & - & 77 & 8.43 \\
\hline O II & 4673.73 & - & - & - & - & 35 & 8.72 & - & - & 30 & 8.59 \\
\hline O II & 4676.23 & 110 & 8.51 & 98 & 8.43 & 91 & 8.74 & - & - & 74 & 8.52 \\
\hline O II & 4699.00 & - & - & 92 & 8.28 & 86 & 8.47 & - & - & 96 & 8.50 \\
\hline O II & 4705.35 & 84 & 8.17 & 95 & 8.38 & 80 & 8.54 & - & - & 67 & 8.33 \\
\hline O II & 4710.00 & - & - & - & - & - & - & - & - & 36 & 8.85 \\
\hline Mg II & 4481.00 & 57 & 6.84 & 109 & 7.06 & 92 & 6.95 & 64 & 7.19 & 69 & 6.85 \\
\hline Si II & 4128.05 & - & - & - & - & - & - & - & - & - & - \\
\hline Si II & 4130.89 & - & - & - & - & - & - & - & - & - & - \\
\hline Si III & 4552.62 & 260 & 7.25 & 214 & 7.14 & 146 & 7.18 & 75 & 7.20 & 124 & 7.15 \\
\hline Si III & 4567.84 & 199 & 7.19 & 194 & 7.27 & 121 & 7.17 & - & - & 106 & 7.19 \\
\hline Si III & 4574.75 & 99 & 7.09 & 124 & 7.27 & 81 & 7.23 & - & - & 75 & 7.31 \\
\hline Si IV & 4088.90 & - & - & - & - & - & - & - & - & - & - \\
\hline Si IV & 4116.10 & 177 & 7.32 & 64 & 7.28 & - & - & - & - & - & - \\
\hline Si IV & 4212.00 & - & - & - & - & - & - & - & - & - & - \\
\hline
\end{tabular}


I. Hunter et al.: Chemical compositions of B-type stars, Online Material p 44

Table 1. continued.

\begin{tabular}{|c|c|c|c|c|c|c|c|c|c|c|c|}
\hline \multirow[t]{2}{*}{ Species } & \multirow[t]{2}{*}{ Wavelength } & \multicolumn{2}{|c|}{ N11-116 } & \multicolumn{2}{|c|}{ N11-117 } & \multicolumn{2}{|c|}{ N11-118 } & \multicolumn{2}{|c|}{ N11-120 } & \multicolumn{2}{|c|}{ N11-121 } \\
\hline & & $E W$ & Abund & $E W$ & Abund & $E W$ & Abund & $E W$ & Abund & $E W$ & Abund \\
\hline C II & 4267.00 & 145 & 7.74 & 56 & 7.46 & 113 & 7.93 & 62 & 7.90 & 57 & 7.49 \\
\hline N II & 3955.85 & - & - & - & - & - & - & - & - & - & - \\
\hline N II & 3995.00 & 42 & 6.86 & $<40$ & $<7.06$ & 62 & 7.28 & $<41$ & $<7.56$ & $<67$ & $<7.48$ \\
\hline N II & 4447.03 & - & - & - & - & 38 & 7.41 & - & - & - & - \\
\hline N II & 4601.48 & - & - & - & - & - & - & - & - & - & - \\
\hline N II & 4613.86 & - & - & - & - & - & - & - & - & - & - \\
\hline N II & 4630.54 & 42 & 7.09 & - & - & 59 & 7.48 & - & - & - & - \\
\hline N II & 4643.08 & - & - & - & - & - & - & - & - & - & - \\
\hline O II & 3912.00 & 46 & 8.25 & - & - & 50 & 8.11 & - & - & - & - \\
\hline O II & 3919.30 & - & - & - & - & - & - & - & - & - & - \\
\hline O II & 3945.04 & - & - & - & - & - & - & - & - & - & - \\
\hline O II & 3954.36 & - & - & 49 & 8.23 & 61 & 8.28 & - & - & - & - \\
\hline O II & 3982.71 & - & - & - & - & - & - & - & - & - & - \\
\hline O II & 4069.00 & - & - & - & - & - & - & - & - & - & - \\
\hline O II & 4072.15 & - & - & - & - & - & - & - & - & - & - \\
\hline O II & 4075.86 & - & - & - & - & - & - & - & - & - & - \\
\hline O II & 4078.84 & - & - & - & - & - & - & - & - & - & - \\
\hline O II & 4132.80 & - & - & - & - & - & - & - & - & - & - \\
\hline O II & 4156.53 & - & - & - & - & - & - & - & - & - & - \\
\hline O II & 4185.44 & - & - & - & - & - & - & - & - & - & - \\
\hline O II & 4317.00 & 65 & 8.57 & - & - & 53 & 8.17 & - & - & - & - \\
\hline O II & 4319.63 & 57 & 8.42 & - & - & 75 & 8.52 & - & - & - & - \\
\hline O II & 4325.76 & - & - & - & - & - & - & - & - & - & - \\
\hline O II & 4349.43 & - & - & - & - & - & - & - & - & - & - \\
\hline O II & 4351.00 & - & - & - & - & - & - & - & - & - & - \\
\hline O II & 4353.58 & - & - & - & - & - & - & - & - & - & - \\
\hline O II & 4366.00 & 69 & 8.56 & - & - & 74 & 8.43 & - & - & - & - \\
\hline O II & 4369.27 & - & - & - & - & 33 & 8.53 & - & - & - & - \\
\hline O II & 4395.94 & - & - & - & - & - & - & - & - & - & - \\
\hline O II & 4414.90 & - & - & - & - & 76 & 8.00 & - & - & - & - \\
\hline O II & 4416.98 & - & - & - & - & 105 & 8.63 & - & - & - & - \\
\hline O II & 4443.01 & - & - & - & - & - & - & - & - & - & - \\
\hline O II & 4452.38 & - & - & - & - & - & - & - & - & - & - \\
\hline O II & 4590.97 & 53 & 8.21 & - & - & 60 & 8.03 & 78 & 8.55 & - & - \\
\hline O II & 4596.18 & 61 & 8.41 & - & - & 60 & 8.11 & 65 & 8.46 & - & - \\
\hline O II & 4638.86 & 59 & 8.36 & - & - & 83 & 8.46 & - & - & - & - \\
\hline O II & 4641.81 & 94 & 8.40 & - & - & 120 & 8.45 & - & - & - & - \\
\hline O II & 4650.00 & 202 & 8.55 & - & - & 243 & 8.54 & - & - & 213 & 8.56 \\
\hline O II & 4661.63 & 66 & 8.40 & 74 & 8.46 & 66 & 8.15 & 60 & 8.51 & - & - \\
\hline O II & 4673.73 & - & - & - & - & - & - & - & - & - & - \\
\hline O II & 4676.23 & - & - & - & - & - & - & - & - & - & - \\
\hline O II & 4699.00 & 65 & 8.38 & - & - & 51 & 7.92 & - & - & - & - \\
\hline O II & 4705.35 & 46 & 8.20 & - & - & 43 & 7.85 & - & - & - & - \\
\hline O II & 4710.00 & - & - & - & - & - & - & - & - & - & - \\
\hline Mg II & 4481.00 & 115 & 7.05 & 60 & 6.89 & 104 & 7.25 & 67 & 7.17 & 55 & 6.84 \\
\hline Si II & 4128.05 & - & - & - & - & - & - & - & - & - & - \\
\hline Si II & 4130.89 & - & - & - & - & - & - & - & - & - & - \\
\hline Si III & 4552.62 & 130 & 7.10 & 110 & 7.19 & 136 & 7.18 & 88 & 7.18 & 74 & 6.64 \\
\hline Si III & 4567.84 & 115 & 7.20 & - & - & 112 & 7.17 & - & - & - & - \\
\hline Si III & 4574.75 & 72 & 7.18 & - & - & 79 & 7.30 & - & - & - & - \\
\hline Si IV & 4088.90 & - & - & - & - & - & - & - & - & - & - \\
\hline Si IV & 4116.10 & - & - & - & - & - & - & - & - & - & - \\
\hline Si IV & 4212.00 & - & - & - & - & - & - & - & - & - & - \\
\hline
\end{tabular}


I. Hunter et al.: Chemical compositions of B-type stars, Online Material p 45

Table 1. continued.

\begin{tabular}{|c|c|c|c|c|c|c|c|c|c|c|c|}
\hline \multirow[t]{2}{*}{ Species } & \multirow[t]{2}{*}{ Wavelength } & \multicolumn{2}{|c|}{ N11-122 } & \multicolumn{2}{|c|}{ N11-124 } & \multicolumn{2}{|c|}{ NGC 2004-003 } & \multicolumn{2}{|c|}{ NGC 2004-010 } & \multicolumn{2}{|c|}{ NGC 2004-011 } \\
\hline & & $E W$ & Abund & $E W$ & Abund & $E W$ & Abund & $E W$ & Abund & $E W$ & Abund \\
\hline C II & 4267.00 & - & - & 78 & 7.80 & 173 & 7.48 & 194 & 7.42 & 130 & 7.63 \\
\hline $\mathrm{N}$ II & 3955.85 & - & - & - & - & 21 & 7.89 & 64 & 8.19 & 52 & 7.85 \\
\hline N II & 3995.00 & $<34$ & $<7.72$ & 43 & 7.25 & 83 & 7.79 & 202 & 8.11 & 196 & 7.76 \\
\hline N II & 4447.03 & - & - & - & - & 31 & 7.81 & 93 & 8.04 & 76 & 7.60 \\
\hline N II & 4601.48 & - & - & - & - & 31 & 7.86 & 97 & 8.17 & 95 & 7.85 \\
\hline $\mathrm{N}$ II & 4613.86 & - & - & - & - & 19 & 7.80 & 65 & 8.11 & 60 & 7.81 \\
\hline N II & 4630.54 & - & - & - & - & 60 & 7.84 & 180 & 8.21 & 153 & 7.68 \\
\hline N II & 4643.08 & - & - & - & - & 34 & 7.93 & 108 & 8.26 & 58 & 7.55 \\
\hline O II & 3912.00 & - & - & 65 & 8.38 & 12 & 8.38 & 29 & 8.24 & 84 & 8.32 \\
\hline O II & 3919.30 & - & - & - & - & - & - & - & - & - & - \\
\hline O II & 3945.04 & - & - & 37 & 8.34 & 8 & 8.30 & 19 & 8.18 & 57 & 8.30 \\
\hline O II & 3954.36 & - & - & 43 & 8.13 & 15 & 8.33 & 30 & 8.12 & 86 & 8.22 \\
\hline O II & 3982.71 & - & - & - & - & 8 & 8.30 & 20 & 8.21 & 75 & 8.46 \\
\hline O II & 4069.00 & - & - & 116 & 8.24 & 29 & 8.35 & 62 & 8.14 & 188 & 8.33 \\
\hline O II & 4072.15 & - & - & 58 & 7.90 & 28 & 8.43 & 74 & 8.42 & 181 & 8.51 \\
\hline O II & 4075.86 & - & - & 81 & 8.10 & 37 & 8.47 & 69 & 8.19 & 190 & 8.37 \\
\hline O II & 4078.84 & - & - & 31 & 8.28 & - & - & - & - & 31 & 8.04 \\
\hline O II & 4132.80 & - & - & - & - & - & - & - & - & 60 & 8.33 \\
\hline O II & 4156.53 & - & - & - & - & - & - & - & - & 15 & 8.32 \\
\hline O II & 4185.44 & - & - & - & - & - & - & - & - & - & - \\
\hline O II & 4317.00 & - & - & 37 & 8.02 & 19 & 8.48 & 43 & 8.38 & 133 & 8.60 \\
\hline O II & 4319.63 & - & - & 41 & 8.07 & 23 & 8.62 & 43 & 8.38 & 142 & 8.66 \\
\hline O II & 4325.76 & - & - & - & - & - & - & 14 & 8.44 & 34 & 8.44 \\
\hline O II & 4349.43 & - & - & 54 & 8.05 & - & - & 74 & 8.43 & 227 & 8.73 \\
\hline O II & 4351.00 & - & - & - & - & - & - & 24 & 8.02 & 87 & 8.12 \\
\hline O II & 4353.58 & - & - & - & - & - & - & - & - & - & - \\
\hline O II & 4366.00 & - & - & 34 & 7.91 & 18 & 8.37 & 36 & 8.18 & 127 & 8.47 \\
\hline O II & 4369.27 & - & - & - & - & - & - & - & - & - & - \\
\hline O II & 4395.94 & - & - & - & - & - & - & 29 & 8.77 & 45 & 8.42 \\
\hline O II & 4414.90 & - & - & 78 & 8.16 & 32 & 8.41 & 65 & 8.22 & 223 & 8.59 \\
\hline O II & 4416.98 & - & - & 56 & 8.14 & 30 & 8.61 & 46 & 8.22 & 154 & 8.48 \\
\hline O II & 4443.01 & - & - & - & - & - & - & - & - & - & - \\
\hline O II & 4452.38 & - & - & - & - & 10 & 8.68 & 19 & 8.43 & 54 & 8.50 \\
\hline O II & 4590.97 & - & - & 51 & 7.99 & 17 & 8.48 & 36 & 8.19 & 137 & 8.36 \\
\hline O II & 4596.18 & - & - & 52 & 8.09 & 16 & 8.55 & 30 & 8.19 & 109 & 8.29 \\
\hline O II & 4638.86 & - & - & 57 & 8.25 & 20 & 8.49 & 42 & 8.29 & 155 & 8.58 \\
\hline O II & 4641.81 & - & - & 71 & 8.02 & 37 & 8.48 & 75 & 8.28 & 272 & 8.70 \\
\hline O II & 4650.00 & - & - & - & - & - & - & - & - & - & - \\
\hline O II & 4661.63 & - & - & 52 & 8.10 & - & - & 51 & 8.35 & 170 & 8.58 \\
\hline O II & 4673.73 & - & - & - & - & - & - & 11 & 8.37 & 41 & 8.53 \\
\hline O II & 4676.23 & - & - & 42 & 8.05 & 21 & 8.59 & 41 & 8.33 & 154 & 8.63 \\
\hline O II & 4699.00 & - & - & - & - & 8 & 8.21 & 20 & 8.01 & 64 & 8.02 \\
\hline O II & 4705.35 & 42 & 8.29 & - & - & 11 & 8.37 & 26 & 8.13 & 82 & 8.15 \\
\hline O II & 4710.00 & - & - & - & - & - & - & 15 & 8.71 & 23 & 8.31 \\
\hline Mg II & 4481.00 & 70 & 7.27 & 58 & 6.97 & 302 & 7.02 & 228 & 7.11 & 136 & 7.15 \\
\hline Si II & 4128.05 & - & - & - & - & 133 & 7.34 & 84 & 7.35 & - & - \\
\hline Si II & 4130.89 & - & - & - & - & 144 & 7.21 & 91 & 7.20 & - & - \\
\hline Si III & 4552.62 & 67 & 7.22 & 87 & 6.94 & 107 & 7.23 & 205 & 7.22 & 350 & 7.21 \\
\hline Si III & 4567.84 & - & - & 69 & 6.92 & 77 & 7.18 & 161 & 7.21 & 286 & 7.20 \\
\hline Si III & 4574.75 & - & - & 41 & 6.96 & 44 & 7.24 & 89 & 7.18 & 166 & 7.17 \\
\hline Si IV & 4088.90 & - & - & - & - & - & - & - & - & - & - \\
\hline Si IV & 4116.10 & - & - & 47 & 6.97 & - & - & - & - & 87 & 7.22 \\
\hline Si IV & 4212.00 & - & - & - & - & - & - & - & - & - & - \\
\hline
\end{tabular}


I. Hunter et al.: Chemical compositions of B-type stars, Online Material p 46

Table 1. continued.

\begin{tabular}{|c|c|c|c|c|c|c|c|c|c|c|c|}
\hline \multirow[t]{2}{*}{ Species } & \multirow[t]{2}{*}{ Wavelength } & \multicolumn{2}{|c|}{ NGC 2004-012 } & \multicolumn{2}{|c|}{ NGC 2004-013 } & \multicolumn{2}{|c|}{ NGC 2004-014 } & \multicolumn{2}{|c|}{ NGC 2004-015 } & \multicolumn{2}{|c|}{ NGC 2004-020 } \\
\hline & & $E W$ & Abund & $E W$ & Abund & $E W$ & Abund & $E W$ & Abund & $E W$ & Abund \\
\hline C II & 4267.00 & 134 & 7.62 & 146 & 7.77 & 191 & 7.50 & 176 & 8.05 & 187 & 8.14 \\
\hline N II & 3955.85 & 48 & 7.80 & - & - & 19 & 7.56 & - & - & - & - \\
\hline N II & 3995.00 & 177 & 7.69 & 42 & 6.84 & 82 & 7.47 & 58 & 6.97 & 105 & 7.37 \\
\hline N II & 4447.03 & 69 & 7.54 & - & - & 33 & 7.49 & - & - & 72 & 7.60 \\
\hline N II & 4601.48 & 105 & 7.94 & - & - & 30 & 7.55 & - & - & - & - \\
\hline N II & 4613.86 & 56 & 7.77 & - & - & 17 & 7.46 & - & - & - & - \\
\hline N II & 4630.54 & 152 & 7.71 & 28 & 6.86 & 59 & 7.51 & 32 & 6.94 & 88 & 7.41 \\
\hline N II & 4643.08 & 83 & 7.77 & - & - & 34 & 7.63 & - & - & - & - \\
\hline O II & 3912.00 & 86 & 8.39 & 56 & 8.35 & 23 & 8.23 & 74 & 8.19 & - & - \\
\hline O II & 3919.30 & - & - & - & - & - & - & - & - & - & - \\
\hline O II & 3945.04 & 56 & 8.34 & 38 & 8.35 & 18 & 8.31 & 51 & 8.21 & 49 & 8.23 \\
\hline O II & 3954.36 & 84 & 8.27 & 48 & 8.19 & 25 & 8.19 & 86 & 8.21 & 70 & 8.13 \\
\hline O II & 3982.71 & 55 & 8.32 & - & - & 10 & 8.02 & - & - & - & - \\
\hline O II & 4069.00 & 189 & 8.43 & - & - & 48 & 8.15 & - & - & - & - \\
\hline O II & 4072.15 & 170 & 8.55 & - & - & 39 & 8.15 & - & - & - & - \\
\hline O II & 4075.86 & 173 & 8.38 & - & - & 55 & 8.27 & - & - & - & - \\
\hline O II & 4078.84 & 37 & 8.19 & - & - & - & - & - & - & - & - \\
\hline O II & 4132.80 & 71 & 8.48 & - & - & - & - & - & - & - & - \\
\hline O II & 4156.53 & 21 & 8.51 & - & - & - & - & - & - & - & - \\
\hline O II & 4185.44 & - & - & - & - & - & - & - & - & - & - \\
\hline O II & 4317.00 & 117 & 8.57 & 56 & 8.35 & 29 & 8.30 & 98 & 8.33 & 93 & 8.37 \\
\hline O II & 4319.63 & 127 & 8.64 & 72 & 8.61 & 32 & 8.38 & 142 & 8.64 & 87 & 8.31 \\
\hline O II & 4325.76 & 33 & 8.47 & - & - & - & - & - & - & - & - \\
\hline O II & 4349.43 & 193 & 8.67 & - & - & 48 & 8.38 & - & - & - & - \\
\hline O II & 4351.00 & 88 & 8.20 & - & - & 17 & 8.06 & - & - & - & - \\
\hline O II & 4353.58 & - & - & - & - & - & - & - & - & - & - \\
\hline O II & 4366.00 & 122 & 8.52 & 49 & 8.15 & 25 & 8.12 & 117 & 8.38 & 88 & 8.23 \\
\hline O II & 4369.27 & 13 & 8.03 & - & - & - & - & 50 & 8.61 & - & - \\
\hline O II & 4395.94 & 45 & 8.46 & - & - & - & - & - & - & - & - \\
\hline O II & 4414.90 & 188 & 8.52 & - & - & 44 & 8.16 & - & - & - & - \\
\hline O II & 4416.98 & 143 & 8.51 & - & - & 38 & 8.31 & - & - & - & - \\
\hline O II & 4443.01 & - & - & - & - & - & - & - & - & - & - \\
\hline O II & 4452.38 & 54 & 8.55 & 27 & 8.36 & 17 & 8.55 & - & - & - & - \\
\hline O II & 4590.97 & 124 & 8.39 & 56 & 8.18 & 20 & 8.07 & 108 & 8.16 & 88 & 8.10 \\
\hline O II & 4596.18 & 103 & 8.34 & 48 & 8.13 & 19 & 8.15 & 75 & 8.03 & 77 & 8.11 \\
\hline O II & 4638.86 & 139 & 8.58 & - & - & 36 & 8.44 & - & - & - & - \\
\hline O II & 4641.81 & 229 & 8.62 & - & - & 57 & 8.35 & - & - & - & - \\
\hline O II & 4650.00 & - & - & - & - & - & - & - & - & - & - \\
\hline O II & 4661.63 & 152 & 8.58 & 66 & 8.32 & 35 & 8.34 & 143 & 8.42 & 111 & 8.31 \\
\hline O II & 4673.73 & 33 & 8.46 & - & - & - & - & - & - & - & - \\
\hline O II & 4676.23 & 131 & 8.58 & - & - & 30 & 8.37 & - & - & - & - \\
\hline O II & 4699.00 & 69 & 8.11 & - & - & 16 & 8.07 & - & - & - & - \\
\hline O II & 4705.35 & 87 & 8.26 & - & - & 19 & 8.16 & - & - & - & - \\
\hline O II & 4710.00 & 32 & 8.53 & - & - & - & - & - & - & - & - \\
\hline Mg II & 4481.00 & 132 & 7.08 & 130 & 7.19 & 214 & 7.06 & 151 & 7.25 & 156 & 7.32 \\
\hline Si II & 4128.05 & - & - & - & - & 80 & 7.34 & - & - & - & - \\
\hline Si II & 4130.89 & - & - & - & - & 89 & 7.22 & - & - & - & - \\
\hline Si III & 4552.62 & 308 & 7.18 & 164 & 7.40 & 146 & 7.24 & 311 & 7.16 & 269 & 7.23 \\
\hline Si III & 4567.84 & 256 & 7.20 & 122 & 7.22 & 112 & 7.21 & 251 & 7.14 & 222 & 7.23 \\
\hline Si III & 4574.75 & 151 & 7.18 & 61 & 6.94 & 60 & 7.15 & 156 & 7.19 & 134 & 7.20 \\
\hline Si IV & 4088.90 & - & - & - & - & - & - & - & - & - & - \\
\hline Si IV & 4116.10 & 69 & 7.21 & - & - & - & - & - & - & - & - \\
\hline Si IV & 4212.00 & - & - & - & - & - & - & - & - & - & - \\
\hline
\end{tabular}


I. Hunter et al.: Chemical compositions of B-type stars, Online Material p 47

Table 1. continued.

\begin{tabular}{|c|c|c|c|c|c|c|c|c|c|c|c|}
\hline \multirow[t]{2}{*}{ Species } & \multirow[t]{2}{*}{ Wavelength } & \multicolumn{2}{|c|}{ NGC 2004-021 } & \multicolumn{2}{|c|}{ NGC 2004-022 } & \multicolumn{2}{|c|}{ NGC 2004-024 } & \multicolumn{2}{|c|}{ NGC 2004-026 } & \multicolumn{2}{|c|}{ NGC 2004-029 } \\
\hline & & $E W$ & Abund & $E W$ & Abund & $E W$ & Abund & $E W$ & Abund & $E W$ & Abund \\
\hline C II & 4267.00 & 167 & 7.74 & 119 & 7.53 & 43 & 7.18 & 131 & 7.82 & 112 & 7.73 \\
\hline N II & 3955.85 & 18 & 7.26 & 38 & 7.70 & - & - & - & - & - & - \\
\hline N II & 3995.00 & 82 & 7.07 & 149 & 7.58 & 282 & 8.13 & - & - & 41 & 6.87 \\
\hline N II & 4447.03 & 22 & 6.93 & 68 & 7.54 & 124 & 7.91 & - & - & 12 & 6.75 \\
\hline N II & 4601.48 & 42 & 7.37 & 88 & 7.86 & - & - & 21 & 7.20 & - & - \\
\hline N II & 4613.86 & 30 & 7.42 & 55 & 7.79 & - & - & 10 & 7.03 & - & - \\
\hline N II & 4630.54 & 59 & 7.13 & 118 & 7.56 & 273 & 8.24 & 27 & 6.90 & 23 & 6.80 \\
\hline N II & 4643.08 & - & - & 73 & 7.73 & - & - & 12 & 6.90 & 10 & 6.82 \\
\hline O II & 3912.00 & 94 & 8.43 & 94 & 8.51 & 65 & 8.07 & 42 & 8.23 & 48 & 8.20 \\
\hline O II & 3919.30 & - & - & - & - & - & - & - & - & - & - \\
\hline O II & 3945.04 & 67 & 8.45 & 59 & 8.44 & - & - & 26 & 8.19 & 37 & 8.33 \\
\hline O II & 3954.36 & 88 & 8.30 & 86 & 8.37 & 144 & 8.50 & 35 & 8.09 & 52 & 8.27 \\
\hline O II & 3982.71 & - & - & 59 & 8.44 & - & - & 23 & 8.15 & 32 & 8.24 \\
\hline O II & 4069.00 & 197 & 8.44 & 192 & 8.54 & - & - & 87 & 8.22 & 107 & 8.27 \\
\hline O II & 4072.15 & 159 & 8.41 & 153 & 8.55 & - & - & 51 & 8.06 & 74 & 8.30 \\
\hline O II & 4075.86 & 182 & 8.37 & 157 & 8.39 & - & - & 63 & 8.12 & 80 & 8.21 \\
\hline O II & 4078.84 & 42 & 8.26 & 41 & 8.31 & - & - & 20 & 8.14 & 27 & 8.23 \\
\hline O II & 4132.80 & 54 & 8.26 & 58 & 8.38 & - & - & 27 & 8.16 & 29 & 8.09 \\
\hline O II & 4156.53 & 20 & 8.48 & 28 & 8.70 & - & - & 9 & 8.13 & - & - \\
\hline O II & 4185.44 & - & - & - & - & - & - & - & - & - & - \\
\hline O II & 4317.00 & 128 & 8.63 & 120 & 8.70 & - & - & 44 & 8.27 & 64 & 8.50 \\
\hline O II & 4319.63 & 135 & 8.67 & 119 & 8.69 & - & - & 47 & 8.33 & 67 & 8.55 \\
\hline O II & 4325.76 & 38 & 8.54 & 35 & 8.56 & - & - & 13 & 8.20 & 19 & 8.32 \\
\hline O II & 4349.43 & 198 & 8.67 & 183 & 8.77 & - & - & 56 & 8.23 & 75 & 8.33 \\
\hline O II & 4351.00 & 86 & 8.19 & 100 & 8.40 & - & - & 35 & 8.01 & 44 & 8.00 \\
\hline O II & 4353.58 & - & - & 24 & 8.52 & - & - & - & - & - & - \\
\hline O II & 4366.00 & 136 & 8.59 & 124 & 8.63 & - & - & 46 & 8.26 & 63 & 8.42 \\
\hline O II & 4369.27 & 14 & 8.06 & 24 & 8.34 & - & - & 11 & 8.08 & 16 & 8.18 \\
\hline O II & 4395.94 & 53 & 8.55 & 48 & 8.55 & - & - & 30 & 8.56 & 36 & 8.56 \\
\hline O II & 4414.90 & 201 & 8.56 & 179 & 8.60 & 191 & 8.30 & 62 & 8.12 & 82 & 8.24 \\
\hline O II & 4416.98 & 153 & 8.55 & 136 & 8.58 & 144 & 8.32 & 46 & 8.14 & 68 & 8.36 \\
\hline O II & 4443.01 & - & - & - & - & - & - & - & - & - & - \\
\hline O II & 4452.38 & 61 & 8.62 & 56 & 8.64 & - & - & 21 & 8.28 & 28 & 8.36 \\
\hline O II & 4590.97 & 140 & 8.49 & 126 & 8.53 & 71 & 7.80 & 47 & 8.19 & 60 & 8.23 \\
\hline O II & 4596.18 & 116 & 8.45 & 109 & 8.51 & - & - & 39 & 8.10 & 65 & 8.36 \\
\hline O II & 4638.86 & 163 & 8.70 & 145 & 8.74 & - & - & 49 & 8.28 & 68 & 8.43 \\
\hline O II & 4641.81 & - & - & 212 & 8.68 & - & - & 70 & 8.22 & 88 & 8.28 \\
\hline O II & 4650.00 & - & - & - & - & - & - & - & - & - & - \\
\hline O II & 4661.63 & 171 & 8.67 & 148 & 8.67 & 141 & 8.31 & 48 & 8.20 & 65 & 8.31 \\
\hline O II & 4673.73 & 41 & 8.58 & 44 & 8.69 & - & - & 16 & 8.34 & 22 & 8.42 \\
\hline O II & 4676.23 & 149 & 8.67 & 130 & 8.69 & - & - & 42 & 8.20 & 54 & 8.25 \\
\hline O II & 4699.00 & 80 & 8.21 & 84 & 8.31 & - & - & 38 & 8.00 & 51 & 8.07 \\
\hline O II & 4705.35 & 95 & 8.32 & 92 & 8.39 & - & - & 33 & 7.97 & 44 & 8.05 \\
\hline O II & 4710.00 & 28 & 8.48 & 43 & 8.80 & - & - & - & - & 19 & 8.44 \\
\hline Mg II & 4481.00 & 144 & 7.07 & 116 & 6.94 & 148 & 7.22 & 100 & 7.08 & 93 & 7.03 \\
\hline Si II & 4128.05 & - & - & - & - & - & - & 24 & 7.43 & - & - \\
\hline Si II & 4130.89 & - & - & - & - & - & - & 24 & 7.18 & - & - \\
\hline Si III & 4552.62 & 318 & 7.14 & 270 & 7.19 & 360 & 7.11 & 114 & 7.26 & 129 & 7.22 \\
\hline Si III & 4567.84 & 266 & 7.18 & 231 & 7.25 & 308 & 7.18 & 95 & 7.27 & 106 & 7.19 \\
\hline Si III & 4574.75 & 163 & 7.22 & 142 & 7.25 & 184 & 7.22 & 60 & 7.23 & 69 & 7.21 \\
\hline Si IV & 4088.90 & - & - & - & - & - & - & - & - & - & - \\
\hline Si IV & 4116.10 & 63 & 7.23 & 52 & 7.22 & - & - & - & - & 28 & 7.20 \\
\hline Si IV & 4212.00 & - & - & - & - & - & - & - & - & - & - \\
\hline
\end{tabular}


I. Hunter et al.: Chemical compositions of B-type stars, Online Material p 48

Table 1. continued.

\begin{tabular}{|c|c|c|c|c|c|c|c|c|c|c|c|}
\hline \multirow[t]{2}{*}{ Species } & \multirow[t]{2}{*}{ Wavelength } & \multicolumn{2}{|c|}{ NGC 2004-030 } & \multicolumn{2}{|c|}{ NGC 2004-031 } & \multicolumn{2}{|c|}{ NGC 2004-032 } & \multicolumn{2}{|c|}{ NGC 2004-036 } & \multicolumn{2}{|c|}{ NGC 2004-041 } \\
\hline & & $E W$ & Abund & $E W$ & Abund & $E W$ & Abund & $E W$ & Abund & $E W$ & Abund \\
\hline C II & 4267.00 & 87 & 7.92 & 159 & 7.81 & 149 & 7.73 & 131 & 7.80 & 156 & 7.67 \\
\hline N II & 3955.85 & - & - & - & - & - & - & 22 & 7.46 & - & - \\
\hline N II & 3995.00 & - & - & 41 & 6.84 & 41 & 6.84 & 88 & 7.28 & 32 & 6.80 \\
\hline N II & 4447.03 & 30 & 7.43 & - & - & - & - & 41 & 7.30 & - & - \\
\hline N II & 4601.48 & - & - & - & - & - & - & - & - & - & - \\
\hline N II & 4613.86 & - & - & - & - & - & - & 30 & 7.51 & - & - \\
\hline N II & 4630.54 & 39 & 7.43 & 25 & 6.84 & 33 & 6.96 & 65 & 7.27 & 27 & 6.97 \\
\hline N II & 4643.08 & - & - & - & - & - & - & 42 & 7.46 & - & - \\
\hline O II & 3912.00 & 65 & 8.28 & 37 & 8.10 & 55 & 8.41 & 88 & 8.49 & - & - \\
\hline O II & 3919.30 & - & - & - & - & - & - & - & - & - & - \\
\hline O II & 3945.04 & 45 & 8.41 & 26 & 8.15 & 40 & 8.46 & 54 & 8.41 & - & - \\
\hline O II & 3954.36 & 73 & 8.37 & 32 & 7.96 & 46 & 8.23 & 80 & 8.37 & 34 & 8.28 \\
\hline O II & 3982.71 & 42 & 8.36 & 33 & 8.34 & - & - & 53 & 8.41 & - & - \\
\hline O II & 4069.00 & 213 & 8.71 & 100 & 8.27 & 110 & 8.37 & 174 & 8.51 & 70 & 8.27 \\
\hline O II & 4072.15 & 129 & 8.42 & 72 & 8.30 & 70 & 8.25 & 130 & 8.54 & 47 & 8.23 \\
\hline O II & 4075.86 & 121 & 8.15 & 84 & 8.30 & 90 & 8.36 & 133 & 8.37 & 62 & 8.36 \\
\hline O II & 4078.84 & - & - & 19 & 8.07 & - & - & 45 & 8.42 & - & - \\
\hline O II & 4132.80 & - & - & 30 & 8.19 & - & - & 49 & 8.28 & - & - \\
\hline O II & 4156.53 & - & - & - & - & - & - & 25 & 8.62 & - & - \\
\hline O II & 4185.44 & - & - & - & - & - & - & - & - & - & - \\
\hline O II & 4317.00 & 92 & 8.71 & 42 & 8.15 & 58 & 8.44 & - & - & 42 & 8.46 \\
\hline O II & 4319.63 & 101 & 8.79 & 47 & 8.24 & 63 & 8.52 & 106 & 8.69 & 38 & 8.36 \\
\hline O II & 4325.76 & - & - & - & - & - & - & 33 & 8.54 & - & - \\
\hline O II & 4349.43 & - & - & 48 & 7.94 & - & - & - & - & - & - \\
\hline O II & 4351.00 & - & - & - & - & - & - & 103 & 8.45 & - & - \\
\hline O II & 4353.58 & - & - & - & - & - & - & - & - & - & - \\
\hline O II & 4366.00 & 93 & 8.63 & 49 & 8.20 & 50 & 8.22 & 101 & 8.54 & 37 & 8.28 \\
\hline O II & 4369.27 & - & - & - & - & - & - & 24 & 8.32 & - & - \\
\hline O II & 4395.94 & - & - & - & - & - & - & - & - & - & - \\
\hline O II & 4414.90 & 146 & 8.55 & 73 & 8.17 & - & - & 157 & 8.59 & - & - \\
\hline O II & 4416.98 & 97 & 8.45 & 56 & 8.22 & - & - & 127 & 8.63 & - & - \\
\hline O II & 4443.01 & - & - & - & - & - & - & - & - & - & - \\
\hline O II & 4452.38 & - & - & - & - & - & - & 53 & 8.63 & - & - \\
\hline O II & 4590.97 & 96 & 8.30 & 46 & 8.10 & 60 & 8.34 & 109 & 8.48 & 41 & 8.37 \\
\hline O II & 4596.18 & 78 & 8.25 & 44 & 8.15 & 46 & 8.20 & 95 & 8.43 & 44 & 8.49 \\
\hline O II & 4638.86 & 103 & 8.61 & 60 & 8.37 & 59 & 8.36 & 114 & 8.60 & 50 & 8.57 \\
\hline O II & 4641.81 & 158 & 8.57 & 83 & 8.25 & 90 & 8.32 & 162 & 8.53 & 67 & 8.43 \\
\hline O II & 4650.00 & - & - & 173 & 8.35 & 118 & 7.98 & - & - & 118 & 8.34 \\
\hline O II & 4661.63 & 90 & 8.44 & 59 & 8.28 & - & - & 115 & 8.53 & - & - \\
\hline O II & 4673.73 & 37 & 8.78 & - & - & - & - & 36 & 8.58 & - & - \\
\hline O II & 4676.23 & 84 & 8.51 & 51 & 8.29 & - & - & 92 & 8.45 & - & - \\
\hline O II & 4699.00 & 65 & 8.12 & 44 & 8.10 & - & - & 75 & 8.21 & - & - \\
\hline O II & 4705.35 & 58 & 8.05 & 37 & 8.05 & - & - & 74 & 8.25 & 21 & 7.94 \\
\hline O II & 4710.00 & - & - & - & - & - & - & 34 & 8.65 & - & - \\
\hline Mg II & 4481.00 & 83 & 7.16 & 138 & 7.17 & 129 & 7.07 & 96 & 6.93 & 121 & 7.01 \\
\hline Si II & 4128.05 & - & - & 25 & 7.15 & - & - & - & - & - & - \\
\hline Si II & 4130.89 & - & - & 41 & 7.31 & - & - & - & - & - & - \\
\hline Si III & 4552.62 & 160 & 7.10 & 145 & 7.19 & 145 & 7.13 & 205 & 7.14 & 104 & 7.17 \\
\hline Si III & 4567.84 & 141 & 7.24 & 120 & 7.19 & 119 & 7.13 & 180 & 7.23 & 81 & 7.11 \\
\hline Si III & 4574.75 & 71 & 7.20 & 70 & 7.11 & 78 & 7.19 & 115 & 7.25 & 60 & 7.31 \\
\hline Si IV & 4088.90 & - & - & - & - & - & - & - & - & - & - \\
\hline Si IV & 4116.10 & - & - & - & - & - & - & 46 & 7.18 & - & - \\
\hline Si IV & 4212.00 & - & - & - & - & - & - & - & - & - & - \\
\hline
\end{tabular}


I. Hunter et al.: Chemical compositions of B-type stars, Online Material p 49

Table 1. continued.

\begin{tabular}{|c|c|c|c|c|c|c|c|c|c|c|c|}
\hline \multirow[t]{2}{*}{ Species } & \multirow[t]{2}{*}{ Wavelength } & \multicolumn{2}{|c|}{ NGC 2004-042 } & \multicolumn{2}{|c|}{ NGC 2004-043 } & \multicolumn{2}{|c|}{ NGC 2004-045 } & \multicolumn{2}{|c|}{ NGC 2004-046 } & \multicolumn{2}{|c|}{ NGC 2004-047 } \\
\hline & & $E W$ & Abund & $E W$ & Abund & $E W$ & Abund & $E W$ & Abund & $E W$ & Abund \\
\hline C II & 4267.00 & 165 & 7.80 & 131 & 7.78 & 119 & 7.58 & 95 & 7.80 & 120 & 7.56 \\
\hline N II & 3955.85 & - & - & 16 & 7.31 & - & - & 13 & 7.44 & - & - \\
\hline N II & 3995.00 & 37 & 6.88 & 73 & 7.14 & 38 & 6.89 & 81 & 7.54 & 46 & 6.91 \\
\hline N II & 4447.03 & - & - & 31 & 7.14 & _- & - & 42 & 7.49 & - & - \\
\hline N II & 4601.48 & - & - & - & - & - & - & - & - & - & - \\
\hline N II & 4613.86 & - & - & 37 & 7.65 & - & - & 33 & 7.86 & - & - \\
\hline N II & 4630.54 & 25 & 6.92 & 52 & 7.15 & 20 & 6.79 & 68 & 7.64 & 47 & 7.16 \\
\hline N II & 4643.08 & - & - & 34 & 7.36 & - & - & 34 & 7.61 & - & - \\
\hline O II & 3912.00 & 34 & 8.28 & 86 & 8.54 & 45 & 8.43 & 68 & 8.35 & 47 & 8.27 \\
\hline O II & 3919.30 & - & - & - & - & - & - & - & - & - & - \\
\hline $\mathrm{O}$ II & 3945.04 & 17 & 8.09 & 60 & 8.55 & 35 & 8.56 & 48 & 8.43 & 38 & 8.42 \\
\hline O II & 3954.36 & 26 & 8.06 & 70 & 8.34 & 47 & 8.51 & 60 & 8.27 & 38 & 8.08 \\
\hline O II & 3982.71 & - & - & 55 & 8.50 & - & - & 44 & 8.39 & - & - \\
\hline O II & 4069.00 & 58 & 8.07 & 168 & 8.54 & - & - & 144 & 8.41 & 98 & 8.25 \\
\hline O II & 4072.15 & 36 & 7.93 & 102 & 8.31 & - & - & 93 & 8.35 & 96 & 8.67 \\
\hline O II & 4075.86 & 55 & 8.18 & 118 & 8.30 & - & - & 98 & 8.22 & 85 & 8.31 \\
\hline O II & 4078.84 & 12 & 8.00 & 47 & 8.51 & - & - & 33 & 8.26 & - & - \\
\hline O II & 4132.80 & - & - & 52 & 8.38 & - & - & 54 & 8.43 & - & - \\
\hline O II & 4156.53 & - & - & 20 & 8.52 & - & - & 16 & 8.34 & - & - \\
\hline O II & 4185.44 & - & - & - & - & - & - & - & - & - & - \\
\hline O II & 4317.00 & 34 & 8.24 & 100 & 8.70 & - & - & 75 & 8.54 & 53 & 8.35 \\
\hline O II & 4319.63 & 28 & 8.08 & 100 & 8.70 & - & - & 74 & 8.52 & 52 & 8.33 \\
\hline O II & 4325.76 & - & - & 32 & 8.58 & - & - & 27 & 8.52 & - & - \\
\hline O II & 4349.43 & - & - & 131 & 8.64 & - & - & 120 & 8.83 & - & - \\
\hline O II & 4351.00 & - & - & 91 & 8.44 & - & - & 79 & 8.26 & - & - \\
\hline O II & 4353.58 & - & - & - & - & - & - & - & - & - & - \\
\hline O II & 4366.00 & 33 & 8.15 & 93 & 8.52 & - & - & 74 & 8.44 & - & - \\
\hline O II & 4369.27 & - & - & 25 & 8.40 & - & - & - & - & - & - \\
\hline O II & 4395.94 & - & - & 59 & 8.77 & - & - & 54 & 8.73 & - & - \\
\hline O II & 4414.90 & 47 & 8.06 & 136 & 8.50 & 68 & 8.39 & 111 & 8.39 & 79 & 8.25 \\
\hline O II & 4416.98 & 38 & 8.18 & 115 & 8.60 & 44 & 8.26 & 100 & 8.58 & 53 & 8.17 \\
\hline O II & 4443.01 & - & - & - & - & - & - & - & - & - & - \\
\hline O II & 4452.38 & 23 & 8.55 & 53 & 8.69 & - & - & 48 & 8.65 & - & - \\
\hline O II & 4590.97 & - & - & 102 & 8.51 & 35 & 8.11 & 91 & 8.46 & 70 & 8.48 \\
\hline O II & 4596.18 & - & - & 95 & 8.52 & - & - & 87 & 8.45 & 70 & 8.54 \\
\hline O II & 4638.86 & 42 & 8.38 & 110 & 8.65 & 47 & 8.42 & 86 & 8.50 & 63 & 8.42 \\
\hline O II & 4641.81 & 52 & 8.14 & 160 & 8.61 & 63 & 8.30 & 118 & 8.43 & 96 & 8.41 \\
\hline O II & 4650.00 & - & - & - & - & 172 & 8.73 & - & - & 207 & 8.56 \\
\hline O II & 4661.63 & 36 & 8.18 & 108 & 8.55 & 54 & 8.50 & 85 & 8.42 & - & - \\
\hline O II & 4673.73 & - & - & 39 & 8.70 & - & - & 37 & 8.69 & 25 & 8.59 \\
\hline O II & 4676.23 & - & - & 93 & 8.54 & - & - & 68 & 8.32 & 70 & 8.59 \\
\hline O II & 4699.00 & - & - & 91 & 8.43 & - & - & 77 & 8.19 & 55 & 8.26 \\
\hline O II & 4705.35 & - & - & 82 & 8.41 & - & - & 61 & 8.12 & 46 & 8.21 \\
\hline O II & 4710.00 & - & - & 33 & 8.70 & - & - & 38 & 8.76 & - & - \\
\hline $\mathrm{Mg}$ II & 4481.00 & 134 & 7.14 & 102 & 6.95 & 98 & 6.97 & 76 & 7.02 & 101 & 6.90 \\
\hline Si II & 4128.05 & 27 & 7.16 & 24 & 7.24 & - & - & - & - & - & - \\
\hline Si II & 4130.89 & 41 & 7.32 & 22 & 6.97 & - & - & - & - & - & - \\
\hline Si III & 4552.62 & 111 & 7.24 & 190 & 7.10 & 108 & 7.31 & 129 & 7.13 & 140 & 7.14 \\
\hline Si III & 4567.84 & 83 & 7.12 & 165 & 7.18 & 80 & 7.16 & 113 & 7.21 & 121 & 7.20 \\
\hline Si III & 4574.75 & 55 & 7.20 & 111 & 7.27 & 42 & 6.97 & 79 & 7.33 & 74 & 7.17 \\
\hline Si IV & 4088.90 & - & - & - & - & - & - & - & - & - & - \\
\hline Si IV & 4116.10 & - & - & 35 & 7.14 & - & - & 54 & 7.22 & - & - \\
\hline Si IV & 4212.00 & - & - & - & - & - & - & - & - & - & - \\
\hline
\end{tabular}


I. Hunter et al.: Chemical compositions of B-type stars, Online Material p 50

Table 1. continued.

\begin{tabular}{|c|c|c|c|c|c|c|c|c|c|c|c|}
\hline \multirow[t]{2}{*}{ Species } & \multirow[t]{2}{*}{ Wavelength } & \multicolumn{2}{|c|}{ NGC 2004-050 } & \multicolumn{2}{|c|}{ NGC 2004-051 } & \multicolumn{2}{|c|}{ NGC 2004-052 } & \multicolumn{2}{|c|}{ NGC 2004-053 } & \multicolumn{2}{|c|}{ NGC 2004-054 } \\
\hline & & $E W$ & Abund & $E W$ & Abund & $E W$ & Abund & $E W$ & Abund & $E W$ & Abund \\
\hline C II & 4267.00 & 141 & 7.57 & 150 & 7.73 & 134 & 7.59 & 76 & 7.99 & 147 & 7.78 \\
\hline N II & 3955.85 & - & - & - & - & - & - & - & - & - & - \\
\hline N II & 3995.00 & 50 & 7.11 & 42 & 6.85 & 42 & 6.92 & 50 & 7.63 & 46 & 6.98 \\
\hline N II & 4447.03 & - & - & - & - & - & - & - & - & - & - \\
\hline N II & 4601.48 & - & - & - & - & - & - & - & - & - & - \\
\hline N II & 4613.86 & - & - & - & - & - & - & - & - & - & - \\
\hline N II & 4630.54 & 33 & 7.08 & 33 & 6.96 & 21 & 6.84 & 35 & 7.61 & 30 & 6.95 \\
\hline N II & 4643.08 & - & - & 20 & 7.12 & - & - & 16 & 7.67 & - & - \\
\hline O II & 3912.00 & 39 & 8.41 & 67 & 8.57 & 38 & 8.29 & 50 & 8.29 & 50 & 8.43 \\
\hline O II & 3919.30 & - & - & - & - & - & - & - & - & - & - \\
\hline O II & 3945.04 & 29 & 8.48 & 38 & 8.42 & 41 & 8.65 & 31 & 8.39 & 32 & 8.39 \\
\hline O II & 3954.36 & 30 & 8.18 & 61 & 8.47 & 51 & 8.51 & 49 & 8.32 & 50 & 8.44 \\
\hline O II & 3982.71 & - & - & 36 & 8.41 & - & - & 29 & 8.36 & - & - \\
\hline O II & 4069.00 & 77 & 8.37 & 142 & 8.67 & 132 & 8.78 & 145 & 8.49 & 110 & 8.56 \\
\hline O II & 4072.15 & 42 & 8.11 & 86 & 8.49 & 65 & 8.38 & 66 & 8.04 & 75 & 8.59 \\
\hline O II & 4075.86 & 70 & 8.51 & 113 & 8.67 & 86 & 8.51 & 82 & 8.04 & 80 & 8.49 \\
\hline O II & 4078.84 & - & - & 32 & 8.45 & - & - & 40 & 8.57 & - & - \\
\hline O II & 4132.80 & - & - & 38 & 8.38 & - & - & - & - & - & - \\
\hline O II & 4156.53 & - & - & - & - & - & - & - & - & - & - \\
\hline O II & 4185.44 & - & - & - & - & - & - & - & - & - & - \\
\hline O II & 4317.00 & 43 & 8.48 & 89 & 8.90 & 54 & 8.56 & 55 & 8.57 & 66 & 8.76 \\
\hline O II & 4319.63 & 48 & 8.59 & 84 & 8.83 & 67 & 8.78 & 60 & 8.62 & 48 & 8.40 \\
\hline O II & 4325.76 & - & - & 20 & 8.45 & - & - & - & - & - & - \\
\hline O II & 4349.43 & - & - & - & - & - & - & - & - & - & - \\
\hline O II & 4351.00 & - & - & - & - & - & - & 55 & 8.01 & - & - \\
\hline O II & 4353.58 & - & - & - & - & - & - & 12 & 8.16 & - & - \\
\hline O II & 4366.00 & - & - & 64 & 8.45 & 65 & 8.66 & 58 & 8.53 & 47 & 8.32 \\
\hline O II & 4369.27 & - & - & - & - & - & - & 12 & 8.17 & - & - \\
\hline O II & 4395.94 & - & - & 34 & 8.61 & - & - & 30 & 8.45 & - & - \\
\hline O II & 4414.90 & - & - & 80 & 8.26 & 107 & 8.77 & 79 & 8.35 & 73 & 8.35 \\
\hline O II & 4416.98 & - & - & 62 & 8.31 & 46 & 8.22 & 69 & 8.48 & 58 & 8.42 \\
\hline O II & 4443.01 & - & - & - & - & - & - & - & - & - & - \\
\hline O II & 4452.38 & - & - & 35 & 8.61 & - & - & 25 & 8.56 & - & - \\
\hline O II & 4590.97 & 40 & 8.34 & 73 & 8.53 & 64 & 8.63 & 69 & 8.31 & 53 & 8.38 \\
\hline O II & 4596.18 & 47 & 8.55 & 81 & 8.68 & 56 & 8.57 & 59 & 8.30 & 66 & 8.63 \\
\hline O II & 4638.86 & 43 & 8.43 & 84 & 8.71 & 54 & 8.49 & 73 & 8.61 & - & - \\
\hline O II & 4641.81 & 64 & 8.38 & 125 & 8.71 & 87 & 8.51 & 107 & 8.52 & - & - \\
\hline O II & 4650.00 & 127 & 8.42 & - & - & 197 & 8.71 & - & - & - & - \\
\hline O II & 4661.63 & 43 & 8.36 & 77 & 8.54 & 67 & 8.62 & 72 & 8.53 & 61 & 8.49 \\
\hline O II & 4673.73 & - & - & - & - & - & - & 30 & 8.89 & - & - \\
\hline O II & 4676.23 & - & - & - & - & - & - & 49 & 8.41 & - & - \\
\hline O II & 4699.00 & - & - & 52 & 8.24 & 58 & 8.53 & 61 & 8.23 & 37 & 8.06 \\
\hline O II & 4705.35 & 32 & 8.26 & 43 & 8.17 & 43 & 8.37 & 57 & 8.20 & 38 & 8.16 \\
\hline O II & 4710.00 & - & - & 24 & 8.73 & - & - & 26 & 8.60 & - & - \\
\hline Mg II & 4481.00 & 124 & 7.03 & 118 & 6.99 & 113 & 6.90 & 80 & 7.24 & 124 & 7.19 \\
\hline Si II & 4128.05 & - & - & 25 & 7.10 & - & - & - & - & - & - \\
\hline Si II & 4130.89 & - & - & 25 & 6.87 & - & - & - & - & - & - \\
\hline Si III & 4552.62 & 101 & 7.13 & 142 & 7.10 & 124 & 7.09 & 103 & 7.09 & 116 & 7.23 \\
\hline Si III & 4567.84 & 93 & 7.29 & 132 & 7.27 & 105 & 7.14 & 89 & 7.21 & 96 & 7.23 \\
\hline Si III & 4574.75 & 60 & 7.31 & 87 & 7.31 & 79 & 7.36 & 45 & 7.23 & 62 & 7.24 \\
\hline Si IV & 4088.90 & - & - & - & - & - & - & - & - & - & - \\
\hline Si IV & 4116.10 & - & - & 30 & 7.48 & - & - & 133 & 7.18 & - & - \\
\hline Si IV & 4212.00 & - & - & - & - & - & - & - & - & - & - \\
\hline
\end{tabular}


I. Hunter et al.: Chemical compositions of B-type stars, Online Material p 51

Table 1. continued.

\begin{tabular}{|c|c|c|c|c|c|c|c|c|c|c|c|}
\hline \multirow[t]{2}{*}{ Species } & \multirow[t]{2}{*}{ Wavelength } & \multicolumn{2}{|c|}{ NGC 2004-059 } & \multicolumn{2}{|c|}{ NGC 2004-060 } & \multicolumn{2}{|c|}{ NGC 2004-061 } & \multicolumn{2}{|c|}{ NGC 2004-062 } & \multicolumn{2}{|c|}{ NGC 2004-063 } \\
\hline & & $E W$ & Abund & $E W$ & Abund & $E W$ & Abund & $E W$ & Abund & $E W$ & Abund \\
\hline C II & 4267.00 & 154 & 7.82 & 136 & 7.62 & 175 & 7.91 & 66 & 7.89 & 138 & 7.72 \\
\hline N II & 3955.85 & - & - & - & - & - & - & - & - & - & - \\
\hline N II & 3995.00 & 46 & 6.99 & 32 & 6.75 & 41 & 6.94 & $<30$ & $<7.33$ & 43 & 6.98 \\
\hline N II & 4447.03 & - & - & - & - & 18 & 6.99 & - & - & - & - \\
\hline N II & 4601.48 & - & - & - & - & - & - & - & - & - & - \\
\hline N II & 4613.86 & - & - & - & - & - & - & - & - & - & - \\
\hline N II & 4630.54 & 32 & 7.00 & 35 & 7.06 & 33 & 7.05 & - & - & 28 & 6.95 \\
\hline N II & 4643.08 & - & - & - & - & - & - & - & - & - & - \\
\hline O II & 3912.00 & - & - & 69 & 8.77 & 28 & 8.09 & - & - & 50 & 8.52 \\
\hline O II & 3919.30 & - & - & - & - & - & - & - & - & - & - \\
\hline O II & 3945.04 & 43 & 8.68 & 30 & 8.40 & - & - & - & - & - & - \\
\hline O II & 3954.36 & 46 & 8.40 & 45 & 8.40 & 36 & 8.26 & 35 & 8.17 & 38 & 8.30 \\
\hline O II & 3982.71 & - & - & - & - & 18 & 8.12 & - & - & - & - \\
\hline O II & 4069.00 & 101 & 8.49 & - & - & 71 & 8.21 & - & - & 114 & 8.73 \\
\hline O II & 4072.15 & 70 & 8.53 & - & - & 54 & 8.32 & - & - & 83 & 8.90 \\
\hline O II & 4075.86 & 96 & 8.79 & - & - & 65 & 8.37 & - & - & 86 & 8.75 \\
\hline O II & 4078.84 & 29 & 8.52 & - & - & 18 & 8.22 & - & - & 41 & 8.94 \\
\hline O II & 4132.80 & - & - & - & - & - & - & - & - & - & - \\
\hline O II & 4156.53 & - & - & - & - & - & - & - & - & - & - \\
\hline O II & 4185.44 & - & - & - & - & - & - & - & - & - & - \\
\hline O II & 4317.00 & 54 & 8.56 & 48 & 8.44 & 32 & 8.14 & - & - & - & - \\
\hline O II & 4319.63 & 49 & 8.46 & 48 & 8.44 & 39 & 8.32 & - & - & - & - \\
\hline O II & 4325.76 & - & - & - & - & - & - & - & - & - & - \\
\hline O II & 4349.43 & - & - & - & - & - & - & - & - & - & - \\
\hline O II & 4351.00 & - & - & - & - & - & - & - & - & - & - \\
\hline O II & 4353.58 & - & - & - & - & - & - & - & - & - & - \\
\hline O II & 4366.00 & 37 & 8.13 & 52 & 8.45 & 30 & 8.04 & - & - & 42 & 8.33 \\
\hline O II & 4369.27 & - & - & - & - & - & - & - & - & - & - \\
\hline O II & 4395.94 & - & - & - & - & - & - & - & - & - & - \\
\hline O II & 4414.90 & - & - & - & - & 63 & 8.32 & - & - & - & - \\
\hline O II & 4416.98 & - & - & - & - & 53 & 8.46 & - & - & - & - \\
\hline O II & 4443.01 & - & - & - & - & - & - & - & - & - & - \\
\hline O II & 4452.38 & - & - & - & - & 37 & 8.90 & - & - & - & - \\
\hline O II & 4590.97 & 53 & 8.42 & 47 & 8.34 & - & - & - & - & 48 & 8.41 \\
\hline O II & 4596.18 & 48 & 8.38 & 66 & 8.70 & - & - & - & - & 42 & 8.34 \\
\hline O II & 4638.86 & 50 & 8.40 & 77 & 8.86 & 46 & 8.42 & - & - & 48 & 8.44 \\
\hline O II & 4641.81 & 74 & 8.38 & 84 & 8.50 & 66 & 8.35 & - & - & 81 & 8.60 \\
\hline O II & 4650.00 & 147 & 8.42 & 171 & 8.57 & - & - & - & - & 133 & 8.40 \\
\hline O II & 4661.63 & - & - & - & - & 44 & 8.31 & 26 & 7.91 & - & - \\
\hline O II & 4673.73 & - & - & - & - & - & - & - & - & - & - \\
\hline O II & 4676.23 & 43 & 8.31 & - & - & - & - & - & - & - & - \\
\hline O II & 4699.00 & 39 & 8.13 & 58 & 8.49 & - & - & - & - & - & - \\
\hline O II & 4705.35 & 45 & 8.35 & - & - & - & - & - & - & - & - \\
\hline O II & 4710.00 & - & - & - & - & - & - & - & - & - & - \\
\hline Mg II & 4481.00 & 112 & 7.07 & 119 & 7.02 & 125 & 7.13 & 40 & 6.83 & 104 & 7.03 \\
\hline Si II & 4128.05 & - & - & - & - & 33 & 7.45 & - & - & - & - \\
\hline Si II & 4130.89 & - & - & - & - & 28 & 7.01 & - & - & - & - \\
\hline Si III & 4552.62 & 114 & 7.24 & 121 & 7.17 & 99 & 7.12 & 81 & 7.12 & 91 & 7.07 \\
\hline Si III & 4567.84 & 96 & 7.26 & 109 & 7.30 & 82 & 7.13 & 71 & 7.24 & 86 & 7.26 \\
\hline Si III & 4574.75 & 55 & 7.14 & 66 & 7.24 & 58 & 7.27 & 41 & 7.28 & 51 & 7.17 \\
\hline Si IV & 4088.90 & - & - & - & - & - & - & - & - & - & - \\
\hline Si IV & 4116.10 & - & - & - & - & - & - & 88 & 7.21 & - & - \\
\hline Si IV & 4212.00 & - & - & - & - & - & - & - & - & - & - \\
\hline
\end{tabular}


I. Hunter et al.: Chemical compositions of B-type stars, Online Material p 52

Table 1. continued.

\begin{tabular}{|c|c|c|c|c|c|c|c|c|c|c|c|}
\hline \multirow[t]{2}{*}{ Species } & \multirow[t]{2}{*}{ Wavelength } & \multicolumn{2}{|c|}{ NGC 2004-064 } & \multicolumn{2}{|c|}{ NGC 2004-065 } & \multicolumn{2}{|c|}{ NGC 2004-066 } & \multicolumn{2}{|c|}{ NGC 2004-068 } & \multicolumn{2}{|c|}{ NGC 2004-069 } \\
\hline & & $E W$ & Abund & $E W$ & Abund & $E W$ & Abund & $E W$ & Abund & $E W$ & Abund \\
\hline C II & 4267.00 & 94 & 7.78 & 155 & 7.70 & 92 & 7.78 & 149 & 7.69 & 93 & 7.90 \\
\hline N II & 3955.85 & 17 & 7.55 & - & - & - & - & - & - & - & - \\
\hline N II & 3995.00 & 96 & 7.56 & $<50$ & $<7.19$ & 92 & 7.64 & 35 & 7.01 & 89 & 7.68 \\
\hline N II & 4447.03 & 46 & 7.48 & - & - & - & - & - & - & - & - \\
\hline N II & 4601.48 & 31 & 7.50 & - & - & - & - & - & - & - & - \\
\hline N II & 4613.86 & - & - & - & - & - & - & - & - & - & - \\
\hline N II & 4630.54 & - & - & - & - & 121 & 8.32 & - & - & 93 & 7.99 \\
\hline N II & 4643.08 & 34 & 7.54 & - & - & - & - & - & - & - & - \\
\hline O II & 3912.00 & 27 & 7.68 & - & - & 98 & 8.65 & 31 & 8.50 & 45 & 8.02 \\
\hline O II & 3919.30 & - & - & - & - & - & - & - & - & - & - \\
\hline O II & 3945.04 & 52 & 8.37 & - & - & - & - & - & - & - & - \\
\hline O II & 3954.36 & 68 & 8.22 & - & - & 54 & 8.15 & 27 & 8.40 & 48 & 8.06 \\
\hline O II & 3982.71 & 54 & 8.41 & - & - & - & - & - & - & - & - \\
\hline O II & 4069.00 & 169 & 8.38 & - & - & - & - & 54 & 8.33 & - & - \\
\hline O II & 4072.15 & 113 & 8.29 & - & - & - & - & 24 & 7.91 & - & - \\
\hline O II & 4075.86 & 112 & 8.08 & - & - & - & - & - & - & - & - \\
\hline O II & 4078.84 & 35 & 8.19 & - & - & - & - & - & - & - & - \\
\hline O II & 4132.80 & - & - & - & - & - & - & - & - & - & - \\
\hline O II & 4156.53 & - & - & - & - & - & - & - & - & - & - \\
\hline O II & 4185.44 & - & - & - & - & - & - & - & - & - & - \\
\hline O II & 4317.00 & 88 & 8.49 & - & - & 98 & 8.87 & - & - & - & - \\
\hline O II & 4319.63 & 103 & 8.66 & - & - & 68 & 8.39 & - & - & - & - \\
\hline O II & 4325.76 & 43 & 8.74 & - & - & - & - & - & - & - & - \\
\hline O II & 4349.43 & 137 & 8.62 & - & - & - & - & - & - & - & - \\
\hline O II & 4351.00 & 91 & 8.22 & - & - & - & - & - & - & - & - \\
\hline O II & 4353.58 & - & - & - & - & - & - & - & - & - & - \\
\hline O II & 4366.00 & 87 & 8.39 & - & - & 74 & 8.41 & 20 & 8.07 & - & - \\
\hline O II & 4369.27 & 14 & 8.01 & - & - & - & - & - & - & - & - \\
\hline O II & 4395.94 & 35 & 8.30 & - & - & - & - & - & - & - & - \\
\hline O II & 4414.90 & 128 & 8.33 & - & - & 150 & 8.75 & - & - & 135 & 8.49 \\
\hline O II & 4416.98 & 125 & 8.58 & - & - & 102 & 8.57 & - & - & 100 & 8.45 \\
\hline O II & 4443.01 & - & - & - & - & - & - & - & - & - & - \\
\hline O II & 4452.38 & 49 & 8.55 & - & - & - & - & - & - & - & - \\
\hline O II & 4590.97 & 112 & 8.43 & - & - & 96 & 8.48 & - & - & 77 & 8.14 \\
\hline O II & 4596.18 & 91 & 8.31 & - & - & 69 & 8.19 & - & - & 55 & 7.99 \\
\hline O II & 4638.86 & 107 & 8.51 & - & - & - & - & 31 & 8.46 & - & - \\
\hline O II & 4641.81 & 154 & 8.45 & - & - & - & - & 41 & 8.28 & - & - \\
\hline O II & 4650.00 & - & - & - & - & - & - & - & - & - & - \\
\hline O II & 4661.63 & 105 & 8.41 & - & - & 87 & 8.41 & - & - & 75 & 8.20 \\
\hline O II & 4673.73 & 40 & 8.63 & - & - & - & - & - & - & - & - \\
\hline O II & 4676.23 & 87 & 8.38 & - & - & - & - & - & - & - & - \\
\hline O II & 4699.00 & 75 & 8.11 & - & - & - & - & - & - & - & - \\
\hline O II & 4705.35 & 74 & 8.15 & - & - & - & - & - & - & - & - \\
\hline O II & 4710.00 & 51 & 8.85 & - & - & - & - & - & - & - & - \\
\hline Mg II & 4481.00 & 90 & 7.08 & 158 & 7.34 & 64 & 6.88 & 135 & 7.11 & 89 & 7.17 \\
\hline Si II & 4128.05 & - & - & - & - & - & - & 44 & 7.78 & - & - \\
\hline Si II & 4130.89 & - & - & - & - & - & - & 50 & 7.66 & - & - \\
\hline Si III & 4552.62 & 174 & 7.10 & 90 & 7.15 & 140 & 7.21 & - & - & 155 & 7.23 \\
\hline Si III & 4567.84 & 152 & 7.19 & - & - & - & - & 64 & 7.16 & 119 & 7.14 \\
\hline Si III & 4574.75 & 97 & 7.24 & - & - & - & - & 45 & 7.31 & 73 & 7.19 \\
\hline Si IV & 4088.90 & - & - & - & - & - & - & - & - & - & - \\
\hline Si IV & 4116.10 & 75 & 7.18 & - & - & - & - & - & - & - & - \\
\hline Si IV & 4212.00 & - & - & - & - & - & - & - & - & - & - \\
\hline
\end{tabular}


I. Hunter et al.: Chemical compositions of B-type stars, Online Material p 53

Table 1. continued.

\begin{tabular}{|c|c|c|c|c|c|c|c|c|c|c|c|}
\hline \multirow[t]{2}{*}{ Species } & \multirow[t]{2}{*}{ Wavelength } & \multicolumn{2}{|c|}{ NGC 2004-070 } & \multicolumn{2}{|c|}{ NGC 2004-071 } & \multicolumn{2}{|c|}{ NGC 2004-073 } & \multicolumn{2}{|c|}{ NGC 2004-074 } & \multicolumn{2}{|c|}{ NGC 2004-075 } \\
\hline & & $E W$ & Abund & $E W$ & Abund & $E W$ & Abund & $E W$ & Abund & $E W$ & Abund \\
\hline C II & 4267.00 & 88 & 7.84 & 95 & 7.58 & 146 & 7.93 & 87 & 7.78 & 173 & 7.93 \\
\hline N II & 3955.85 & - & - & - & - & - & - & - & - & - & - \\
\hline N II & 3995.00 & 82 & 7.59 & 79 & 7.36 & 46 & 6.96 & - & - & 56 & 7.16 \\
\hline N II & 4447.03 & 34 & 7.42 & - & - & 20 & 6.98 & - & - & - & - \\
\hline N II & 4601.48 & - & - & - & - & - & - & - & - & - & - \\
\hline N II & 4613.86 & - & - & - & - & - & - & - & - & - & - \\
\hline N II & 4630.54 & 45 & 7.40 & 81 & 7.62 & 31 & 6.96 & $<48$ & $<7.42$ & 29 & 6.97 \\
\hline N II & 4643.08 & 17 & 7.33 & - & - & 22 & 7.19 & - & - & - & - \\
\hline O II & 3912.00 & - & - & 71 & 8.52 & 58 & 8.45 & - & - & 46 & 8.43 \\
\hline O II & 3919.30 & - & - & - & - & - & - & - & - & - & - \\
\hline O II & 3945.04 & 53 & 8.45 & 46 & 8.49 & 34 & 8.36 & 61 & 8.60 & - & - \\
\hline O II & 3954.36 & 62 & 8.22 & - & - & 53 & 8.40 & 81 & 8.53 & - & - \\
\hline O II & 3982.71 & 51 & 8.44 & - & - & 28 & 8.25 & - & - & - & - \\
\hline O II & 4069.00 & 180 & 8.55 & 155 & 8.72 & 99 & 8.29 & - & - & - & - \\
\hline O II & 4072.15 & 127 & 8.62 & 98 & 8.65 & 73 & 8.40 & - & - & - & - \\
\hline O II & 4075.86 & 133 & 8.48 & 100 & 8.48 & 81 & 8.35 & - & - & - & - \\
\hline O II & 4078.84 & 51 & 8.53 & 43 & 8.63 & 28 & 8.36 & - & - & - & - \\
\hline O II & 4132.80 & 54 & 8.33 & - & - & 37 & 8.37 & - & - & - & - \\
\hline O II & 4156.53 & - & - & - & - & - & - & - & - & - & - \\
\hline O II & 4185.44 & - & - & - & - & - & - & - & - & - & - \\
\hline O II & 4317.00 & 90 & 8.64 & 67 & 8.52 & 70 & 8.72 & - & - & - & - \\
\hline O II & 4319.63 & 103 & 8.81 & 67 & 8.52 & 66 & 8.65 & - & - & - & - \\
\hline O II & 4325.76 & 42 & 8.80 & - & - & 21 & 8.49 & - & - & - & - \\
\hline O II & 4349.43 & 117 & 8.59 & - & - & 70 & 8.40 & - & - & - & - \\
\hline O II & 4351.00 & 86 & 8.20 & - & - & 46 & 8.16 & - & - & - & - \\
\hline O II & 4353.58 & - & - & - & - & - & - & - & - & - & - \\
\hline O II & 4366.00 & 88 & 8.51 & - & - & 53 & 8.34 & - & - & 42 & 8.28 \\
\hline O II & 4369.27 & 25 & 8.31 & - & - & 16 & 8.27 & - & - & - & - \\
\hline O II & 4395.94 & 50 & 8.56 & - & - & - & - & - & - & - & - \\
\hline O II & 4414.90 & 131 & 8.46 & - & - & 84 & 8.38 & - & - & 70 & 8.35 \\
\hline O II & 4416.98 & 119 & 8.64 & - & - & 79 & 8.62 & - & - & - & - \\
\hline O II & 4443.01 & - & - & - & - & - & - & - & - & - & - \\
\hline O II & 4452.38 & 48 & 8.59 & - & - & 31 & 8.52 & - & - & - & - \\
\hline O II & 4590.97 & - & - & 87 & 8.62 & 58 & 8.33 & - & - & - & - \\
\hline O II & 4596.18 & - & - & 79 & 8.54 & 56 & 8.35 & - & - & - & - \\
\hline O II & 4638.86 & 119 & 8.75 & 74 & 8.50 & 60 & 8.42 & - & - & - & - \\
\hline O II & 4641.81 & 95 & 8.05 & 134 & 8.80 & 83 & 8.34 & - & - & - & - \\
\hline O II & 4650.00 & - & - & 271 & 8.89 & - & - & - & - & - & - \\
\hline O II & 4661.63 & - & - & 88 & 8.62 & 61 & 8.37 & 88 & 8.40 & 56 & 8.48 \\
\hline O II & 4673.73 & - & - & - & - & 17 & 8.35 & - & - & - & - \\
\hline O II & 4676.23 & - & - & - & - & 55 & 8.39 & - & - & - & - \\
\hline O II & 4699.00 & - & - & - & - & 54 & 8.21 & - & - & 37 & 8.15 \\
\hline O II & 4705.35 & - & - & 66 & 8.39 & 47 & 8.20 & - & - & - & - \\
\hline O II & 4710.00 & - & - & - & - & 16 & 8.44 & - & - & - & - \\
\hline Mg II & 4481.00 & 86 & 7.15 & 94 & 7.00 & 107 & 7.12 & 75 & 7.02 & 118 & 7.07 \\
\hline Si II & 4128.05 & - & - & - & - & 20 & 7.24 & - & - & - & - \\
\hline Si II & 4130.89 & - & - & - & - & 25 & 7.18 & - & - & - & - \\
\hline Si III & 4552.62 & 139 & 7.09 & 135 & 7.18 & 123 & 7.23 & 126 & 6.99 & 109 & 7.12 \\
\hline Si III & 4567.84 & 124 & 7.21 & 114 & 7.20 & 100 & 7.20 & 119 & 7.18 & 96 & 7.22 \\
\hline Si III & 4574.75 & 81 & 7.28 & 73 & 7.19 & 67 & 7.25 & 87 & 7.37 & 54 & 7.10 \\
\hline Si IV & 4088.90 & - & - & - & - & - & - & - & - & - & - \\
\hline Si IV & 4116.10 & 77 & 7.20 & - & - & - & - & - & - & - & - \\
\hline Si IV & 4212.00 & - & - & - & - & - & - & - & - & - & - \\
\hline
\end{tabular}


I. Hunter et al.: Chemical compositions of B-type stars, Online Material p 54

Table 1. continued.

\begin{tabular}{|c|c|c|c|c|c|c|c|c|c|c|c|}
\hline \multirow[t]{2}{*}{ Species } & \multirow[t]{2}{*}{ Wavelength } & \multicolumn{2}{|c|}{ NGC 2004-076 } & \multicolumn{2}{|c|}{ NGC 2004-077 } & \multicolumn{2}{|c|}{ NGC 2004-078 } & \multicolumn{2}{|c|}{ NGC 2004-079 } & \multicolumn{2}{|c|}{ NGC 2004-080 } \\
\hline & & $E W$ & Abund & $E W$ & Abund & $E W$ & Abund & $E W$ & Abund & $E W$ & Abund \\
\hline C II & 4267.00 & 150 & 7.70 & 92 & 7.98 & 125 & 7.61 & 133 & 7.65 & 144 & 7.64 \\
\hline N II & 3955.85 & - & - & - & - & - & - & - & - & - & - \\
\hline N II & 3995.00 & - & - & - & - & 30 & 6.74 & 100 & 7.77 & 44 & 7.11 \\
\hline N II & 4447.03 & - & - & - & - & - & - & - & - & - & - \\
\hline N II & 4601.48 & - & - & - & - & - & - & - & - & - & - \\
\hline N II & 4613.86 & - & - & - & - & - & - & - & - & - & - \\
\hline N II & 4630.54 & 18 & 6.91 & 52 & 7.65 & - & - & 54 & 7.42 & 28 & 7.06 \\
\hline N II & 4643.08 & 8 & 6.92 & - & - & - & - & - & - & - & - \\
\hline O II & 3912.00 & 30 & 8.47 & 73 & 8.39 & - & - & 38 & 8.31 & 34 & 8.42 \\
\hline O II & 3919.30 & - & - & - & - & - & - & - & - & - & - \\
\hline O II & 3945.04 & 20 & 8.47 & - & - & - & - & - & - & - & - \\
\hline O II & 3954.36 & 25 & 8.34 & 82 & 8.52 & 44 & 8.44 & 59 & 8.72 & 26 & 8.19 \\
\hline O II & 3982.71 & 17 & 8.45 & - & - & - & - & - & - & - & - \\
\hline O II & 4069.00 & 62 & 8.46 & - & - & - & - & - & - & 73 & 8.47 \\
\hline O II & 4072.15 & 44 & 8.48 & - & - & - & - & - & - & 65 & 8.83 \\
\hline O II & 4075.86 & 58 & 8.62 & - & - & - & - & - & - & 87 & 9.06 \\
\hline O II & 4078.84 & 14 & 8.38 & - & - & - & - & - & - & - & - \\
\hline O II & 4132.80 & - & - & - & - & - & - & - & - & 46 & 9.07 \\
\hline O II & 4156.53 & - & - & - & - & - & - & - & - & - & - \\
\hline O II & 4185.44 & - & - & - & - & - & - & - & - & - & - \\
\hline O II & 4317.00 & 35 & 8.59 & - & - & - & - & - & - & 29 & 8.25 \\
\hline O II & 4319.63 & 25 & 8.29 & - & - & - & - & - & - & 41 & 8.59 \\
\hline O II & 4325.76 & - & - & - & - & - & - & - & - & - & - \\
\hline O II & 4349.43 & 39 & 8.52 & - & - & - & - & - & - & - & - \\
\hline O II & 4351.00 & 18 & 8.19 & - & - & - & - & - & - & - & - \\
\hline O II & 4353.58 & - & - & - & - & - & - & - & - & - & - \\
\hline O II & 4366.00 & 32 & 8.46 & - & - & - & - & - & - & - & - \\
\hline O II & 4369.27 & - & - & - & - & - & - & - & - & - & - \\
\hline O II & 4395.94 & 16 & 8.64 & - & - & - & - & - & - & - & - \\
\hline O II & 4414.90 & - & - & 128 & 8.50 & - & - & - & - & 55 & 8.41 \\
\hline O II & 4416.98 & - & - & 108 & 8.60 & - & - & - & - & 59 & 8.82 \\
\hline O II & 4443.01 & - & - & - & - & - & - & - & - & - & - \\
\hline O II & 4452.38 & 15 & 8.51 & - & - & - & - & - & - & - & - \\
\hline O II & 4590.97 & 32 & 8.49 & - & - & 39 & 8.21 & - & - & 43 & 8.59 \\
\hline O II & 4596.18 & 30 & 8.50 & - & - & 39 & 8.29 & - & - & 34 & 8.42 \\
\hline O II & 4638.86 & 35 & 8.56 & - & - & 43 & 8.33 & 43 & 8.34 & 37 & 8.45 \\
\hline O II & 4641.81 & 47 & 8.41 & - & - & 56 & 8.15 & 91 & 8.68 & 45 & 8.21 \\
\hline O II & 4650.00 & - & - & - & - & 132 & 8.37 & 145 & 8.46 & 92 & 8.26 \\
\hline O II & 4661.63 & 27 & 8.28 & 75 & 8.32 & 49 & 8.39 & - & - & 34 & 8.31 \\
\hline O II & 4673.73 & - & - & - & - & - & - & - & - & - & - \\
\hline O II & 4676.23 & - & - & - & - & - & - & - & - & - & - \\
\hline O II & 4699.00 & 24 & 8.25 & - & - & - & - & - & - & - & - \\
\hline O II & 4705.35 & 29 & 8.47 & - & - & - & - & - & - & 30 & 8.34 \\
\hline O II & 4710.00 & - & - & - & - & - & - & - & - & - & - \\
\hline Mg II & 4481.00 & 122 & 7.01 & 74 & 7.10 & 100 & 6.95 & 115 & 7.03 & 117 & 7.05 \\
\hline Si II & 4128.05 & - & - & - & - & - & - & - & - & - & - \\
\hline Si II & 4130.89 & - & - & - & - & - & - & - & - & - & - \\
\hline Si III & 4552.62 & 87 & 7.27 & 151 & 7.24 & 95 & 7.04 & 99 & 7.02 & 88 & 7.24 \\
\hline Si III & 4567.84 & 69 & 7.25 & - & - & 85 & 7.16 & 87 & 7.12 & 78 & 7.34 \\
\hline Si III & 4574.75 & 40 & 7.19 & - & - & 59 & 7.27 & 64 & 7.31 & 41 & 7.13 \\
\hline Si IV & 4088.90 & - & - & - & - & - & - & - & - & - & - \\
\hline Si IV & 4116.10 & - & - & - & - & - & - & - & - & - & - \\
\hline Si IV & 4212.00 & - & - & - & - & - & - & - & - & - & - \\
\hline
\end{tabular}


I. Hunter et al.: Chemical compositions of B-type stars, Online Material p 55

Table 1. continued.

\begin{tabular}{|c|c|c|c|c|c|c|c|c|c|c|c|}
\hline \multirow[t]{2}{*}{ Species } & \multirow[t]{2}{*}{ Wavelength } & \multicolumn{2}{|c|}{ NGC 2004-081 } & \multicolumn{2}{|c|}{ NGC 2004-082 } & \multicolumn{2}{|c|}{ NGC 2004-084 } & \multicolumn{2}{|c|}{ NGC 2004-085 } & \multicolumn{2}{|c|}{ NGC 2004-086 } \\
\hline & & $E W$ & Abund & $E W$ & Abund & $E W$ & Abund & $E W$ & Abund & $E W$ & Abund \\
\hline C II & 4267.00 & 100 & 7.88 & 132 & 8.03 & 96 & 7.90 & 155 & 7.63 & 140 & 7.65 \\
\hline N II & 3955.85 & - & - & - & - & - & - & - & - & - & - \\
\hline N II & 3995.00 & 42 & 7.07 & - & - & 35 & 6.99 & 55 & 7.18 & 51 & 7.11 \\
\hline N II & 4447.03 & - & - & - & - & - & - & - & - & 26 & 7.27 \\
\hline N II & 4601.48 & - & - & - & - & - & - & - & - & - & - \\
\hline N II & 4613.86 & - & - & - & - & 18 & 7.57 & - & - & - & - \\
\hline N II & 4630.54 & 35 & 7.22 & 55 & 7.47 & 36 & 7.28 & 46 & 7.32 & 36 & 7.17 \\
\hline N II & 4643.08 & - & - & - & - & 15 & 7.26 & - & - & 20 & 7.30 \\
\hline O II & 3912.00 & 68 & 8.35 & 39 & 8.05 & 79 & 8.42 & - & - & 54 & 8.66 \\
\hline O II & 3919.30 & - & - & - & - & - & - & - & - & - & - \\
\hline O II & 3945.04 & 51 & 8.49 & - & - & 62 & 8.60 & - & - & 40 & 8.72 \\
\hline O II & 3954.36 & 67 & 8.38 & 54 & 8.33 & 75 & 8.42 & 38 & 8.39 & 54 & 8.66 \\
\hline O II & 3982.71 & - & - & - & - & 50 & 8.45 & - & - & 34 & 8.69 \\
\hline O II & 4069.00 & 136 & 8.33 & - & - & 168 & 8.51 & - & - & 108 & 8.64 \\
\hline O II & 4072.15 & 93 & 8.34 & - & - & 93 & 8.23 & - & - & 65 & 8.45 \\
\hline O II & 4075.86 & 104 & 8.30 & - & - & 109 & 8.25 & - & - & 87 & 8.58 \\
\hline O II & 4078.84 & 31 & 8.21 & - & - & 33 & 8.21 & - & - & 34 & 8.75 \\
\hline O II & 4132.80 & - & - & - & - & 54 & 8.36 & - & - & - & - \\
\hline O II & 4156.53 & - & - & - & - & 24 & 8.58 & - & - & - & - \\
\hline O II & 4185.44 & - & - & - & - & - & - & - & - & - & - \\
\hline O II & 4317.00 & 72 & 8.49 & 78 & 8.75 & 85 & 8.61 & 59 & 8.80 & 58 & 8.71 \\
\hline O II & 4319.63 & 61 & 8.31 & 46 & 8.16 & 79 & 8.52 & 35 & 8.30 & 62 & 8.78 \\
\hline O II & 4325.76 & - & - & - & - & 23 & 8.40 & - & - & - & - \\
\hline O II & 4349.43 & - & - & - & - & - & - & - & - & 79 & 8.83 \\
\hline O II & 4351.00 & - & - & - & - & 80 & 8.18 & - & - & 53 & 8.69 \\
\hline O II & 4353.58 & - & - & - & - & - & - & - & - & - & - \\
\hline O II & 4366.00 & 48 & 8.04 & - & - & 94 & 8.65 & - & - & 63 & 8.71 \\
\hline O II & 4369.27 & - & - & - & - & - & - & - & - & - & - \\
\hline O II & 4395.94 & - & - & - & - & 52 & 8.62 & - & - & 32 & 8.83 \\
\hline O II & 4414.90 & - & - & 49 & 7.74 & 120 & 8.40 & 51 & 8.16 & 80 & 8.51 \\
\hline O II & 4416.98 & - & - & 77 & 8.44 & 99 & 8.49 & 34 & 8.11 & 72 & 8.69 \\
\hline O II & 4443.01 & - & - & - & - & - & - & - & - & - & - \\
\hline O II & 4452.38 & - & - & - & - & 46 & 8.57 & - & - & 33 & 8.81 \\
\hline O II & 4590.97 & 82 & 8.34 & 73 & 8.40 & 98 & 8.45 & 48 & 8.53 & 55 & 8.58 \\
\hline O II & 4596.18 & 96 & 8.55 & 48 & 8.09 & 90 & 8.41 & - & - & 52 & 8.61 \\
\hline O II & 4638.86 & 74 & 8.35 & 71 & 8.47 & 102 & 8.62 & 58 & 8.71 & 59 & 8.66 \\
\hline O II & 4641.81 & 110 & 8.34 & 80 & 8.15 & 132 & 8.48 & 68 & 8.43 & 87 & 8.59 \\
\hline O II & 4650.00 & 255 & 8.62 & 214 & 8.57 & - & - & 133 & 8.46 & 170 & 8.62 \\
\hline O II & 4661.63 & 91 & 8.50 & 59 & 8.21 & - & - & 54 & 8.57 & 73 & 8.79 \\
\hline O II & 4673.73 & - & - & - & - & - & - & - & - & 29 & 9.00 \\
\hline O II & 4676.23 & - & - & - & - & 42 & 7.90 & - & - & 58 & 8.71 \\
\hline O II & 4699.00 & - & - & - & - & 84 & 8.20 & 25 & 8.06 & 48 & 8.48 \\
\hline O II & 4705.35 & - & - & - & - & 90 & 8.39 & - & - & 49 & 8.55 \\
\hline O II & 4710.00 & - & - & - & - & - & - & - & - & - & - \\
\hline Mg II & 4481.00 & 86 & 7.14 & 122 & 7.41 & 87 & 7.17 & 147 & 7.10 & 100 & 6.78 \\
\hline Si II & 4128.05 & - & - & - & - & - & - & - & - & - & - \\
\hline Si II & 4130.89 & - & - & - & - & - & - & - & - & - & - \\
\hline Si III & 4552.62 & 126 & 7.14 & 119 & 7.17 & 138 & 7.18 & 120 & 7.24 & 115 & 7.03 \\
\hline Si III & 4567.84 & 104 & 7.14 & 97 & 7.15 & 118 & 7.23 & 88 & 7.11 & 103 & 7.16 \\
\hline Si III & 4574.75 & 76 & 7.32 & - & - & 80 & 7.33 & 63 & 7.28 & 72 & 7.32 \\
\hline Si IV & 4088.90 & - & - & - & - & - & - & - & - & - & - \\
\hline Si IV & 4116.10 & - & - & - & - & 68 & 7.25 & - & - & - & - \\
\hline Si IV & 4212.00 & - & - & - & - & - & - & - & - & - & - \\
\hline
\end{tabular}


I. Hunter et al.: Chemical compositions of B-type stars, Online Material p 56

Table 1. continued.

\begin{tabular}{|c|c|c|c|c|c|c|c|c|c|c|c|}
\hline \multirow[t]{2}{*}{ Species } & \multirow[t]{2}{*}{ Wavelength } & \multicolumn{2}{|c|}{ NGC 2004-087 } & \multicolumn{2}{|c|}{ NGC 2004-088 } & \multicolumn{2}{|c|}{ NGC 2004-090 } & \multicolumn{2}{|c|}{ NGC 2004-091 } & \multicolumn{2}{|c|}{ NGC 2004-094 } \\
\hline & & $E W$ & Abund & $E W$ & Abund & $E W$ & Abund & $E W$ & Abund & $E W$ & Abund \\
\hline C II & 4267.00 & 88 & 7.65 & 166 & 7.65 & 41 & 7.79 & 125 & 8.08 & 68 & 7.04 \\
\hline N II & 3955.85 & - & - & - & - & - & - & - & - & - & - \\
\hline N II & 3995.00 & 32 & 6.88 & $<54$ & $<7.18$ & 40 & 7.72 & 50 & 7.21 & - & - \\
\hline N II & 4447.03 & - & - & - & - & 20 & 7.51 & 33 & 7.39 & - & - \\
\hline N II & 4601.48 & - & - & - & - & - & - & - & - & - & - \\
\hline N II & 4613.86 & - & - & - & - & - & - & 15 & 7.43 & - & - \\
\hline N II & 4630.54 & 24 & 6.98 & - & - & 30 & 7.73 & 34 & 7.20 & $<25$ & $<6.99$ \\
\hline N II & 4643.08 & - & - & - & - & - & - & 16 & 7.22 & - & - \\
\hline O II & 3912.00 & 40 & 8.21 & - & - & 45 & 8.42 & 66 & 8.40 & 38 & 8.51 \\
\hline O II & 3919.30 & - & - & - & - & - & - & - & - & - & - \\
\hline O II & 3945.04 & 23 & 8.16 & - & - & 15 & 8.15 & 42 & 8.41 & - & - \\
\hline O II & 3954.36 & 28 & 8.01 & - & - & 29 & 8.18 & 55 & 8.30 & 39 & 8.56 \\
\hline O II & 3982.71 & 20 & 8.17 & - & - & 22 & 8.38 & 33 & 8.27 & - & - \\
\hline O II & 4069.00 & 43 & 7.61 & - & - & 126 & 8.68 & 121 & 8.31 & - & - \\
\hline O II & 4072.15 & 32 & 7.59 & - & - & 65 & 8.36 & 70 & 8.13 & - & - \\
\hline O II & 4075.86 & 45 & 7.70 & - & - & 66 & 8.19 & 92 & 8.31 & - & - \\
\hline O II & 4078.84 & - & - & - & - & 18 & 8.29 & 37 & 8.43 & - & - \\
\hline O II & 4132.80 & - & - & - & - & 24 & 8.22 & - & - & - & - \\
\hline O II & 4156.53 & - & - & - & - & - & - & - & - & - & - \\
\hline O II & 4185.44 & - & - & - & - & - & - & - & - & - & - \\
\hline O II & 4317.00 & 31 & 7.99 & - & - & 45 & 8.72 & 62 & 8.44 & - & - \\
\hline O II & 4319.63 & 37 & 8.13 & - & - & 40 & 8.63 & 53 & 8.26 & - & - \\
\hline O II & 4325.76 & - & - & - & - & - & - & 22 & 8.44 & - & - \\
\hline O II & 4349.43 & 37 & 7.97 & - & - & - & - & 120 & 9.16 & - & - \\
\hline O II & 4351.00 & - & - & - & - & - & - & 76 & 8.34 & - & - \\
\hline O II & 4353.58 & - & - & - & - & - & - & - & - & - & - \\
\hline O II & 4366.00 & 38 & 8.10 & - & - & 43 & 8.63 & - & - & - & - \\
\hline O II & 4369.27 & - & - & - & - & - & - & - & - & - & - \\
\hline O II & 4395.94 & 24 & 8.36 & - & - & 24 & 8.48 & 35 & 8.45 & - & - \\
\hline O II & 4414.90 & 45 & 7.80 & - & - & 67 & 8.53 & 100 & 8.40 & - & - \\
\hline O II & 4416.98 & 33 & 7.85 & - & - & 51 & 8.57 & 78 & 8.44 & - & - \\
\hline O II & 4443.01 & - & - & - & - & - & - & - & - & - & - \\
\hline O II & 4452.38 & 19 & 8.21 & - & - & - & - & 40 & 8.58 & - & - \\
\hline O II & 4590.97 & 41 & 8.04 & - & - & 51 & 8.36 & 67 & 8.25 & - & - \\
\hline O II & 4596.18 & 49 & 8.27 & - & - & 43 & 8.34 & 62 & 8.23 & 37 & 8.49 \\
\hline O II & 4638.86 & 33 & 7.95 & 47 & 8.56 & 48 & 8.61 & 73 & 8.46 & 40 & 8.52 \\
\hline O II & 4641.81 & 48 & 7.84 & 58 & 8.30 & - & - & 98 & 8.38 & 61 & 8.54 \\
\hline O II & 4650.00 & 97 & 7.89 & 110 & 8.30 & - & - & - & - & 121 & 8.57 \\
\hline O II & 4661.63 & 49 & 8.22 & 58 & 8.66 & 55 & 8.65 & 69 & 8.33 & 39 & 8.44 \\
\hline O II & 4673.73 & - & - & - & - & 15 & 8.75 & 23 & 8.43 & - & - \\
\hline O II & 4676.23 & 28 & 7.89 & - & - & 38 & 8.51 & 60 & 8.31 & - & - \\
\hline O II & 4699.00 & 36 & 7.90 & - & - & 48 & 8.25 & 55 & 8.01 & 35 & 8.35 \\
\hline O II & 4705.35 & 25 & 7.71 & - & - & 47 & 8.29 & 49 & 8.01 & 32 & 8.39 \\
\hline O II & 4710.00 & - & - & - & - & 15 & 8.47 & 36 & 8.81 & - & - \\
\hline Mg II & 4481.00 & 64 & 6.84 & 130 & 6.85 & 44 & 6.97 & 98 & 7.29 & 103 & 6.92 \\
\hline Si II & 4128.05 & - & - & - & - & - & - & - & - & - & - \\
\hline Si II & 4130.89 & - & - & - & - & - & - & - & - & - & - \\
\hline Si III & 4552.62 & 70 & 6.66 & 134 & 7.18 & 68 & 7.19 & 120 & 7.30 & 78 & 7.07 \\
\hline Si III & 4567.84 & 66 & 6.85 & 105 & 7.17 & 51 & 7.15 & 94 & 7.21 & 58 & 6.97 \\
\hline Si III & 4574.75 & 40 & 6.89 & 66 & 7.25 & 23 & 7.12 & 63 & 7.27 & 33 & 6.92 \\
\hline Si IV & 4088.90 & - & - & - & - & - & - & - & - & - & - \\
\hline Si IV & 4116.10 & - & - & - & - & 101 & 7.16 & 44 & 7.26 & - & - \\
\hline Si IV & 4212.00 & - & - & - & - & - & - & - & - & - & - \\
\hline
\end{tabular}


I. Hunter et al.: Chemical compositions of B-type stars, Online Material p 57

Table 1. continued.

\begin{tabular}{|c|c|c|c|c|c|c|c|c|c|c|c|}
\hline \multirow[t]{2}{*}{ Species } & \multirow[t]{2}{*}{ Wavelength } & \multicolumn{2}{|c|}{ NGC 2004-095 } & \multicolumn{2}{|c|}{ NGC 2004-097 } & \multicolumn{2}{|c|}{ NGC 2004-098 } & \multicolumn{2}{|c|}{ NGC 2004-099 } & \multicolumn{2}{|c|}{ NGC 2004-100 } \\
\hline & & $E W$ & Abund & $E W$ & Abund & $E W$ & Abund & $E W$ & Abund & $E W$ & Abund \\
\hline C II & 4267.00 & 90 & 7.71 & 141 & 7.71 & 102 & 7.44 & 125 & 7.61 & 95 & 7.85 \\
\hline N II & 3955.85 & - & - & - & - & - & - & - & - & - & - \\
\hline N II & 3995.00 & - & - & 43 & 6.97 & - & - & 45 & 6.94 & $<60$ & $<7.29$ \\
\hline N II & 4447.03 & - & - & - & - & - & - & - & - & - & - \\
\hline N II & 4601.48 & - & - & - & - & - & - & - & - & - & - \\
\hline N II & 4613.86 & - & - & - & - & - & - & - & - & - & - \\
\hline N II & 4630.54 & 33 & 7.13 & 38 & 7.15 & $<43$ & $<7.34$ & 44 & 7.17 & - & - \\
\hline N II & 4643.08 & - & - & - & - & - & - & - & - & - & - \\
\hline O II & 3912.00 & 52 & 8.28 & 48 & 8.49 & 53 & 8.72 & 65 & 8.63 & - & - \\
\hline O II & 3919.30 & - & - & - & - & - & - & - & - & - & - \\
\hline O II & 3945.04 & - & - & - & - & - & - & - & - & - & - \\
\hline O II & 3954.36 & - & - & - & - & - & - & 52 & 8.44 & - & - \\
\hline O II & 3982.71 & - & - & - & - & - & - & - & - & - & - \\
\hline O II & 4069.00 & 85 & 8.02 & 91 & 8.44 & 82 & 8.48 & - & - & - & - \\
\hline O II & 4072.15 & 64 & 8.11 & 68 & 8.54 & 53 & 8.42 & - & - & - & - \\
\hline O II & 4075.86 & 79 & 8.19 & 81 & 8.57 & 74 & 8.64 & - & - & - & - \\
\hline O II & 4078.84 & - & - & - & - & - & - & - & - & - & - \\
\hline O II & 4132.80 & - & - & - & - & - & - & - & - & - & - \\
\hline O II & 4156.53 & - & - & - & - & - & - & - & - & - & - \\
\hline O II & 4185.44 & - & - & - & - & - & - & - & - & - & - \\
\hline O II & 4317.00 & 60 & 8.47 & 47 & 8.49 & 46 & 8.62 & 50 & 8.40 & 64 & 8.25 \\
\hline O II & 4319.63 & 53 & 8.33 & 48 & 8.51 & 46 & 8.62 & 55 & 8.50 & 62 & 8.22 \\
\hline O II & 4325.76 & - & - & - & - & - & - & - & - & - & - \\
\hline O II & 4349.43 & - & - & - & - & - & - & - & - & - & - \\
\hline O II & 4351.00 & - & - & - & - & - & - & - & - & - & - \\
\hline O II & 4353.58 & - & - & - & - & - & - & - & - & - & - \\
\hline O II & 4366.00 & 61 & 8.43 & 40 & 8.27 & 50 & 8.64 & - & - & - & - \\
\hline O II & 4369.27 & - & - & - & - & - & - & - & - & - & - \\
\hline O II & 4395.94 & - & - & - & - & - & - & - & - & - & - \\
\hline O II & 4414.90 & 94 & 8.41 & 65 & 8.31 & 59 & 8.36 & 63 & 8.14 & - & - \\
\hline O II & 4416.98 & 53 & 8.12 & 67 & 8.65 & 42 & 8.34 & 84 & 8.77 & - & - \\
\hline O II & 4443.01 & - & - & - & - & - & - & - & - & - & - \\
\hline O II & 4452.38 & - & - & 26 & 8.58 & - & - & - & - & - & - \\
\hline O II & 4590.97 & 55 & 8.15 & 42 & 8.29 & 47 & 8.56 & 54 & 8.35 & - & - \\
\hline O II & 4596.18 & 47 & 8.09 & 45 & 8.42 & 49 & 8.64 & 52 & 8.38 & - & - \\
\hline O II & 4638.86 & - & - & 55 & 8.57 & 55 & 8.74 & 71 & 8.68 & - & - \\
\hline O II & 4641.81 & - & - & 72 & 8.41 & 79 & 8.70 & 94 & 8.55 & - & - \\
\hline O II & 4650.00 & 171 & 8.35 & 160 & 8.58 & 162 & 8.78 & 122 & 8.12 & 236 & 8.25 \\
\hline O II & 4661.63 & 49 & 8.07 & 52 & 8.44 & 47 & 8.51 & 73 & 8.63 & 83 & 8.22 \\
\hline O II & 4673.73 & - & - & - & - & - & - & - & - & - & - \\
\hline O II & 4676.23 & 64 & 8.46 & 48 & 8.50 & 36 & 8.40 & 71 & 8.74 & - & - \\
\hline O II & 4699.00 & 57 & 8.09 & 48 & 8.37 & - & - & 46 & 8.20 & - & - \\
\hline O II & 4705.35 & 45 & 8.02 & 40 & 8.32 & 35 & 8.35 & - & - & - & - \\
\hline O II & 4710.00 & - & - & - & - & - & - & - & - & - & - \\
\hline Mg II & 4481.00 & 98 & 7.23 & 118 & 7.06 & 68 & 6.61 & 101 & 6.95 & - & - \\
\hline Si II & 4128.05 & - & - & - & - & - & - & - & - & - & - \\
\hline Si II & 4130.89 & - & - & - & - & - & - & - & - & - & - \\
\hline Si III & 4552.62 & 100 & 7.03 & 124 & 7.33 & 91 & 7.11 & 118 & 7.14 & 170 & 7.13 \\
\hline Si III & 4567.84 & 81 & 7.01 & 82 & 7.05 & 81 & 7.23 & 110 & 7.31 & - & - \\
\hline Si III & 4574.75 & 48 & 6.97 & 61 & 7.26 & 48 & 7.18 & 55 & 7.03 & - & - \\
\hline Si IV & 4088.90 & - & - & - & - & - & - & - & - & - & - \\
\hline Si IV & 4116.10 & - & - & - & - & - & - & - & - & - & - \\
\hline Si IV & 4212.00 & - & - & - & - & - & - & - & - & - & - \\
\hline
\end{tabular}


I. Hunter et al.: Chemical compositions of B-type stars, Online Material $p 58$

Table 1. continued.

\begin{tabular}{|c|c|c|c|c|c|c|c|c|c|c|c|}
\hline \multirow[t]{2}{*}{ Species } & \multirow[t]{2}{*}{ Wavelength } & \multicolumn{2}{|c|}{ NGC 2004-101 } & \multicolumn{2}{|c|}{ NGC 2004-103 } & \multicolumn{2}{|c|}{ NGC 2004-104 } & \multicolumn{2}{|c|}{ NGC 2004-105 } & \multicolumn{2}{|c|}{ NGC 2004-106 } \\
\hline & & $E W$ & Abund & $E W$ & Abund & $E W$ & Abund & $E W$ & Abund & $E W$ & Abund \\
\hline C II & 4267.00 & 133 & 7.65 & 143 & 7.71 & 119 & 7.97 & 125 & 8.00 & 153 & 7.79 \\
\hline N II & 3955.85 & - & - & - & - & - & - & - & - & - & - \\
\hline N II & 3995.00 & 85 & 7.47 & 24 & 6.73 & $<60$ & $<7.28$ & $<50$ & $<7.11$ & 48 & 7.01 \\
\hline N II & 4447.03 & - & - & - & - & - & - & - & - & - & - \\
\hline N II & 4601.48 & - & - & - & - & - & - & - & - & - & - \\
\hline $\mathrm{N}$ II & 4613.86 & - & - & - & - & - & - & - & - & - & - \\
\hline N II & 4630.54 & 84 & 7.72 & 31 & 7.18 & - & - & - & - & 39 & 7.13 \\
\hline N II & 4643.08 & - & - & - & - & - & - & - & - & - & - \\
\hline O II & 3912.00 & - & - & - & - & - & - & - & - & 48 & 8.43 \\
\hline O II & 3919.30 & - & - & - & - & - & - & - & - & - & - \\
\hline O II & 3945.04 & - & - & - & - & - & - & - & - & - & - \\
\hline O II & 3954.36 & 57 & 8.52 & 21 & 8.12 & - & - & 43 & 8.04 & 36 & 8.19 \\
\hline O II & 3982.71 & - & - & - & - & - & - & - & - & - & - \\
\hline O II & 4069.00 & - & - & 74 & 8.50 & - & - & - & - & 86 & 8.30 \\
\hline O II & 4072.15 & - & - & 37 & 8.17 & - & - & - & - & 53 & 8.17 \\
\hline O II & 4075.86 & - & - & 38 & 8.02 & - & - & - & - & 65 & 8.22 \\
\hline O II & 4078.84 & - & - & 23 & 8.64 & - & - & - & - & 34 & 8.66 \\
\hline O II & 4132.80 & - & - & - & - & - & - & - & - & - & - \\
\hline O II & 4156.53 & - & - & - & - & - & - & - & - & - & - \\
\hline O II & 4185.44 & - & - & - & - & - & - & - & - & - & - \\
\hline O II & 4317.00 & 37 & 8.13 & 21 & 8.06 & - & - & - & - & 57 & 8.61 \\
\hline O II & 4319.63 & 62 & 8.62 & 20 & 8.03 & - & - & - & - & 50 & 8.47 \\
\hline O II & 4325.76 & - & - & - & - & - & - & - & - & - & - \\
\hline O II & 4349.43 & - & - & - & - & - & - & - & - & - & - \\
\hline O II & 4351.00 & - & - & - & - & - & - & - & - & - & - \\
\hline O II & 4353.58 & - & - & - & - & - & - & - & - & - & - \\
\hline O II & 4366.00 & 57 & 8.46 & - & - & - & - & - & - & 62 & 8.64 \\
\hline O II & 4369.27 & - & - & - & - & - & - & - & - & - & - \\
\hline O II & 4395.94 & - & - & 29 & 8.97 & - & - & - & - & 43 & 8.97 \\
\hline O II & 4414.90 & 54 & 8.00 & 59 & 8.48 & 59 & 7.83 & - & - & 77 & 8.42 \\
\hline O II & 4416.98 & 66 & 8.48 & 32 & 8.21 & 58 & 8.10 & - & - & 76 & 8.73 \\
\hline O II & 4443.01 & - & - & - & - & - & - & - & - & - & - \\
\hline O II & 4452.38 & - & - & - & - & - & - & - & - & - & - \\
\hline O II & 4590.97 & 82 & 8.80 & - & - & - & - & - & - & 55 & 8.46 \\
\hline O II & 4596.18 & - & - & - & - & - & - & - & - & 52 & 8.46 \\
\hline O II & 4638.86 & 44 & 8.21 & - & - & - & - & - & - & 52 & 8.44 \\
\hline O II & 4641.81 & 96 & 8.55 & 53 & 8.40 & - & - & - & - & 73 & 8.34 \\
\hline O II & 4650.00 & 204 & 8.69 & 102 & 8.40 & 190 & 8.32 & 210 & 8.38 & 149 & 8.42 \\
\hline O II & 4661.63 & 58 & 8.39 & 15 & 7.75 & - & - & - & - & - & - \\
\hline O II & 4673.73 & - & - & - & - & - & - & - & - & 30 & 8.89 \\
\hline O II & 4676.23 & 61 & 8.57 & - & - & - & - & - & - & 58 & 8.60 \\
\hline O II & 4699.00 & 54 & 8.34 & - & - & - & - & - & - & 51 & 8.35 \\
\hline O II & 4705.35 & - & - & - & - & - & - & - & - & 35 & 8.15 \\
\hline O II & 4710.00 & - & - & - & - & - & - & - & - & - & - \\
\hline Mg II & 4481.00 & 107 & 6.96 & 110 & 6.98 & 114 & 7.36 & 84 & 7.06 & 121 & 7.10 \\
\hline $\mathrm{Si}$ II & 4128.05 & - & - & - & - & - & - & - & - & - & - \\
\hline Si II & 4130.89 & - & - & - & - & - & - & - & - & - & - \\
\hline Si III & 4552.62 & 111 & 6.98 & 90 & 7.20 & 128 & 7.23 & 138 & 7.23 & 105 & 7.04 \\
\hline Si III & 4567.84 & 105 & 7.17 & 68 & 7.11 & - & - & - & - & 103 & 7.28 \\
\hline Si III & 4574.75 & 76 & 7.33 & 47 & 7.25 & - & - & - & - & 61 & 7.20 \\
\hline Si IV & 4088.90 & - & - & - & - & - & - & - & - & - & - \\
\hline Si IV & 4116.10 & - & - & - & - & - & - & - & - & - & - \\
\hline Si IV & 4212.00 & - & - & - & - & - & - & - & - & - & - \\
\hline
\end{tabular}


I. Hunter et al.: Chemical compositions of B-type stars, Online Material p 59

Table 1. continued.

\begin{tabular}{|c|c|c|c|c|c|c|c|c|c|c|c|}
\hline \multirow[t]{2}{*}{ Species } & \multirow[t]{2}{*}{ Wavelength } & \multicolumn{2}{|c|}{ NGC 2004-107 } & \multicolumn{2}{|c|}{ NGC 2004-108 } & \multicolumn{2}{|c|}{ NGC 2004-109 } & \multicolumn{2}{|c|}{ NGC 2004-110 } & \multicolumn{2}{|c|}{ NGC 2004-111 } \\
\hline & & $E W$ & Abund & $E W$ & Abund & $E W$ & Abund & $E W$ & Abund & $E W$ & Abund \\
\hline C II & 4267.00 & 87 & 7.94 & 103 & 7.56 & 163 & 7.76 & 128 & 7.62 & 140 & 7.56 \\
\hline N II & 3955.85 & - & - & - & - & - & - & - & - & - & - \\
\hline N II & 3995.00 & $<34$ & $<7.12$ & 36 & 6.94 & 29 & 6.82 & 42 & 6.88 & - & - \\
\hline N II & 4447.03 & - & - & 13 & 6.91 & - & - & - & - & - & - \\
\hline N II & 4601.48 & - & - & - & - & - & - & - & - & - & - \\
\hline N II & 4613.86 & - & - & - & - & - & - & - & - & - & - \\
\hline N II & 4630.54 & - & - & 18 & 6.83 & 19 & 6.88 & 36 & 7.03 & 34 & 7.10 \\
\hline N II & 4643.08 & - & - & - & - & - & - & - & - & - & - \\
\hline O II & 3912.00 & 64 & 8.36 & 32 & 8.30 & - & - & - & - & - & - \\
\hline O II & 3919.30 & - & - & - & - & - & - & - & - & - & - \\
\hline O II & 3945.04 & - & - & 20 & 8.27 & - & - & - & - & - & - \\
\hline O II & 3954.36 & 69 & 8.50 & 35 & 8.41 & 33 & 8.45 & 56 & 8.47 & 39 & 8.40 \\
\hline O II & 3982.71 & - & - & 15 & 8.19 & - & - & - & - & - & - \\
\hline O II & 4069.00 & - & - & 63 & 8.20 & 51 & 8.16 & - & - & 84 & 8.46 \\
\hline O II & 4072.15 & - & - & 36 & 8.02 & 33 & 8.08 & - & - & 64 & 8.58 \\
\hline O II & 4075.86 & - & - & 47 & 8.10 & 54 & 8.42 & - & - & - & - \\
\hline O II & 4078.84 & - & - & 12 & 8.05 & - & - & - & - & - & - \\
\hline O II & 4132.80 & - & - & 16 & 8.05 & - & - & - & - & - & - \\
\hline O II & 4156.53 & - & - & - & - & - & - & - & - & - & - \\
\hline O II & 4185.44 & - & - & - & - & - & - & - & - & - & - \\
\hline O II & 4317.00 & 45 & 8.21 & 32 & 8.27 & 35 & 8.47 & 48 & 8.33 & 36 & 8.31 \\
\hline O II & 4319.63 & 58 & 8.44 & 35 & 8.36 & 38 & 8.55 & 52 & 8.40 & 45 & 8.52 \\
\hline O II & 4325.76 & - & - & - & - & - & - & - & - & - & - \\
\hline O II & 4349.43 & - & - & 35 & 8.21 & - & - & - & - & - & - \\
\hline O II & 4351.00 & - & - & 24 & 8.13 & - & - & - & - & - & - \\
\hline O II & 4353.58 & - & - & - & - & - & - & - & - & - & - \\
\hline O II & 4366.00 & 68 & 8.56 & 27 & 8.09 & - & - & 65 & 8.56 & - & - \\
\hline O II & 4369.27 & - & - & - & - & - & - & - & - & - & - \\
\hline O II & 4395.94 & - & - & 21 & 8.57 & - & - & - & - & - & - \\
\hline O II & 4414.90 & 77 & 8.15 & 52 & 8.22 & 44 & 8.21 & - & - & 59 & 8.31 \\
\hline O II & 4416.98 & 95 & 8.67 & 46 & 8.42 & 39 & 8.41 & - & - & 39 & 8.23 \\
\hline O II & 4443.01 & - & - & - & - & - & - & - & - & - & - \\
\hline O II & 4452.38 & - & - & 15 & 8.30 & - & - & - & - & - & - \\
\hline O II & 4590.97 & 86 & 8.49 & 29 & 8.11 & 31 & 8.34 & 71 & 8.59 & - & - \\
\hline O II & 4596.18 & 81 & 8.45 & 32 & 8.27 & 34 & 8.48 & 61 & 8.49 & - & - \\
\hline O II & 4638.86 & 73 & 8.48 & 35 & 8.30 & 33 & 8.40 & 84 & 8.82 & 36 & 8.27 \\
\hline O II & 4641.81 & 109 & 8.53 & 47 & 8.15 & 47 & 8.29 & 114 & 8.73 & 54 & 8.20 \\
\hline O II & 4650.00 & - & - & - & - & 101 & 8.40 & 270 & 9.04 & 122 & 8.38 \\
\hline O II & 4661.63 & - & - & 35 & 8.24 & 30 & 8.25 & 84 & 8.74 & 52 & 8.54 \\
\hline O II & 4673.73 & - & - & 11 & 8.38 & - & - & - & - & - & - \\
\hline O II & 4676.23 & 72 & 8.52 & 26 & 8.12 & - & - & - & - & 40 & 8.42 \\
\hline O II & 4699.00 & 93 & 8.36 & 24 & 7.97 & - & - & - & - & 32 & 8.18 \\
\hline O II & 4705.35 & 55 & 8.08 & 30 & 8.18 & 32 & 8.45 & 67 & 8.61 & - & - \\
\hline O II & 4710.00 & - & - & 13 & 8.63 & - & - & - & - & - & - \\
\hline Mg II & 4481.00 & 70 & 7.12 & 98 & 6.99 & 138 & 7.15 & 115 & 7.03 & 106 & 6.88 \\
\hline Si II & 4128.05 & - & - & 20 & 7.21 & - & - & - & - & - & - \\
\hline Si II & 4130.89 & - & - & 18 & 6.87 & - & - & - & - & - & - \\
\hline Si III & 4552.62 & 93 & 7.04 & 76 & 6.95 & 88 & 7.19 & 116 & 7.00 & 95 & 7.04 \\
\hline Si III & 4567.84 & - & - & 62 & 6.97 & 70 & 7.16 & 106 & 7.15 & 88 & 7.22 \\
\hline Si III & 4574.75 & - & - & 39 & 7.02 & 46 & 7.24 & 86 & 7.45 & 56 & 7.24 \\
\hline Si IV & 4088.90 & - & - & - & - & - & - & - & - & - & - \\
\hline Si IV & 4116.10 & - & - & - & - & - & - & - & - & - & - \\
\hline Si IV & 4212.00 & - & - & - & - & - & - & - & - & - & - \\
\hline
\end{tabular}


I. Hunter et al.: Chemical compositions of B-type stars, Online Material p 60

Table 1. continued.

\begin{tabular}{|c|c|c|c|c|c|c|c|c|c|c|c|}
\hline \multirow[t]{2}{*}{ Species } & \multirow[t]{2}{*}{ Wavelength } & \multicolumn{2}{|c|}{ NGC 2004-112 } & \multicolumn{2}{|c|}{ NGC 2004-113 } & \multicolumn{2}{|c|}{ NGC 2004-114 } & \multicolumn{2}{|c|}{ NGC 2004-116 } & \multicolumn{2}{|c|}{ NGC 2004-117 } \\
\hline & & $E W$ & Abund & $E W$ & Abund & $E W$ & Abund & $E W$ & Abund & $E W$ & Abund \\
\hline C II & 4267.00 & 120 & 7.57 & 148 & 7.56 & 127 & 7.61 & 128 & 7.61 & 126 & 7.58 \\
\hline N II & 3955.85 & - & - & - & - & - & - & - & - & - & - \\
\hline N II & 3995.00 & - & - & $<70$ & $<7.29$ & 61 & 7.25 & 38 & 6.86 & 38 & 6.86 \\
\hline N II & 4447.03 & - & - & - & - & - & - & - & - & - & - \\
\hline N II & 4601.48 & - & - & - & - & - & - & - & - & - & - \\
\hline $\mathrm{N}$ II & 4613.86 & - & - & - & - & - & - & - & - & - & - \\
\hline N II & 4630.54 & $<21$ & $<6.85$ & - & - & 25 & 6.91 & 31 & 7.00 & 54 & 7.36 \\
\hline N II & 4643.08 & - & - & - & - & - & - & - & - & - & - \\
\hline O II & 3912.00 & - & - & - & - & 34 & 8.23 & 70 & 8.77 & 58 & 8.60 \\
\hline O II & 3919.30 & - & - & - & - & - & - & - & - & - & - \\
\hline O II & 3945.04 & - & - & - & - & - & - & 39 & 8.59 & - & - \\
\hline O II & 3954.36 & - & - & - & - & 50 & 8.55 & 47 & 8.42 & 50 & 8.48 \\
\hline O II & 3982.71 & - & - & - & - & - & - & 47 & 8.81 & - & - \\
\hline O II & 4069.00 & - & - & - & - & 77 & 8.26 & 119 & 8.66 & 125 & 8.71 \\
\hline O II & 4072.15 & - & - & - & - & 47 & 8.12 & 77 & 8.59 & 81 & 8.63 \\
\hline O II & 4075.86 & - & - & - & - & 55 & 8.11 & 93 & 8.65 & 94 & 8.63 \\
\hline O II & 4078.84 & - & - & - & - & 21 & 8.33 & 40 & 8.81 & - & - \\
\hline O II & 4132.80 & - & - & - & - & - & - & - & - & - & - \\
\hline O II & 4156.53 & - & - & - & - & - & - & - & - & - & - \\
\hline O II & 4185.44 & - & - & - & - & - & - & - & - & - & - \\
\hline O II & 4317.00 & 43 & 8.47 & - & - & 44 & 8.42 & 56 & 8.59 & 71 & 8.83 \\
\hline O II & 4319.63 & 37 & 8.33 & - & - & 49 & 8.53 & 54 & 8.55 & 59 & 8.63 \\
\hline O II & 4325.76 & - & - & - & - & - & - & - & - & - & - \\
\hline O II & 4349.43 & - & - & - & - & - & - & 63 & 8.42 & - & - \\
\hline O II & 4351.00 & - & - & - & - & - & - & 47 & 8.38 & - & - \\
\hline O II & 4353.58 & - & - & - & - & - & - & - & - & - & - \\
\hline O II & 4366.00 & - & - & - & - & 38 & 8.22 & 61 & 8.60 & 68 & 8.70 \\
\hline O II & 4369.27 & - & - & - & - & - & - & - & - & - & - \\
\hline O II & 4395.94 & - & - & - & - & - & - & - & - & - & - \\
\hline O II & 4414.90 & - & - & - & - & 62 & 8.26 & 80 & 8.45 & 99 & 8.68 \\
\hline O II & 4416.98 & - & - & - & - & 47 & 8.30 & 71 & 8.63 & 83 & 8.78 \\
\hline O II & 4443.01 & - & - & - & - & - & - & - & - & - & - \\
\hline O II & 4452.38 & - & - & - & - & - & - & 29 & 8.61 & 40 & 8.87 \\
\hline O II & 4590.97 & 36 & 8.23 & - & - & 38 & 8.20 & 61 & 8.56 & 51 & 8.39 \\
\hline O II & 4596.18 & 45 & 8.49 & - & - & 41 & 8.34 & 88 & 8.98 & 55 & 8.53 \\
\hline O II & 4638.86 & 51 & 8.58 & - & - & 52 & 8.51 & 55 & 8.49 & 54 & 8.47 \\
\hline O II & 4641.81 & 66 & 8.41 & - & - & 40 & 7.85 & 81 & 8.44 & 96 & 8.61 \\
\hline O II & 4650.00 & 119 & 8.34 & 134 & 8.30 & 132 & 8.36 & 211 & 8.82 & 189 & 8.66 \\
\hline O II & 4661.63 & 50 & 8.49 & - & - & 45 & 8.31 & 76 & 8.75 & - & - \\
\hline O II & 4673.73 & - & - & - & - & - & - & - & - & - & - \\
\hline O II & 4676.23 & - & - & - & - & - & - & - & - & - & - \\
\hline O II & 4699.00 & - & - & - & - & - & - & - & - & - & - \\
\hline O II & 4705.35 & - & - & - & - & 35 & 8.21 & 43 & 8.32 & - & - \\
\hline O II & 4710.00 & - & - & - & - & - & - & - & - & - & - \\
\hline Mg II & 4481.00 & 92 & 6.86 & 140 & 7.03 & 110 & 6.99 & 96 & 6.85 & 123 & 7.03 \\
\hline Si II & 4128.05 & - & - & - & - & - & - & - & - & - & - \\
\hline Si II & 4130.89 & - & - & - & - & - & - & - & - & - & - \\
\hline Si III & 4552.62 & 93 & 7.08 & 135 & 7.20 & 107 & 7.13 & 130 & 7.26 & 139 & 7.30 \\
\hline Si III & 4567.84 & 83 & 7.19 & - & - & 91 & 7.18 & 97 & 7.14 & 102 & 7.15 \\
\hline Si III & 4574.75 & 55 & 7.26 & - & - & 60 & 7.24 & 70 & 7.30 & 66 & 7.20 \\
\hline Si IV & 4088.90 & - & - & - & - & - & - & - & - & - & - \\
\hline Si IV & 4116.10 & - & - & - & - & - & - & - & - & - & - \\
\hline Si IV & 4212.00 & - & - & - & - & - & - & - & - & - & - \\
\hline
\end{tabular}


I. Hunter et al.: Chemical compositions of B-type stars, Online Material p 61

Table 1. continued.

\begin{tabular}{|c|c|c|c|}
\hline \multirow[t]{2}{*}{$\overline{\text { Species }}$} & \multirow[t]{2}{*}{ Wavelength } & \multicolumn{2}{|c|}{$\overline{\text { NGC 2004-119 }}$} \\
\hline & & $E W$ & Abund \\
\hline C II & 4267.00 & 123 & 7.78 \\
\hline N II & 3955.85 & 7 & 6.97 \\
\hline N II & 3995.00 & 40 & 6.91 \\
\hline N II & 4447.03 & - & - \\
\hline N II & 4601.48 & - & - \\
\hline N II & 4613.86 & - & - \\
\hline N II & 4630.54 & - & - \\
\hline N II & 4643.08 & 13 & 6.96 \\
\hline O II & 3912.00 & 50 & 8.39 \\
\hline O II & 3919.30 & - & - \\
\hline O II & 3945.04 & 36 & 8.46 \\
\hline O II & 3954.36 & 46 & 8.35 \\
\hline O II & 3982.71 & 25 & 8.23 \\
\hline O II & 4069.00 & 88 & 8.24 \\
\hline O II & 4072.15 & 53 & 8.10 \\
\hline O II & 4075.86 & 67 & 8.20 \\
\hline O II & 4078.84 & 21 & 8.18 \\
\hline O II & 4132.80 & 37 & 8.43 \\
\hline O II & 4156.53 & - & - \\
\hline O II & 4185.44 & - & - \\
\hline O II & 4317.00 & 63 & 8.69 \\
\hline O II & 4319.63 & 60 & 8.62 \\
\hline O II & 4325.76 & 21 & 8.54 \\
\hline O II & 4349.43 & 63 & 8.40 \\
\hline O II & 4351.00 & 38 & 8.08 \\
\hline O II & 4353.58 & - & - \\
\hline $\mathrm{O}$ II & 4366.00 & 49 & 8.34 \\
\hline O II & 4369.27 & 15 & 8.27 \\
\hline O II & 4395.94 & 43 & 8.89 \\
\hline O II & 4414.90 & 65 & 8.17 \\
\hline O II & 4416.98 & - & - \\
\hline O II & 4443.01 & - & - \\
\hline O II & 4452.38 & 33 & 8.62 \\
\hline O II & 4590.97 & 46 & 8.18 \\
\hline O II & 4596.18 & 59 & 8.47 \\
\hline O II & 4638.86 & 52 & 8.36 \\
\hline O II & 4641.81 & 66 & 8.17 \\
\hline O II & 4650.00 & - & - \\
\hline O II & 4661.63 & 53 & 8.31 \\
\hline O II & 4673.73 & 18 & 8.43 \\
\hline O II & 4676.23 & 46 & 8.29 \\
\hline O II & 4699.00 & 40 & 8.03 \\
\hline O II & 4705.35 & 38 & 8.08 \\
\hline O II & 4710.00 & 21 & 8.68 \\
\hline Mg II & 4481.00 & 108 & 7.17 \\
\hline Si II & 4128.05 & 19 & 7.27 \\
\hline Si II & 4130.89 & 22 & 7.13 \\
\hline Si III & 4552.62 & 100 & 7.09 \\
\hline Si III & 4567.84 & 85 & 7.13 \\
\hline Si III & 4574.75 & 58 & 7.21 \\
\hline Si IV & 4088.90 & - & - \\
\hline Si IV & 4116.10 & - & - \\
\hline Si IV & 4212.00 & - & - \\
\hline
\end{tabular}


I. Hunter et al.: Chemical compositions of B-type stars, Online Material p 62

Table 2. Atmospheric parameters, projected rotational velocities and chemical compositions of the sample.

\begin{tabular}{|c|c|c|c|c|c|c|c|c|c|}
\hline Star & $\begin{array}{l}T_{\text {eff }} \\
(\mathrm{K})\end{array}$ & $\begin{array}{l}\log g \\
(\operatorname{dex})\end{array}$ & $\begin{array}{c}\xi_{\mathrm{vt}} \\
\left(\mathrm{km} \mathrm{s}^{-1}\right)\end{array}$ & $\begin{array}{c}v \sin i \\
\left(\mathrm{~km} \mathrm{~s}^{-1}\right)\end{array}$ & $\begin{array}{c}\text { Carbon } \\
\text { (dex) }\end{array}$ & $\begin{array}{l}\text { Nitrogen } \\
\text { (dex) }\end{array}$ & $\begin{array}{l}\text { Oxygen } \\
\text { (dex) }\end{array}$ & $\begin{array}{l}\text { Magnesium } \\
\text { (dex) }\end{array}$ & $\begin{array}{c}\text { Silicon } \\
\text { (dex) }\end{array}$ \\
\hline NGC 6611-001 & 30300 & 3.70 & 10 & 142 & $8.06 \pm 0.29(1)$ & $7.87 \pm 0.20(4)$ & $8.36 \pm 0.20(3)$ & $7.36 \pm 0.25(1)$ & $7.47 \pm 0.24(3)$ \\
\hline NGC 6611-006 & 31250 & 4.00 & 8 & 20 & $8.14 \pm 0.26(1)$ & $7.58 \pm 0.10(3)$ & $8.50 \pm 0.12(28)$ & $7.36 \pm 0.21$ & $7.41 \pm 0.20(3)$ \\
\hline NGC 6611-012 & 27200 & 3.90 & 5 & 95 & $8.19 \pm 0.24$ & $7.50 \pm 0.23$ & $8.55 \pm 0.14(23)$ & $7.26 \pm 0.22(1)$ & $7.45 \pm 0.30$ \\
\hline NGC 6611-020 & 27000 & 4.10 & 2 & 220 & $7.98 \pm 0.28$ & $7.88 \pm 0.22(2)$ & $8.41 \pm 0.15(6)$ & $7.60 \pm 0.27$ & $7.47 \pm 0.28$ \\
\hline NGC 6611-021 & 26250 & 4.25 & 0 & 30 & $7.92 \pm 0.22(1)$ & $7.51 \pm 0.12(3)$ & $8.60 \pm 0.16(24)$ & & $31(3)$ \\
\hline & 25000 & 4.10 & 0 & 95 & $8.19 \pm 0.33(1)$ & $7.84 \pm 0.16$ & & & (3) \\
\hline NGC & 22500 & 4.15 & 1 & 10 & $8.35 \pm 0.21(1)$ & $7.69 \pm 0.16(5)$ & $8.57 \pm 0.2$ & $7.32 \pm 0.24(1)$ & $7.46 \pm 0.28$ \\
\hline NGC 6611 & 22500 & 4.15 & - & 70 & $8.32 \pm 0.24(1)$ & $7.73 \pm 0.22(5)$ & $8.46 \pm 0.32$ & - & $7.28 \pm 0.33(3)$ \\
\hline NGC & 25600 & 4.00 & 4 & 30 & $8.19 \pm 0.18$ & $7.70 \pm 0.13$ & $8.54 \pm 0.14$ & $7.38 \pm 0.18$ & $7.43 \pm 0.30(3)$ \\
\hline NGC 6611 & 27000 & 4.10 & 2 & 120 & $8.00 \pm 0.22(1)$ & $7.75 \pm 0.16$ & $8.63 \pm 0.15(9)$ & - & $7.47 \pm 0.29$ \\
\hline NGC 6611-04 & 22500 & 3.90 & 4 & 155 & $8.03 \pm 0.26(1)$ & $7.67 \pm 0.21$ & $8.61 \pm 0.33(14)$ & $7.57 \pm 0.32(1)$ & $7.48 \pm 0.36(3)$ \\
\hline & 19200 & 3.85 & 8 & 120 & $7.74 \pm 0.22(1)$ & $7.72 \pm 0.30(2)$ & & $6.86 \pm 0.20$ & $7.42 \pm 0.37(3)$ \\
\hline & 18400 & 4.05 & 7 & 165 & $7.91 \pm 0.31$ & $7.48 \pm 0.34$ & $8.73 \pm 0.45$ & $7.04 \pm 0.27$ & $7.42 \pm 0.42(1)$ \\
\hline & 22500 & 4.00 & 2 & 95 & $8.19 \pm 0.22(1)$ & $7.57 \pm 0.24(2)$ & $8.82 \pm 0.40(1)$ & $7.34 \pm 0.27(1)$ & $7.49 \pm 0.34$ \\
\hline & 20800 & 4.20 & - & 80 & $8.18 \pm 0.26(1)$ & $7.60 \pm 0.29(2)$ & $8.78 \pm 0.37$ (2) & $7.43 \pm 0.28$ & $7.34 \pm 0.38$ \\
\hline NGC 3 & 26600 & 3.05 & 23 & 80 & $8.01 \pm 0.34(1)$ & $7.85 \pm 0.16(5)$ & $8.44 \pm 0.19(15)$ & $7.33 \pm 0.29$ & $7.47 \pm$ \\
\hline & 23200 & 2.80 & 15 & 100 & $8.10 \pm 0.22(1)$ & $8.08 \pm 0.12(5)$ & $8.67 \pm 0.15(16)$ & $7.56 \pm 0.21(1)$ & $7.44 \pm 0.27$ \\
\hline & 20500 & 2.75 & 13 & 80 & $7.95 \pm 0.22(1)$ & $7.52 \pm 0.06$ & $8.75 \pm 0.20$ & & \\
\hline & 22700 & 3.13 & 13 & 105 & $8.17 \pm 0$ & $7.55 \pm 0.09$ & & & \\
\hline & 21500 & 3.05 & 14 & 195 & $7.89 \pm$ & $7.56 \pm$ & & & \\
\hline & 21500 & 3.15 & 15 & 200 & $7.81 \pm$ & $7.37 \pm$ & $8.84 \pm$ & 7.19 & $7.46 \pm$ \\
\hline NGC & 22600 & 3.10 & 11 & 65 & $8.13 \pm$ & $7.50 \pm$ & $8.71 \pm 0$ & $7.26 \pm$ & $.44 \pm$ \\
\hline NGC & 21500 & 3.25 & 12 & 140 & $7.83 \pm 0.2$ & $7.59 \pm 0.1$ & $8.77 \pm$ & $7.20 \pm$ & $1.40 \pm$ \\
\hline NGC & 21450 & 3.20 & 11 & 70 & $7.85 \pm 0.2$ & $7.45 \pm 0.08(6)$ & $8.82 \pm$ & $7.17 \pm 0.22(1)$ & $7.49 \pm 0.34(3)$ \\
\hline NG & 21500 & 3.30 & 11 & 100 & $7.86 \pm 0.17(1)$ & $7.45 \pm 0.13$ & $8.83 \pm 0.2$ & $7.29 \pm 0.2$ & $7.50 \pm 0.34$ \\
\hline & 19200 & 4.00 & 1 & 110 & $8.13 \pm 0.29(1)$ & $7.66 \pm 0.33$ & $8.58 \pm 0.45$ & $7.35 \pm 0.31$ & $7.44 \pm 0.38$ \\
\hline & 23450 & 3.75 & 5 & 26 & $7.96 \pm 0.16(1)$ & $7.56 \pm 0.08$ & $8.71 \pm 0$ & $7.15 \pm 0.1$ & $7.49 \pm 0.3$ \\
\hline & 25000 & 3.85 & 6 & 120 & $8.26 \pm 0$ & $7.59 \pm 0.25$ & $8.58 \pm 0$ & $7.51 \pm 0$ & $7.41 \pm 0$ \\
\hline & 20500 & 3.40 & 6 & 50 & $7.72 \pm$ & $7.57 \pm 0$ & $8.69 \pm$ & $7.04 \pm 0$ & $7.43 \pm$ \\
\hline & 20500 & 3.50 & 6 & & $7.75 \pm$ & $7.62 \pm$ & $8.78 \pm$ & $7.06 \pm$ & (3) \\
\hline & 21100 & 3.70 & 5 & 215 & 7.8 & $7.41 \pm($ & $8.70=$ & 6.99 & 3) \\
\hline & 22100 & 3.65 & 2 & 30 & $8.03 \pm$ & $7.66 \pm$ & $8.75 \pm$ & 7.22 & (3) \\
\hline & 19400 & 3.65 & 7 & 215 & $7.69 \pm$ & $7.52 \pm$ & $8.69 \pm$ & $6.96=$ & (3) \\
\hline & 19800 & 3.70 & 4 & 05 & $7.86 \pm$ & $7.57 \pm($ & $8.65 \pm 0.37$ (4) & $6.99 \pm 0.27(1)$ & 3) \\
\hline & 17400 & 3.45 & 12 & 30 & 7.6 & $7.70 \pm$ & - & - & (3) \\
\hline & 26100 & 4.25 & - & 35 & $8.28 \pm$ & $7.60 \pm 0.11(7)$ & 8.13 & $7.10 \pm 0$ & $7.10 \pm$ \\
\hline & 20600 & 3.80 & 5 & 250 & $7.92 \pm$ & $7.64 \pm$ & $8.54 \pm$ & $7.24 \pm$ & $7.43 \pm$ \\
\hline NGC & 20800 & 3.95 & 3 & 70 & $7.99 \pm($ & $7.77 \pm 0.26$ & $8.73 \pm 0.34(18)$ & $7.14 \pm 0.21$ & $7.42 \pm 0.35$ \\
\hline NGC & 20600 & 3.95 & 1 & 235 & $8.16 \pm 0.26(1)$ & $7.57 \pm 0.29(2)$ & $8.61 \pm 0.41$ & $7.24 \pm 0.29$ & $7.47 \pm 0.36$ \\
\hline NGC 3293-041 & 20600 & 4.00 & 0 & 205 & $8.34 \pm 0.27$ (1) & $7.53 \pm 0.29(2)$ & $8.62 \pm 0.38(2)$ & $7.33 \pm 0.29$ & $7.18 \pm 0.40(1)$ \\
\hline NGC & 19500 & 4.05 & - & 14 & $7.94 \pm 0.24$ & $7.55 \pm 0.19(7)$ & $8.50 \pm 0.25(19)$ & $7.14 \pm 0.26$ & $7.31 \pm 0.28$ \\
\hline NGC & 17900 & 4.20 & 1 & 24 & $8.07 \pm 0.27(1)$ & $7.78 \pm 0.25(2)$ & $8.31 \pm 0.31(1)$ & $7.11 \pm 0.28$ & $7.44 \pm 0.30(2)$ \\
\hline-002 & 15950 & 2.20 & 18 & 70 & $7.72 \pm 0.28$ & $8.18 \pm 0.24(7)$ & $8.59 \pm 0 . ?$ & $7.30 \pm 0.28$ & $7.40 \pm 0.35$ \\
\hline NGC & 17700 & 2.50 & 15 & 38 & $7.70 \pm 0.20$ & $8.12 \pm 0.21(7)$ & $8.52 \pm 0.2$ & $7.33 \pm 0.23$ & $7.40 \pm 0.32(3)$ \\
\hline-004 & 19550 & 2.60 & 17 & 75 & $7.79 \pm 0.2$ & $8.07 \pm 0.09(7)$ & $8.64 \pm 0$ & $7.29 \pm 0.22(1)$ & $7.38 \pm 0.24$ \\
\hline & 19900 & 2.95 & 17 & 100 & (1) & 7.6 & & $7.21=$ & $.27(3)$ \\
\hline & 22400 & 3.70 & 5 & 48 & $2(1)$ & $7.81 \pm 0.17(5)$ & $8.80 \pm 0$ & $7.09 \pm 0.23$ & $7.47 \pm 0.33(3)$ \\
\hline & 22500 & 3.80 & 5 & 195 & $5(1)$ & $7.64 \pm 0.39$ & $8.63 \pm 0$ & - & $7.46 \pm 0.37(3)$ \\
\hline & 20400 & 3.90 & 3 & 5 & (1) & $7.74 \pm$ & $8.71 \pm($ & $7.32 \pm 0$ & $8(3)$ \\
\hline & 21700 & 3.95 & 1 & 20 & $5(1)$ & $7.68 \pm$ & $8.51 \pm 0$ & 7.44 & $7.39 \pm$ \\
\hline & 19800 & 3.95 & 1 & 120 & 2(1) & $7.77 \pm 0$ & $8.82 \pm 0.39$ & $7.41 \pm 0.2$ & $7.44 \pm$ \\
\hline & 18600 & 3.95 & 6 & 205 & $.32(1)$ & $7.47 \pm 0.34(1)$ & - & $6.93 \pm 0.26(1)$ & $7.46 \pm 0.43(1)$ \\
\hline 5-029 & 20200 & 4.00 & 0 & 120 & $8.21 \pm 0.34(1)$ & $7.67 \pm 0.28$ & $8.48 \pm 0.34(8)$ & $7.45 \pm 0.35$ & $7.41 \pm 0.36(3)$ \\
\hline & 17300 & 3.85 & 6 & 75 & $8.07 \pm 0.32(1)$ & $7.43 \pm 0.28(2)$ & $8.68 \pm 0.33(3)$ & $7.09 \pm 0.28$ & $7.34 \pm 0.28$ \\
\hline & 18900 & 4.10 & 2 & 65 & $8.29 \pm 0.39(1)$ & $7.69 \pm 0.34(2)$ & $8.98 \pm 0.34$ (3) & $7.19 \pm 0.40$ & $7.44 \pm 0.28$ \\
\hline $4755-048$ & 17800 & 3.95 & 4 & 55 & $8.22 \pm 0.32(1)$ & $7.45 \pm 0.27(2)$ & $9.00 \pm 0.30$ & $7.08 \pm 0.30(1)$ & $7.39 \pm 0.28$ \\
\hline NGC 346-012 & 24200 & 3.20 & 9 & 28 & $7.45 \pm 0.14(1)$ & $6.93 \pm 0.12(2)$ & $8.13 \pm 0.06(27)$ & $6.70 \pm 0.13$ & $6.82 \pm 0.16(3)$ \\
\hline NGC 346-021 & 25150 & 3.50 & 3 & 15 & $7.52 \pm 0.15(1)$ & $6.84 \pm 0.10$ & $8.16 \pm 0.12(28)$ & $6.76 \pm 0.14$ & $6.78 \pm 0.21(3)$ \\
\hline NGC 346-026 & 31000 & 3.70 & 5 & 75 & - & $7.79 \pm 0.22(1)$ & $7.94 \pm 0.17$ (3) & $6.65 \pm 0.10$ & $6.79 \pm 0.26(3)$ \\
\hline NGC 346-027 & 31000 & 4.05 & 8 & 220 & - & $<7.71 \pm 0.20(1)$ & $8.18 \pm 0.18$ & $7.00 \pm 0.09$ & $6.81 \pm 0.25$ \\
\hline NGC 346-029 & 32150 & 4.10 & - & 25 & $7.33 \pm 0.26(1)$ & $<6.99 \pm 0.25(1)$ & $8.02 \pm 0.15(18)$ & $6.69 \pm 0.21$ & $6.69 \pm 0.20(3)$ \\
\hline NGC 346-032 & 29000 & 4.40 & - & 125 & $7.44 \pm 0.19$ & $<6.88 \pm 0.18(1)$ & $7.87 \pm 0.09(13)$ & - & $6.58 \pm 0.19(3)$ \\
\hline NGC 346-037 & 18800 & 3.20 & 3 & 35 & $7.05 \pm 0.10(2)$ & $7.55 \pm 0.21$ & $7.94 \pm 0.28$ & $6.62 \pm 0.18$ & $6.79 \pm 0.28$ \\
\hline NGC 346-039 & 25800 & 3.60 & 3 & 20 & $7.54 \pm 0.14$ & $<6.59 \pm 0.15(1)$ & $8.24 \pm 0.10(28)$ & $6.73 \pm 0.14$ & $6.79 \pm 0.22(3)$ \\
\hline NGC 346-040 & 30600 & 4.00 & - & 20 & $7.28 \pm 0.21(1)$ & $<6.88 \pm 0.2$ & $7.95 \pm 0.11(17)$ & $6.39 \pm 0.19$ & $6.56 \pm 0.18$ \\
\hline NGC 346-043 & 33000 & 4.25 & - & 10 & $7.37 \pm 0.30(1)$ & $<6.75 \pm 0.30(1)$ & $8.00 \pm 0.14(19)$ & $6.83 \pm 0.27(1)$ & $6.69 \pm 0.19(3)$ \\
\hline
\end{tabular}


Table 2. continued.

\begin{tabular}{|c|c|c|c|c|c|c|c|c|c|}
\hline Star & $\begin{array}{l}T_{\text {eff }} \\
(\mathrm{K})\end{array}$ & $\begin{array}{l}\log g \\
\text { (dex) }\end{array}$ & $\begin{array}{l}\xi_{\mathrm{vt}} \\
\mathrm{m} \mathrm{s}^{-1}\end{array}$ & $\begin{array}{c}v \sin i \\
\left(\mathrm{~km} \mathrm{~s}^{-1}\right)\end{array}$ & $\begin{array}{l}\text { Carbon } \\
\text { (dex) }\end{array}$ & $\begin{array}{c}\text { Nitrogen } \\
(\text { dex })\end{array}$ & $\begin{array}{c}\text { Oxygen } \\
(\text { dex })\end{array}$ & $\begin{array}{c}\text { Magnesium } \\
\text { (dex) }\end{array}$ & $\begin{array}{c}\text { Silicon } \\
\text { (dex) }\end{array}$ \\
\hline NGC 346-044 & 23000 & 3.50 & 4 & 40 & $7.50 \pm 0.13(1)$ & $<6.94 \pm 0.10(2)$ & $8.13 \pm 0.18(21)$ & $6.66 \pm 0.14(1)$ & $6.76 \pm 0.25(3)$ \\
\hline NGC 346-047 & 19850 & 3.25 & 2 & 63 & $7.18 \pm 0.06(1)$ & $7.41 \pm 0.24(6)$ & $7.80 \pm 0.31(7)$ & $6.74 \pm 0.17$ & $6.77 \pm 0.35$ \\
\hline NGC 346-053 & 29500 & 3.75 & 0 & 170 & $7.77 \pm 0.29(1)$ & $<7.44 \pm 0.29(1)$ & $8.39 \pm 0.19(8)$ & $6.84 \pm 0.26(1)$ & $6.80 \pm 0.36(1)$ \\
\hline NGC 346-054 & 29000 & 4.30 & 0 & 23 & $7.29 \pm 0.19(1)$ & $<6.77 \pm 0.21(1)$ & $7.75 \pm 0.08(24)$ & $6.45 \pm 0.17(1)$ & $6.41 \pm 0.18$ \\
\hline NGC 346-055 & 29500 & 4.00 & 3 & 130 & $7.45 \pm 0.14$ (1) & $<7.25 \pm 0.16(1)$ & $8.10 \pm 0.12(7)$ & $6.57 \pm 0.11$ & $6.80 \pm 0.24$ \\
\hline NGC 346-056 & 31000 & 3.80 & 1 & 15 & $7.15 \pm 0.23(1)$ & $7.40 \pm 0.14$ & $8.00 \pm 0.18$ & $6.81 \pm 0.19$ & $6.77 \pm 0.21$ \\
\hline NGC 346-057 & 19850 & 3.35 & 4 & 73 & $7.16 \pm 0.21(1)$ & $<6.71 \pm 0.27(1)$ & $8.19 \pm 0.38$ & $6.53 \pm 0.24(1)$ & $6.81 \pm 0.35$ \\
\hline NGC 346-058 & 29500 & 4.25 & 6 & 180 & - & $<7.56 \pm 0.26(1)$ & $8.08 \pm 0.15(3)$ & - & $6.80 \pm 0.24$ \\
\hline NGC 346-062 & 29750 & 4.00 & 6 & 25 & $7.35 \pm 0.18$ (1) & $7.28 \pm 0.10$ & $7.91 \pm 0.07(21)$ & $6.75 \pm 0.17(1)$ & $6.82 \pm 0.17$ \\
\hline NGC 346-070 & 30500 & 4.15 & 3 & 109 & - & $<7.50 \pm 0.23(1)$ & $8.00 \pm 0.12(9)$ & $6.77 \pm 0.18$ & $6.80 \pm 0.25(2)$ \\
\hline NGC 346-074 & 16300 & 3.20 & 5 & 52 & $6.92 \pm 0.33(1)$ & $7.50 \pm 0.38$ & - & $6.53 \pm 0.27(1)$ & $6.82 \pm 0.46$ \\
\hline NGC 346-075 & 27700 & 4.30 & 2 & 10 & $7.56 \pm 0.15(1)$ & $<6.48 \pm 0.14(1)$ & $8.06 \pm 0.08(27)$ & $6.88 \pm 0.15$ & $6.81 \pm 0.18$ \\
\hline NGC 346-079 & 29500 & 4.20 & 2 & 293 & - & $<7.88 \pm 0.25(1)$ & - & - & $6.83 \pm 0.32(1)$ \\
\hline NGC 346-080 & 27300 & 4.25 & 1 & 216 & - & $<7.85 \pm 0.18(1)$ & $8.25 \pm 0.17(5)$ & - & $6.76 \pm 0$. \\
\hline NGC 346-081 & 21200 & 3.50 & 3 & 255 & $7.02 \pm 0.20(1)$ & $<7.52 \pm 0.31(1)$ & $7.91 \pm 0.37$ & - & $6.78 \pm 0.40$ \\
\hline NGC 346-082 & 21200 & 3.70 & 0 & 168 & $7.30 \pm 0.20(1)$ & $<7.55 \pm 0.32(1)$ & $7.76 \pm 0.37(1)$ & $6.56 \pm 0.24$ & $6.80 \pm 0.38(2)$ \\
\hline NGC 346-083 & 27300 & 4.05 & 1 & 207 & - & $<7.73 \pm 0.25(1)$ & - & - & $6.80 \pm 0.31(1)$ \\
\hline NGC 346-084 & 27300 & 4.25 & - & 105 & $7.48 \pm 0.20(1)$ & $<7.06 \pm 0.19(1)$ & $7.75 \pm 0.13(5)$ & - & $6.57 \pm 0.22(2)$ \\
\hline NGC 346-088 & 27530 & 4.10 & 5 & 84 & $7.78 \pm 0.19(1)$ & $<6.94 \pm 0.15(1)$ & $7.99 \pm 0.09(15)$ & $6.85 \pm 0.15$ & $6.83 \pm 0.21$ \\
\hline NGC 346-092 & 27300 & 3.95 & 4 & 234 & $7.72 \pm 0.24(1)$ & $<7.52 \pm 0.22(1)$ & $8.09 \pm 0.13(6)$ & - & $6.80 \pm 0.33$ \\
\hline NGC 346-094 & 28500 & 4.00 & 6 & 40 & $7.68 \pm 0.16(1)$ & $7.34 \pm 0.17$ & \pm 0.11 & $\pm 0.15(1)$ & $6.80 \pm 0.19$ \\
\hline NGC 346-098 & 26100 & 4.05 & 1 & 56 & $7.63 \pm 0.20(1)$ & $6.94 \pm 0.19$ & $7.80 \pm 0.10(19)$ & $\pm 0.20(1)$ & $6.77 \pm 0.19$ \\
\hline NGC 346-099 & 18000 & 3.40 & 7 & 94 & $7.13 \pm 0.26(1)$ & $<7.14 \pm 0.33(1)$ & - & $6.47 \pm 0.25$ & $6.79 \pm 0.43(1)$ \\
\hline NGC 346-100 & 26100 & 4.30 & - & 183 & $7.56 \pm 0.22(1)$ & $<7.41 \pm 0.22(1)$ & $8.02 \pm 0.21(2)$ & - & $6.57 \pm 0.29$ \\
\hline NGC 346-101 & 27300 & 4.25 & - & 29 & $7.33 \pm 0.15(1)$ & $6.63 \pm 0.14$ & $7.50 \pm 0.08$ & $6.49 \pm 0.13$ & $6.43 \pm 0.15$ \\
\hline NGC 346-102 & 17700 & 3.70 & 3 & 30 & $6.88 \pm 0.2$ & $<6.97 \pm 0.34(1)$ & $8.41 \pm 0.47(1)$ & - & $6.81 \pm 0.39(2)$ \\
\hline NGC 346-103 & 29500 & 4.00 & 1 & 10 & $7.23 \pm 0.16(1)$ & $7.58 \pm 0$ & & $6.82 \pm 0$ & (3) \\
\hline NGC 346 & 32500 & 4.35 & - & 17 & - & $<7.20 \pm 0$. & $7.73 \pm($ & $6.51 \pm 0.24$ & $6.53 \pm$ \\
\hline NGC 346 & 27500 & 4.20 & - & 142 & - & $<7.51 \pm 0.26(1)$ & $8.09 \pm 0$ & - & $6.50 \pm 0$. \\
\hline NGC 346 & 26100 & 3.95 & 5 & 167 & $7.59 \pm 0.15(1)$ & $<7.42 \pm 0.16(1)$ & $7.96 \pm 0.1$ & $6.78 \pm 0.14(1)$ & $6.76 \pm 0.23(2)$ \\
\hline NGC 346-109 & 26100 & 4.25 & 5 & 123 & $7.70 \pm 0.1$ & $<7.09 \pm 0.11(1)$ & $7.83 \pm 0.12$ & - & $6.77 \pm 0.18$ \\
\hline NGC 346-114 & 27300 & 3.90 & 5 & 287 & - & $<7.80 \pm 0.25(1)$ & - & - & $6.77 \pm 0.34(1)$ \\
\hline NGC 346-116 & 28250 & 4.10 & 0 & 15 & $7.43 \pm 0.17(1)$ & $6.93 \pm 0.17$ & $8.13 \pm 0.09(24)$ & $6.70 \pm 0.16(1)$ & $6.81 \pm 0.20(3)$ \\
\hline NGC 330-002 & 14590 & 2.15 & 16 & 24 & $6.91 \pm 0.22(1)$ & $7.60 \pm 0.27(6)$ & $7.98 \pm 0.38$ & $6.60 \pm 0.19$ & $6.82 \pm 0.35$ \\
\hline NGC 330-003 & 17210 & 2.25 & 20 & 36 & $7.14 \pm 0.15(1)$ & $7.69 \pm 0.14(6)$ & $8.07 \pm 0.2$ & $6.79 \pm 0.19$ & $6.81 \pm 0.25$ \\
\hline NGC 330-004 & 17000 & 2.30 & 16 & 41 & $6.72 \pm 0.06(1)$ & $7.84 \pm 0.12(6)$ & $7.86 \pm 0.22(15)$ & $6.73 \pm 0.13$ & $6.82 \pm 0.27$ \\
\hline NGC 330-005 & 13700 & 2.25 & 8 & 20 & $6.59 \pm 0.28$ (1) & $7.45 \pm 0.29(3)$ & $7.82 \pm 0.36(2)$ & $6.58 \pm 0.25(1)$ & $6.81 \pm 0.32(3)$ \\
\hline NGC 330-009 & 13940 & 2.45 & 6 & 28 & $6.87 \pm 0.32(1)$ & $7.20 \pm 0.33(2)$ & $8.30 \pm 0.43(3)$ & $6.66 \pm 0.28$ & $6.81 \pm 0.38(2)$ \\
\hline & 14820 & 2.60 & 4 & 15 & $99 \pm 0.2$ & $7.14 \pm 0$ & $7.71 \pm 0$ & $6.63 \pm 0$ & $6.84 \pm$ \\
\hline 014 & 20130 & 2.75 & 18 & 78 & $7.02 \pm 0.1$ & $7.52 \pm 0.10(5)$ & (20) & 6.72 & $6.83 \pm$ \\
\hline 016 & 14220 & 2.60 & 5 & 45 & (1) & $7.18 \pm 0.35$ & $(2)$ & 6.69 & (2) \\
\hline 17 & 22000 & 3.35 & - & 21 & $7.30 \pm 0.1$ & $7.15 \pm 0.08(6)$ & $7.64 \pm 0$. & $6.47 \pm 0$ & $7(3)$ \\
\hline C $330-018$ & 18000 & 2.95 & 5 & 48 & $7.10 \pm 0.15(1)$ & $7.24 \pm 0.20(2)$ & $8.05 \pm 0.2$ & $6.61 \pm 0$ & $6.80 \pm 0.26$ \\
\hline C $330-020$ & 16720 & 2.85 & 5 & 42 & $6.92 \pm 0.22(1)$ & $7.01 \pm 0.23$ & $7.80 \pm 0.29$ (4) & $6.64 \pm 0.2$ & $6.83 \pm 0.28$ \\
\hline NGC 330-021 & 30500 & 3.70 & 12 & 204 & - & $<7.64 \pm 0.27(1)$ & - & $6.91 \pm 0.21$ & $6.93 \pm 0.31$ \\
\hline NGC 330-022 & 18860 & 3.00 & 1 & 24 & $7.11 \pm 0.07(1)$ & $7.28 \pm 0.17(6)$ & $7.76 \pm 0.27(8)$ & $6.85 \pm 0.15$ & $6.83 \pm 0.31(3)$ \\
\hline NGC 330-024 & 14000 & 2.70 & 4 & 46 & $7.09 \pm 0.46(1)$ & $<7.53 \pm 0.46(1)$ & - & $6.52 \pm 0.30(1)$ & - \\
\hline NGC 330-026 & 22500 & 3.40 & - & 73 & $7.57 \pm 0.17$ (1) & $7.23 \pm 0.15$ & $7.74 \pm 0.20(6)$ & $7.02 \pm 0.19$ & $6.64 \pm 0.25$ \\
\hline NGC 330-027 & 22040 & 3.20 & 7 & 80 & $7.36 \pm 0.17$ (1) & $7.40 \pm 0.15(2)$ & $8.27 \pm 0.22(8)$ & $6.44 \pm 0.17$ & $6.81 \pm 0.22(3)$ \\
\hline NGC 330-028 & 27300 & 3.75 & 7 & 76 & $7.84 \pm 0.24(1)$ & $7.51 \pm 0.20(2)$ & $7.99 \pm 0.07(21)$ & $6.86 \pm 0.21$ & $6.80 \pm 0.24$ \\
\hline NGC 330-032 & 29700 & 4.15 & 0 & 17 & $7.37 \pm 0.21(1)$ & $7.37 \pm 0.09$ (3) & $7.90 \pm 0.07(22)$ & $6.80 \pm 0.20$ & $6.77 \pm 0.19$ \\
\hline NGC 330-033 & 26100 & 3.90 & 0 & 105 & $7.74 \pm 0.16(1)$ & $7.07 \pm 0.14$ & $7.66 \pm 0.06(3)$ & $7.26 \pm 0.16(1)$ & $6.78 \pm 0.25$ \\
\hline $330-035$ & 19000 & 3.20 & - & 40 & $6.94 \pm 0.21$ & $7.26 \pm 0.31(2)$ & $8.04 \pm 0.44$ (2) & $6.68 \pm 0$ & $6.70 \pm 0.39$ \\
\hline C $330-036$ & 21200 & 3.25 & 6 & 39 & $7.18 \pm 0.16(1)$ & $7.17 \pm 0.18$ & $7.93 \pm 0.27(18)$ & $6.45 \pm 0.19$ & $6.79 \pm 0.31$ \\
\hline $330-038$ & 27300 & 3.75 & 2 & 150 & $7.67 \pm 0.20(1)$ & $<7.16 \pm 0.20(1)$ & $8.27 \pm 0.11(8)$ & $6.81 \pm 0.18$ & $6.78 \pm 0.27(2)$ \\
\hline $330-039$ & 33000 & 4.25 & 9 & 120 & - & $<7.61 \pm 0.31(1)$ & - & - & $6.81 \pm 0.31$ \\
\hline C $330-040$ & 21200 & 3.25 & 7 & 106 & $7.39 \pm 0.21(1)$ & $<7.12 \pm 0.23(1)$ & $8.27 \pm 0.35$ & $6.81 \pm 0.24(1)$ & $6.88 \pm 0.36(2)$ \\
\hline C $330-041$ & 32000 & 4.15 & 4 & 127 & $7.86 \pm 0.24$ (1) & $7.73 \pm 0.24$ & $8.30 \pm 0.19(7)$ & - & $6.77 \pm 0.33$ \\
\hline C $330-042$ & 25450 & 3.75 & 1 & 27 & $7.33 \pm 0.17(1)$ & $7.18 \pm 0.04(6)$ & $7.73 \pm 0.09(19)$ & $6.88 \pm 0.17(1)$ & $6.82 \pm 0.22$ \\
\hline $330-045$ & 18450 & 3.25 & 8 & 133 & $6.96 \pm 0.22(1)$ & $<7.28 \pm 0.33(1)$ & $8.29 \pm 0.42(1)$ & - & $6.85 \pm 0.42(1)$ \\
\hline 330 & 700 & 4.05 & 0 & 28 & $7.47 \pm 0.14(1)$ & $6.76 \pm 0.11$ & $8.07 \pm 0.10(24)$ & $6.57 \pm 0.14$ & $6.83 \pm 0.23(3)$ \\
\hline & 000 & 4.00 & 5 & 73 & $7.42 \pm 0.22(1)$ & $<7.10 \pm 0.24(1)$ & $8.12 \pm 0.13(18)$ & - & $6.81 \pm 0.24$ \\
\hline $330-049$ & 35000 & 4.15 & - & 40 & - & $6.69 \pm 1.18$ & - & - & - \\
\hline C $330-051$ & 26100 & 4.05 & 5 & 273 & - & $<7.20 \pm 0.22(1)$ & $8.33 \pm 0.28$ (1) & - & $6.80 \pm 0.32(1)$ \\
\hline NGC 330-053 & 29650 & 4.25 & 8 & 82 & $7.54 \pm 0.17$ (1) & $<7.04 \pm 0.20(1)$ & $8.04 \pm 0.08$ & $6.97 \pm 0.16(1)$ & $6.84 \pm 0.25$ \\
\hline NGC 330-055 & 31000 & 4.10 & - & 219 & - & $8.06 \pm 0.22(1)$ & - & - & - \\
\hline
\end{tabular}


Table 2. continued.

\begin{tabular}{|c|c|c|c|c|c|c|c|c|c|}
\hline Star & $\begin{array}{l}T_{\text {eff }} \\
(\mathrm{K})\end{array}$ & $\begin{array}{l}\log g \\
(\operatorname{dex})\end{array}$ & $\begin{array}{c}\xi_{\mathrm{vt}} \\
\left(\mathrm{km} \mathrm{s}^{-1}\right)\end{array}$ & $\begin{array}{c}v \sin i \\
\left(\mathrm{~km} \mathrm{~s}^{-1}\right)\end{array}$ & $\begin{array}{l}\text { Carbon } \\
\text { (dex) }\end{array}$ & $\begin{array}{l}\text { Nitrogen } \\
(\text { dex })\end{array}$ & $\begin{array}{l}\text { Oxygen } \\
\text { (dex) }\end{array}$ & $\begin{array}{l}\text { Magnesium } \\
\text { (dex) }\end{array}$ & $\begin{array}{c}\text { Silicon } \\
\text { (dex) }\end{array}$ \\
\hline NGC 330-056 & 21200 & 3.50 & 8 & 108 & $7.25 \pm 0.13(1)$ & $<6.93 \pm 0.18$ & $8.52 \pm 0.34(9)$ & $6.55 \pm 0.17(1)$ & $6.81 \pm 0.30(3)$ \\
\hline NGC 330-057 & 29000 & 4.15 & 5 & 104 & - & $<7.48 \pm 0.29(1)$ & $8.01 \pm 0.18$ & $6.96 \pm 0.26(1)$ & - \\
\hline NGC 330-059 & 19000 & 3.30 & 8 & 123 & $7.17 \pm 0.21$ & $7.27 \pm 0.32$ & - & - & $6.80 \pm 0.42(1)$ \\
\hline NGC 330-066 & 18500 & 3.40 & 5 & 126 & $7.08 \pm 0.24(1)$ & $<7.77 \pm 0.34(1)$ & - & - & - \\
\hline NGC 330-067 & 19850 & 3.40 & 9 & 65 & $7.15 \pm 0.28$ & $7.28 \pm 0.29(2)$ & $8.17 \pm 0.36(4)$ & - & $6.78 \pm 0.38(2)$ \\
\hline NGC 330-069 & 19000 & 3.40 & 5 & 193 & $7.30 \pm 0.23(1)$ & $<7.91 \pm 0.33(1)$ & - & $6.88 \pm 0.26(1)$ & - \\
\hline NGC 330-072 & 29650 & 4.15 & 5 & 99 & $7.67 \pm 0.27(1)$ & $<7.30 \pm 0.29(1)$ & $8.09 \pm 0.13(9)$ & $6.99 \pm 0.26(1)$ & $6.81 \pm 0.21(3)$ \\
\hline NGC 330-074 & 32020 & 4.20 & 4 & 29 & $7.42 \pm 0.21(1)$ & $7.45 \pm 0.17(2)$ & $7.87 \pm 0.13$ & $6.81 \pm 0.18$ & $6.83 \pm 0.19$ \\
\hline NGC 330-079 & 19500 & 3.40 & 5 & 146 & $7.29 \pm 0.21(1)$ & $<7.69 \pm 0.35(1)$ & - & - & - \\
\hline NGC 330-083 & 18000 & 3.25 & 5 & 140 & $7.09 \pm 0.26(1)$ & $<7.66 \pm 0.37(1)$ & - & - & - \\
\hline NGC 330-086 & 19850 & 3.50 & 7 & 89 & $7.06 \pm 0.20(1)$ & $<7.33 \pm 0.31(1)$ & $7.88 \pm 0.37(1)$ & - & $6.77 \pm 0.39(1)$ \\
\hline NGC 330-095 & 18450 & 3.45 & 7 & 20 & $7.25 \pm 0.2$ & $<6.95 \pm$ & $8.42 \pm 0.46$ & $6.33 \pm 0.25(1)$ & $6.81 \pm 0$ \\
\hline 330-097 & 27300 & 3.90 & 7 & 154 & $7.76 \pm 0.1$ & 7.60 & $8.24 \pm 0$ & - & $6.82 \pm 0$ \\
\hline NGC 330-101 & 19850 & 3.50 & 3 & 48 & $7.27 \pm 0.09(1)$ & $7.07 \pm 0.2$ & $8.35 \pm 0.33(4)$ & $6.61 \pm 0.16(1)$ & $6.83 \pm 0.35(2)$ \\
\hline NGC 330-107 & 16500 & 3.25 & 6 & 34 & $7.16 \pm 0.33(1)$ & $<7.42 \pm 0.40(1)$ & - & $6.64 \pm 0.2$ & $6.78 \pm 0.46(1)$ \\
\hline NGC 330-108 & 14500 & 3.30 & 5 & 102 & - & - & - & $6.77 \pm 0.32(1)$ & - \\
\hline NGC 330-109 & 18450 & 3.20 & 5 & 213 & - & - & - & $6.74 \pm 0.26(1)$ & - \\
\hline NGC 330-110 & 21200 & 3.40 & 11 & 110 & $7.10 \pm 0.17$ & $7.23 \pm 0.19(2)$ & $8.32 \pm 0.32(9)$ & $6.55 \pm 0.20$ & $6.82 \pm 0.32(2)$ \\
\hline NGC 330-114 & 23800 & 3.90 & 4 & 17 & $7.53 \pm 0.19(1)$ & $7.32 \pm 0.15(3)$ & $8.00 \pm 0.17(18)$ & $6.97 \pm 0.20$ & $6.83 \pm 0.21(3)$ \\
\hline NGC 330-116 & 19500 & 3.55 & 5 & 4 & $7.37 \pm 0.19(1)$ & $7.05 \pm 0.27$ & $8.42 \pm 0.41(3)$ & $6.70 \pm 0.21$ & $6.82 \pm 0.38$ \\
\hline NGC 330-120 & 18500 & 3.40 & 5 & 137 & $6.90 \pm 0.22(1)$ & $<7.85 \pm 0.33(1)$ & - & - & - \\
\hline NGC 330-123 & 35000 & 4.25 & - & 26 & - & - & - & $6.71 \pm 0.21(1)$ & - \\
\hline NGC 330-124 & 30980 & 4.25 & 2 & 95 & $7.71 \pm 0.20(1)$ & $7.36 \pm 0.20(1)$ & $7.75 \pm 0.14(3)$ & $6.75 \pm 0.17(1)$ & $6.84 \pm 0.19$ \\
\hline NGC 330-125 & 21200 & 3.50 & 6 & 86 & $7.14 \pm 0.28$ & $7.29 \pm 0.2$ & $8.27 \pm 0.3$ & $6.35 \pm 0$ & $6.81 \pm 0.33(3)$ \\
\hline N11-001 & 18750 & 2.50 & 14 & 52 & $7.32 \pm 0.20(1)$ & $8.20 \pm 0.20$ & $8.23 \pm 0.2$ & & \\
\hline $\mathrm{N} 11-002$ & 15800 & 2.10 & 18 & 53 & $7.38 \pm 0.2$ & $8.00 \pm 0$ & & & \\
\hline N11 & 23200 & 2.75 & 13 & 54 & 7.6 & 7.09 & & & \\
\hline N11-008 & 25450 & 3.00 & 16 & 43 & $7.76 \pm 0.20(1)$ & $7.84 \pm 0.1$ & $8.25 \pm 0$. & $7.12 \pm 0$ & $7.19 \pm 0.16(3)$ \\
\hline N11-009 & 15000 & 2.15 & 17 & 30 & $7.41 \pm 0.24(1)$ & $7.74 \pm 0$ & $8.38 \pm 0$ & $6.95 \pm 0$. & $7.17 \pm 0.32(3)$ \\
\hline N11-012 & 20500 & 2.55 & 13 & 40 & $7.35 \pm 0.24(1)$ & $7.71 \pm 0.08$ & $8.42 \pm 0.15$ (19) & $7.02 \pm 0.24(1)$ & $7.15 \pm 0.24$ \\
\hline N11-014 & 19100 & 2.55 & 12 & 30 & $7.59 \pm 0.18$ & $7.89 \pm 0.15$ & $8.27 \pm 0.16(26)$ & $7.16 \pm 0.20$ & $7.17 \pm 0.28$ \\
\hline N11-015 & 23600 & 2.95 & 11 & 58 & $7.75 \pm 0.28(1)$ & $7.14 \pm 0.28$ & $8.36 \pm 0.12(20)$ & $7.01 \pm 0.27$ & $7.21 \pm 0.25$ \\
\hline N11-016 & 21700 & 2.75 & 12 & 54 & $7.76 \pm 0.22(1)$ & $7.90 \pm 0.10$ & $8.31 \pm 0.16(17)$ & $7.27 \pm 0.21$ & $7.17 \pm 0.27$ \\
\hline N11-017 & 16500 & 2.30 & 16 & 25 & $7.38 \pm 0.18$ (1) & $7.89 \pm 0.22(6)$ & $8.33 \pm 0.28(23)$ & $7.00 \pm 0.19$ & $7.19 \pm 0.30(3)$ \\
\hline N11-023 & 24000 & 2.90 & 13 & 66 & $7.76 \pm 0.18(1)$ & $7.16 \pm 0.18$ & $8.41 \pm 0.14(18)$ & $7.00 \pm 0.17$ & $7.17 \pm 0.24(3)$ \\
\hline N11-024 & 21600 & 2.80 & 12 & 30 & $7.65 \pm 0.19(1)$ & $7.85 \pm 0.10$ & $8.32 \pm 0.16(22)$ & $7.14 \pm 0.19$ & $7.15 \pm 0.27(3)$ \\
\hline N11-029 & 3750 & 3.30 & 15 & 40 & $7.81 \pm 0.33(1)$ & $7.10 \pm 0.35$ & $8.28 \pm 0.1$ & $6.93 \pm 0.30$ & $7.17 \pm 0.24$ \\
\hline N11- & 500 & 3.25 & 12 & 203 & $8.10 \pm 0.23(1)$ & $7.12 \pm 0.15(2)$ & $8.39 \pm 0$. & \pm 0.2 & $7.51 \pm 0.29$ \\
\hline & 750 & 3.10 & 11 & 30 & $7.66 \pm 0$. & 7.76 & 8.3 & $7.03=$ & 7.17 \\
\hline 37 & 100 & 3.25 & 12 & 100 & (1) & $<7.17 \pm$ & $8.18 \pm 0$ & $7.01 \pm$ & (3) \\
\hline & 1700 & 3.00 & 9 & 157 & $8.02 \pm 0.2$ & $6.91 \pm 0$ & $8.09 \pm 0$ & $7.40 \pm$ & $7.20 \pm 0$ \\
\hline & 29000 & 3.60 & 5 & 30 & $7.75 \pm 0.24(1)$ & $6.92 \pm 0.2$ & $8.34 \pm 0$ & $7.00 \pm 0$ & $7.21 \pm 0$. \\
\hline & 33500 & 4.25 & - & 205 & $7.90 \pm 0.2$ & $<7.71 \pm 0$ & $8.43 \pm 0$ & $7.10 \pm 0$. & $7.08 \pm 0.35(1)$ \\
\hline & 29200 & 3.65 & 8 & 55 & $7.89 \pm 0.2$ & $<6.88 \pm 0$ & $8.24 \pm 0.1$ & $7.00 \pm 0$ & $7.20 \pm 0.19$ \\
\hline N11-054 & 23500 & 3.05 & 10 & 45 & $7.83 \pm 0.19(1)$ & $6.86 \pm 0.12(2)$ & $8.45 \pm 0.12(26)$ & $6.98 \pm 0.18$ & $7.16 \pm 0.26(3)$ \\
\hline N11-059 & 34000 & 3.85 & 12 & 103 & - & - & $8.55 \pm 0.18(6)$ & $7.20 \pm 0.35$ & $7.47 \pm 0.35$ \\
\hline $\mathrm{N} 11-062$ & 30400 & 4.05 & 5 & 25 & $7.61 \pm 0.22(1)$ & $7.16 \pm 0.16(2)$ & $8.25 \pm 0.11(26)$ & $6.99 \pm 0.20$ & $7.16 \pm 0.21(3)$ \\
\hline N11-069 & 24300 & 3.30 & 11 & 80 & $7.92 \pm 0.23(1)$ & $6.94 \pm 0.22(1)$ & $8.44 \pm 0.12(26)$ & $7.06 \pm 0.22(1)$ & $7.18 \pm 0.24$ \\
\hline N11-070 & 19500 & 3.30 & 0 & 62 & $7.48 \pm 0.15(1)$ & $6.85 \pm 0.22(2)$ & $8.24 \pm 0.40(14)$ & $6.86 \pm 0.21$ & $7.24 \pm 0.42(3)$ \\
\hline N11-072 & 28800 & 3.75 & 5 & 15 & $7.82 \pm 0.20(1)$ & $7.38 \pm 0.06$ & $8.36 \pm 0.12(25)$ & $7.12 \pm 0.20$ & $7.21 \pm 0.23$ \\
\hline N11-075 & 21800 & 3.35 & 5 & 25 & $7.74 \pm 0.13(1)$ & $8.00 \pm 0.21$ & $8.18 \pm 0.20(25)$ & $7.16 \pm 0.16(1)$ & $7.17 \pm 0.32$ \\
\hline N11-077 & 21500 & 3.30 & 3 & 117 & $7.66 \pm 0.19(1)$ & $6.96 \pm 0.17(2)$ & $8.44 \pm 0.33(20)$ & $6.94 \pm 0.22(1)$ & $7.21 \pm 0.37$ \\
\hline N11-079 & 32500 & 4.25 & - & 38 & $7.74 \pm 0.28$ & $7.11 \pm 0.23(2)$ & $8.17 \pm 0.16(25)$ & $6.96 \pm 0.25$ & $6.99 \pm 0.22(3)$ \\
\hline & 300 & 4.15 & - & 20 & $7.85 \pm 0.20$ & $6.86 \pm 0.19$ & 8.3 & & $7.06 \pm 0.22$ \\
\hline & 9500 & 4.25 & - & 108 & $7.76 \pm 0.24(1)$ & $<6.89 \pm 0.24(1)$ & $8.24 \pm 0.11$ & $6.94 \pm 0.23$ & $6.91 \pm 0.23$ \\
\hline & 6800 & 4.25 & - & 75 & $7.36 \pm 0.1$ & $6.82 \pm 0.14(2)$ & $8.01 \pm 0$. & $6.79 \pm 0$ & $6.72 \pm 0.19$ \\
\hline & -150 & 3.45 & 8 & 240 & $7.59 \pm 0.1$ & $<6.96 \pm 0.1$ & & $6.93 \pm 0$ & $7.22 \pm 0.25(3)$ \\
\hline & 1700 & 3.20 & 5 & 117 & $7.88 \pm 0.20(1)$ & $6.91 \pm 0.12(2)$ & $8.05 \pm 0.26$ & $7.30 \pm 0$ & $7.20 \pm 0.37$ \\
\hline & 19500 & 3.30 & 1 & 73 & $7.60 \pm 0.19$ & $6.80 \pm 0.21(2)$ & $8.41 \pm 0.41(13)$ & $6.99 \pm 0.25$ & $7.16 \pm 0.41$ \\
\hline & 26800 & 3.85 & 2 & 267 & $7.63 \pm 0.24(1)$ & $7.46 \pm 0.24(1)$ & $8.31 \pm 0.13$ & $6.69 \pm 0.22(1)$ & $7.17 \pm 0.37(1)$ \\
\hline & 7500 & 2.95 & - & 52 & $7.31 \pm 0.28$ & $7.68 \pm 0.36(3)$ & $8.33 \pm 0.47(3)$ & $7.14 \pm 0.32$ & $7.03 \pm 0.45$ \\
\hline & 1700 & 3.45 & 2 & 45 & $7.71 \pm 0.21$ & $7.02 \pm 0.19(2)$ & $8.27 \pm 0.29(26)$ & $7.03 \pm 0.25$ & $7.21 \pm 0.35$ \\
\hline & 29700 & 4.15 & 4 & 30 & $7.65 \pm 0.23(1)$ & $7.62 \pm 0.17(2)$ & $8.30 \pm 0.10(26)$ & $7.10 \pm 0.22$ & $7.20 \pm 0.22(3)$ \\
\hline & 29800 & 3.95 & 8 & 70 & $7.94 \pm 0.21(1)$ & $<7.09 \pm 0.21(1)$ & $8.32 \pm 0.10(21)$ & $7.21 \pm 0.20$ & $7.16 \pm 0.16(3)$ \\
\hline $\mathrm{N} 11-102$ & 31000 & 4.20 & 0 & 218 & $7.78 \pm 0.22(1)$ & $<7.58 \pm 0.23(1)$ & $8.31 \pm 0.18$ & $7.25 \pm 0.18$ & $7.20 \pm 0.35$ \\
\hline N11-104 & 25700 & 3.75 & 1 & 153 & $7.96 \pm 0.24(1)$ & $<7.20 \pm 0.21(1)$ & $8.28 \pm 0.14(14)$ & $7.15 \pm 0.23$ & $7.25 \pm 0.28$ \\
\hline
\end{tabular}


Table 2. continued.

\begin{tabular}{|c|c|c|c|c|c|c|c|c|c|}
\hline Star & $\begin{array}{l}T_{\text {eff }} \\
(\mathrm{K})\end{array}$ & $\begin{array}{l}\log g \\
(\operatorname{dex})\end{array}$ & $\begin{array}{c}\xi_{\mathrm{vt}} \\
\left(\mathrm{km} \mathrm{s}^{-1}\right)\end{array}$ & $\begin{array}{c}v \sin i \\
\left(\mathrm{~km} \mathrm{~s}^{-1}\right)\end{array}$ & $\begin{array}{l}\text { Carbon } \\
\text { (dex) }\end{array}$ & $\begin{array}{l}\text { Nitrogen } \\
(\mathrm{dex})\end{array}$ & $\begin{array}{l}\text { Oxygen } \\
\text { (dex) }\end{array}$ & $\begin{array}{l}\text { Magnesium } \\
(\text { dex })\end{array}$ & $\begin{array}{c}\text { Silicon } \\
\text { (dex) }\end{array}$ \\
\hline N11-106 & 31200 & 4.00 & 5 & 25 & $7.66 \pm 0.26(1)$ & $7.13 \pm 0.26(1)$ & $8.35 \pm 0.13(22)$ & $7.19 \pm 0.24(1)$ & $7.19 \pm 0.21(3)$ \\
\hline N11-108 & 32150 & 4.10 & 4 & 25 & $7.83 \pm 0.26(1)$ & $7.21 \pm 0.28$ & $8.27 \pm 0.13(17)$ & $7.14 \pm 0.23$ & $7.17 \pm 0.18$ \\
\hline N11-109 & 25750 & 3.20 & 10 & 40 & $7.75 \pm 0.20$ & $7.24 \pm 0.20$ & $8.32 \pm 0.12(17)$ & $6.84 \pm 0.19$ & $7.18 \pm 0.23$ \\
\hline N11-110 & 23100 & 3.25 & 7 & 25 & $7.79 \pm 0.16(1)$ & $7.39 \pm 0.06(3)$ & $8.48 \pm 0.18(26)$ & $7.06 \pm 0.16$ & $7.23 \pm 0.28$ \\
\hline N11-111 & 22950 & 3.50 & 3 & 101 & $7.67 \pm 0.21$ & $6.93 \pm 0.13(2)$ & $8.64 \pm 0.30(23)$ & $6.95 \pm 0.22$ & $7.19 \pm 0.32(3)$ \\
\hline N11-114 & 32500 & 4.05 & 2 & 299 & - & $<7.92 \pm 0.27(1)$ & - & $7.19 \pm 0.21(1)$ & $7.20 \pm 0.32$ \\
\hline N11-115 & 24150 & 3.65 & 1 & 53 & $7.63 \pm 0.17(1)$ & $6.89 \pm 0.10$ & $8.56 \pm 0.23(25)$ & $6.85 \pm 0.18$ & $7.22 \pm 0.29$ \\
\hline N11-116 & 21700 & 3.30 & 3 & 160 & $7.74 \pm 0.15$ & $6.98 \pm 0.13(2)$ & $8.39 \pm 0.32(12)$ & $7.05 \pm 0.19$ & $7.16 \pm 0.35$ \\
\hline N11-117 & 26800 & 4.20 & 0 & 247 & $7.46 \pm 0.23(1)$ & $<7.06 \pm 0.22(1)$ & $8.34 \pm 0.19$ & $6.89 \pm 0.22(1)$ & $7.19 \pm 0.36$ \\
\hline & 25700 & 3.80 & 2 & 150 & $7.93 \pm 0.28$ & $7.39 \pm 0.10$ & $8.26 \pm 0.13(16)$ & $7.25 \pm 0.27$ & $7.22 \pm 0.26$ \\
\hline N11-120 & 31500 & 4.10 & 2 & 207 & $7.90 \pm 0.20(1)$ & $<7.56 \pm 0$ & $8.51 \pm 0.21(3)$ & $7.17 \pm$ & (1) \\
\hline & 27000 & 4.15 & - & 265 & $7.49 \pm 0.23$ & $<7.48 \pm$ & $8.56 \pm 0.29(1)$ & $6.84 \pm 0$ & (1) \\
\hline & 330 & 4.10 & 2 & 173 & - & $<7.72 \pm$ & $8.29 \pm 0.25$ & $7.27 \pm$ & (1) \\
\hline N11-124 & 28500 & 4.20 & - & 45 & $7.80 \pm 0.1$ & $7.25 \pm 0$ & $8.12 \pm 0.1$ & $6.97 \pm$ & $6.94 \pm$ \\
\hline NGC 2004-003 & 14450 & 2.10 & 15 & 33 & $7.48 \pm 0.2$ & $7.85 \pm 0$ & $8.45 \pm 0.4$ & $7.02 \pm$ & $22 \pm($ \\
\hline NGC 2004 & 17160 & 2.40 & 14 & 30 & $7.42 \pm 0.20(1)$ & $8.16 \pm 0$. & $8.29 \pm 0.26$ & $7.11 \pm$ & $20 \pm 0$ \\
\hline C 2004 & 21250 & 2.75 & 13 & 40 & $7.63 \pm 0.20$ & $7.73 \pm 0.07(7)$ & $8.41 \pm 0.17$ & $7.15 \pm 0$ & $7.19 \pm 0$ \\
\hline 2002 & 21270 & 2.87 & 12 & 40 & $7.62 \pm 0.19$ & $7.75 \pm 0.08$ (7) & $8.43 \pm 0.17(31)$ & $7.08 \pm 0.19$ & $7.19 \pm 0.28$ \\
\hline NGC 2004 & 21700 & 3.20 & 3 & 145 & $7.77 \pm 0.18$ & $6.85 \pm 0.12(2)$ & $8.30 \pm 0.27$ & $7.19 \pm 0.22$ & $7.19 \pm 0.38$ \\
\hline NGC 2004 & 17800 & 2.85 & 10 & 30 & $7.50 \pm 0.19$ & $7.52 \pm 0.18(7)$ & $8.24 \pm 0.26(23)$ & $7.06 \pm 0.20$ & $7.20 \pm 0.30$ \\
\hline NGC 2004 & 22950 & 3.15 & 12 & 165 & $8.05 \pm 0.26(1)$ & $6.95 \pm 0.15(2)$ & $8.32 \pm 0.16$ & $7.25 \pm 0$ & $25(3)$ \\
\hline & 22950 & 3.20 & 9 & 145 & $8.14 \pm 0.20(1)$ & $7.46 \pm 0.08(3)$ & $8.22 \pm 0.17(8)$ & $7.32 \pm 0.18$ & $7.22 \pm 0.23(3)$ \\
\hline NGC 2004 & 21450 & 3.00 & 14 & 40 & $7.74 \pm 0.19$ & $7.20 \pm 0.09(6)$ & $8.47 \pm 0.18(29)$ & $7.07 \pm 0.19$ & $7.18 \pm 0.26$ \\
\hline $\mathrm{N}$ & 21780 & 3.15 & 11 & 30 & $7.53 \pm 0.16(1)$ & $7.68 \pm 0.09(7)$ & $8.55 \pm 0.19(32)$ & $6.94 \pm 0.17$ & \\
\hline & 22950 & 3.05 & 15 & 240 & $7.18 \pm 0.2$ & $8.09 \pm$ & $8.22 \pm 0.16(6)$ & $7.22 \pm 0$ & $7.17 \pm 0$ \\
\hline $\mathrm{NC}$ & & 3.65 & 0 & 20 & & $7.01=$ & & & \\
\hline NG & 23100 & 3.50 & 1 & 30 & $7.73 \pm 0$. & $6.81 \pm$ & $8.29 \pm$ & $7.03 \pm$ & $1.21 \pm$ \\
\hline $\mathrm{NO}$ & 29000 & 3.75 & 8 & 123 & $7.92 \pm 0$ & $7.43 \pm$ & $8.45 \pm$ & $7.16 \pm$ & $7.18 \pm$ \\
\hline 2004 & 21700 & 3.35 & 4 & 65 & $7.81 \pm 0$ & $6.84 \pm$ & $8.19 \pm 0$ & $7.17 \pm$ & $7.16 \pm$ \\
\hline NGC 2004 & 21700 & 3.40 & 5 & 110 & $7.73 \pm 0.16(1)$ & $6.90 \pm 0$. & $8.32 \pm 0.31(14)$ & $7.07 \pm 0.21$ & $7.15 \pm 0.35$ \\
\hline NGC 2004-036 & 22870 & 3.35 & 7 & 42 & $7.80 \pm 0.17$ & $7.38 \pm 0.06(6)$ & $8.48 \pm 0.18(28)$ & $6.93 \pm 0.16$ & $7.21 \pm 0.28$ \\
\hline NGC 2004-041 & 20450 & 3.30 & 2 & 101 & $7.67 \pm 0.17$ & $6.89 \pm 0.18$ (2) & $8.34 \pm 0.34(13)$ & $7.01 \pm 0.24$ & $7.20 \pm 0.38$ \\
\hline NGC 2004 & 20980 & 3.45 & 2 & 42 & $7.80 \pm 0.16(1)$ & $6.90 \pm 0.14(2)$ & $8.16 \pm 0.22(16)$ & $7.14 \pm 0.21$ & $7.19 \pm 0.30(3)$ \\
\hline NGC 2004 & 22950 & 3.50 & 7 & 24 & $7.78 \pm 0.17$ & $7.29 \pm 0.09(6)$ & $8.54 \pm 0.25(31)$ & $6.95 \pm 0.18$ & $7.18 \pm 0.30(3)$ \\
\hline NGC 2004 & 21700 & 3.50 & 0 & 128 & $7.58 \pm 0.18(1)$ & $6.84 \pm 0.16(2)$ & $8.42 \pm 0.33$ & $6.97 \pm 0.23$ & $7.15 \pm 0.37(3)$ \\
\hline 2004 & 26090 & 3.85 & 2 & 32 & $7.80 \pm 0.19$ & $7.60 \pm 0.12(6)$ & $8.44 \pm 0.11(30)$ & $7.02 \pm 0.19$ & $7.22 \pm 0.27(3)$ \\
\hline NG & 21700 & 3.35 & 4 & 133 & $7.56 \pm 0.18$ & $7.03 \pm$ & $8.38 \pm 0$ & $6.90 \pm 0.22$ & $7.17 \pm 0.35$ \\
\hline $\mathrm{NC}$ & 20450 & 3.30 & 2 & 109 & $7.57 \pm 0.1$ & $7.10 \pm$ & $8.39 \pm 0$ & $7.03 \pm 0.22$ & $7.24 \pm 0.38$ \\
\hline & 700 & 3.40 & 5 & 70 & & & & $6.99=$ & \\
\hline $\mathrm{NC}$ & 500 & 3.60 & 5 & 138 & (1) & (2) & & 6.90 & \\
\hline & 500 & 4.15 & 6 & 7 & 7.9 & & 8.3 & $7.24 \pm$ & (3) \\
\hline $\mathrm{N}$ & 21700 & 3.40 & 1 & 114 & $7.78 \pm 0$ & $6.97 \pm$ & $8.43 \pm$ & $7.19 \pm$ & $7.23 \pm$ \\
\hline 2004 & 21700 & 3.45 & 1 & 91 & $7.82 \pm 0$ & $6.99 \pm$ & $8.43 \pm$ & $7.07 \pm$ & $21 \pm$ \\
\hline 2004 & 21500 & 3.50 & 3 & 134 & $7.62 \pm 0$ & $6.90 \pm$ & $8.53 \pm 0$ & $7.02 \pm$ & $7.24 \pm$ \\
\hline C 2004-061 & 20990 & 3.35 & 1 & 40 & $7.91 \pm 0.22(1)$ & $6.99 \pm 0.11(3)$ & $8.30 \pm 0.24(16)$ & $7.13 \pm 0.25$ & $7.17 \pm 0.33$ \\
\hline NGC 2004-062 & 31750 & 4.40 & 1 & 106 & $7.89 \pm 0.25$ & $<7.33 \pm 0.26(1)$ & $8.04 \pm 0.21(2)$ & $6.83 \pm 0.21(1)$ & $7.21 \pm 0.25$ \\
\hline NGC 2004-063 & 21700 & 3.50 & 0 & 107 & $7.72 \pm 0.23$ & $6.97 \pm 0.21$ & $8.55 \pm 0.35(12)$ & $7.03 \pm 0.27$ & $7.17 \pm 0.38$ \\
\hline NGC 2004-064 & 25900 & 3.70 & 6 & 28 & $7.78 \pm 0.25$ & $7.53 \pm 0.09(5)$ & $8.37 \pm 0.11(29)$ & $7.08 \pm 0.25$ & $7.18 \pm 0.25(3)$ \\
\hline NGC 2004-065 & 20450 & 3.40 & 1 & 223 & $7.70 \pm 0.22(1)$ & $<7.19 \pm 0.29(1)$ & - & $7.34 \pm 0.30$ & $7.15 \pm 0.46(1)$ \\
\hline NGC 2004-066 & 25700 & 3.70 & 2 & 238 & $7.78 \pm 0.26(1)$ & $7.98 \pm 0.25(2)$ & $8.49 \pm 0.16$ & $6.88 \pm 0.25$ & $7.21 \pm 0.39$ \\
\hline NGC 2004-068 & 20450 & 3.65 & 1 & 62 & $7.69 \pm 0.24(1)$ & $7.01 \pm 0.30(1)$ & $8.28 \pm 0.33(7)$ & $7.11 \pm 0.28$ & $7.23 \pm 0.38$ (2) \\
\hline NGC 2004-069 & 28000 & 4.00 & 5 & 178 & $7.90 \pm 0.24$ & $7.84 \pm 0.21(2)$ & $8.19 \pm 0.11(7)$ & $7.17 \pm 0.22(1)$ & $7.19 \pm 0.25$ \\
\hline NGC 2004-070 & 27400 & 3.90 & 4 & 46 & $2(1)$ & $.10(4)$ & & $7.15 \pm 0$ & $7.19 \pm 0.25$ \\
\hline & 22950 & 3.50 & 2 & 98 & $7(1)$ & $7.49 \pm 0$ & $8.59 \pm 0$. & $7.00 \pm$ & (3) \\
\hline & 23000 & 3.65 & 1 & 37 & $7.93 \pm 0.17(1)$ & $7.02 \pm 0.09$ (4) & $8.39 \pm 0.27(29)$ & $7.12 \pm 0.20$ & $7.23 \pm 0.34(3)$ \\
\hline & 27375 & 4.25 & 4 & 130 & & $<7.42 \pm$ & $8.51 \pm 0.14(3)$ & $7.02 \pm 0.14$ & $5(3)$ \\
\hline & 21700 & 3.55 & 2 & 116 & & $7.06 \pm 0$ & $8.34 \pm 0.31$ & $7.07 \pm 0$ & $4(3)$ \\
\hline $2004-076$ & 20450 & 3.65 & 1 & 37 & $7.70 \pm 0.1$ & $6.91 \pm 0$ & $8.45 \pm 0.33(22)$ & $7.01 \pm 0.22$ & $7.24 \pm 0$ \\
\hline 2004-077 & 29500 & 4.00 & 6 & 215 & $7.98 \pm 0.17(1)$ & $7.65 \pm 0$. & $8.47 \pm 0.14$ (5) & $7.10 \pm 0.13$ & $7.24 \pm 0.32$ \\
\hline $2004-078$ & 21700 & 3.55 & 1 & 115 & $7.61 \pm 0.11$ & $6.74 \pm 0.1$ & $8.31 \pm 0.33(7)$ & $6.95 \pm 0.18$ & $7.16 \pm 0.36(3)$ \\
\hline 2004-079 & 21700 & 3.60 & 2 & 165 & $7.65 \pm 0.19$ & $7.60 \pm 0.26(2)$ & $8.50 \pm 0.34(5)$ & $7.03 \pm 0.24(1)$ & $7.15 \pm 0.35(3)$ \\
\hline 2004-080 & 20450 & 3.40 & 0 & 85 & $7.64 \pm 0.29$ & $7.09 \pm 0.29(2)$ & $8.51 \pm 0.36(17)$ & $7.05 \pm 0.34$ & $7.24 \pm 0.40$ \\
\hline NGC 2004-081 & 26800 & 4.00 & 2 & 105 & $7.88 \pm 0.18$ & $7.14 \pm 0.15(2)$ & $8.37 \pm 0.13(16)$ & $7.14 \pm 0.17$ & $7.20 \pm 0.25$ \\
\hline NGC 2004-082 & 25700 & 4.10 & 1 & 161 & $8.03 \pm 0.30$ & $7.47 \pm 0.28$ & $8.28 \pm 0.17(12)$ & $7.41 \pm 0.30$ & $7.16 \pm 0.33(2)$ \\
\hline 2004-084 & 27395 & 4.00 & 3 & 36 & $7.90 \pm 0.20(1)$ & $7.28 \pm 0.14$ (4) & $8.42 \pm 0.12(26)$ & $7.17 \pm 0.20$ & $7.25 \pm 0.24$ \\
\hline NGC 2004-085 & 20450 & 3.40 & 4 & 150 & $7.63 \pm 0.25(1)$ & $7.25 \pm 0.26(2)$ & $8.41 \pm 0.35(11)$ & $7.10 \pm 0.30$ & $7.21 \pm 0.37$ \\
\hline
\end{tabular}


Table 2. continued.

\begin{tabular}{|c|c|c|c|c|c|c|c|c|c|}
\hline Star & $\begin{array}{l}T_{\text {eff }} \\
(\mathrm{K})\end{array}$ & $\begin{array}{l}\log g \\
(\mathrm{dex})\end{array}$ & $\begin{array}{c}\xi_{\mathrm{vt}} \\
\left(\mathrm{km} \mathrm{s}^{-1}\right)\end{array}$ & $\begin{array}{c}v \sin i \\
\left(\mathrm{~km} \mathrm{~s}^{-1}\right)\end{array}$ & $\begin{array}{c}\text { Carbon } \\
\text { (dex) }\end{array}$ & $\begin{array}{l}\text { Nitrogen } \\
(\text { dex })\end{array}$ & $\begin{array}{c}\text { Oxygen } \\
\text { (dex) }\end{array}$ & $\begin{array}{l}\text { Magnesium } \\
\text { (dex) }\end{array}$ & $\begin{array}{c}\text { Silicon } \\
\text { (dex) }\end{array}$ \\
\hline NGC 2004-086 & 21700 & 3.85 & 6 & 14 & $7.65 \pm 0.12(1)$ & $7.21 \pm 0.16(4)$ & $8.68 \pm 0.33(27)$ & $6.78 \pm 0.17(1)$ & $7.17 \pm 0.33(3)$ \\
\hline NGC 2004-087 & 25700 & 4.40 & - & 35 & $7.65 \pm 0.24(1)$ & $6.93 \pm 0.15(2)$ & $7.98 \pm 0.15(24)$ & $6.84 \pm 0.24$ & $6.80 \pm 0.20$ \\
\hline NGC 2004-088 & 20450 & 3.65 & 8 & 200 & $7.65 \pm 0.20(1)$ & $<7.18 \pm 0.26(1)$ & $8.45 \pm 0.35$ & $6.85 \pm 0$ & $7.20 \pm 0.32$ \\
\hline NGC 2004-09 & 32500 & 4.10 & - & 16 & $7.79 \pm 0.22(1)$ & $7.65 \pm 0.13$ & $8.44 \pm 0.12(24)$ & $6.97 \pm$ & $7.15 \pm 0.17$ \\
\hline NGC 2004-09 & 26520 & 4.05 & 0 & 40 & $8.08 \pm 0.24(1)$ & $7.29 \pm 0.09(5)$ & $8.38 \pm 0.12(27)$ & $7.29 \pm 0.25$ & $7.26 \pm 0.27$ \\
\hline NGC 2004-0 & 20450 & 3.40 & - & 84 & $7.04 \pm 0.08$ & $<6.99 \pm 0.19$ & $8.49 \pm 0.36$ & $6.92 \pm 0.19$ & $6.99 \pm 0.37$ \\
\hline NGC 2004-0 & 25700 & 4.10 & - & 138 & $7.71 \pm 0.20(1)$ & $7.13 \pm 0.17(1)$ & $8.22 \pm 0.17(16)$ & $7.23 \pm 0$ & $7.00 \pm$ \\
\hline NGC 2004- & 21700 & 3.60 & 2 & 62 & $7.71 \pm 0.13(1)$ & $7.06 \pm 0.17(2)$ & $8.46 \pm$ & $7.06 \pm$ & $7.21 \pm$ \\
\hline NGC & 21700 & 3.75 & 1 & 90 & $7.44 \pm 0.15$ & $<7.34 \pm 0$ & $8.56 \pm$ & $6.61 \pm$ & $7.17 \pm 0$ \\
\hline NGC & 21700 & 3.40 & 2 & 119 & $7.61 \pm 0.23(1)$ & $7.06 \pm 0.20$ & $8.47 \pm 0$ & $6.95 \pm 0.27$ & $7.16 \pm 0$ \\
\hline NGC 2004-100 & 26800 & 3.70 & 6 & 323 & $7.85 \pm 0.11(1)$ & $<7.29 \pm 0.13$ & $8.24 \pm 0.11$ & - & $7.13 \pm 0.33$ \\
\hline NGC 2004-101 & 21700 & 3.45 & 3 & 131 & $7.65 \pm 0.24(1)$ & $7.59 \pm 0.27(2)$ & $8.44 \pm 0.33(13)$ & $6.96 \pm 0.27$ & $7.16 \pm 0.37$ \\
\hline NGC 2004-103 & 21500 & 3.85 & 1 & 35 & $7.71 \pm 0.32(1)$ & $6.95 \pm 0.28(2)$ & $8.29 \pm 0.33(13)$ & $6.98 \pm 0.36$ & $7.19 \pm 0.34$ \\
\hline NGC 2004-104 & 25700 & 3.90 & 1 & 274 & $7.97 \pm 0.29$ & $<7.28 \pm 0.26(1)$ & $8.08 \pm 0.19$ & $7.36 \pm 0.28$ & $7.23 \pm 0.40$ \\
\hline NGC 2004-105 & 25700 & 3.90 & 2 & 235 & $8.00 \pm 0.24$ & $<7.11 \pm 0.21$ & $8.21 \pm 0.19$ & $7.06 \pm 0.23$ & $7.23 \pm 0.36$ \\
\hline NGC 2004-106 & 21700 & 3.50 & 2 & 41 & $7.79 \pm 0.23$ & $7.07 \pm 0.21$ & $8.47 \pm 0.32(21)$ & $7.10 \pm 0.28$ & $7.17 \pm 0.35$ \\
\hline NGC 2004-107 & 28500 & 3.90 & - & 146 & $7.94 \pm 0.21(1)$ & $<7.12 \pm 0.21(1)$ & $8.41 \pm 0.14$ & $7.12 \pm 0.18$ & $7.04 \pm 0.35$ \\
\hline NGC 2004-108 & 22600 & 4.00 & - & 13 & $7.56 \pm 0.16(1)$ & $6.89 \pm 0.08$ & $8.23 \pm 0.20(28)$ & $6.99 \pm 0.20$ & $6.98 \pm 0.26$ \\
\hline NGC 2004-109 & 20450 & 3.50 & 1 & 41 & $7.76 \pm 0.18$ & $6.85 \pm 0.18$ & $8.36 \pm 0.34(15)$ & $7.15 \pm 0.23$ & $7.20 \pm 0.37$ \\
\hline NGC & 21700 & 3.40 & 3 & 121 & $7.62 \pm 0.22(1)$ & $6.95 \pm 0.18$ & 8.62 & $7.03 \pm 0.26$ & $7.20 \pm 0.38$ \\
\hline NGC 2004-111 & 20450 & 3.30 & 2 & 55 & $7.56 \pm 0.14$ & $7.10 \pm 0.23$ & $8.37 \pm 0.34(13)$ & $6.88 \pm 0.21$ & $7.17 \pm 0.38$ \\
\hline 2004-112 & 21700 & 3.65 & 1 & 72 & $7.57 \pm 0.12(1)$ & $<6.85 \pm 0.16(1)$ & $8.42 \pm 0.34$ & $6.86 \pm 0.18$ & $7.18 \pm 0.35$ \\
\hline 2004 & 20450 & 3.25 & 5 & 307 & $7.56 \pm 0.21$ & $<7.29 \pm 0.28$ & $8.30 \pm 0.40$ & $7.03 \pm 0$ & $7.20 \pm 0.47$ \\
\hline 2004-114 & 21700 & 3.60 & 2 & 59 & $7.61 \pm 0.17(1)$ & $7.08 \pm 0.19$ & $8.28 \pm 0.32(18)$ & $6.99 \pm 0.23$ & $7.18 \pm 0.34$ \\
\hline 2004-116 & 21700 & 3.55 & 3 & 63 & $7.61 \pm 0.17(1)$ & $6.93 \pm 0.16(2)$ & $8.60 \pm 0 . ?$ & $6.85 \pm 0.21$ & $7.23 \pm 0.35$ \\
\hline & 21700 & 3.60 & 4 & 75 & $7.58 \pm 0.14(1)$ & $7.11 \pm 0$. & $8.64 \pm 0 . ?$ & $7.03 \pm 0.20$ & $7.22 \pm 0.35$ \\
\hline NGC 2004-119 & 23210 & 3.75 & - & 15 & $7.78 \pm 0.22(1)$ & $6.95 \pm 0.04$ & $8.35 \pm 0.19(29)$ & $7.17 \pm 0.25$ & $7.14 \pm 0.31$ \\
\hline
\end{tabular}

- The values in brackets indicate the number of lines observed for each species. The atmospheric parameters and rotational velocities are taken from Papers III and IV. Alternative stellar identifiers can be found in Papers I and II. Abundances are presented on the scale 12+log[X/H]. A correction has been applied to the carbon abundances. 